\title{
Timing of interfacial diffusion and (stereo)crystallization to tailor mechanical properties of additively manufactured poly(lactides)
}

Citation for published version (APA):

Srinivas, V. (2020). Timing of interfacial diffusion and (stereo)crystallization to tailor mechanical properties of additively manufactured poly(lactides). [Doctoral Thesis, Maastricht University]. Maastricht University. https://doi.org/10.26481/dis.20201013vs

Document status and date:

Published: 01/01/2020

DOI:

10.26481/dis.20201013vs

Document Version:

Publisher's PDF, also known as Version of record

Please check the document version of this publication:

- A submitted manuscript is the version of the article upon submission and before peer-review. There can be important differences between the submitted version and the official published version of record.

People interested in the research are advised to contact the author for the final version of the publication, or visit the DOI to the publisher's website.

- The final author version and the galley proof are versions of the publication after peer review.

- The final published version features the final layout of the paper including the volume, issue and page numbers.

Link to publication

\footnotetext{
General rights rights.

- You may freely distribute the URL identifying the publication in the public portal. please follow below link for the End User Agreement:

www.umlib.nl/taverne-license

Take down policy

If you believe that this document breaches copyright please contact us at:

repository@maastrichtuniversity.nl

providing details and we will investigate your claim.
}

Copyright and moral rights for the publications made accessible in the public portal are retained by the authors and/or other copyright owners and it is a condition of accessing publications that users recognise and abide by the legal requirements associated with these

- Users may download and print one copy of any publication from the public portal for the purpose of private study or research.

- You may not further distribute the material or use it for any profit-making activity or commercial gain

If the publication is distributed under the terms of Article 25fa of the Dutch Copyright Act, indicated by the "Taverne" license above, 


\title{
Timing of interfacial diffusion and \\ (stereo)crystallization to tailor mechanical properties of additively manufactured poly(lactides)
}

\author{
DISSERTATION \\ To obtain the degree of Doctor at Maastricht University, \\ on the authority of the Rector Magnificus, \\ Prof.dr. Rianne M. Letschert \\ in accordance with the decision of the Board of Deans, \\ to be defended in public \\ on $13^{\text {th }}$ October 2020 , at $16: 00$ hrs.
}

by

Varun Srinivas 

Promoter:

Co-promotors:

Assessment committee:
Prof. dr. S. Rastogi

Dr. J.A.W. Harings

Dr.ir. C.S.J. van Hooy-Corstjens (Zuyd Hogeschool)

Prof.dr. R.M.A. Heeren

Prof.dr.ir. S. van der Zwaag (Delft University of Technology) Dr. K. Bernaerts

Prof.dr. R. Graham (University of Nottingham)

This research was funded by Regieorgaan SIA under the RAAK-PRO scheme.

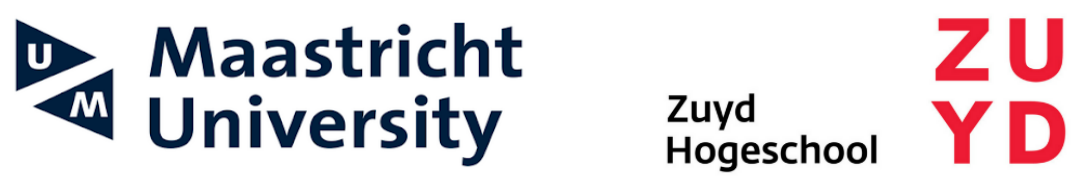


Timing of interfacial diffusion and

(stereo)crystallization to tailor mechanical properties of additively manufactured poly(lactides)

Varun Srinivas 
Timing of interfacial diffusion and (stereo)crystallization to tailor mechanical properties of additively manufactured poly(lactides).

Varun Srinivas, Maastricht University, 2020

ISBN: $978-94-6380-959-7$

Printed by ProefschriftMaken

Cover by Varun Srinivas 



\section{Contents}

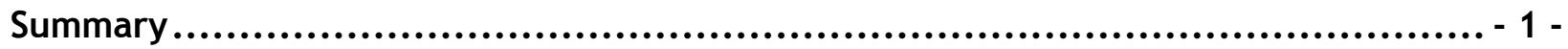

Chapter 1: Introduction ............................................................ - 4 -

Chapter 2: Correlating molecular and crystallization dynamics to macroscopic fusion and thermodynamic stability in fused deposition modeling; a model study on poly(lactides)

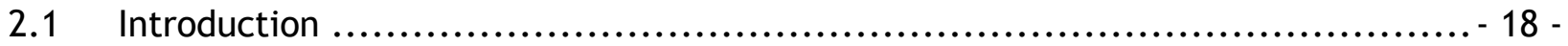

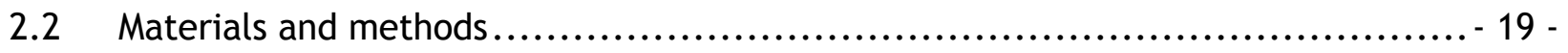

2.3 Results and discussion ..................................................... 21 -

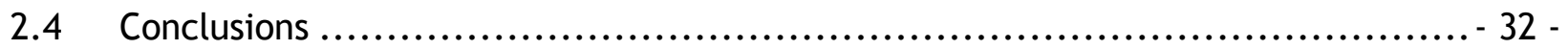

Chapter 3: Promotion of molecular diffusion and/or crystallization in fused deposition modeled poly(lactide) welds

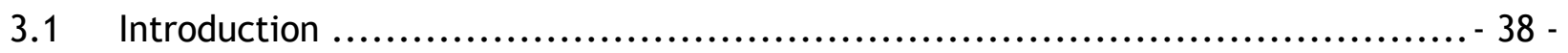

3.2 Materials and methods....................................................... 40 -

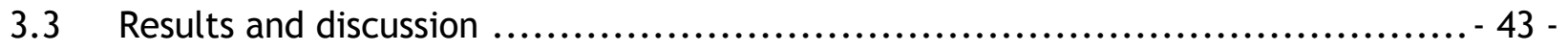

Conclusions ....................................................................... 55 -

Chapter 4: Interfacial stereocomplexation to strengthen fused deposition modeled poly(lactide) welds

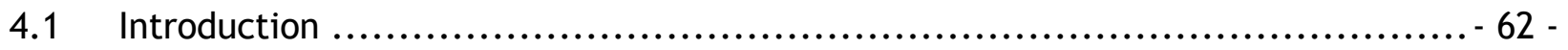

4.2 Materials and methods .......................................................... 63 -

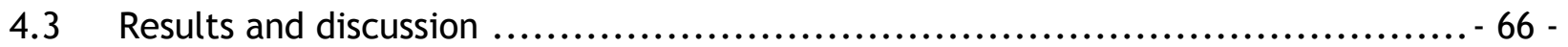

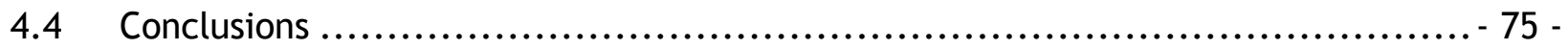

Chapter 5: Time- and length-scales of interfacial stereocomplexation in heterogeneous poly(lactide) melts 
5.2 Materials and methods ........................................................... 84 -

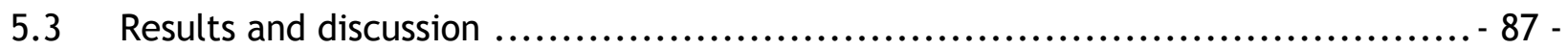

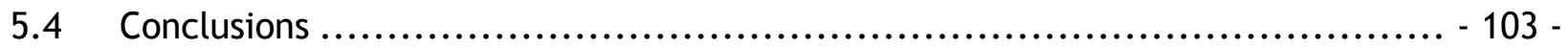

Chapter 6: Valorization; translation of the know-how for the development of products for manufacturing and biomedical industry

6.1 Technological impact in the manufacturing industry ........................... 109 -

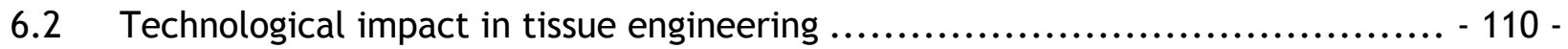

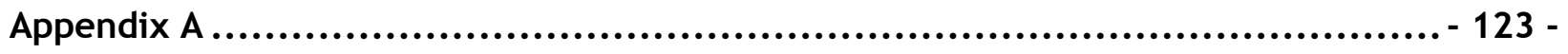

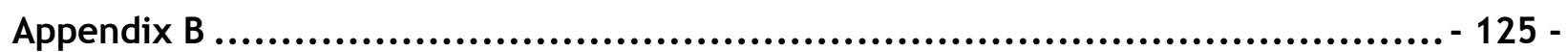

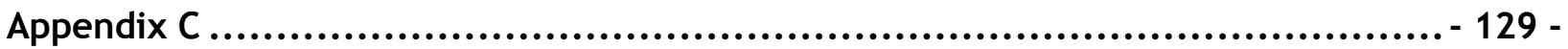

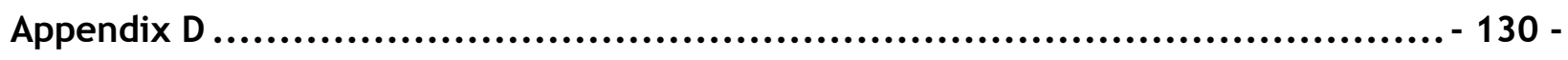






\section{Summary}

Additive manufacturing, more commonly known as 3D printing, is a technology that has developed rapidly as a niche within the field of discrete manufacturing. It provides an avenue to manufacture unique products of high added value that rely on customization and nearly endless design flexibility. We see examples being progressively introduced in several industries like automotive, aerospace, art and medical. Nevertheless, despite successes with materials like metals, ceramics and living materials, a mismatch in product quality and expectations has been restrictive in the mass adoption of thermoplastics in $3 \mathrm{D}$ printing.

Fused deposition modeling (FDM) is by far the most popular 3D printing technology for thermoplastics due to ease of operation, low cost and availability of a variety of materials. It is an extrusion-based technique in which thermoplastics are molten at elevated temperatures and deposited, i.e., welded with high spatial control to form layer-by-layer the desired product. In comparison to other successful construction materials such as metals, polymer molecules are based on long arrays of covalently bound monomeric building blocks. The consequential entanglements between polymer molecules are the origin of the praised mechanical properties accessible via mild melt-based processing conditions, but tremendously reduce the timescales of filament fusion, molecular mixing and crystallization. The aim of this thesis is to generate a fundamental understanding of the physical processes underlying weld formation, assisting in optimum inter-layer mechanical properties in additive manufacturing. Tailoring the molecular design of polymers enables the alignment of timescales of molecular and structural processes with those of this additive manufacturing technology.

To realize stable, durable and high product performance of fused deposition modeled parts, the effect of molecular design parameters and process specific heat fluctuations on structure and morphology is studied in Chapter 2. By selection of poly(lactides) with systematic variations in molar mass and L-enantiomeric purity, molecular and crystallization dynamics are controlled and correlated to (i) print resolution, (ii) mechanically effective bonding via macroscopic fusion and successive molecular diffusion, and (iii) thermodynamic stability. Macroscopic fusion during melt deposition is governed by molecular dynamics of solidification and is positively affected by low print speed and low molar mass. However, low molar mass and high L-enantiomeric purity induce melt crystallization during deposition, limiting interfacial molecular diffusion. By increasing molar mass, crystallization during melt deposition suppresses, facilitating interfacial molecular diffusion that promotes the formation of mechanically effective interfaces. Additionally, structure evolution primarily occurs via cold crystallization in successive annealing 
cycles. Layer-by-layer deposition induces spatial variations in crystallinity and thus thermodynamic instability. These findings highlight that optimum macroscopic mechanics and geometrical stability of fused deposition modeled poly(lactide) parts are compromised and require judicious timing of crystallization and molecular diffusion.

To promote molecular diffusion and/or crystallization at fused deposition modeled weld interfaces, the addition of chemically identical low molar mass fractions to high molar mass PLLA is discussed in Chapter 3. The enantiomeric composition of the low molecular weight fraction is either random (PDLLA) or opposite, promoting molecular diffusion or stereocomplex nucleation respectively. By employing torsional dynamic mechanical analysis, calorimetry and rheology, it is seen that the low molecular weight additives of random $L$ and $D$ enantiomeric composition promote molecular diffusion across the weld interfaces. Nevertheless, the low amount of new entanglements formed upon interfacial mixing and incomplete crystallization induce poor interfacial stiffening and reduced thermodynamic stability. Poly(lactide) stereocomplex enriched interfaces promote crystallization during interfacial mixing, but the hindered diffusion restricted by crystallization at the interface limits molecular mixing and thus the extent of mechanical stiffening. Ultimately, combining melt plasticization and increased crystallization rate by stereocomplex based additives distinctly increases weld stiffness and provides thermodynamic/geometrical stability.

In Chapter 4, a novel route to improve the interlayer weld stiffness is proposed by the timing and spatial direction of poly(lactide) stereocrystallization across fused deposition modeled interfaces. Stereocrystallization in poly(lactides) occurs upon molecular mixing of enantiomerically opposite polymers in the melt state. The resulting stereocrystals possess a melting temperature of approximately 50 to $60^{\circ} \mathrm{C}$ higher than the so-called homocrystals. Per definition, stereocrystals involve two enantiomerically opposite poly(lactide) chains and are hypothesized to physio-mechanically interlock the weld interfaces. Fundamental insight in the kinetics and spatial evolution of interfacial stereocrystallization is studied by rheometry and synchrotron wide angle X-ray diffraction tomography. By alternating deposition of enantiomerically opposite poly(lactides) via a twin nozzle setup, the concept of interfacial stereocrystallization is demonstrated in fused deposition modeling. The printed samples are morphologically and thermomechanically studied by polarized optical microscopy, FTIR microscopy and dynamic mechanical analysis. FTIR microscopy reveals that interfacial diffusion of polymers during fused deposition modeling occurs. The net local heat dosage, which depends on print speed, governs the length-scales of stereocomplexation and thus mechanical reinforcement. Interfacial stereocomplexation of poly(lactides) in FDM leads to a distinct 40\% increase in stiffness and nucleation of bulk filaments, aiding in thermodynamic and geometrical stability. 
Chapter 5 addresses the effect of temperature and relative molar masses in interfacial stereocomplexation of heterogeneous poly(lactide) melts. Rheometry, differential scanning calorimetry and FTIR imaging reveal that a transition from high to low stereocrystallization rate is dictated by a critical network density that impedes diffusion and crystal growth. If the initial relative viscosity is low (affected by temperature and relative molar mass), the time to reach the critical network density is high as a higher number of stereocrystals per chain is demanded in critical network formation. Consequently, the length-scales of stereocrystallization and ultimate mechanical stiffening are high. The relationship reverses in case of a relatively high initial viscosity. The fundamental understanding of the timescales of interfacial diffusion, successive stereocrystallization and nucleation of homocrystallization upon further cooling, assists in the technical realization of mechanically reinforced polymer-polymer interfaces.

In Chapter 6, the future perspectives of this research are explored for the manufacturing and biomedical applications. In the context of biomedical applications, a potential area of future research for tissue engineering is presented whereby spatially directed stiffness in FDM parts is introduced to direct preferential stem cell differentiation in tissue regeneration. 
Chapter 1

\section{Introduction}


"3D printing will change the world" was the title of a Harvard business review back in 2013, which in my opinion aptly captures the hype and enthusiasm surrounding this technology ${ }^{1}$. 3D printing has been identified as "a rare example of a single technology that has become truly disruptive by itself" and as one of the five emerging technology trends that are believed to significantly affect businesses worldwide ${ }^{2}$. When one hears the words "3D printing" or "Additive Manufacturing" (AM), it inherently sounds futuristic. However, the technique is not new and has been around since 1984, when the first stereolithographic 3D printer was produced. For a long time, it was primarily applied for prototyping. Conversely, the last decade has seen the rapid development of numerous 3D printing technologies that have found applications in industries like aerospace, construction, prototyping and biomechanical ${ }^{3-5}$.

3D printing is associated with novel features such as less material wastage, ease to produce complicated geometries, and flexibility in product designs ${ }^{3,5-7}$. This makes $3 \mathrm{D}$ printing not only more flexible than conventional formative molding or casting processes but also economically favourable in scenarios where high financial and time expenditure necessary for the production of molds and tools in formative manufacturing exceeds the usually higher production costs per part in 3D printing ${ }^{2,8}$. In recent years, the overall market situation has been characterized by significant growth in the industry. Revenues from services and products have grown, and worldwide numbers surpassed the value of 5 billion USD in 2015 (as seen in Figure 1.1(a) ${ }^{5,9}$. It is also evident from Figure 1.1(a) that a substantial portion of the growth in the industry has taken place since 2010. This sudden upturn is due to the expiration of the original patents on additive manufacturing, which has given manufacturers the ability to develop new 3D printing devices and technologies. The global 3D printing market was valued at USD 8.08 billion in 2017. With the current growth rate, viz $25.5 \%$ annually, the market is projected to reach USD 49.74 billion by $2025^{6}$. In addition, scientific and technological impact is also steadily increasing, as seen in Figure 1.1(b) which shows the trend tracing the yearly number of scientific publications using the term "additive manufacturing" and "3D printing". Here again, post-2010, a sharp increase in research interest in the field is witnessed. 

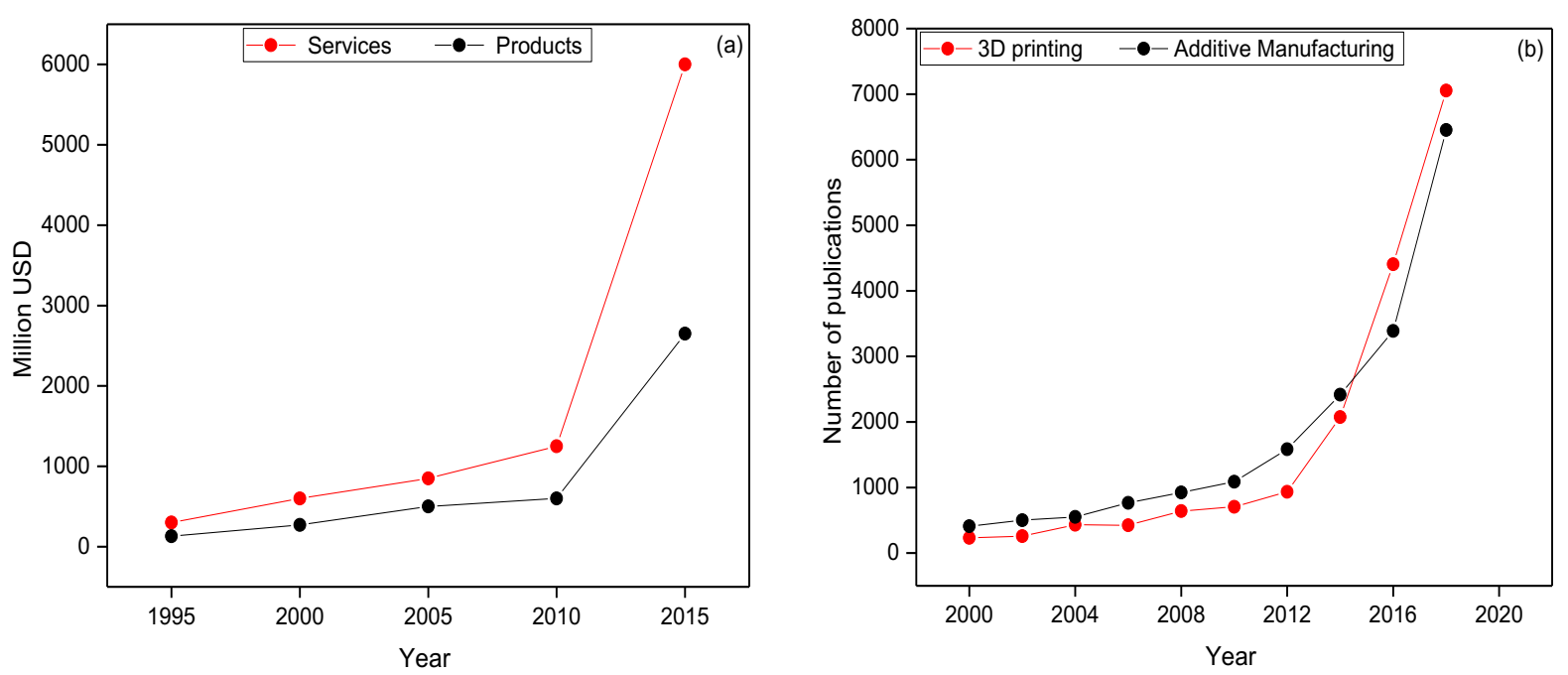

Figure 1.1: (a) Worldwide revenues from 3D printed products and services between 1995 and 2015 ( reproduced from ref 5); (b) The research interest in 3D printing and additive manufacturing, as indicated by the number of publications per annum between 2000 and 2019 (data from Web of Science as of 31/01/2020).

\section{Principal, techniques and materials}

The American Society for Testing and Materials (ASTM) defines additive manufacturing as "the process of joining materials to make objects from 3D model data, layer upon layer, as opposed to subtractive manufacturing methodologies, such as traditional machining" ${ }^{3,5,10}$. It enables the production of customized parts without the need for molds or machining, typical in conventional formative and subtractive fabrication. All 3D printing technologies are characterized by layerby-layer deposition of material distinguished by the principal of material deposition. In 2009, the ASTM International Committee F42 on Additive Manufacturing Technology defined several terms to distinguish and classify additive manufacturing technologies based on their processing principal ${ }^{3-5,7,11}$. In total, six categories were identified. An overview of these categories defined by the production principle, the technologies associated, and materials used are provided in Table 1.1. 
Table 1.1: Categorized 3D printing or additive manufacturing categories with their production principal, associated printing technologies and materials utilized.

\begin{tabular}{|c|c|c|c|}
\hline Category & $\begin{array}{l}\text { Production } \\
\text { principal }\end{array}$ & $\begin{array}{l}\text { 3D printing } \\
\text { technologies }\end{array}$ & Materials \\
\hline Material extrusion & $\begin{array}{l}\text { Material is } \\
\text { selectively } \\
\text { dispensed } \\
\text { through a nozzle }\end{array}$ & $\begin{array}{ll}\text { - } & \text { Fused deposition } \\
\text { - } & \text { 3D dispensing }\end{array}$ & $\begin{array}{l}\text { Polymers: ABS, } \\
\text { Polycarbonate, } \\
\text { Poly(lactide) } \\
\text { (PLA), } \\
\text { Polypropylene } \\
\text { (PP), } \\
\text { Polyetheretherke } \\
\text { tone (PEEK), } \\
\text { Thermoplastic } \\
\text { polyurethanes } \\
\text { (TPU), } \\
\text { Polyamide } \\
\text { Concrete }\end{array}$ \\
\hline Material jetting & $\begin{array}{l}\text { Droplets of } \\
\text { material are } \\
\text { selectively } \\
\text { deposited }\end{array}$ & $\begin{array}{ll}\text { - } & \text { Polyjet printing } \\
\text { - } & \text { Aerosol jet } \\
& \text { printing }\end{array}$ & $\begin{array}{l}\text { Polymers: } \\
\text { Acrylics, } \\
\text { Acrylates, } \\
\text { Epoxides }\end{array}$ \\
\hline Powder fusion & $\begin{array}{l}\text { Thermal energy is } \\
\text { used to fuse } \\
\text { regions of a } \\
\text { powder bed }\end{array}$ & $\begin{array}{ll}\text { - } & \text { Selective laser } \\
& \text { sintering (SLS) } \\
\text { - } & \text { Selective laser } \\
& \text { melting (SLM) } \\
\text { - } & \text { Electron beam } \\
\text { melting }\end{array}$ & $\begin{array}{l}\text { Polymers: } \\
\text { Polyamide, } \\
\text { Polystyrene (PS), } \\
\text { PEEK, TPU, PP }\end{array}$ \\
\hline Sheet Lamination & $\begin{array}{l}\text { Products are } \\
\text { created by } \\
\text { bonding of sheets }\end{array}$ & $\begin{array}{ll}\text { - } & \text { Laminated object } \\
\text { manufacturing }\end{array}$ & $\begin{array}{ll}\text { - } & \text { Metals: } \\
\text { Aluminum alloys, } \\
\text { stainless steel, } \\
\text { Titanium } \\
\end{array}$ \\
\hline $\begin{array}{l}\text { Vat } \\
\text { photopolymerizati } \\
\text { on }\end{array}$ & $\begin{array}{l}\text { Liquid } \\
\text { photopolymer in } \\
\text { a vat is } \\
\text { selectively cured } \\
\text { by light-activated } \\
\text { polymerization }\end{array}$ & $\begin{array}{ll}\text { - } & \text { Multiphoton } \\
\text { polymerization } \\
\text { - } & \text { Direct light } \\
& \text { processing (DLP) } \\
\text { - } & \text { Stereolithography }\end{array}$ & $\begin{array}{l}\text { Polymers: } \\
\text { Acrylics, } \\
\text { Acrylates, } \\
\text { Epoxides }\end{array}$ \\
\hline $\begin{array}{l}\text { Direct energy } \\
\text { deposition }\end{array}$ & $\begin{array}{l}\text { Thermal energy } \\
\text { like laser or } \\
\text { plasma arc are } \\
\text { used to fuse } \\
\text { materials by } \\
\text { melting as they } \\
\text { are being } \\
\text { deposited }\end{array}$ & $\begin{array}{ll} & \text { Direct energy } \\
& \text { deposition (DED) }\end{array}$ & $\begin{array}{l}\text { Metals: } \\
\text { Aluminum alloys, } \\
\text { stainless steel, } \\
\text { Titanium, Silver, } \\
\text { Nickel alloys, } \\
\text { Stainless steel }\end{array}$ \\
\hline
\end{tabular}


Apart from highlighting the broad range of printing technologies available, Table 1.1 further illustrates the plethora of materials used. The variety in material selection facilitates the realization of application-related functionalities and is thus often assigned as a key driver in adoption by several industries. The materials used can be coarsely classified into four classes $3,12,13$ :

- Polymers are considered as the most common materials of use in 3D printing technologies. This class of construction material can be used in the form of filaments in FDM, as powders in the powder-bed methods like SLS, or as resins in stereolithography. Thermoplastic polymers such as acrylonitrile-butadiene-styrene copolymers (ABS), polyamide (PA), polycarbonate (PC) and poly(lactide) (PLA) ${ }^{3,12}$, and thermoset powders like polystyrene, polyamides and photopolymer resins, are the most common types of polymers for 3D printing based on vat polymerization $3,5,12$.

- Metals are mainly employed in the form of powders. Here, SLS, SLM and DED are the main methods of 3D printing that are all based on the fusion of powders by melting or sintering using a laser or electron beam ${ }^{14-16}$.

- Ceramics have gained popularity as they allow the tailored design of materials with a high strength to weight ratio ${ }^{3}$. Ceramics are mainly 3D printed in the form of powders or ink. Powders are sintered using a laser, or fused via an auxiliary adhesive ${ }^{17-19}$. On the other hand, ink-jet printing is used to print suspensions of ceramic particles followed by post-treatment 20,21 .

- Concrete is the most used man-made construction material, which is exploited in construction and infrastructure projects worldwide. The intake of 3D printing by the construction industry has been slow, but evident advantages such as mass-customization, no required formwork and automation exist. Extrusion is the main method for the $3 \mathrm{D}$ printing of concrete $22-24$.

\section{Challenges for 3D printing}

Although 3D printing technologies have made significant strides over the past 25 years, mass adoption is still distant. Technical challenges relating to materials, processes, and applications remain. Although each technology poses specific challenges, some challenges are fundamental to all technologies. Firstly, despite 3D printing processes facilitate much faster product development by reducing the time necessary for design validations and enabling the production of products/prototypes already at early stages in development, the build time of products is slow in comparison to mass production technologies such as injection molding ${ }^{25-27}$. Another major concern is the spatial resolution along the build direction. Insufficient resolution can dramatically influence the quality and functionality of an object ${ }^{3,5,28}$. Due to the layer-by-layer approach of manufacturing, dimensional inaccuracy is inherently expected. To achieve a smooth - 8 - 
surface finish, post-processing techniques like coating, solvent treatment, sanding, or milling are often required. A lot of efforts have been invested in the improvement of the spatial resolution of 3D printing parts. Lastly, as 3D printing enters the area of manufacturing of parts, the functionality of these parts is expected to match or surpass the performance of products fabricated using subtractive and formative technologies. Despite numerous research activities, products produced by 3D printing are often inferior with respect to mechanical properties achieved in formative or subtractive manufacturing ${ }^{12,29-32}$. Depending on the specific process employed, this mechanical inferiority is often material and/or process dependent. Additionally, due to the layered production process, the mechanical properties of parts tend to be anisotropic and the layer interfaces being weak regions face potential residual stresses ${ }^{3,29,33}$.

It is worth noting that build speed and spatial resolution are highly process dependent. On the contrary, the mechanical properties of printed parts are not only influenced by the production process but also by material behaviour. Efforts made earlier to enhance the mechanical properties, especially in the build direction, relied primarily on the optimization of processing parameters. As the consequential improvement in mechanical properties is limited, tailoring material properties is gaining attention, especially for polymers. In the forthcoming section, the specific material and process-related challenges are highlighted for FDM process of thermoplastic polymers and is the primary focus of this thesis.

\section{Process and material specific challenges for FDM}

Amongst the numerous 3D printing technologies, FDM of thermoplastics is the most popular. The popularity is attributed to low-cost equipment, variability in material selection, and easy operation. FDM comprises the 3D extrusion of thermoplastic polymers, which are conventionally mechanically fed as thin filaments from a spool into the extrusion print head. Some variants of the FDM technology like 3D bioplotting and the Arburg plastic freeforming exist and do not need material to be fed as filaments but can be administered as granulate or powder instead ${ }^{34,35}$. During processing, the extruder is heated to a polymer specific process temperature. The temperature is above the melting temperature for semi-crystalline polymers or well above the glass temperature for amorphous polymers. The extruded material is deposited on a substrate, and the geometry is frozen by the solidification of the extruded material. Subsequent layer-bylayer deposition of material results in part buildup.

Despite the popularity and evident advantages, FDM possesses a series of drawbacks. Parts suffer from the same key challenges as other printing technologies, but the surface finish and mechanical properties are particularly poor. The diameter of deposited molten filaments in conventional FDM printers is typically above 100 micrometers, setting the minimum layer height equally. Layer heights in FDM are higher than those encountered in other printing technologies 
like SLS, DLP and SLS ${ }^{36,37}$. Additionally, in comparison to conventional formative melt processing techniques like injection molding, inferior mechanical properties, especially in the build direction, are observed $29,31,38-43$. The origin of the poor mechanical performance originates from the long nature of macromolecules, in which large numbers of monomeric units are covalently connected and their behaviour during the fluctuating processing conditions of FDM.

The macroscopic mechanical integrity depends on the mechanical functionality of the formed interlayer welds at the interface of freshly deposited and previously deposited layers. In the context of FDM, this relates to the degree of entangling of polymer chains at and across the interface ${ }^{44-4729,31}$. The entangling of polymer chains is influenced by numerous factors. Firstly, if in FDM parts molecular diffusion across interfaces of adjacently positioned filaments is inadequate, macroscopic failure under mechanical stress will be governed by failing interfaces that lack randomization and entangling of polymer chains. The origin of this problem is associated with the high molar mass and the entangled nature of polymers that restrict translational molecular motion. Secondly, during processing, more local conformational rearrangements can occur that for example lead to crystallization. Crystallization is preferred from a thermo-mechanical perspective, but under FDM conditions can induce new problems like residual stresses and volumetric contraction that in turn entails geometrical distortions referred to as warping (Chapter 2) ${ }^{48-50}$. Lastly, it is important to note that molecular diffusion, entangling and consequential bonding of the interfaces are thermally driven and are governed by processing parameters like the temperature of the molten extrudate, ambient air conditions, speed of deposition, printing strategy, etc. ${ }^{51-54}$.

To summarize, the weld formation in FDM is a complex process that is dictated by macromolecular dynamics and to facilitate mass adoption as reliable production technology necessitates an in-depth study of molecular diffusion and crystallization under FDM specific timescales of heat administration and dissipation. As previously stated, studies to improve weld mechanics by the optimization of processing conditions have been pursued with marginal effects 51-54. Material related modifications have been mainly focused on the addition of extrinsic reinforcing agents that in most cases influence the intrinsic but not inter-layer weld mechanics. Common examples of extrinsic reinforcement agents include short fibers ${ }^{55,56}$, long fibers ${ }^{57,58}$ and nanoparticles ${ }^{59-61}$.

\section{Aim of this thesis}

The aim of this thesis is to generate a fundamental understanding of the physical processes underlying weld formation in FDM at molecular length-scales assisting in the realization of optimum inter-layer mechanical properties. For this, the primary molecular design of polymeric materials needs to be tailored based on the alignment of timescales of molecular dynamics, the -10 - 
resulting structure evolution and FDM specific processing conditions. Based on the generated knowledge, the thesis assists in the development of novel strategies to enhance inter-layer interfacial weld mechanics by controlling the degree of chain entanglement and /or (co)crystallization at weld interfaces without the addition of extrinsic reinforcing agents.

\section{Outline of this thesis}

- In Chapter 2, the effect of molecular design parameters and FDM specific heat fluctuations on the consequential structure and morphology evolution is studied by selecting poly(lactide) as a model polymer.

- In Chapter 3, tailoring of molecular diffusion and /or crystallization at weld interfaces is studied by the selective addition of low molar mass fractions and nucleating stereocomplex crystals of poly(lactides).

- In Chapter 4, the improvement in interfacial weld stiffness is studied by the timing and spatially controlling poly(lactide) stereocrystallization as mechanical anchors across FDM interfaces.

- In Chapter 5, the mechanism, kinetics and timescales of interfacial poly(lactide) stereocomplexation introduced in Chapter 4 are studied for heterogenous melts consisting of distinct PLLA and PDLA domains by systematic variations in (a) relative molar mass of the mixing PLLA-PDLA phases and (b) crystallization temperature. Furthermore, the concept of interfacial stereocomplexation is explored for other melt-based 3D printing techniques like Selective Laser Sintering (SLS).

- In Chapter 6, the valorization addendum of this thesis is presented. Here, future perspectives augmenting from this research are explored in the context of the industrial manufacturing of mechanically integral and functional parts. Additionally, based on the findings in Chapter 2, a method to produce spatially directed stiffness gradients in FDM parts is introduced that raises expectations in the field of regenerative tissue engineering by surface stiffness directed stem cell differentiation. 


\section{REFERENCES}

(1) Bogue, R., 3D Printing: The Dawn of a New Era in Manufacturing?. Assembly Automation. 2013, 33 (4), 307-311.

(2) Petrick, I. J.; Simpson, T. W., 3D Printing Disrupts Manufacturing. Research Technology Management. November 2013, 12-16.

(3) Ngo, T. D.; Kashani, A.; Imbalzano, G.; Nguyen, K. T. Q.; Hui, D., Additive Manufacturing (3D Printing): A Review of Materials, Methods, Applications and Challenges. Composites: Part B Engineering. 2018, 143, 172-196.

(4) Attaran, M., The Rise of 3-D Printing: The Advantages of Additive Manufacturing over Traditional Manufacturing. Busines. Horizons. 2017, 60 (5), 677-688.

(5) Ligon, S. C.; Liska, R.; Stampfl, J.; Gurr, M.; Mülhaupt, R., Polymers for 3D Printing and Customized Additive Manufacturing. Chemical Reviews. 2017, 117 (15), 10212-10290.

(6) Gardan, J., Additive Manufacturing Technologies: State of the Art and Trends. International Journal of Producation Research. 2016, 54 (10), 3118-3132.

(7) Gebhardt, A., Rapid Prototyping. 2003, I-XV, ISBN 978-1-56990-582-1.

(8) Pereira, T.; Kennedy, J. V.; Potgieter, J. A, Comparison of Traditional Manufacturing vs Additive Manufacturing, the Best Method for the Job. Procedia Manufacturing. 2019, 30, 11-18.

(9) Wohlers, T. T.; Campbell, I.; Diegel, O.; Kowen, J., Wohlers Report 2018: 3D Printing and Additive Manufacturing State of the Industry: Annual Worldwide Progress Report.

(10) Wendel, B.; Rietzel, D.; Kühnlein, F.; Feulner, R.; Hülder, G.; Schmachtenberg, E., Additive Processing of Polymers. Macromolecular Materials and Engineering. 2008, 293 (10), 799-809.

(11) Bourell, D. L.; Rosen, D. W.; Leu, M. C. The Roadmap for Additive Manufacturing and Its Impact. 3D Printing and Additive Manufacturing. 2014, 1 (1), 6-9.

(12) Singh, S.; Ramakrishna, S.; Singh, R., Material Issues in Additive Manufacturing: A Review. Journal of Manufacturing Processes. 2017, 185-200.

(13) Lee, J. Y.; An, J.; Chua, C. K., Fundamentals and Applications of 3D Printing for Novel Materials. Applied Materials Today. 2017, 120-133.

(14) Duda, T.; Raghavan, L. V. ,3D Metal Printing Technology. IFAC-PapersOnLine. 2016, 49 (29), 103109.

(15) Martin, J. H.; Yahata, B. D.; Hundley, J. M.; Mayer, J. A.; Schaedler, T. A.; Pollock, T. M., 3D Printing of High-Strength Aluminium Alloys. Nature, 2017, 549 (7672), 365-369.

(16) Gibson, M. A.; Mykulowycz, N. M.; Shim, J.; Fontana, R.; Schmitt, P.; Roberts, A.; Ketkaew, J.; Shao, L.; Chen, W.; Bordeenithikasem, P., 3D Printing Metals like Thermoplastics: Fused Filament Fabrication of Metallic Glasses. Materials Today. 2018, 21 (7), 697-702.

(17) Sigmund, W. M.; Bell, N. S.; Bergström, L., Novel Powder-Processing Methods for Advanced Ceramics. Journal of American Ceramic Society, 2004, 83 (7), 1557-1574.

(18) Brunello, G.; Sivolella, S.; Meneghello, R.; Ferroni, L.; Gardin, C.; Piattelli, A.; Zavan, B.; Bressan, E., Powder-Based 3D Printing for Bone Tissue Engineering. Biotechnology Advances. 2016, 740753.

(19) Vorndran, E.; Klarner, M.; Klammert, U.; Grover, L. M.; Patel, S.; Barralet, J. E.; Gbureck, U., 3D Powder Printing of B-Tricalcium Phosphate Ceramics Using Different Strategies. Advanced Engeering Materials. 2008, 10 (12), B67-B71.

(20) Derby, B. Inkjet Printing Ceramics: From Drops to Solid. Journal of European Ceramic Society. 2011, 31 (14), 2543-2550.

(21) Moon, J.; Grau, J. E.; Knezevic, V.; Cima, M. J.; Sachs, E. M., Ink-Jet Printing of Binders for Ceramic Components. Journal of American Ceramic Society. 2004, 85 (4), 755-762.

(22) Frank, B.; Wolfs, S.; Zeeshan, A.; Theo, S., Concrete in Construction: Potentials and Challenges of 3D Concrete Printing. Virtual Physical Prototypyping. 2016, 11 (3), 209-225.

(23) Gosselin, C.; Duballet, R.; Roux, P.; Gaudillière, N.; Dirrenberger, J.; Morel, P., Large-Scale 3D Printing of Ultra-High Performance Concrete - a New Processing Route for Architects and Builders. Materials and Design. 2016, 100, 102-109.

(24) Buswell, R. A.; Leal de Silva, W. R.; Jones, S. Z.; Dirrenberger, J., 3D Printing Using Concrete Extrusion: A Roadmap for Research. Cement and Concrete Research. 2018, 37-49.

(25) Stansbury, J. W.; Idacavage, M. J., 3D Printing with Polymers: Challenges among Expanding Options and Opportunities. In Dental Materials. 2016; 32, 54-64.

(26) Conner, B. P.; Manogharan, G. P.; Martof, A. N.; Rodomsky, L. M.; Rodomsky, C. M.; Jordan, D. C.; Limperos, J. W., Making Sense of 3-D Printing: Creating a Map of Additive Manufacturing Products and Services. Additive Manufacturing. 2014, 1, 64-76.

(27) Hofmann, M. 3D Printing Gets a Boost and Opportunities with Polymer Materials. ACS Macro Letters. 2014, 3 (4), 382-386. 
(28) Agarwala, M. K.; Jamalabad, V. R.; Langrana, N. A.; Safari, A.; Whalen, P. J.; Danforth, S. C., Structural Quality of Parts Processed by Fused Deposition. Rapid Prototyping Journal. 1996, 2 (4), 4-19.

(29) Srinivas, V.; van Hooy-Corstjens, C. S. J.; Harings, J. A. W., Correlating Molecular and Crystallization Dynamics to Macroscopic Fusion and Thermodynamic Stability in Fused Deposition Modeling; a Model Study on Polylactides. Polymer. 2018, 142, 348-355.

(30) Levenhagen, N. P.; Dadmun, M. D., Interlayer Diffusion of Surface Segregating Additives to Improve the Isotropy of Fused Deposition Modeling Products. Polymer, 2018, 152, 35-41. .

(31) Srinivas, V.; van Hooy-Corstjens, C. S. J.; Vaughan, G. B. M.; van Leeuwen, B.; Rastogi, S.; Harings, J. A. W., Interfacial Stereocomplexation To Strengthen Fused Deposition Modeled Poly(Lactide) Welds. ACS Applied Polymer Materials. 2019, 1 (8), 2131-2139.

(32) Yin, J.; Lu, C.; Fu, J.; Huang, Y.; Zheng, Y., Interfacial Bonding during Multi-Material Fused Deposition Modeling (FDM) Process Due to Inter-Molecular Diffusion. Materials and Design. 2018, 150, 104-112.

(33) Ahn, S.; Montero, M.; Odell, D.; Roundy, S.; Wright, P. K., Anisotropic Material Properties of Fused Deposition Modeling ABS. Rapid Prototypping Journal. 2002, 8 (4), 248-257.

(34) Neff, M.; Pawelczyk, L., Resilient Hard-Soft Combinations with Plastic Freeforming. ATZproduction Worldwide. 2019, 6 (2), 36-39.

(35) Maher, P. S.; Keatch, R. P.; Donnelly, K.; MacKay, R. E.; Paxton, J. Z., Construction of 3D Biological Matrices Using Rapid Prototyping Technology. Rapid Prototyping Journal. 2009, 15 (3), 204-210.

(36) Ahn, D.; Kweon, J. H.; Kwon, S.; Song, J.; Lee, S., Representation of Surface Roughness in Fused Deposition Modeling. Journal of Materials Processing Technology. 2009, 209 (15-16), 5593-5600.

(37) Agarwala, M. K.; Jamalabad, V. R.; Langrana, N. A.; Safari, A.; Whalen, P. J.; Danforth, S. C., Structural Quality of Parts Processed by Fused Deposition. Rapid Prototyping Journal. 1996, 2 (4), 4-19.

(38) Rodriguez, J. F.; Thomas, J. P.; Renaud, J. E., Mechanical Behavior of Acrylonitrile Butadiene Styrene (ABS) Fused Deposition Materials. Experimental Investigation. Rapid Prototyping Journal. 2001, 7 (3), 148-158.

(39) Song, Y.; Li, Y.; Song, W.; Yee, K.; Lee, K. Y.; Tagarielli, V. L., Measurements of the Mechanical Response of Unidirectional 3D-Printed PLA. Materials and Design. 2017, 123, 154-164.

(40) Ziemian, S.; Okwara, M.; Ziemian, C. W. Tensile and Fatigue Behavior of Layered Acrylonitrile Butadiene Styrene. Rapid Prototyping Journal. 2015, 21 (3), 270-278.

(41) Lee, C. S.; Kim, S. G.; Kim, H. J.; Ahn, S. H., Measurement of Anisotropic Compressive Strength of Rapid Prototyping Parts. Journal of Materials Processing Technology. 2007, 187-188, 627-630.

(42) Wang, J.; Xie, H.; Weng, Z.; Senthil, T.; Wu, L., A Novel Approach to Improve Mechanical Properties of Parts Fabricated by Fused Deposition Modeling. Materials and Design. 2016, 105, 152-159.

(43) Dizon, J. R. C.; Espera, A. H.; Chen, Q.; Advincula, R. C., Mechanical Characterization of 3DPrinted Polymers. Additive Manufacturing. 2018, 20, 44-67.

(44) Schach, R.; Creton, C., Adhesion at Interfaces between Highly Entangled Polymer Melts. Journal of Rheology. 2008, 52 (3), 749-767.

(45) Seppala, J. E.; Hoon Han, S.; Hillgartner, K. E.; Davis, C. S.; Migler, K. B., Weld Formation during Material Extrusion Additive Manufacturing. Soft Matter. 2017, 13 (38).

(46) Mcllroy, C.; Olmsted, P. D. Disentanglement Effects on Welding Behaviour of Polymer Melts during the Fused-Filament-Fabrication Method for Additive Manufacturing. Polymer. 2017, 123, 376-391.

(47) Mcllroy, C.; Olmsted, P. D., Deformation of an Amorphous Polymer during the Fused-FilamentFabrication Method for Additive Manufacturing. Journal of Rheology. 2017, 61 (2), 379-397.

(48) Panda, B. N.; Shankhwar, K.; Garg, A.; Jian, Z., Performance Evaluation of Warping Characteristic of Fused Deposition Modelling Process. International Journal of Advanced Manufacturing Technology. 2017, 88 (5), 1799-1811.

(49) Alsoufi, M.; Abdulrehman, J., Warping Deformation of Desktop 3D Printed Parts Manufactured by Open Source Fused Deposition Modeling (FDM) System. International Journal of Mechanical Mechatronics Engineering. 2017, 17 (4).

(50) Kousiatza, C.; Karalekas, D., In-Situ Monitoring of Strain and Temperature Distributions during Fused Deposition Modeling Process. Materials and Design. 2016, 97, 400-406.

(51) Wendel, B.; Rietzel, D.; Kühnlein, F.; Feulner, R.; Hülder, G.; Schmachtenberg, E., Additive Processing of Polymers. Macromolecular Materials and Engineering. 2008, 799-809.

(52) IV, R. W. G.; Baird, D. G.; Bøhn, J. H., Effects of Processing Conditions on Short TLCP Fiber Reinforced FDM Parts. Rapid Prototyping Journal. 1998, 4 (1), 14-25.

(53) Sun, Q.; Rizvi, G. M.; Bellehumeur, C. T.; Gu, P., Effect of Processing Conditions on the Bonding Quality of FDM Polymer Filaments. Rapid Prototyping Journal. 2008, 14 (2), 72-80. 
(54) Sood, A. K.; Ohdar, R. K.; Mahapatra, S. S., Parametric Appraisal of Mechanical Property of Fused Deposition Modelling Processed Parts. Materials and Design. 2010, 31 (1), 287-295.

(55) Tekinalp, H. L.; Kunc, V.; Velez-Garcia, G. M.; Duty, C. E.; Love, L. J.; Naskar, A. K.; Blue, C. A.; Ozcan, S., Highly Oriented Carbon Fiber-polymer Composites via Additive Manufacturing. Composites Science and Technology. 2014, 105, 144-150.

(56) Zhong, W.; Li, F.; Zhang, Z.; Song, L.; Li, Z., Short Fiber Reinforced Composites for Fused Deposition Modeling. Materials Science and Engineering. A 2001, 301 (2), 125-130.

(57) Yang, C.; Tian, X.; Liu, T.; Cao, Y.; Li, D., 3D Printing for Continuous Fiber Reinforced Thermoplastic Composites: Mechanism and Performance. Rapid Prototyping Journal. 2017, 23 (1), 209-215.

(58) Matsuzaki, R.; Ueda, M.; Namiki, M.; Jeong, T. K.; Asahara, H.; Horiguchi, K.; Nakamura, T.; Todoroki, A.; Hirano, Y. Three-Dimensional Printing of Continuous-Fiber Composites by in-Nozzle Impregnation. Scientific Reports. 2016, 6 (1), 1-7.

(59) Meng, S.; He, H.; Jia, Y.; Yu, P.; Huang, B.; Chen, J., Effect of Nanoparticles on the Mechanical Properties of Acrylonitrile-Butadiene-Styrene Specimens Fabricated by Fused Deposition Modeling. Journal of Applied Polymer Science. 2017, 134 (7).

(60) Sweeney, C. B.; Lackey, B. A.; Pospisil, M. J.; Achee, T. C.; Hicks, V. K.; Moran, A. G.; Teipel, B. R.; Saed, M. A.; Green, M. J., Welding of 3D-Printed Carbon Nanotube-polymer Composites by Locally Induced Microwave Heating. Science Advances. 2017, 3 (6), e1700262.

(61) Marconi, S.; Alaimo, G.; Mauri, V.; Torre, M.; Auricchio, F., Impact of Graphene Reinforcement on Mechanical Properties of PLA 3D Printed Materials. IEEE MTT-S International Microwave Workshop Series on Advanced Materials and Processes for RF and THz Applications, IMWS-AMP 2017, 1, 1- 


\section{Chapter 2}

\section{Correlating molecular and crystallization}

dynamics to macroscopic fusion and thermodynamic stability in fused deposition modeling; a model study on
poly(lactides)

This chapter is based on the following publication:

Srinivas, V.; van Hooy-Corstjens, C. S. J.; Harings, J. A. W., Correlating Molecular and Crystallization Dynamics to Macroscopic Fusion and Thermodynamic Stability in Fused Deposition Modeling; a Model Study on Polylactides. Polymer. 2018,142, 348-355. 


\section{ABSTRACT}

To define molecular parameters for fused deposition modeling of mechanically integral poly(lactide) parts, the effect of intrinsic local heat fluctuations on morphology and structure evolution is studied. Macroscopic fusion during melt deposition is governed by molecular dynamics of solidification and positively affected by low print speed, low molar mass. However, low molar mass and high L-enantiomeric purity induce melt crystallization during deposition, limiting interfacial molecular diffusion. By increasing molar mass, crystallization during melt deposition is suppressed, establishing interfacial molecular diffusion and mechanically effective interfaces. Further structure evolution via cold crystallization is timed in successive annealing cycles. Adding more layers entails a progressive decrease (i) in heat transfer to the build plate and (ii) number of annealing cycles per layer, inducing variations in crystallinity and thus thermodynamic instability. Consequently, macroscopic mechanics and geometrical stability of fused deposition modeled poly(lactides) are compromised by judiciously timed crystallization and process design. 


\subsection{INTRODUCTION}

In FDM, the material is shaped in its melt or liquid state, after which the geometry is preserved upon solidification. The deposited filaments assemble into layers and successively into complete parts while solidifying. During solidification from the melt/cooling, decreased thermal motion is paralleled by increased inter- and intramolecular interactions, leading to a gradual decrease in specific volume and thus contraction and shrinkage on a macroscopic scale ${ }^{1-2}$. If the elementary constituents are sufficiently regular in nature, these thermodynamic processes result in crystallization that is generally accompanied by abrupt and additional volumetric contraction. Thermoplastics differ from other construction materials, such as metals. Covalently connecting the elementary elements into long macromolecules slows down molecular motion and the dynamics of conformational re-arrangements required for macroscopic fusion, molecular diffusion and crystallization.

Crystallization in polymers is often favoured mechanically, especially from a thermomechanical perspective, but its time-scales need to be considered as volumetric contraction, i.e., shrinkage, is largely affected by the kinetics of molecular freezing upon approaching the glass transition temperature, and thus cooling rate ${ }^{2-5}$. If crystallization proceeds beyond the moment of deposition on earlier deposited material, successive shrinkage will lead to immediate macroscopic deformation. This process is referred to as warping and is a common problem observed in FDM ${ }^{6-7}$. If on top of that the polymer is cooled close to or below the glass transition temperature, crystallization may be incomplete, leading to a thermodynamically unstable state. Not reaching its thermodynamically favoured state, means that the lowest specific volume is neither reached, and the printed object is prone to volumetric and thus geometrical changes over longer periods. The phenomenon also exists for non-crystallizable polymers if the formation of the energetically most favourable packing is arrested early ${ }^{8}$. In conventional polymer processing techniques such as injection molding, the differences in specific volume between liquid and crystalline state are minimized by the use of pressure ${ }^{4}$. Since such an option is missing in FDM, thermodynamic instabilities and density fluctuations are often observed in final parts.

The high molar mass of thermoplastics including their entangled nature restricts macroscopic fusion and successive molecular diffusion across interfaces ${ }^{9-11}$. To optimally exploit the intrinsic mechanical response of polymers on a product level, macroscopically imposed stress needs to be transferred to structural and molecular length-scales. If in FDM printed parts molecular diffusion across interfaces of adjacent filaments is inadequate, macroscopic failure under mechanical stress will be governed by failing interfaces that lack randomization and entangling of polymer chains ${ }^{9,12-13}$. Molecular diffusion and eventual bonding are driven by the thermal energy of the extruded material and hence the temperature management of the process. The 
thermal history of interfaces varies throughout the object as the successive deposition of heated material induces initial fast cooling and successive heating 12, 14-16. The complex temperature management is hence of utmost importance to crystallization and bonding and is unique, but scarcely explored.

To ultimately realize stable, durable, and high product performance, we study the effect of molecular design parameters on the timescales of crystallization and molecular dynamics diffusion from a morphological, structural, thermodynamic, and mechanical perspective. Controlled variation in molecular diffusion and crystallization dynamics within the technical time-scales of FDM is perfectly accessible by selecting poly(lactides) (PLA) as its rate of crystallization (i) coincides with the accessible cooling rates ${ }^{17}$, and (ii) can be influenced by enantiomeric purity of the monomers ${ }^{18-20}$; strain rates during flow ${ }^{21-22}$; the addition of nucleating agents ${ }^{23-27}$; and molecular weight. In this work, we focus on enantiomeric purity and molecular weight.

\subsection{MATERIALS AND METHODS}

\subsubsection{Poly(lactides) and printing methodologies}

Three commercially available poly(lactide) grades were provided by Corbion Purac and included two PLAs of 245 and $148 \mathrm{~kg} / \mathrm{mol}$ with L-enantiomeric purity of $99.5 \%$ and one PLA of $245 \mathrm{~kg} / \mathrm{mol}$ with L-enantiomeric purity of $96 \%$. Sample coding is in analogy to the molecular parameters, translating into PLA245_99, PLA148_99, and PLA245_96. Note that the molar masses have been determined by GPC and thus are relative against PMMA standards.

Bars with a uni-directional $0^{\circ}$ deposition strategy and single perimeter were fabricated using an Arburg Freeformer and consisted of 7 layers, being $20 \mathrm{~mm}$ in length, $5 \mathrm{~mm}$ in width, and 2.5 $\mathrm{mm}$ in height as illustrated in Figure 2.1. From a pressurized melt reservoir stringed droplets are deposited through a nozzle with a high frequency piezo actuator. Sample bars were printed under universally optimized conditions that comprise a nozzle temperature of $200{ }^{\circ} \mathrm{C}$ and print speeds of 20 and $50 \mathrm{~mm} / \mathrm{s}$. The ambient (chamber) temperature was set to $70{ }^{\circ} \mathrm{C}$; just above the glass transition temperature of poly(lactides) to promote potential interfacial bonding and sufficiently low to prevent flow or collapse of the entire geometry during printing. 


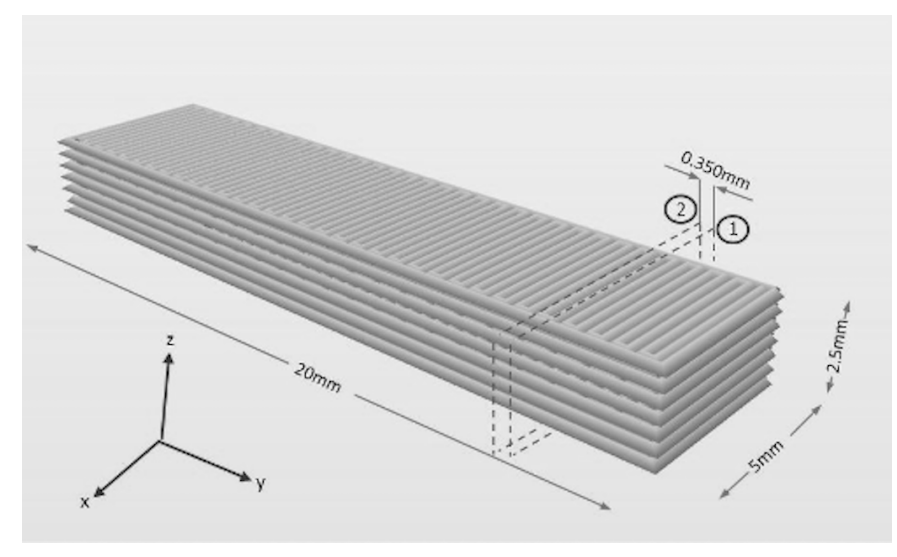

Figure 2.1: Schematic of sample geometry and deposition strategy that is identical in each layer.

\subsubsection{Scanning Electron Microscopy}

Scanning electron microscopy (SEM) was performed on a Philips XL30 system operated at a voltage of $15 \mathrm{kV}$. The samples were taped on a sample holder using conductive carbon tape and a thin gold coating was applied through sputter-coating process.

\subsubsection{Micro-computed X-ray tomography}

The internal morphology of the printed parts was observed by micro-computed tomography (micro-CT) using an X-ray CT scanner (Bruker Skyscan 1272). Micro CT was performed using 40 $\mathrm{kV}, 240 \mu \mathrm{A}$, and $1500 \mathrm{~ms}$ exposure time. Three repetitive images were taken at each slice for averaging and noise reduction. The voxel size was set at $4 \mu \mathrm{m}$ and each image generated had 4032 x 2688 pixels. The images were processed using NRecon reconstruction software and the geometry was constructed after noise reduction. A section of the constructed 3D geometry $(0.35$ mmx3.5mmx2mm), within planes 1 and 2 (see Figure 2.1) was created using CTVox and CTan. The void fraction was calculated for the inlay only after colour transformation, excluding perimeter effects.

\subsubsection{Temperature recording}

Temperature profiles were recorded below the first deposited layer using a $0.15 \mathrm{~mm} \mathrm{~K}$ type thermocouple embedded in the base plate of the printer. The thermocouple was placed in the middle of the bottom layer. High accuracy in data acquisition was guaranteed by a DataQ DI718 data acquisition system read-out system with $1 \mathrm{kHz}$ sampling frequency.

\subsubsection{Structural analysis}

Crystal phase composition and crystallinity of individual layers were determined via 2D wideangle X-ray diffraction using a SAXSLAB's Ganesha instrument equipped with a divergence source producing $X$-ray photons with a wavelength of $1.54 \mathrm{~A}^{\circ}$. The beam center and $\theta$ range were -20 - 
calibrated using the diffraction peaks of silver behenate. Conversion of 2D into 1D data was carried out with Saxsgui V2.13.01 using an automated beam stop mask. Crystallinity was quantified by azimuthal integration of the $2 \mathrm{D}$ diffraction patterns.

\subsubsection{Thermal analysis}

Differential Scanning Calorimetry (DSC) was performed on sections of printed objects using a TA instruments Q 2000 DSC. With $\pm 5 \%$ accuracy, $5 \mathrm{mg}$ of sample was cut and sealed in aluminum pans. Under nitrogen atmosphere, the samples were exposed to a heating cycle ranging from 30 to $200^{\circ} \mathrm{C}$ employing a rate of $10^{\circ} \mathrm{C} / \mathrm{min}$ and an isothermal period of 3 minutes at the temperature limits.

The onset and crystallization half-times for isothermal melt or cold crystallization experiments were supported by fitting Avrami equations for primary crystallization, Figure A1 of Appendix A, and set to 5 and $50 \%$ of the full crystallization time respectively. The crystallinity, $X_{c}$, was calculated by equation 1 where the difference between the melting enthalpy including potential exothermic crystal transitions, $\Delta H_{m}$, and the cold crystallization enthalpy $\Delta H_{c c}$, relative to the enthalpy of a theoretically $100 \%$ crystalline polymer, $\Delta H_{f}{ }^{0}$ being $93 \mathrm{~J} / \mathrm{g}^{28}$.

$$
X_{c}=\frac{\Delta H_{m}-\Delta H_{c c}}{\Delta H_{f}^{0}} \cdot 100
$$

\subsubsection{Dynamic Mechanical characterization}

To test the bonding mechanically, dynamic mechanical testing under torsion was carried out in an Anton Paar rheometer MCR 302. The strain was set at $0.1 \%$, the frequency at $1 \mathrm{~Hz}$ and the storage modulus $\left(G^{\prime}\right)$ was recorded in a temperature range from 40 and $120{ }^{\circ} \mathrm{C}$ and a heating rate of $5{ }^{\circ} \mathrm{C} / \mathrm{min}$.

\subsection{RESULTS AND DISCUSSION}

\subsubsection{Print resolution and macroscopic fusion}

From a technical perspective, fused deposition modeling offers access and freedom in the creation of conventionally non-accessible geometries. However, to realize the resolution described in computer-aided designs, control of the solidification rate is essential. Print resolution and apparent macroscopic fusion were inspected on the exterior by scanning electron microscopy. Figure2.2(a) shows the side view (yz plane) of a PLA148_99 bar printed at a speed of $50 \mathrm{~mm} / \mathrm{s}$. All seven layers, consisting of fused droplet "filaments" are clearly visible. As the build height increases the print resolution decreases progressively, tentatively assigned to excessive flow and thus insufficiently fast solidification. On the contrary, the interface between 
adjacent droplets tends to fade as the layer number increases. Adequate macroscopic fusion and successive bonding via molecular diffusion in and between layers is essential in transferring for example, macroscopic stress to structural and molecular length-scales. The increased degree of apparent macroscopic fusion is clearly witnessed by comparing the xy planes of layer 7 (top) and 1 (bottom) in Figure 2.2(b) and (c). The well-recognized print strategy in Figure 2.2(c) moreover suggests that the high print resolution of layer 1 is at the expense of macroscopic filament fusion.

The influence of molar mass and L-enantiomeric purity on the macroscopic fusion of the bottom xy surfaces at $20 \mathrm{~mm} / \mathrm{s}$ are highlighted in Figure 2.2(d-f). The complete overview is given in Figure $A 2$ of Appendix A. In general, it is observed that the interfaces between adjacent filaments slightly fade at lower print speed. The presence of interfaces in the bottom planes of the PLAs with $99.5 \%$ L-enantiomeric purity (Figures 2.2(d) and (e)) and the absence of distinct interfaces in the bottom layer of the PLA with reduced L-enantiomeric purity (Figure2.2(f)) suggest that fusion in the first layer is less dependent on molar mass, but largely affected by enantiomeric purity. Assessing apparent macroscopic fusion on the exterior of printed bars may be biased by a discrepancy in the set and real layer thickness, smoothing the top surfaces by the nozzle. Hence a study on the internal morphology is demanded.

By means of micro-computed X-ray tomography absorption fluctuations, for example, caused by voids and/or poorly fused interfaces, create phase shifts that can be mapped. Printed bars were analyzed in terms of void fraction, interfaces and homogeneity; all intrinsically correlated to solidification rate and apparent macroscopic fusion, Figure 2.3. The internal void fractions listed in Table 2.1 were calculated by excluding the perimeter effects (Table A1 of Appendix A). At a print speed of $50 \mathrm{~mm} / \mathrm{s}$ a reduction in molar mass from 245 to $148 \mathrm{~kg} / \mathrm{mol}$ induces a reduction in void fraction (from 0.48 to $0.16 \%$ ) and void size as expected from a lower molar mass grade. Above $T_{g}$, time-scales of macroscopic fusion decrease as molar mass decrease ${ }^{29}$. Lowering the L-enantiomeric purity from 99.5 to $96 \%$ results in an even distribution of small voids throughout the volume, despite the overall void fraction remains identical, $0.48 \%$. Upon reducing the print speed to $20 \mathrm{~mm} / \mathrm{s}$ the void fractions are identically affected by the variations in molecular parameters. However, whereas the void fraction of the low molar mass sample remains identical upon lowering the print speed, the void fraction of both poly(lactides) with the identically high molar mass decreases to about $0.31 \%$. This may be explained by the increased time to part completion and thus annealing. The internal morphology analysis evidently demonstrates that low molar mass poly(lactides) promote internal apparent macroscopic fusion. Furthermore, the even distribution of voids across the z-direction discards the smoothing of the top surfaces by compressive forces of the print head. 


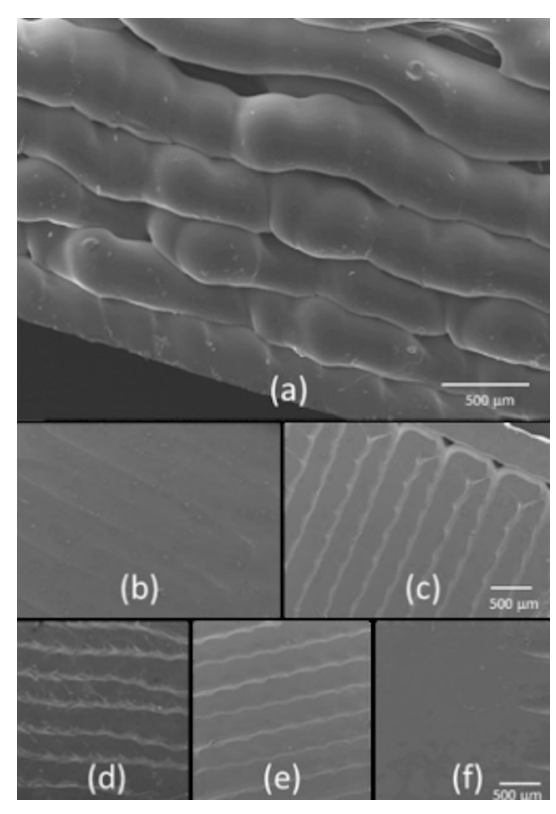

Figure 2.2: Scanning electron micrographs demonstrating solidification, print resolution and fusion of the (a) yz and (b) xy top and (c) xy bottom surfaces of a PLA148_99 printed bar at $50 \mathrm{~mm} / \mathrm{s}$. Figures (df) represent the xy bottom surfaces of PLA245_99, PLA148_99 and PLA245_96 printed at $20 \mathrm{~mm} / \mathrm{s}$.

Table 2. 1: Molecular parameters, void fraction and macroscopic torsional stiffness at $40^{\circ} \mathrm{C}$ for 20 and $50 \mathrm{~mm} / \mathrm{s}$ print speed. The torsional storage modulus was normalized by the void fraction.

\begin{tabular}{|c|c|c|c|c|}
\hline Sample & $\begin{array}{c}\text { Print } \\
\text { speed }\end{array}$ & $\begin{array}{c}\text { Void fraction } \\
{[\%]}\end{array}$ & $\begin{array}{c}\text { Torsional storage modulus [GPa] } \\
\text { as printed G' }\end{array}$ & normalized GN \\
\hline PLA245_99 & 50 & 0.48 & $1.22[ \pm 0.027]$ & 1.24 \\
\hline PLA245_96 & 50 & 0.49 & $0.83[ \pm 0.035]$ & 0.87 \\
\hline PLA148_99 & 50 & 0.16 & $1.05[ \pm 0.035]$ & 1.07 \\
\hline PLA245_99 & 20 & 0.31 & $1.24[ \pm 0.050]$ & 1.29 \\
\hline PLA245_96 & 20 & 0.32 & $0.97[ \pm 0.062]$ & 0.98 \\
\hline PLA148_99 & 20 & 0.15 & $0.98[ \pm 0.028]$ & 1.02 \\
\hline
\end{tabular}

To realize mechanically functional interfaces, macroscopic fusion needs to be complemented by molecular diffusion across interfaces. The macroscopic torsional stiffness at $40^{\circ} \mathrm{C}$, i.e., below the glass transition temperature, was determined by DMTA and is included in Table 2.1. The decreased void fractions and thus apparent macroscopic fusion upon reduced print speed supports a general trend in increased torsional modulus as witnessed for the high molar mass grades and especially PLA245_96 where the storage modulus increases from 0.87 to $0.98 \mathrm{GPa}$. 
Though, a comparison of the absolute values raises a question. Why is the torsional modulus of the low molar mass samples (PLA148) low, despite its increased apparent macroscopic fusion and expected increase in molecular diffusion? The mechanical integrity of the bond depends on the number of chain segments that diffuse across the interface via double random walk, the established interpenetration depth, and state of re-entanglement as modeled by Mcllroy and Olmsted for the amorphous polymer polycarbonate ${ }^{30-31}$. Resolving a one-dimensional heat equation in the $z$-direction demonstrated that the layer-air interface of the first deposited layer cools much faster than the layer-layer interface created by the deposition of any successive layer, partly explaining the poor macroscopic fusion of the bottom layers as detected by SEM. From modeling, it was concluded that although the mutual interpenetration depth, i.e., the bond thickness, is in the order of the polymer size $\left(R_{g}\right)$ its mechanical strength suffers from low entanglement density. In the case of a semi-crystalline polymer like polylactide, crystallization in the bond region may compensate for the lack of entanglements but arrests molecular diffusion at the same time. It is evident that the timing of these temperature-dependent processes demands a more detailed understanding of the dynamic temperature management of FDM as discussed next.

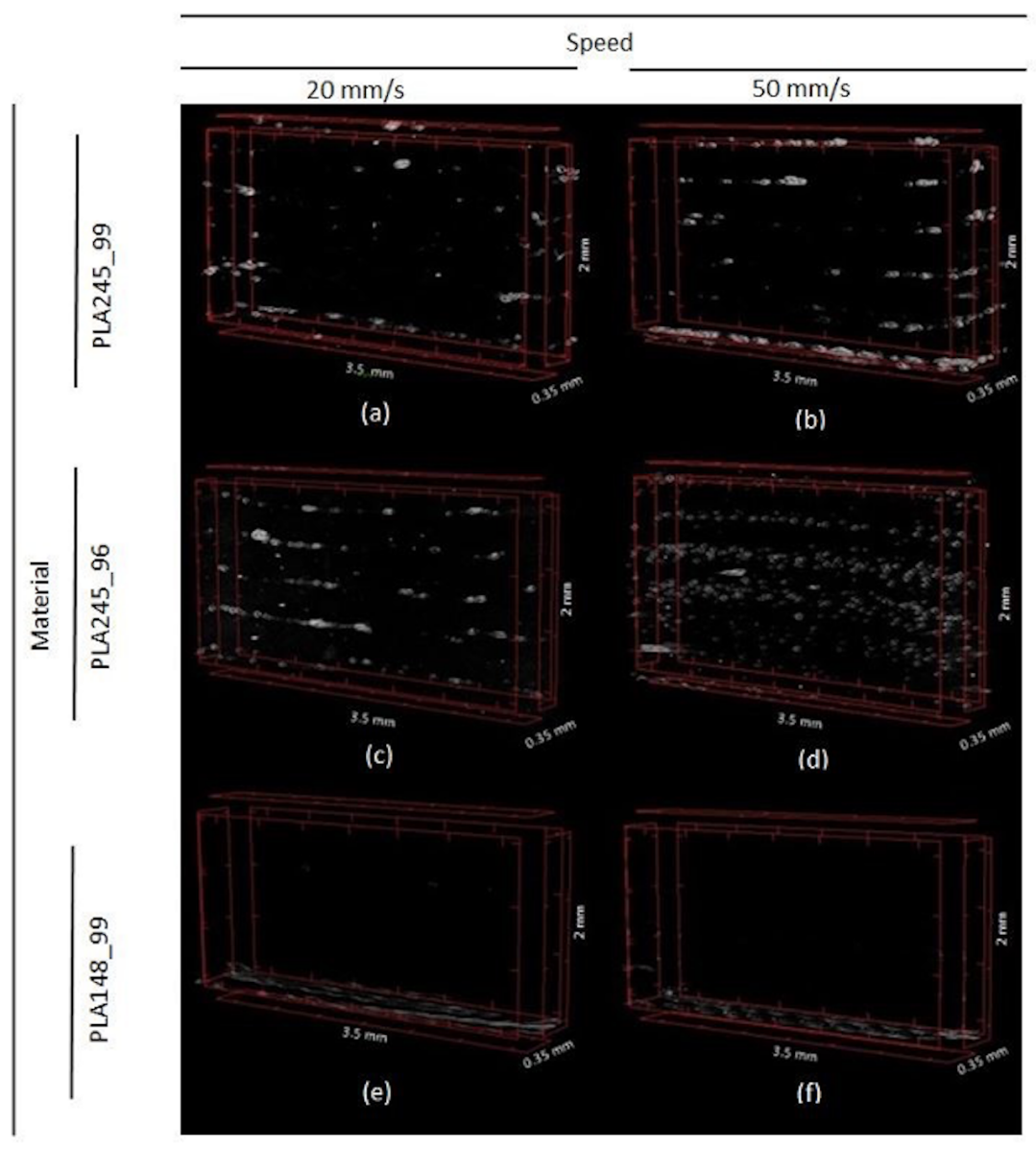

Figure 2.3: Micro-CT reconstructed cross-sectional volumes $(x, z>>y)$ depicting the influence of molar mass, L-enantiomeric purity and print speed on void distribution with $4 \mu \mathrm{m}$ resolution. 


\subsubsection{Heat management and crystallization}

An ultrathin thermocouple was embedded in the build plate to record the temperature profile experienced by the first layer during printing. The temperature profiles recorded while printing seven layers of PLA245_99 at 20 and 50 mm/s are depicted in Figure 2.4(a). Each time the thermocouple passes the nozzle, whether it arises due to depositing a new filament within or on top of a layer, a sharp increase and decrease of temperature is observed. The deposition of a filament in an "additive layer" leads to the highest increase in temperature, but the absolute values of the peak maxima decrease as a function of build height. Moreover, two important trends are observed while comparing the profiles of the different print speeds. Focusing on the peak maxima and minima it is concluded that upon fast printing, i.e., $50 \mathrm{~mm} / \mathrm{s}$, the thermocouple quickly passes the nozzle transferring heat in a relatively short period of time, limiting the maximum temperature rise. Next, due to the high print speed, the thermocouple quickly returns to the nozzle to additively build a new layer, limiting complete heat dissipation as the increase in temperature minima over time suggests. Such a gradual increase in average product temperature promotes excessive flow by effectively reducing the cooling and thus solidification rate. Polymers are good insulators, meaning that the thermal conductivity is low and the heat capacity, especially above $T_{g}$ is high ${ }^{32}$. As both parameters are influenced by the degree of crystallinity, a correlation between thermal heat management and structure evolution is discussed.
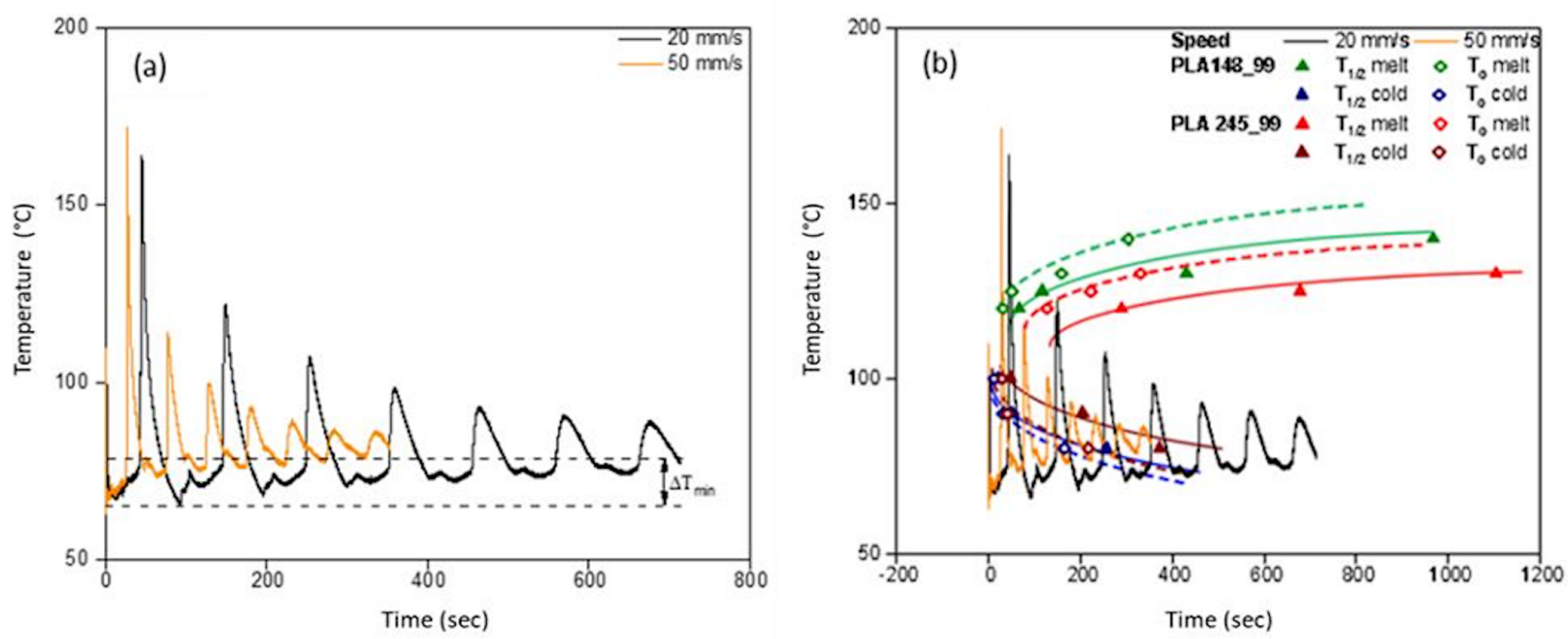

Figure 2.4: (a) Temperature profiles of printing PLA245_99 recorded at 50 and $20 \mathrm{~mm} / \mathrm{s}$ print speed at the center of the bottom layer; (b) overlaid by the isothermal crystallization onset (dotted lines) and half-times (solid lines) of the poly(lactides). Note that under the quiescent conditions, neither melt nor cold crystallization of PLA245_96 occurs within the plotted time-scale. 
The crystallization onset and half-times for the different PLAs as a function of temperature have been overlaid with the temperature profiles in Figure 2.4(b). In general, upon cooling the crystallization rate of polymers initially increases exponentially, governed by the thermodynamic driving force, but gradually arrests as molecular diffusion diminishes upon approaching the glass transition temperature (here $57^{\circ} \mathrm{C}$ ). The trend is conventionally visualized by plotting the crystallization half time as a function of the isothermal crystallization temperature. However, taking the low crystallization rates of poly(lactides) and temperature profiles into account, the crystallization curves in Figure 2.4(b) are composed of melt crystallization (upper; green-red) and cold crystallization upon reheating (lower; brown-blue). It was reported by Saeidlou et al. ${ }^{18}$ that an increase in molar mass and D-enantiomeric content both induce reduced crystallization rates. In Figure 2.4(b), the intersection of the temperature profiles with the crystallization curves suggests that melt crystallization during initial deposition only occurs for the low molar mass grade PLA148_99. Since the crystallization half-time curve (solid green) does not intersect the $50 \mathrm{~mm} / \mathrm{s}$ temperature profile despite its highest crystallization rate, the initial crystallinity of PLA148_99 after deposition is likely to be low. For the higher molar mass grade with equal enantiomeric purity, immediate melt crystallization is further minimized. The deposition of a fresh additive layer is expected to induce cold crystallization, of which the rate is significantly higher. To validate the use of Figure $2.4(\mathrm{~b})$ in understanding the heat management and layer-specific crystallization, and the effect on interand intra-layer bonding via molecular diffusion, detailed structural information such as crystallinity and heat capacity on local length-scales is discussed by layer-specific WAXD and DSC.

To isolate individual layers from a complete three-dimensional sample, the two print units of the printer were optimally exploited. Whereas one unit was filled with the respective PLA, the other unit comprised poly(vinyl-alcohol), PVA, as support material. Since PVA is water soluble and can be printed under identical processing conditions, individual PLA layers can be readily isolated after printing without altering the thermal history, as seen in Figure 2.5. Figure 2.6 shows the two-dimensional WAXD patterns of layers 1, 4 and 7 for a selection of samples. The intensity of the broad amorphous halo relative to the intensity of the crystal induced sharp circumferential diffraction signals directly visualizes the crystalline or amorphous nature of the specific layer. However, the discussion proceeds on the absolute crystallinities, calculated by means of the WAXD and DSC methods described in the materials and methods section. 


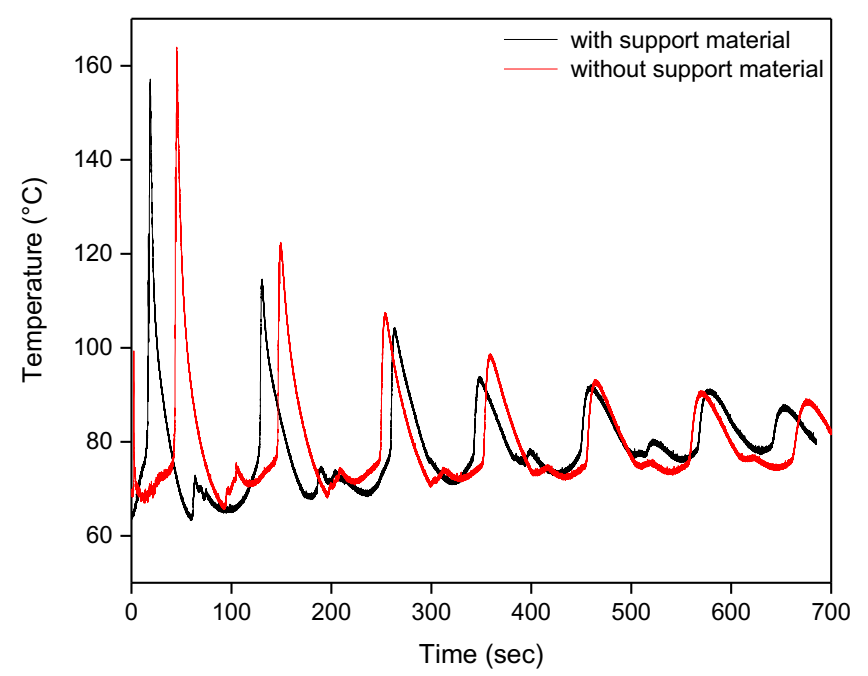

Figure 2.5: Temperature profiles of PLA248_99 printed at $20 \mathrm{~mm} / \mathrm{s}$ printed with (black) and without (red) support material. The exchange of nozzles while switching from PLA to PVA and vice versa, causes a small time shift in the temperature profile.

Despite some variations in absolute crystallinity, the WAXD (Figure 2.6) and DSC (Table 2.2) reveal identical trends. It is evident that within the time-temperature window representing the adopted printing strategy, the poly(lactide)with high enantiomeric purity and low crystallization rate does not crystallize within the time-scales of printing. For the polymer with the highest crystallization rate, PLA148_99, a slow printing, i.e., $20 \mathrm{~mm} / \mathrm{s}$, results in higher crystallinity in all layers. Information on crystallization upon cooling from melt deposition is derived from layers 7 as no successive annealing cycles are imposed on these layers. This causes the crystallinity of the layers 7 to be relatively low with respect to the other layers ${ }^{33-34}$. For example, the crystallinities via WAXD are 18 and $1.3 \%$ for 20 and $50 \mathrm{~mm} / \mathrm{s}$ respectively, coinciding with the tentative interpretation of the intersections in Figure 2.4(b). The intersections of the PLA148_99 melt crystallization curves with the first temperature peak indicate that after deposition crystallization has progressed at $20 \mathrm{~mm} / \mathrm{s}$ and that only a small fraction of crystal entities seems present at $50 \mathrm{~mm} / \mathrm{s}$. The higher increase in the minimum build temperature (induced at high print speed) seems to barely influence the final crystallinity of a freshly deposited layer. So, it is the effective time that the heated nozzle resides at a particular location that promotes crystallinity at low print speed. 


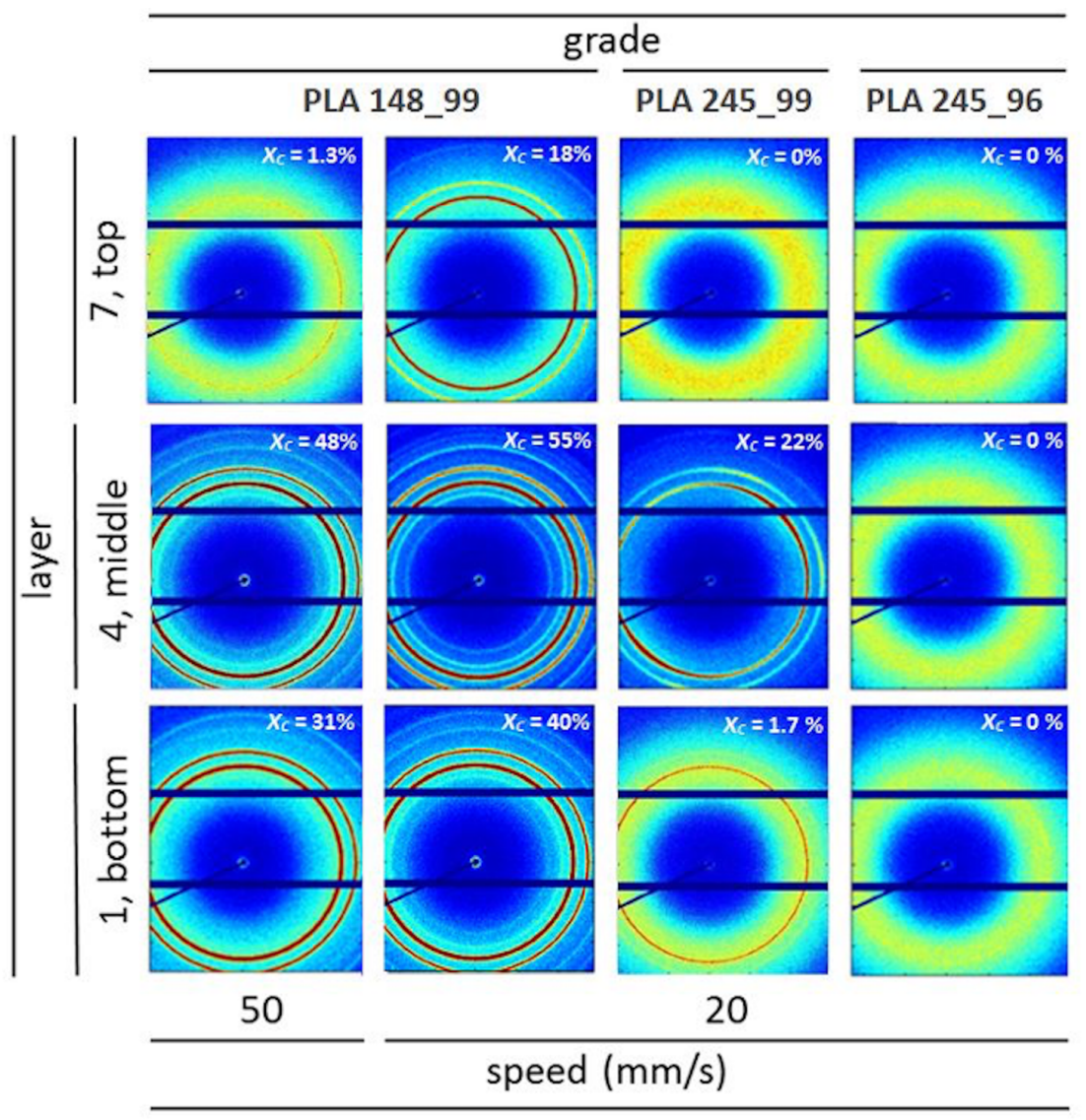

Figure 2.6: 2D WAXD patterns revealing crystallinity (top right numbers) and structural differences between layers induced by print speed, molecular mass and L-enantiomeric purity.

The degree of crystal perfection, indicated by the d-spacing of the 100/200 diffraction signal, depends on the crystallization temperature (Figure 2.7) and is used to provide more information on the moment of crystallization. A low d-spacing, close to the value of the defected $\alpha$ 'phase $0.537 \mathrm{~nm}$, reveals that crystallization occurs below $100^{\circ} \mathrm{C}$ predominantly ${ }^{35-37}$. Crystallization induces an increase in complex viscosity as depicted in Figure $A 3$ of Appendix A, enhancing the solidification rate but limiting molecular diffusion. As the void fraction was the lowest for the low molar mass grades, macroscopic fusion is mainly governed by the intrinsically low melt viscosity prior to crystallization. Despite the crystalline nature and low void fraction, the torsional modulus of low molar mass samples is low in comparison to the less or non-crystalline samples (Table 2.1). Perego et al. ${ }^{38}$ reported that the contribution of crystallinity to the elasticity and flexural modulus outweighs molar mass effects. Thus, even the smallest fraction of crystals hinder molecular diffusion across the filament interfaces, limiting macroscopic stress transfer. The presence of crystals also influences the heat capacity and thermal conductivity of a polymeric material, especially above the glass transition temperature. The change in heat capacity before and after glass transition temperature is given in Table 2.2 to quantify relative changes in heat capacity. Though the crystallinity of layers 7 for PLA148_99 is different, the change in heat capacity $\Delta C_{p}$ barely differs. Note that the difference in crystallinity detected by -28 - 
DSC is smaller than by WAXD. An increase in heat conductivity as expected with increased crystallinity and initially observed at $20 \mathrm{~mm} / \mathrm{s}$, may support thermal conduction and thus a smaller increase in peak minima, Figure 2.4(a). Consequently, the temperature profiles of the three grades with varying crystallization rates are compared in Figure 2.8. It is evident that the temperature minima after cycle 1 and 2 differ and besides scale with the observed crystallinities of the layers 1. The temperature minimum is the lowest for the PLA148_99 that possesses the highest crystallinity, suggesting that crystallinity promotes heat dissipation via conduction to the base plate in the initial layers. As the two profiles after layer 3 match closely the effect of crystallization and consequent changes in heat capacity and thermal conductivity have barely any effect on the observed internal heat management.

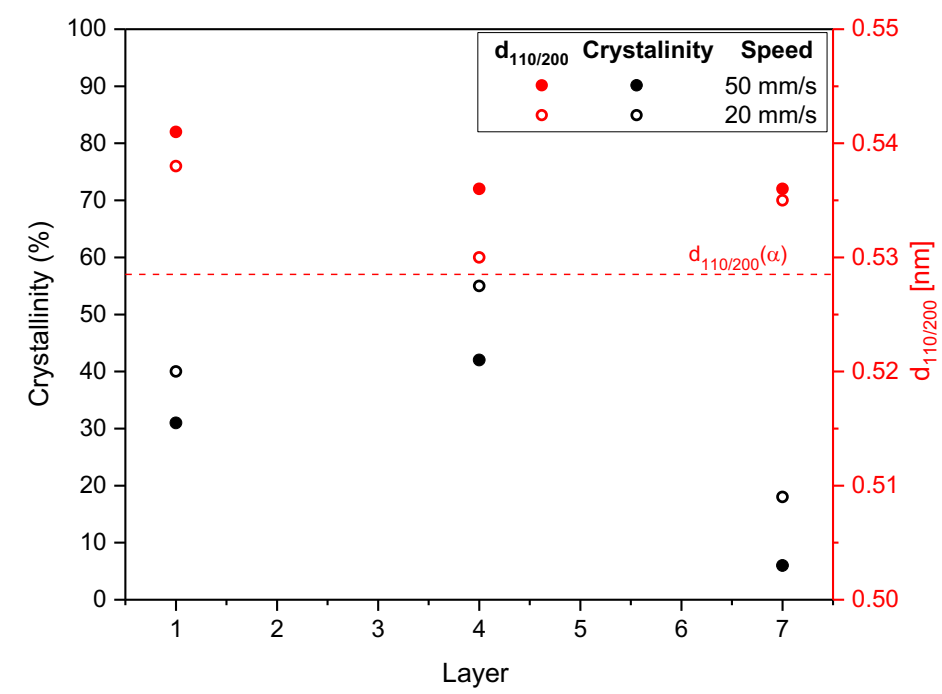

Figure 2.7: Layer specific crystallinity and crystal perfection in the low molar mass grade PLA148_99 printed at 20 and $50 \mathrm{~mm} / \mathrm{s}$ print speed where the $d$-spacing of the 110/200 diffraction signal is indicative for the degree of perfection.

As the printing proceeds by adding new filaments from the melt in successive layers, the previously deposited layers reheat inducing cold crystallization as detected by the high crystallinities of PLA148_99 in layer 1 and 4. The crystallinity is the highest in the middle layers (4) that likely arises due to increased product temperature at the moment of creating the particular layer. Striking is the high degree of crystal perfection of layer 4 created at a speed of $20 \mathrm{~mm} / \mathrm{s}$. The $\mathrm{d}$-spacing is close to the value of the pure $\alpha$ phase, 0.528 . Crystal perfection of the defected packing of the crystalline helical stems by heat (the $\alpha$ ' to $\alpha$ transition ${ }^{35-37}$ ) is exothermic and observed in DSC just before melting, i.e., above $150^{\circ} \mathrm{C}$ (Figure 2.9). It is more likely that the high crystal perfection originates in relatively high crystallization temperatures, typically above $100^{\circ} \mathrm{C}$. Crystallization below $100{ }^{\circ} \mathrm{C}$ induces $\alpha$ ' crystals, while above $120{ }^{\circ} \mathrm{C}$ 
mainly perfect $\alpha$ crystals are observed and between 100 and $120^{\circ} \mathrm{C}$ both crystals phases co-exist 35-36. It is indeed witnessed from the temperature profiles that reheating by depositing a new layer rarely increases the temperature above $100^{\circ} \mathrm{C}$ at $50 \mathrm{~mm} / \mathrm{s}$. At $20 \mathrm{~mm} / \mathrm{s}$, annealing temperatures do reach temperatures above $100^{\circ} \mathrm{C}$ and even $120^{\circ} \mathrm{C}$. Additionally, also the annealing time increases as the nozzle resides for a longer period at a specific location.

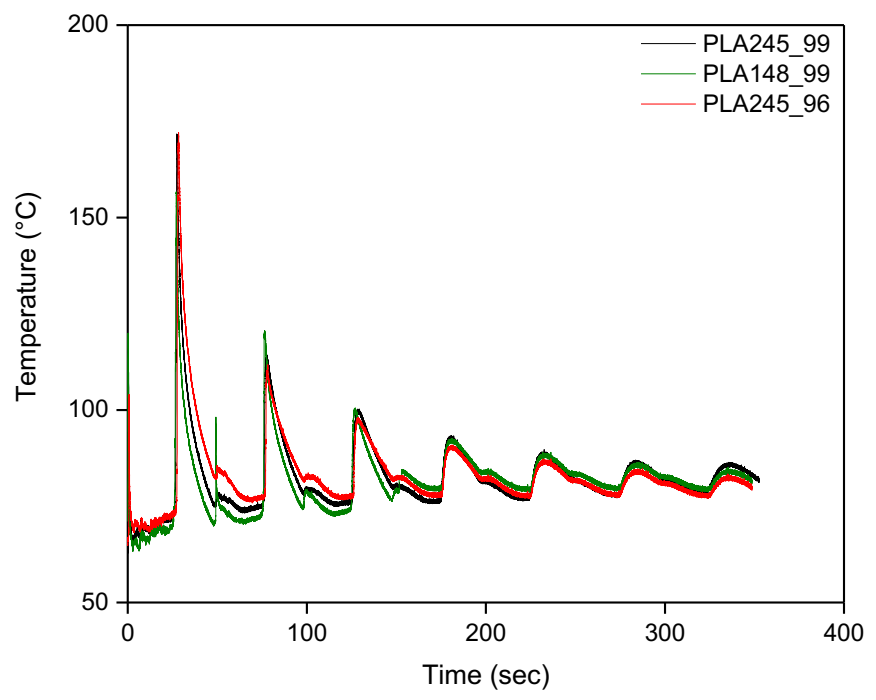

Figure 2.8: Temperature profiles of a PLA245_96, PLA148_99, and PLA245_99 at a print speed $50 \mathrm{~mm} / \mathrm{s}$.
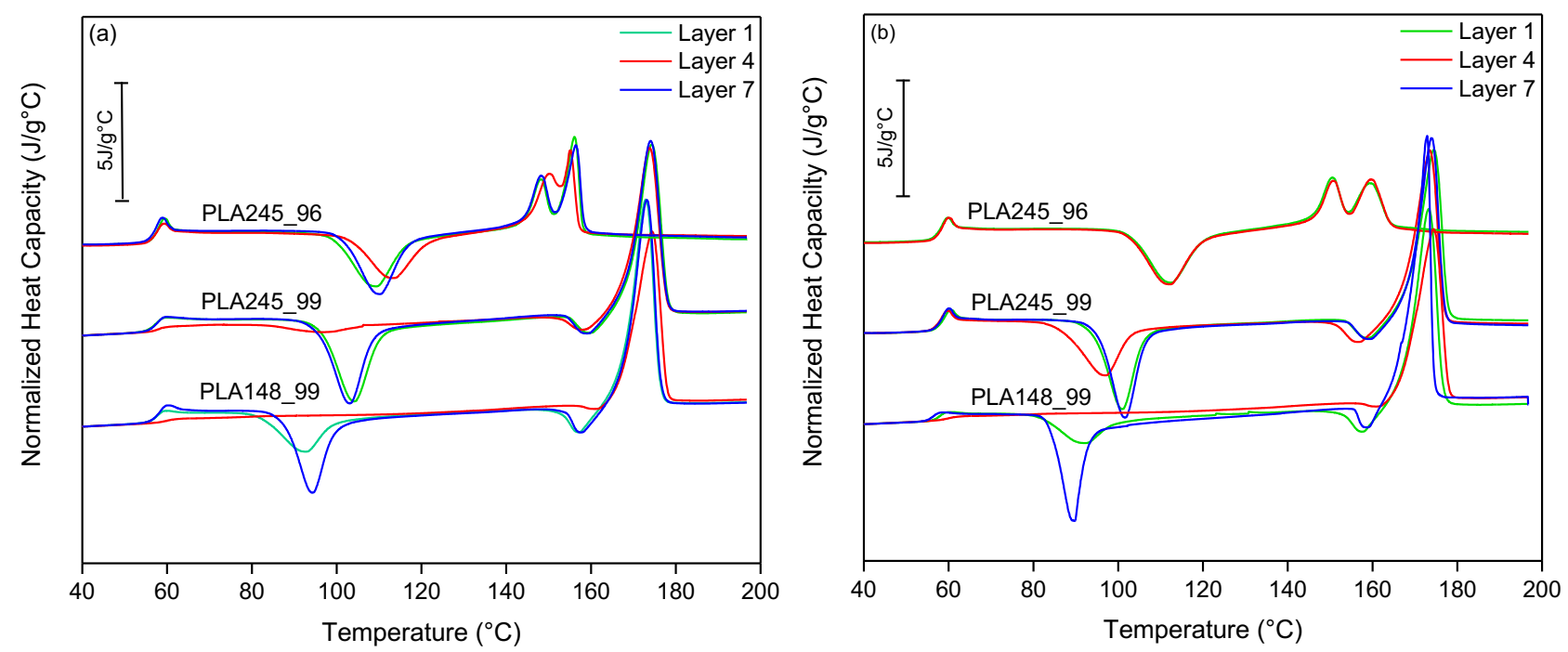

Figure 2.9: Layer specific DSC thermograms revealing spatial variations in crystallinity, heat capacity and thermodynamic stability at (a) 20 and (b) $50 \mathrm{~mm} / \mathrm{s}$. The effect of crystallinity on the heat capacity while printing is apparent from the baseline in the range between the glass transition and cold crystallization and quantified by $\Delta C_{p}$ across the glass transition temperature. 
Upon increasing molar mass and preserving the high enantiomeric purity (PLA245_99), the absolute values of crystallinity decrease due to the inherently low crystallization rate. Despite the additively increasing product temperature, layer 7 is barely or non-crystalline, demonstrating that crystallization, for example, in layer 4 advances via successive annealing cycles. Remarkable for the PLA245_99 is that the crystallinity of the layer 1 remains low despite the successive temperature profile. Due to the massive build plate, the diffusion of heat in layer 1 (below which the thermocouple is placed) is more effective than in successive layers. At the time layer 4 is deposited the increased product temperature and less efficient heat dissipation reduce the cooling rate and increase the effective annealing time and thus the final crystallinity as the temperature peaks. Though the overall crystallinity of the PLA245_99 samples is relatively low, the increased macroscopic torsional storage modulus is the highest. Crystallization starting from the annealing cycles onwards is beneficial to the formation of mechanically more functional interfaces via molecular diffusion before crystallization.

Incomplete crystallization of polymers results in thermodynamic instability. From the DSC (Figure 2.9) and DMTA curves (Figure 2.10) it is evident that cold crystallization occurs unevenly throughout all samples upon heating above the glass transition. Even the samples of poly(lactides) with a reduced L-enantiomeric purity of 96\% (PLA245_96) crystallize eventually, leading to layer-specific volumetric contractions, geometric instability, interfacial stress accumulation and reduced mechanical performance from temperatures close to the glass transition temperature range onwards. Below the glass transition temperature, the timescales and extent of structural rearrangements are however low but inevitable.

Table 2.2: Molecular and process parameters affecting layer specific crystallinity, crystal phase composition and change in heat capacity.

\begin{tabular}{|c|c|c|c|c|c|c|c|c|c|c|}
\hline Sample & $\begin{array}{c}\text { Print } \\
\text { speed } \\
\text { [mm/s ] }\end{array}$ & \multicolumn{2}{|c|}{$\begin{array}{c}\text { Crystallinity } \\
\text { [\%] } \\
\text { WAXD }\end{array}$} & \multicolumn{3}{c|}{$\begin{array}{c}\text { Layer } \\
\text { crystallinity [\%] } \\
\text { DSC }\end{array}$} & \multicolumn{3}{|c|}{$\begin{array}{c}\text { Change in heat } \\
\text { capacity [J/g }\end{array}$} \\
\hline & & $\mathbf{1}$ & $\mathbf{4}$ & $\mathbf{7}$ & $\mathbf{1}$ & $\mathbf{4}$ & $\mathbf{7}$ & $\mathbf{1}$ & $\mathbf{4}$ & $\mathbf{7}$ \\
\hline PLA245_99 & 50 & 0 & 2 & 0 & 6 & 12 & 4 & 0.6 & 0.5 & 0.6 \\
\hline PLA245_96 & 50 & 0 & 0 & 0 & 0 & 0 & 0 & 0.6 & 0.6 & 0.6 \\
\hline PLA148_99 & 50 & 31 & 48 & 1.3 & 32 & 52 & 12 & 0.5 & 0.4 & 0.5 \\
\hline PLA245_99 & 20 & 1.7 & 22 & 0 & 2.5 & 33 & 0 & 0.7 & 0.5 & 0.7 \\
\hline PLA245_96 & 20 & 0 & 0 & 0 & 0 & 0 & 0 & 0.6 & 0.6 & 0.6 \\
\hline PLA148_99 & 20 & 40 & 55 & 18 & 33 & 56 & 20 & 0.6 & 0.4 & 0.6 \\
\hline
\end{tabular}




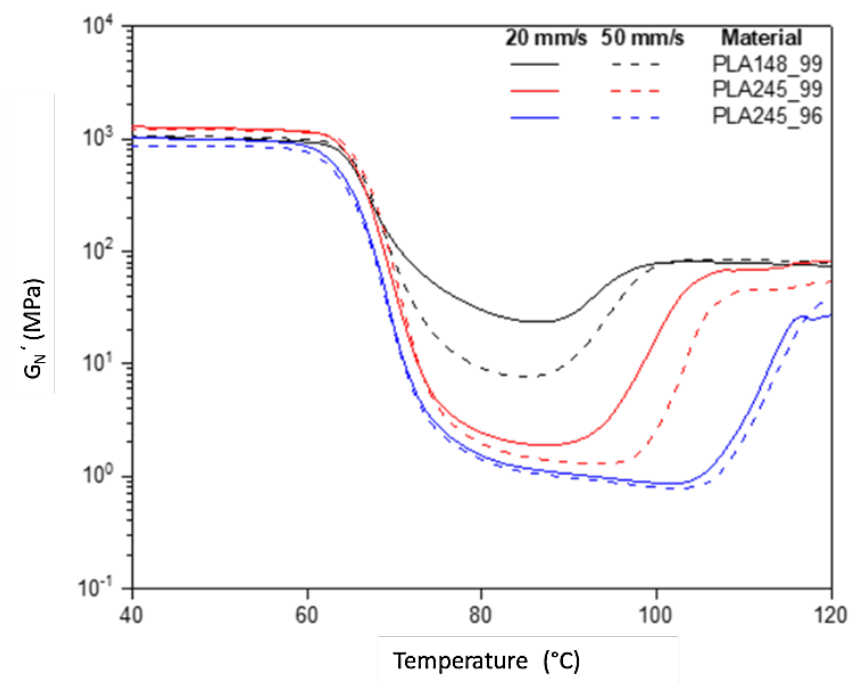

Figure 2.10: Torsional storage moduli $\left(G_{N}\right.$ ') normalized to void fraction as a function temperature. The samples printed at $50 \mathrm{~mm} / \mathrm{s}$ are represented by dashed lines.

\subsection{CONCLUSIONS}

By means of poly(lactides) with systematic variations in molar mass and L-enantiomeric purity, molecular and crystallization dynamics were correlated to (i) print resolution, (ii) mechanically effective bonding via macroscopic fusion and successive molecular diffusion, and (iii) thermodynamic stability. Heat fluctuations intrinsic to fused deposition modeling were recorded at two distinctly different print speeds. Each time a specific xy position traverses in the vicinity of the print head the temperature peaks with respect to the set ambient temperature just above the glass transition. Upon increasing the print speed, the time for effective heat dissipation decreases, increasing the minimum temperature of the object relatively fast. As the average product temperature increases, preservation of print resolution as the number of layers in the z-direction increases is progressively challenged by excessive flow and insufficiently fast solidification. For poly(lactides), macroscopic fusion is defined during melt deposition, promoted by low molar mass and not yet influenced by crystallization. Once the print resolution is established, only the fast crystallizing poly(lactides) with high enantiomeric purity and low molar mass partially crystallize during melt deposition. Upon crystallization, molecular diffusion across inter- and intra-layer interfaces arrests immediately, limiting macroscopic stiffness, albeit the high ultimate crystallinity that arises due to cold crystallization in successive annealing cycles. A reduction in the crystallization rate by increasing molar mass and/or decreasing L-enantiomeric purity suppresses crystallization during melt deposition, rendering time for molecular diffusion and mechanically functional interfaces before cold crystallization in successive annealing sets in. 
By reducing the print speed, the heat flow provided by the print head and polymer melt induce local annealing at relatively high temperatures and long timescales, through which crystallization predominantly progresses. As the number of additively deposited layers increases, the heat dissipation via the build plate decreases due to the high heat capacity and poor thermal conductivity of the polymer. This effect causes temperatures and timescales of annealing to increase in successively added layers, visualizing an increase in the crystallinity of the central layers. However, since the last layers close to part completion are intrinsically exposed to less annealing cycles, the crystallinity lowers progressively. Above the glass transition temperature, cold crystallization induces volumetric changes, geometrical distortion and stress accumulation in all poly(lactides), even for the grade with low L-enantiomeric purity that does not crystallize within the time-scales of printing. Below the glass transition temperature, the structural inhomogeneity and thermodynamic instability make fused deposition modeled PLA parts prone to structural changes, though the timescales and effect on geometrical distortion and interfacial stress accumulation are relatively low. Independent of the application temperature, macroscopic mechanics and geometrical stability of fused deposition modeled poly(lactides) remain to be compromised. Molar mass and L-enantiomeric purity of poly(lactides) aligned with low print speeds are the tools to time crystallization in successive annealing cycles permitting interfacial molecular diffusion. 


\section{REFERENCES}

1. Heydemann, P.; Guicking, H. D., Specific volume of polymers as a function of temperature and pressure. Kolloid-Zeitschrift und Zeitschrift für Polymere. 1963, 193 (1), 16-25.

2. Pethrick, R. A., Book review: Standard pressure-volume-temperature data for polymers. P. Zoller and D. J. Walsh. Technomic Publishing AG, Basel, 1995. ISBN 1-56676-328-2.

3. Zuidema, H.; Peters, G. W. M.; Meijer, H. E. H., Influence of cooling rate on pVT-data of semicrystalline polymers. Journal of Applied Polymer Science. 2001, 82 (5), 1170-1186.

4. Sato, Y.; Inohara, K.; Takishima, S.; Masuoka, H.; Imaizumi, M.; Yamamoto, H.; Takasugi, M., Pressure-volume-temperature behavior of polylactide, poly(butylene succinate), and poly(butylene succinate-co-adipate). Polymer Engineering and Science. 2000, 40 (12), 2602-2609.

5. Brucato, V.; Piccarolo, S.; La Carrubba, V., An experimental methodology to study polymer crystallization under processing conditions. The influence of high cooling rates. Chemical Engineering Science. 2002, 57 (19), 4129-4143.

6. Weng, Z.; Wang, J.; Senthil, T.; Wu, L., Mechanical and thermal properties of $\mathrm{ABS} /$ montmorillonite nanocomposites for fused deposition modeling 3D printing. Materials and Design. 2016, 102, 276-283.

7. Panda, B. N.; Shankhwar, K.; Garg, A.; Jian, Z., Performance evaluation of warping characteristic of fused deposition modelling process. The International Journal of Advanced Manufacturing Technology 2017, 88 (5), 1799-1811.

8. Struik, L. C. E., On the rejuvenation of physically aged polymers by mechanical deformation. Polymer 1997, 38 (16), 4053-4057.

9. Bellehumeur, C.; Li, L.; Sun, Q.; Gu, P., Modeling of Bond Formation Between Polymer Filaments in the Fused Deposition Modeling Process. Journal of Manufacturing Processes. 2004, 6 (2), 170178.

10. Rosenzweig, N.; Narkis, M., Sintering rheology of amorphous polymers. Polymer Engineering and Science. 1981, 21 (17), 1167-1170.

11. Bellehumeur, C. T.; Bisaria, M. K.; Vlachopoulos, J., An experimental study and model assessment of polymer sintering. Polymer Engineering and Science. 1996, 36 (17), 2198-2207.

12. Sun, Q.; Rizvi, G. M.; Bellehumeur, C. T.; Gu, P., Effect of processing conditions on the bonding quality of FDM polymer filaments. Rapid Prototyping Journal. 2008, 14 (2), 72-80.

13. Gurrala, P. K.; Regalla, S. P., Part strength evolution with bonding between filaments in fused deposition modelling. Virtual and Physical Prototyping. 2014, 9 (3), 141-149.

14. Kousiatza, C.; Karalekas, D., In-situ monitoring of strain and temperature distributions during fused deposition modeling process. Materials and Design. 2016, 97, 400-406.

15. Dinwiddie, R. B.; Kunc, V.; Lindal, J. M.; Post, B.; Smith, R. J.; Love, L.; Duty, C. E. In Infrared imaging of the polymer 3D-printing process. Thermosense: Thermal Infrared Applications. 2014, 910502-910512.

16. Yardimci, M. A.; Guceri, S., Conceptual framework for the thermal process modelling of fused deposition. Rapid Prototyping Journal. 1996, 2 (2), 26-30.

17. Di Lorenzo, M. L., Determination of spherulite growth rates of poly(l-lactic acid) using combined isothermal and non-isothermal procedures. Polymer. 2001, 42 (23), 9441-9446.

18. Saeidlou, S.; Huneault, M. A.; Li, H.; Park, C. B., Poly(lactic acid) crystallization. Progress in Polymer Science. 2012, 37 (12), 1657-1677.

19. Garlotta, D., A Literature Review of Poly(Lactic Acid). Journal of Polymers and the Environment. 2001, 9 (2), 63-84.

20. Di Lorenzo, M. L., Crystallization behavior of poly(l-lactic acid). European Polymer Journal. 2005, 41 (3), 569-575.

21. Tang, H.; Chen, J.-B.; Wang, Y.; Xu, J.-Z.; Hsiao, B. S.; Zhong, G.-J.; Li, Z.-M., Shear Flow and Carbon Nanotubes Synergistically Induced Nonisothermal Crystallization of Poly(lactic acid) and Its Application in Injection Molding. Biomacromolecules. 2012, 13 (11), 3858-3867.

22. Steady-shear-induced Isothermal Crystallization of Poly(L-lactide) (PLLA). Journal of Macromolecular Science, Part B. 2008, 47 (3), 511-522.

23. Qiu, Z.; Li, Z., Effect of Orotic Acid on the Crystallization Kinetics and Morphology of Biodegradable Poly(l-lactide) as an Efficient Nucleating Agent. Industrial and Engineering Chemistry Research. 2011, 50 (21), 12299-12303.

24. Li, H.; Huneault, M. A., Effect of nucleation and plasticization on the crystallization of poly(lactic acid). Polymer. 2007, 48 (23), 6855-6866.

25. Kawamoto, N.; Sakai, A.; Horikoshi, T.; Urushihara, T.; Tobita, E., Nucleating agent for poly (Llactic acid)-An optimization of chemical structure of hydrazide compound for advanced nucleation ability. Journal of Applied Polymer Science. 2007, 103 (1), 198-203. 
26. Harris, A. M.; Lee, E. C., Improving mechanical performance of injection molded PLA by controlling crystallinity. Journal of Applied Polymer Science. 2008, 107 (4), 2246-2255.

27. Pei, A.; Zhou, Q.; Berglund, L. A., Functionalized cellulose nanocrystals as biobased nucleation agents in poly(l-lactide) (PLLA) - Crystallization and mechanical property effects. Composites Science and Technology. 2010, 70 (5), 815-821.

28. Fischer, E. W.; Sterzel, H. J.; Wegner, G., Investigation of the structure of solution grown crystals of lactide copolymers by means of chemical reactions. Kolloid-Zeitschrift und Zeitschrift für Polymere. 1973, 251 (11), 980-990.

29. Androsch, R.; Di Lorenzo, M. L.; Schick, C., Effect of molar mass on enthalpy relaxation and crystal nucleation of poly (l-lactic acid). European Polymer Journal. 2017, 96 (Supplement C), 361-369.

30. Mcllroy, C.; Olmsted, P. D., Deformation of an amorphous polymer during the fused-filamentfabrication method for additive manufacturing. Journal of Rheology. 2017, 61 (2), 379-397.

31. Mcllroy, C.; Olmsted, P. D., Disentanglement effects on welding behaviour of polymer melts during the fused-filament-fabrication method for additive manufacturing. Polymer. 2017, 123, 376-391.

32. Pyda, M.; Bopp, R. C.; Wunderlich, B., Heat capacity of poly(lactic acid). The Journal of Chemical Thermodynamics. 2004, 36 (9), 731-742.

33. Drummer, D.; Cifuentes-Cuéllar, S.; Rietzel, D., Suitability of PLA/TCP for fused deposition modeling. Rapid Prototyping Journal. 2012, 18 (6), 500-507.

34. Wang, L.; Gramlich, W. M.; Gardner, D. J., Improving the impact strength of Poly(lactic acid) (PLA) in fused layer modeling (FLM). Polymer. 2017, 114, 242-248.

35. Pan, P.; Zhu, B.; Kai, W.; Dong, T.; Inoue, Y., Polymorphic Transition in Disordered Poly(l-lactide) Crystals Induced by Annealing at Elevated Temperatures. Macromolecules. 2008, 41 (12), 42964304.

36. Yasuniwa, M.; Sakamo, K.; Ono, Y.; Kawahara, W., Melting behavior of poly(l-lactic acid): X-ray and DSC analyses of the melting process. Polymer. 2008, 49 (7), 1943-1951.

37. Wasanasuk, K.; Tashiro, K.; Hanesaka, M.; Ohhara, T.; Kurihara, K.; Kuroki, R.; Tamada, T.; Ozeki, T.; Kanamoto, T., Crystal Structure Analysis of Poly(l-lactic Acid) a Form On the basis of the 2Dimensional Wide-Angle Synchrotron X-ray and Neutron Diffraction Measurements. Macromolecules. 2011, 44 (16), 6441-6452.

38. Perego, G.; Cella, G. D.; Bastioli, C., Effect of molecular weight and crystallinity on poly(lactic acid) mechanical properties. Journal of Applied Polymer Science. 1996, 59 (1), 37-43. 


\section{Chapter 3}

\section{Promotion of molecular diffusion and/or crystallization in fused deposition modeled poly(lactide) welds}

This chapter is based on the following publication:

Srinivas, V.; van Hooy-Corstjens, C. S. J.; Rastogi, S.; Harings, J. A. W., Promotion of molecular diffusion and/or crystallization in fused deposition modeled poly(lactide) welds. Polymer. 2020,202, 348-355, 122637. 


\begin{abstract}
Fused deposition modeled parts of polymeric origin exhibit inferior interlayer mechanical properties. To enhance interfacial weld stiffness, poly(lactide) blends containing low molecular weight polymers of chemically identical but enantiomerically different nature are explored. The enantiomeric composition of the low molecular weight fraction is either random or opposite, promoting molecular diffusion or stereocomplex nucleation respectively. The structureproperty relationship of the interfaces is studied by means of torsional stiffness, calorimetry, and rheology. Fully miscible, non-crystallizable low molecular weight additives of random $L$ and D enantiomeric composition reduce melt viscosity and crystallization rate, cooperatively promoting molecular fusion across the weld interfaces. Nevertheless, the low amount of new entanglements upon interfacial mixing and incomplete crystallization induce poor interfacial stiffening and thermodynamic stability. Poly(lactide) stereocomplex enriched interface promotes crystallization during interfacial mixing. Chains across weld interfaces may be mechanically anchored in crystals, but the hindered diffusion limits molecular mixing and thus the extent of mechanical stiffening. Ultimately, combining melt plasticization and increased crystallization rate by stereocomplex based additives distinctly increases weld stiffness and thermodynamics/geometrical stability of fused deposition modeled poly(lactides).
\end{abstract}




\subsection{INTRODUCTION}

As highlighted in the previous chapters, the mismatch between intrinsic mechanical properties and those achieved via fused deposition modeling, which are expressed in each spatial direction differently, is hindered by limited molecular mixing and entangling at and across weld interfaces ${ }^{1-5}$. The ensemble of covalently bonded long polymer molecules forms a physical network via entanglements, which are the origin of the praised intrinsic mechanical properties that positions polymers uniquely among other construction materials such as metals. However, the long nature of polymer molecules imposes restrictions in welding processes at the macroscopic length scale due to suppressed molecular diffusion that demands cooperative motion triggered by Brownian motion at the segmental scale. Molecular diffusion depends on the number of entanglements per chain, i.e., molar mass, and the chemistry specific conformational motion that severely changes upon (i) shear-induced chain deformation and (ii) rapid cooling of the shaped melt towards the glass transition temperature ${ }^{1}$. However, in the solid-state of a semi-crystalline polymer, the entrapment of polymer chains in multiple crystals is desired from a thermomechanical perspective. However, it hinders molecular diffusion at the interface and induces heterogeneity in density. Retrospectively, misaligned time-scales of the deposition of the adjacent layers during printing, driven by temperature dependent crystallization rates of polymer molecules complexes molecular diffusion between the adjacent layers and is considered to be one of the main causes for the inferior mechanical performance of FDM parts 6,7 .

For example, if the crystallization rate of a polymer is high, like in the case of isotactic polypropylene, molecular diffusion between adjacent layers will be suppressed and will influence chain reptation followed by crystallization at the interface. Moreover, imbalanced time-scales and moments of crystallization at the interface, in the bulk and previously deposited positions will cause shrinkage due to crystallization leading to warpage and evolution of undesired interfacial stresses ${ }^{8}$. On the other hand, if the crystallization rate is considerably low like encompassed in poly(lactides), molecular diffusion at the interface of the adjacent layers is promoted. But incomplete, often layer-specific crystallization results into secondary crystallization and so thermodynamic, mechanical and geometrical instability over time ${ }^{6,9}$. In general, melt shaping and crystallization dynamics depend on (i) process parameters like imposed cooling rates ${ }^{10}$ and relaxation times of the deformed chain influenced by the applied strain rate during flow ${ }^{11}$, (ii) additives like nucleating agents ${ }^{12-14}$, and (iii) molecular weight ${ }^{15}$ and molecular architecture ${ }^{16,17}$.

To overcome the imposed fundamental challenges, researchers have developed strategies to improve the weld interfaces of FDM printed parts. In terms of process parameters, deposition 
strategy ${ }^{18,19}$, filament to filament air gap ${ }^{20}$, layer height ${ }^{21}$, and bed temperature ${ }^{18}$ have been coupled with the mechanical improvement of weld interfaces. Despite considerable efforts, these strategies improve the mechanical properties only to a limited extent. Meanwhile, other researchers studied the problem from the perspective of materials design. Sweeney et al. ${ }^{4}$ developed a concept for welding FDM thermoplastic interfaces using intense localized postheating of carbon nanotubes (CNTs) enriched filament surfaces by microwave irradiation. The promoted local chain mobility, driven by local heating, lead to an improvement in fracture strength up to $275 \%$ in the z-direction (perpendicular to the direction of the layer deposition). Meng et al. ${ }^{22}$ utilized inorganic fillers such as calcium carbonate $\left(\mathrm{CaCO}_{3}\right)$, montmorillonite (MMT), multiwalled carbon nanotubes (MWCNTs), and silica $\left(\mathrm{SiO}_{2}\right)$ in an Acrylonitrile-ButadieneStyrene (ABS) polymer matrix reporting improvement in mechanical strength and reduction in mechanical anisotropy. Recently, Levenhagen and Dadmun showed that the anisotropy of FDM printed PLA parts can be reduced by the addition of low molecular weight additives of chemically identical chains that assist in the formation of entanglements at the filament interfaces ${ }^{23}$. The presence of a low molecular weight (LMW) additive to create a bimodal blend can improve inter-filament adhesion. It was hypothesized that LMW species diffuse more readily across the filament interfaces. The driving force behind this hypothesis is attributed to the ease in relaxation of the low molecular weight constrained chains that (i) tend to migrate to filament surfaces in extrusion imposed flow profiles ${ }^{24,25}$, and (ii) successive low diffusion times between the layers. Subsequently, in a follow-up publication, Levenhagen and Dadmun introduced LMW additives with star type architectures (3-arm and 4-arm) to the bulk material and compared the mechanical properties and structural intra-layer anisotropy ${ }^{26}$. Although these efforts have aided to the current understanding of printed structure-property relationships, the outcome was specific to the geometry of the object and chosen print strategy. To design polymers for FDM, the reversible translation of intrinsic and mechanical properties is only accessible by decoupling the effect of material and processing parameters from geometry.

In this study, we investigate the influence of chemically identical LMW fractions on improving the interlayer weld mechanics of fused deposition modeled PLAs specifically. Besides surface enrichment in LMW entities by flow-induced fractionation, the addition of LMW additives that sufficiently fast relax from the imposed shear rates may be considered beneficial for interlayer chain diffusion. By mapping the heat management during FDM, the influence of material and process parameters can be interpreted independently from shape. PLA has proven to be an ideal model as deficiencies in the alignment of diffusion, crystallization and cooling/heating rates are expressed in fused deposition modeled part mechanics ${ }^{6}$. Besides, by means of the enantiomeric composition of the high, but particularly low molar mass PLAs, molecular diffusion and/or crystallization can be selectively promoted. 


\subsection{MATERIALS AND METHODS}

\subsubsection{Materials}

A commercial poly(L-lactide) (PLLA) grade with weight average molecular weight $(M w) 245$ $\mathrm{kg} / \mathrm{mol}$ and an enantiomeric purity greater than $99 \%$ served as bulk material. To promote molecular diffusion, one series of low molar mass additives is required to be non-crystallizable and well miscible with the bulk material. For these reasons, poly (DL-lactide)s of 25 and 70 $\mathrm{kg} / \mathrm{mol}$ in weight average molecular weight served as diffusion promoting LMW additives. Sample notation is analogous to enantiomeric composition and molar mass, translating to L245, DL25 and DL70. Note that the molar masses were determined by Gel Permeation Chromatography (GPC), relative to Poly(MethylMethAcrylate) (PMMA) 100kg/mol standards in HFIP solvent.

PLA is known to have two enantiomeric forms called poly(L-lactide) (PLLA) and poly(D-Lactide) (PDLA). Upon mixing, an interesting phenomenon occurs. PLLA and PDLA chains co-crystallize, packing as 31 helices in a crystal structure referred to as a stereocomplex (SC) ${ }^{27-29}$. PLA stereocomplex is distinguished from PLLA or PDLA homocrystals by a $50^{\circ} \mathrm{C}$ higher melting point. To promote the crystallization rate of L245 we use the nucleation effect of SC crystals ${ }^{30-32}$. The nucleating effect of the stereocomplex is most efficient when it is formed well before PLLA crystallization upon cooling ${ }^{33}$. Hence, it was decided to prepare SC crystals separately before blending instead of in-situ during blending. SC crystals were prepared by melt mixing and extruding PLLA $(\mathrm{Mw}=140 \mathrm{~kg} / \mathrm{mol})$ and PDLA $(\mathrm{Mw}=100 \mathrm{~kg} / \mathrm{mol})$ in a 50:50 $(\mathrm{w} / \mathrm{w})$ ratio at $240^{\circ} \mathrm{C}$ for 2 minutes. The extruded material contains a high percentage of SC crystals as proven by Differential Scanning Calorimetry (DSC), Figure 3.1. All poly(lactides) were kindly provided by Corbion Purac, The Netherlands. 


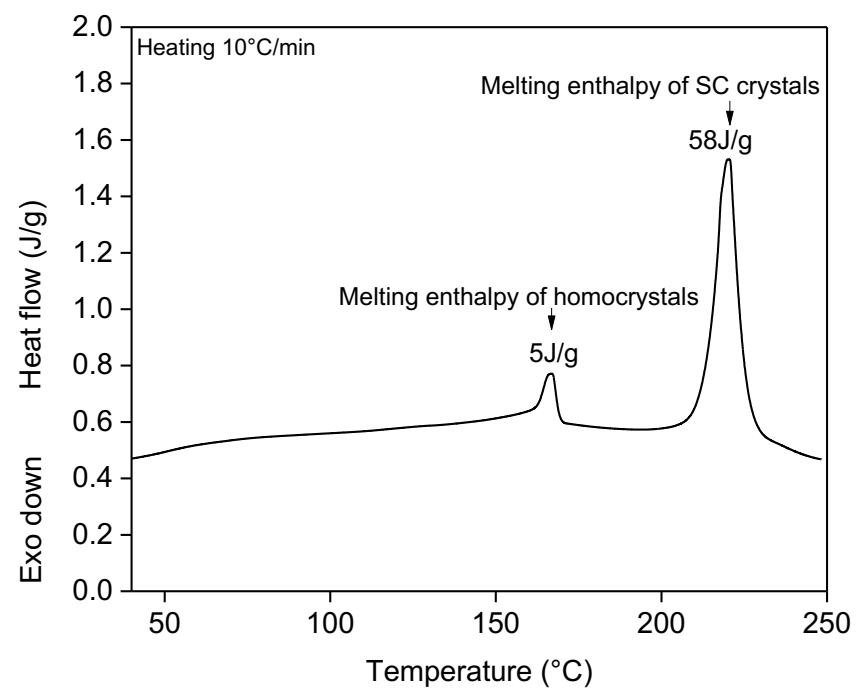

Figure 3.1: DSC thermograms illustrating the formation of SC crystals prepared by melt mixing. The melting enthalpy of homocrystals $(5 \mathrm{~J} / \mathrm{g})$ represents the fraction of PLLA/PDLA chains that do not participate in stereocomplex formation and melting enthalpy of SC crystals $(58 \mathrm{~J} / \mathrm{g})$ indicates the fraction of SC crystals formed. Assuming a value of $146 \mathrm{~J} / \mathrm{g}$ for the melting enthalpy of $100 \%$ crystalline PLA in stereocomplex form, the extruded material contains approximately $40 \%$ SC crystals.

\subsubsection{Preparation of blends}

All materials were dried under vacuum before use. Samples were prepared by mixing different percentages in a DSM Explore twin-screw micro-extruder with a barrel size of $5 \mathrm{ml}$. The samples were processed at $180^{\circ} \mathrm{C}$ for $2 \mathrm{~min}$ and $100 \mathrm{rpm}$ under constant nitrogen flow. Afterwards, the extruded material was shredded into pellets that were subsequently utilized for printing. The blends evaluated in this study can be divided into 4 series. In L245-DL25 and L245-DL70 blends, low molar mass DL25 or DL70 was added in multiple concentrations to the high molar mass L245. L245-SC represents the class of blends prepared in which SC crystals were added to L245. L245DL70-SC blends consist of L245 in combination with both DL70 and SC crystals. The concentration range of the low molar mass additives (DL25 and DL70) and SC crystals was based on the printability at $180^{\circ} \mathrm{C}$, stressing the effects of low molar mass additives while keeping the heat management constant. A printability window was empirically determined based on coupling machine limits to the zero-shear viscosity as evaluated by rheometry measurements.

\subsubsection{Fused deposition modeling of samples}

Upstanding bars with a uni-directional $0^{\circ}$ deposition strategy were fabricated using an Arburg Freeformer. The sample geometry is illustrated in Figure B1 of Appendix B. Via a pressurized melt reservoir, stringed droplets were deposited through a nozzle with a high frequency piezo actuator. All sample bars were printed under optimized printing conditions that comprise a nozzle temperature of $180^{\circ} \mathrm{C}$, an ambient air temperature of $70^{\circ} \mathrm{C}$ and a print speed of $20 \mathrm{~mm} / \mathrm{s}$. An ambient temperature of $70^{\circ} \mathrm{C}$ was chosen as it is above the glass transition temperature 
(thereby promoting interfacial bonding) and sufficiently low to prevent the flow or collapse of the entire geometry during printing.

\subsubsection{Thermal behaviour of blends}

Differential Scanning Calorimetry (DSC) was used to validate ideal mixing and identify changes in crystallization behaviour of the blends. DSC of the blends was performed using a TA instruments Q2000 DSC, weighing $5 \mathrm{mg}$ of material with $\pm 5 \%$ accuracy in hermetically sealed aluminum pans. Samples were heated from 25 to $200^{\circ} \mathrm{C}$ at a rate of $10^{\circ} \mathrm{C} / \mathrm{min}$ and held for 3 minutes isothermally before successively cooling to $25^{\circ} \mathrm{C}$ at a rate of $10^{\circ} \mathrm{C} / \mathrm{min}$. Additionally, isothermal (melt and cold) crystallization experiments were performed to calculate the onset times of crystallization. For melt crystallization, samples were cooled from $200^{\circ} \mathrm{C}$ to the desired isothermal crystallization temperature at a cooling rate of $30^{\circ} \mathrm{C} / \mathrm{min}$ and kept isothermally for 1 hour. For cold crystallization, amorphous samples, which were prepared by melt quenching, were heated to the desired crystallization temperature at $30^{\circ} \mathrm{C} / \mathrm{min}$ and kept isothermally for 1 hour.

\subsubsection{Rheology studies for blends}

Melt rheology of the prepared blends was studied using a TA Instruments DHR1 rheometer operating in parallel plate geometry under nitrogen atmosphere. The plate diameter was $25 \mathrm{~mm}$ and the gap size was set to $0.8 \mathrm{~mm}$. Oscillatory frequency sweep tests were carried out at $180{ }^{\circ} \mathrm{C}$ employing a constant shear strain of $0.5 \%$ and a frequency range of $1-100 \mathrm{rad} / \mathrm{s} .180{ }^{\circ} \mathrm{C}$ was chosen as at this temperature all samples were printable.

\subsubsection{Crystalline morphology of printed samples}

To understand the crystalline morphology, samples were cut into parts with a width of $3 \mathrm{~mm}$ from the top end of the sample. By cutting at the central region of the parts along the $z$-axis with a Leica EM UC7 ultra-microtome, thin slices of $2.5 \mu \mathrm{m}$ thickness were obtained. Polarization Optical Micrographs (POM) of the slices were taken between cross-polarizers using an Olympus BX53 microscope equipped with an Olympus DP26 camera.

\subsubsection{Mapping thermal history of printed samples}

Temperature profiles were recorded below the first deposited layer using a $0.15 \mathrm{~mm} \mathrm{~K}$ type thermocouple embedded in the base plate of the printer. The thermocouple was placed in the middle of the bottom layer. High accuracy in data acquisition was guaranteed by a DataQ DI718 data acquisition system read-out system with $1 \mathrm{kHz}$ sampling frequency. 


\subsubsection{Mechanical Characterization}

To test the bonding mechanically, dynamic mechanical testing under torsion was carried out in an Anton Paar MCR 302 rheometer. The strain was set at $0.1 \%$, the frequency at $1 \mathrm{~Hz}$ and the storage modulus ( $\left.G^{\prime}\right)$ was recorded in a temperature range from $40^{\circ} \mathrm{C}$ to $120^{\circ} \mathrm{C}$ using a heating rate of $5^{\circ} \mathrm{C} / \mathrm{min}$.

\subsection{RESULTS AND DISCUSSION}

\subsubsection{Printability and selection of blends}

To study the effect of the different LMW additives on structure evolution and consequential interlayer weld stiffness, a single melt nozzle temperature is preferred. Using rheometry, the nozzle temperature was empirically linked to the zero-shear viscosity of L245 (Figure B2 of Appendix B) that enabled us to determine a printability window. Any composition possessing a zero-shear viscosity above $5000 \mathrm{~Pa} \cdot \mathrm{s}$ is found to be too viscous for extrusion. Any composition characterized by a zero-shear viscosity below $500 \mathrm{~Pa} \cdot \mathrm{s}$, could not be printed due to uncontrolled fluctuations in volume flow rate and consequential loss in print resolution.
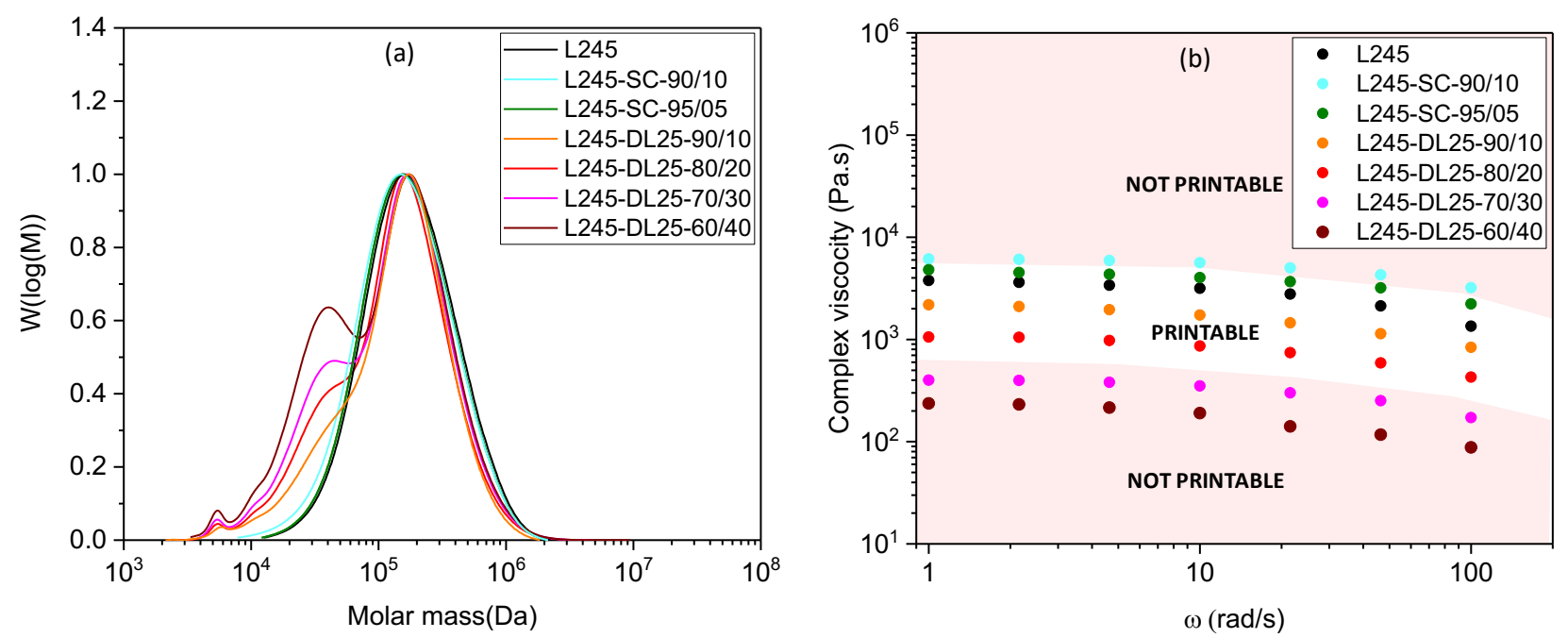

Figure 3.2: Analysis of (a) molar mass distributions and (b) the rheological response of $L 245$ based blends in which different LMW poly(lactides) with variations in enantiomeric composition and molar mass are added. The blends are based on 5 or $10 \mathrm{wt}$-\% stereocomplex (SC) based LMW additives or 10 to $40 \mathrm{wt}-\%$ of non-crystallizable PDLLA of $25 \mathrm{~g} / \mathrm{mol}$ (DL25).

The influence of the LMW additives on the printability of the thus prepared blends, of which the GPC traces are given in Figure 3.2(a), was linked to molecular composition by means of frequency sweep at $180^{\circ} \mathrm{C}$, Figure 3.2(b). Upon increasing the fraction of LMW non-crystallizable additive, clearly expressed by the presence of shoulder at $40 \mathrm{kDa}$ for the L245-DL25 samples in Figure 3.2(a), the complex viscosity decreases. In fact, for LMW DL25 fractions higher than 20 
wt-\% the zero-shear viscosity falls below the lower printability limit that was defined at 500 Pa.s. Since the reduction in viscosity is the highest for the lowest molecular weight DL additive, the highest fraction of LMW DL additive was set at $20 \mathrm{wt}$-\%. Since the melt temperature at the nozzle was fixed at $180^{\circ} \mathrm{C}$, to ensure constant heat management stressing the effect of blend composition on final structure and weld mechanics, the LMW stereocrystals do not melt. As a consequence, the complex and zero-shear viscosity at $180^{\circ} \mathrm{C}$ increases as their weight fraction increases, as observed for the L245-SC samples in Figure 3.2(b). With the addition of $10 \mathrm{wt}$ \% stereocomplex based LMW the viscosity surpasses the upper printability limit of $5000 \mathrm{~Pa} \cdot \mathrm{s}$, restricting the fraction of stereocomplex based LMW additives to 1 and $5 \mathrm{wt}-\%$. The selected blend compositions for the study, of which the GPC traces are given in Figure B3 of Appendix B, are systematically listed and coded in Table 3.1. It is to be noted that for L245-D70-SC blends, the DL70 concentration was set to 19 and $15 \mathrm{wt}$-\% respectively, to have sufficiently high weight fractions of LMW species. To be effective, the stereocomplex-based LMW additives must be dispersed homogeneously throughout the L245 matrix after blending. Polarized optical microscopy of the L245-SC blends heated above the melting temperature of the L245 PLA matrix $\left(200^{\circ} \mathrm{C}\right)$ (see Figure 3.3 ) proofs the homogeneous distribution of micron-sized stereocrystal based domains.

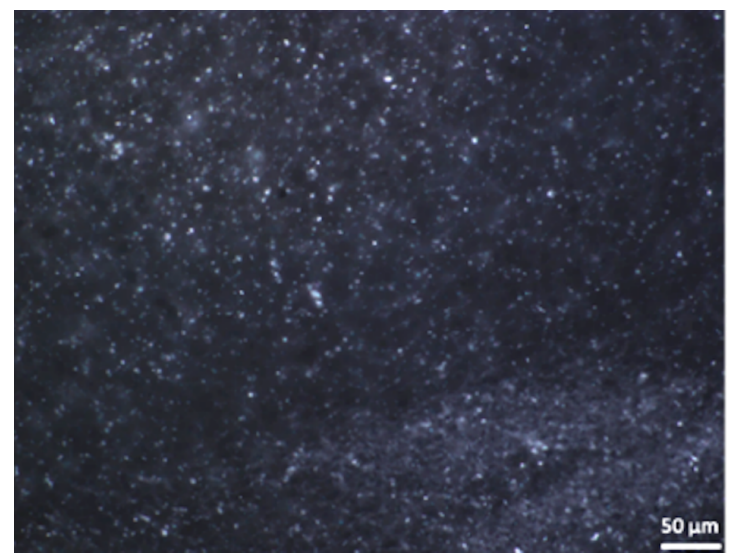

Figure 3.3: Polarized optical micrograph of L245-DL70-SC-15/05 at $200^{\circ} \mathrm{C}$, illustrating the homogenous distribution of micron-sized PLA stereocrystals.

Table 3.1: Composition of the studied blends in wt-\%.

\begin{tabular}{|c|c|c|c|c|c|}
\hline Series & Coding & $\begin{array}{c}\text { L245 } \\
(\mathbf{w t} \%)\end{array}$ & $\begin{array}{c}\text { DL25 } \\
(\mathbf{w t}-\%)\end{array}$ & $\begin{array}{c}\text { DL70 } \\
(\mathbf{w t}-\%)\end{array}$ & $\begin{array}{c}\text { SC (wt- } \\
\%)\end{array}$ \\
\hline \multirow{3}{*}{ L245-DL25 } & L245-DL25-95/05 & 95 & 05 & - & - \\
\cline { 2 - 6 } & L245-DL25-90/10 & 90 & 10 & - & - \\
\cline { 2 - 6 } & L245-DL25-85/15 & 85 & 15 & - & - \\
\cline { 2 - 6 } & L245-DL25-80/20 & 80 & 20 & - & - \\
\hline \multirow{2}{*}{ L245-DL70 } & L245-DL70-95/05 & 95 & - & 05 & - \\
\cline { 2 - 6 } & L245-DL70-90/10 & 90 & - & 10 & - \\
\hline
\end{tabular}




\begin{tabular}{|c|c|c|c|c|c|}
\hline & L245-DL70-85/15 & 85 & - & 15 & - \\
\cline { 2 - 6 } & L245-DL70-80/20 & 80 & - & 20 & - \\
\hline \multirow{2}{*}{ L245-SC } & L245-SC-99/01 & 99 & - & - & 01 \\
\cline { 2 - 6 } & L245-SC-95/05 & 95 & - & - & 05 \\
\hline \multirow{2}{*}{ L245-DL70-SC } & L245-DL70-SC-19/01 & 80 & - & 19 & 01 \\
\cline { 2 - 6 } & L245-DL70-SC-15/05 & 80 & - & 15 & 05 \\
\hline
\end{tabular}

\subsubsection{Thermal behaviour of blends}

To validate ideal mixing and its potential effect on L245 crystallization, the thermal behaviour of the prepared blends was studied by differential scanning calorimetry (DSC). Figure 3.4 shows the DSC curves obtained on cooling from the melt at $10^{\circ} \mathrm{C} / \mathrm{min}$. The $\mathrm{L} 245$ blends show typical semi-crystalline transitions for PLLA: melt crystallization $\left(T_{c}\right)$ and glass transition temperature $\left(\mathrm{T}_{\mathrm{g}}\right)$ that are given as a function of LMW PDLLA fraction in Figure 3.5(a). At a heating rate of $10^{\circ} \mathrm{C} / \mathrm{min}$, the glass transition of the LMW PDLLAs is $38^{\circ} \mathrm{C}$ and substantially lower than PLAs high in enantiomeric purity. Like most PLAs, L245 possesses a glass transition temperature of $64^{\circ} \mathrm{C}$. Albeit different compositions, all blends exhibit a single $T_{\mathrm{g}}$ that shifts to lower temperatures with increasing weight fraction $\mathrm{w}$ neatly following the Fox equation $1 / \mathrm{T}_{\mathrm{g}}=\left(\mathrm{w}_{1} / \mathrm{T}_{\mathrm{g} 1}\right)+\left(\mathrm{w}_{2} / \mathrm{T}_{\mathrm{g} 2}\right)$, indicating melt miscibility of PLLA and PDLLA. The exact glass transition and crystallization temperatures are given in Table B1 of Appendix B.

Like the glass transition temperature $\left(T_{g}\right)$, the crystallization temperature $\left(T_{c}\right)$ of both DL25 and DL70 L245 blends decreases gradually upon increasing PDLLA fraction. Up to 10 wt-\% of PDLLA additives, the normalized crystallization enthalpy upon cooling with $10^{\circ} \mathrm{C} / \mathrm{min}$ remains identical, namely $16-17 \mathrm{~J} / \mathrm{g}$ (Figure 3.5(b)). However, above a PDLLA weight percentage of $10 \mathrm{wt}$ \%, the normalized crystallization enthalpy, and thus L245 crystallinity, lowers significantly. As the exothermic peak broadens and extends towards the glass transition region, it is evident that the addition of LMW PDLLA chains retard crystallization. Here, it is important to specify the enantiomeric composition of the LMW poly(lactide) additives as enantiomerically pure LMW PLA additives that are identical to the matrix are known to promote crystallization ${ }^{34}$, but would be on the expense of molecular diffusion in weld formation. Even though blends with $20 \mathrm{wt}$ \% PDLLA (L245-DL70-80/20 and L245-DL25-80/20) barely crystallize at a cooling rate of $10^{\circ} \mathrm{C} / \mathrm{min}$, the addition of 1 wt-\% stereocomplex based LMW additives (L245-DL70-SC-19/01) leads to a distinct increase in both crystallization temperature and normalized crystallization enthalpy. Upon an increase in stereocomplex percentage to $5 \mathrm{wt}$ \% (L245-DL70-SC-15/05), an increase in both $\mathrm{T}_{c}$ and $\Delta \mathrm{H}_{\mathrm{mc}}$ is observed. Interestingly, on comparing blends containing SC crystals with and without the presence of DL70 (Figure 3.4(c) and (d)), it can be seen that the nucleation efficiency of SC crystals is higher in the presence of LMW PDLLA fraction (DL70). For instance, the same faction of SC crystal, PL245-SC-99/01 exhibits a lower $\mathrm{T}_{\mathrm{c}}$ and $\Delta \mathrm{H}_{\mathrm{mc}}$ than PL245-DL70- 
19/01. The high normalized enthalpy of the stereocrystal enriched samples also indicates that none of the L245 samples without stereocrystals crystallize to their maximum extent.

In the context of FDM, these results indicate that the retardation in crystallization, caused by the addition of LMW PDLLA fraction, promotes molecular diffusion across the weld interfaces but may induce geometrical instability. The addition of SC crystals accelerates the crystallization of the PLLA matrix, but whether actual improvement in weld stiffness is realized depends on (i) the viscosity of the matrix, (ii) process dependent heat management and (iii) crystallization rate - the topic of the subsequent sections.
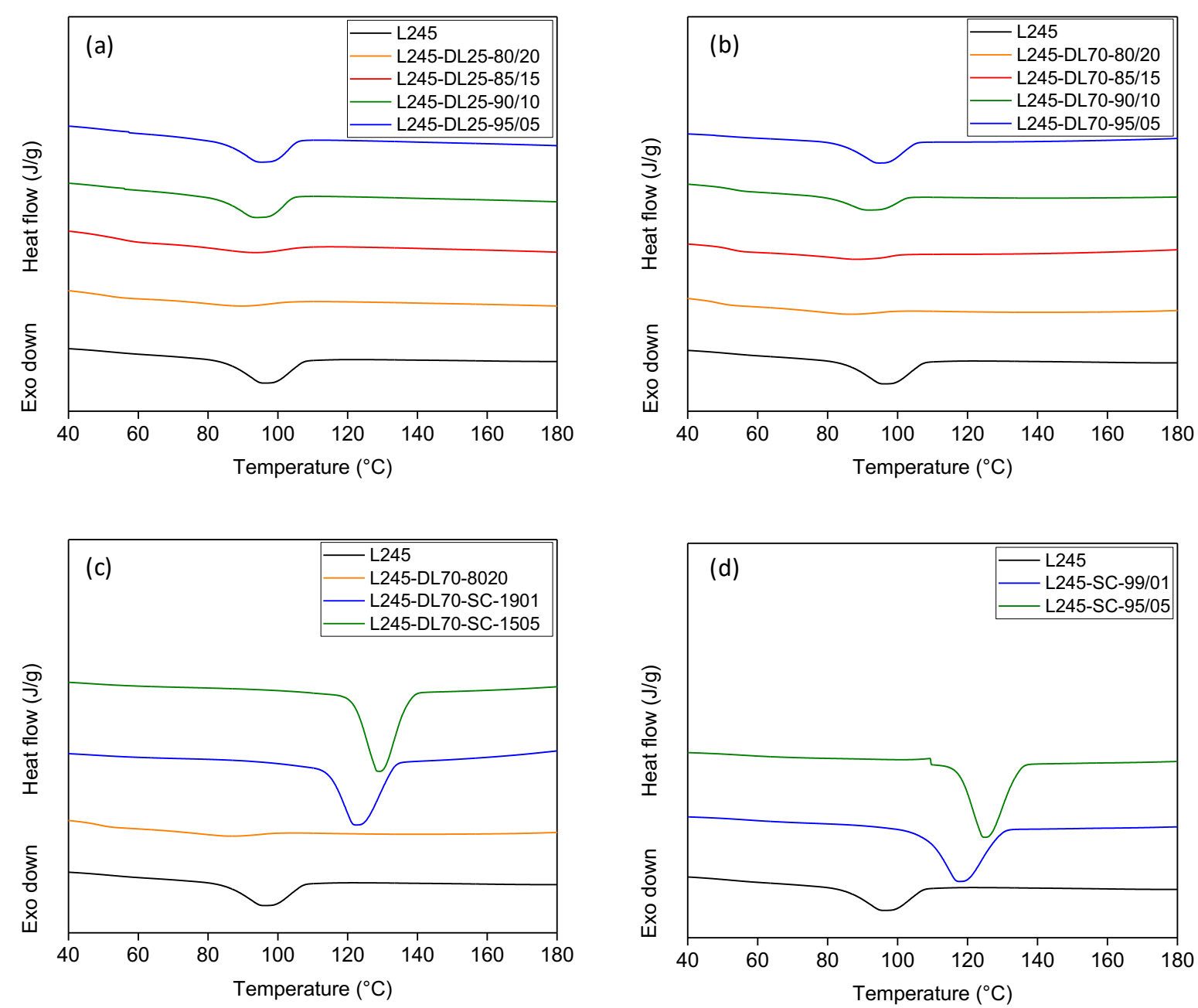

Figure 3.4: DSC thermograms based on cooling from $200^{\circ} \mathrm{C}$ at $10^{\circ} \mathrm{C} / \mathrm{min}$ for (a) L245-DL25, (b) L245-DL70 and (c) L245-DL7O-SC and (d) L245-SC bends. 

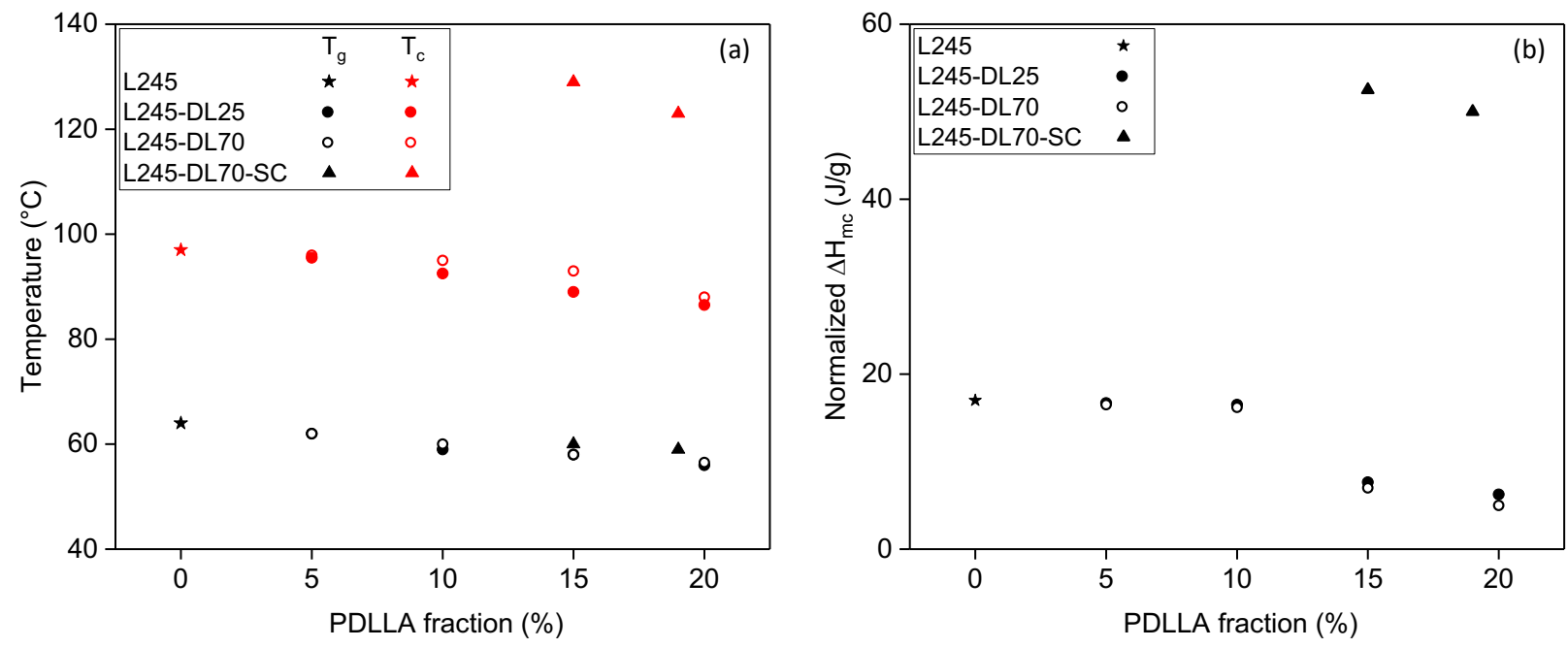

Figure 3.5: (a) Glass transition temperature $\left(T_{g}\right)$ and crystallization temperature $\left(T_{c}\right)$ plotted as a function of PDLLA fraction and (b) normalized crystallization enthalpy plotted as a function of PDLLA fraction for the studied blends. L245 represents the PLLA phase, L245-DL25 represents the blends containing DL25 as LMW fraction, L245-DL70 represents the blends containing DL70 as LMW fraction and L245-DL70-SC represents the blends containing DL70 and SC crystals.

\subsubsection{Melt rheology of blends}

To obtain mechanically robust interfaces, diffusion, mixing and entangling of chains across the weld interface is important. At the interface of layers, the weld is subjected to localized melting, where the interpenetration of chains occurs ${ }^{1,2}$. Melt rheology provides insights into the diffusivity of chains. Figure 3.6 depicts the complex viscosity of the blends as a function of angular frequency. Figure 3.6(a) and (b) show that an increase in LMW PDLLA fraction leads to a decrease in the complex viscosity that can be explained by the decrease in effective molar mass of the blend. Due to the macromolecular and chemically identical nature of the additives, the observed plasticization effect is predominantly effective in the melt state. Conventional plasticizers, on the contrary, are mostly low in molar mass and lower the glass transition substantially, often below or close to room temperature altering the mechanical properties like stiffness and yield stress in operation ${ }^{35-40}$. In this study, the selection of non-crystallizable LMW poly(lactides) lowers the complex viscosity to facilitate processing and welding while the glass transition temperature is reasonably retained.

In Figure 3.6(c) the effect of adding SC based LMW additives on the complex viscosity of L245 matrix can be seen. As SC crystals melt at a higher temperature than the PLLA homocrystals ${ }^{16,28,41}\left(\sim 170^{\circ} \mathrm{C}\right.$ for PLLA and $\sim 230^{\circ} \mathrm{C}$ for SC crystals), the SC based additive exists as semi-crystalline domains that are dispersed throughout the molten matrix at $180^{\circ} \mathrm{C}$ (Figure 3.3) and increase the complex viscosity. However, 1 wt-\% L245-SC-99/01 influences the complex viscosity and flow behaviour only slightly. On increasing the concentration to $5 \mathrm{wt}$-\% (L245-SC- 
95/05), the zero-shear complex viscosity increases by $28 \%$ with respect to L245 reaching the upper zero-shear viscosity limit. Although the intended nucleation may be beneficial in achieving spatial homogenization of crystallinity and thermodynamic/geometrical stability, flow behaviour and mechanically effective weld formation are likely hindered. To compensate, an additional class of blends was formulated combining the enhanced nucleation efficiency of SC based LMW additives with plasticization of the non-crystallizable LMW PDLLA additives, referred to as L245-DL70-SC. As in the non-plasticized case, the addition of $1 \mathrm{wt}-\%$ SC based LMW additive only slightly affects the complex viscosity and thus the flow behaviour. For $5 \mathrm{wt}-\%$ of SC based LMW additives, the complex viscosity increases by $200 \%$ with respect to the plasticized L245DL70-80/20 blend at $180^{\circ} \mathrm{C}$.
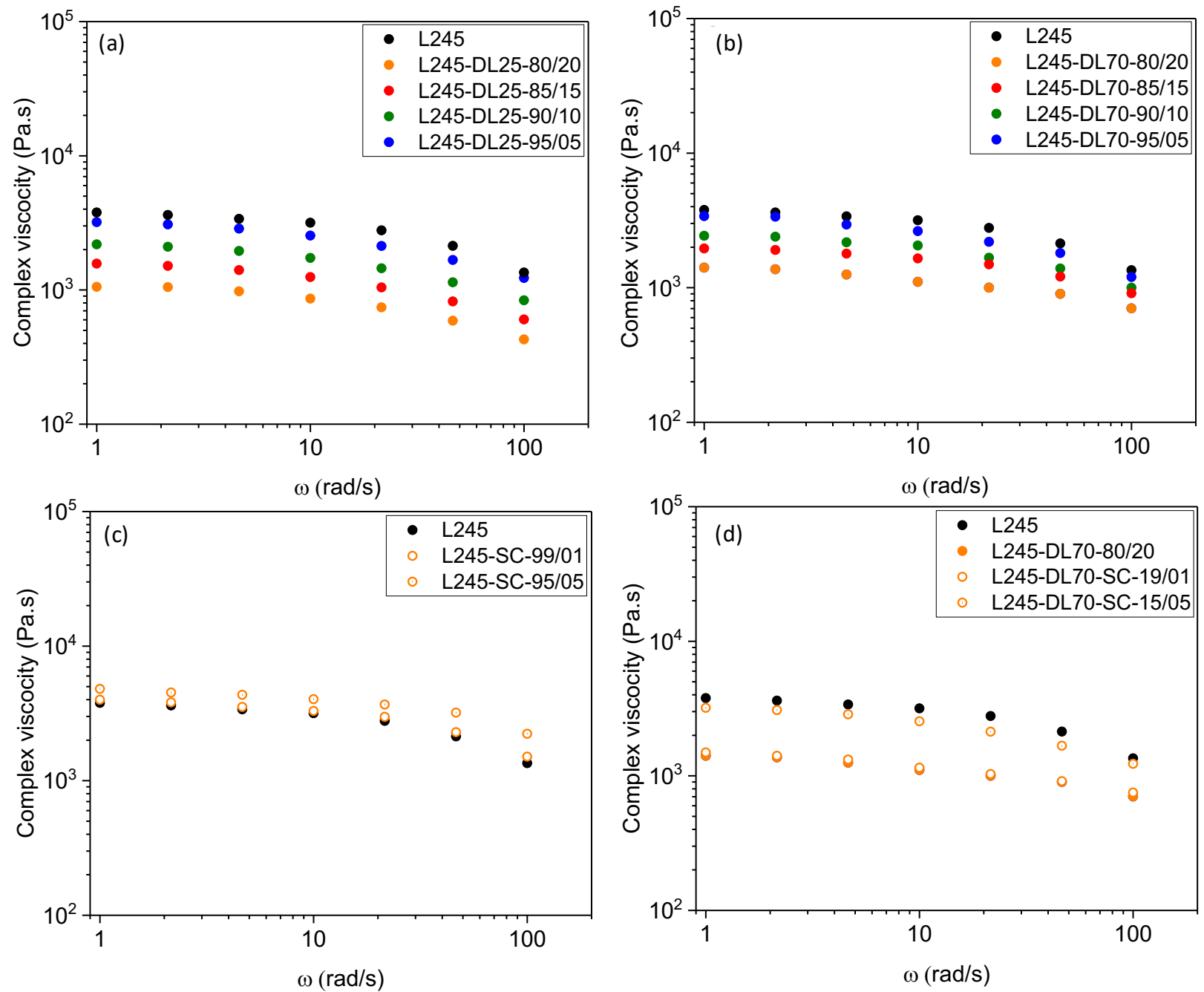

Figure 3.6: Complex viscosity plotted as a function of radial frequency for PDDLA (DL) plasticized (a) L245-DL25, (b) L245-DL70 PLA blends, and stereocrystal (SC) enriched (c) L245-SC and (d) L245-DL70-SC blends. 
How the induced changes in flow and crystallization behaviour affect chain diffusion and reentangling across weld interfaces depends on several factors. Firstly, as the entanglement molecular weight of PLLA is typically reported to be $4 \mathrm{~kg} / \mathrm{mol}{ }^{44}$ - ideally - single chains of DL 25 and DL70 can only engage in form 5 and 16 entanglements respectively. Secondly, as seen in Figure 3.6(a) and (b) the viscosity scales inversely with the fraction of DL25 or DL70. Thus, under the same processing history, higher low molecular weight fractions should promote more interpenetration of chains at the interface. Thirdly, the effect of the additives on the onset of PLA L245 matrix crystallization and chain diffusion needs to be considered in the context of the complex heat management in FDM printing that is discussed in the next section.

\subsubsection{Heat management and crystallization in FDM}

An ultrathin thermocouple was embedded in the build plate to record the temperature of the first layer during the entire printing process. The temperature profile imposed on the first layer for a print speed of $20 \mathrm{~mm} / \mathrm{s}$ is depicted in Figure 3.7(a) and (b). Each time the nozzle passes over the thermocouple, a sharp increase in temperature is seen. Moreover, interesting trends are observed based on the peak maxima and minima as a function of time. Firstly, the highest increase in temperature (first peak maximum) is seen for the first layer and decreases on the deposition of subsequent layers. After approximately 75 seconds, the peaks are no longer observable and the temperature gradually reduces to the set ambient temperature of $70^{\circ} \mathrm{C}$. Secondly, under these print conditions, the peak minima increase initially to temperatures well above $70^{\circ} \mathrm{C}$, meaning that not all administered heat is dissipated to the surroundings. The generated temperature profiles are overlaid by the crystallization onset times $\left(t_{0}\right)$ for isothermal melt crystallization (MC) and cold crystallization (CC) at different temperatures. The onset times have been derived from isothermal measurements at different crystallization temperatures and is embedded in Appendix B, Figure B4. To understand how molecular diffusion and crystallization proceed towards part completion in FDM, the distinction between melt and cold crystallization is pivotal. This methodological alignment of FDM specific heat management and crystallization rates have proven to be effective in understanding the accessible time-scales for molecular diffusion and completion of crystallization ${ }^{3}$. If due to printing speed and geometrical design (which are collectively represented by the heating profiles), crystallization in melt deposition is (partly) suppressed by approaching the glass transition temperature, the poly(lactides) will "cold crystallize" upon reheating by an additively deposited melt. ${ }^{3}$

In line with the results of section 3.1 , both the addition and increasing molar mass of the noncrystallizable LMW PDLLA the onset time of melt and cold crystallization and thus the time for molecular diffusion in weld formation increase, Figure 3.5(a). Furthermore, the intersection of the temperature profile with the crystallization curves suggests that melt crystallization during initial deposition (the first sharp temperature decline starting at $140^{\circ} \mathrm{C}$ ) does not occur for any 
of the blends studied. As the rate of cold crystallization is faster, crystallization is expected to proceed primarily via cold crystallization. Nevertheless, the addition of the PDLLA of $70 \mathrm{~kg} / \mathrm{mol}$ reduces the crystallization rate to such an extent that crystallization during printing may not occur at all.
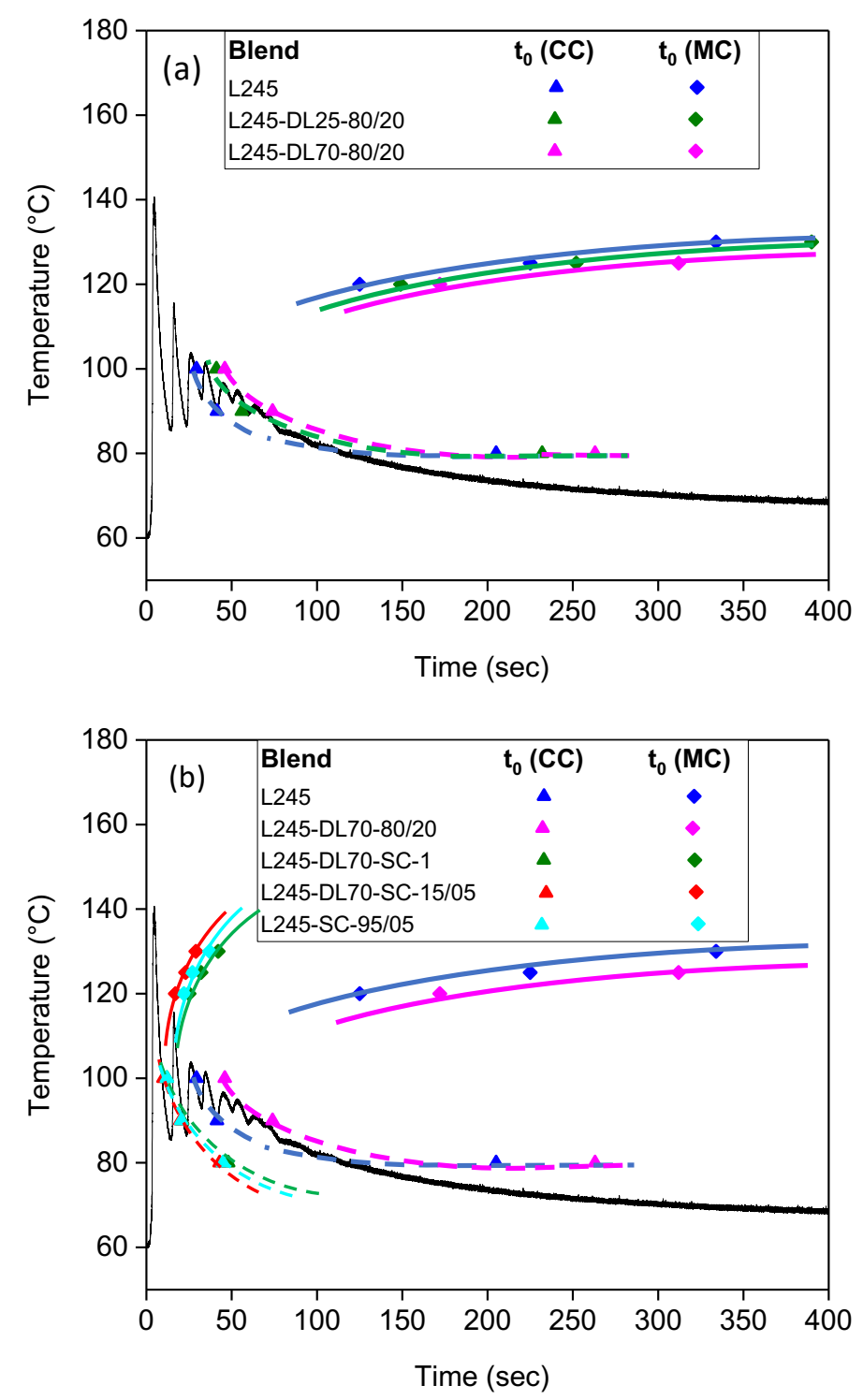

Figure 3.7: Temperature profile measured at the center of the bottom layer at a print speed of $20 \mathrm{~mm} / \mathrm{s}$ overlaid by the isothermal crystallization onset times for melt crystallization (solid) and cold crystallization (dotted) for (a) PLLA/PDLLA (DL) blends with 20 wt-\% PDLLA of either 25 or $70 \mathrm{~kg} / \mathrm{mol}$, and (b) stereocomplex enriched PLLA-PDLLA-SC blends (DL-SC and SC) against pure and a PDLLA plasticized PLLA 245 (L245 and L245-DL70 respectively).

In Figure 3.7(b) the crystallization onset times $\left(t_{0}\right)$ for isothermal melt crystallization $(M C)$ and cold crystallization (CC) have also been overlaid with the specific temperature profile for blends containing the stereocomplex based additives crystals with and without LMW DL70. As expected, a drastic reduction in the onset time of melt and cold crystallization is observed. Intersection -50 - 
of the temperature profile with the melt and cold crystallization curves (solid/dashed red and green lines) suggests that crystallization during initial deposition occurs. Based on the findings of the previous chapter ${ }^{3}$, upon crystallization, molecular diffusion across layer interfaces arrests for PLA. However, the presence of (i) a mobile non-crystallizable phase witnessed by the reduced complex viscosity and (ii) sufficient reheating and melting of a previously deposited layer in the L245-DL70-SC samples, may improve interfacial diffusion despite the enhanced crystallization rate. To recall, in the previous section, we found that even though SC crystals are present up to $5 \mathrm{wt}$-\%, the zero-shear viscosity at $180^{\circ} \mathrm{C}$ is lower than that of $\mathrm{L} 245$ at the same temperature. Additionally, the presence of SC crystals will also promote the crystallization of the PLLA. How potential diffusion, entangling and crystallization of the LMW modified PLA L245 blends influences interfacial bonding is elucidated into the forthcoming section, where the interfacial stiffness of printed parts is evaluated.

\subsubsection{Interfacial Mechanical properties of 3D printed parts}

In the absence of crystals, the mechanical integrity of FDM weld interfaces is determined by the number of entanglements per chain formed upon interfacial diffusion ${ }^{1,23,43}$. Additionally, it has been shown that the presence of crystals specifically directed at the interface by alternating deposition of enantiomerically opposite poly(lactides), significantly enhances the interfacial stiffness and thermo-mechanical performance ${ }^{9}$. Torsional oscillatory DMA in a temperature range from $40^{\circ} \mathrm{C}$ to $120^{\circ} \mathrm{C}$, specifically transferring the stress to the interface, was conducted to study the effect of LMW PDLLA induced melt plasticization and nucleation of stereocomplex based additives on weld mechanics, Figure 3.8. For the plasticized PLA245-DL25 and PLA245DL70 series, only the samples containing $20 \mathrm{wt}$ \% LMW fraction are depicted as they exhibit the lowest crystallization rate. Above the glass transition, the reference material L245 shows a considerable drop and successive increase in storage modulus around $90^{\circ} \mathrm{C}$ due to cold crystallization, indicating low crystallinity after printing. For blends containing PDLLA LMW fractions (L245-DL25-80/20 and L245-DL70-80/20), the drop in storage modulus is even larger, meaning that the crystallinity attained after printing is lower than the reference L245. In fact, upon increasing molar mass of the non-crystallizable PDLLA fraction, the initial crystallinity progressively lowers as predicted by the intersections of the temperature profile and isothermal crystallization curves, Figure 3.7. Also, the plasticization effect of the LMW PDLLAs is evident and follows the Fox equation as observed by DSC. On the contrary, in blends containing SC crystals (L245-SC-99/01 and L245-DL70-SC-19/01) no reorganization is seen above the glass transition temperature proving that the addition of $1 \mathrm{wt}-\% \mathrm{SC}$ based LMW additive is sufficient to achieve maximum crystallinity and thus a thermodynamically and thus geometrically stable state. The above results are corroborated by polarized optical micrographs that are embedded in Figure B5 of Appendix B. 
In Figure 3.9(a), the storage modulus $\left(G^{\prime}\right)$ at $40^{\circ} \mathrm{C}$ is plotted as a function of PDLLA fraction (DL25 or DL70) for compression molded and fused deposition modeled samples. The storage modulus of the compression molded samples represents the intrinsic bulk stiffness of the various blends. The samples were prepared in a mold of $20 \times 5 \times 1 \mathrm{~mm}^{3}$ in dimension and operating at $180^{\circ} \mathrm{C}$, from where the samples were quenched in acetone of $-80^{\circ} \mathrm{C}$ to ensure a fully amorphous state. For all compression molded samples containing LMW PDLLA additives (L245-DL70 and L245-DL25 blends), the storage modulus is only slightly negatively affected by the addition of LMW species. This is expected as the effective molecular weight of these blends is well above the entanglement molecular weight of PLA. It has been observed that once a critical number of entanglements have been achieved, an increase in stiffness with molecular weight is limited and a plateau is reached ${ }^{15,44}$.

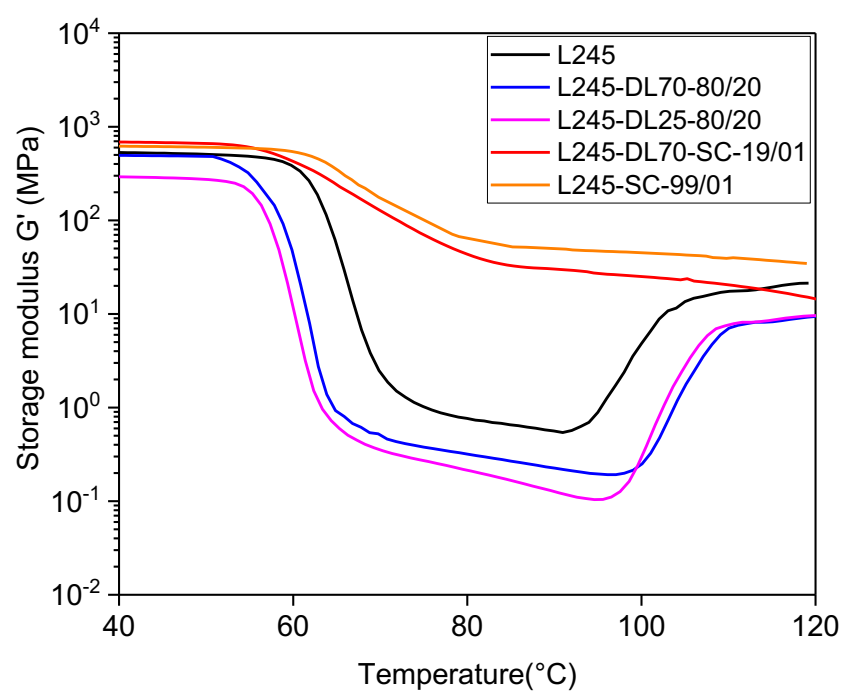

Figure 3.8: Torsional storage modulus $G$ ' plotted as a function of temperature for L245, plasticized L245-DL70-80/20 and L245-DL25-80/20 possessing both $20 \mathrm{wt}-\%$ of LMW PDLLA (DL), and stereocomplex (SC) enriched L245-DL70-SC-19/01 and L245-SC-99/01 blends.

However, under FDM conditions, trends are observed on varying the PDLLA concentration and molecular weight (Figure 3.9(a)). For the L245-DL25 blends, the modulus remains constant for concentrations up to $15 \mathrm{wt}$ \% and is slightly lower than the modulus of the reference material (L245). Above $15 \mathrm{wt}$ \% of DL70 a sharp decrease in modulus is observed. The blend containing 20 wt-\% of DL25 has a modulus of almost $40 \%$ lower than the printed reference sample. Even though DL25 provides the highest diffusivity, it has no positive impact on the interfacial stiffness, which is likely due to the lack of a new mechanically functional entanglement network across the interface. Note that the molecular weight of DL25 is only about 6 times the molecular weight between entanglements for PLLA. At a very high concentration of DL25 the sharp 
decrease in modulus, which is disproportionally larger than in the case of the molded samples, furthermore, suggests a high fraction of low molecular weight chains at the interface due to flow-induced fractionation.

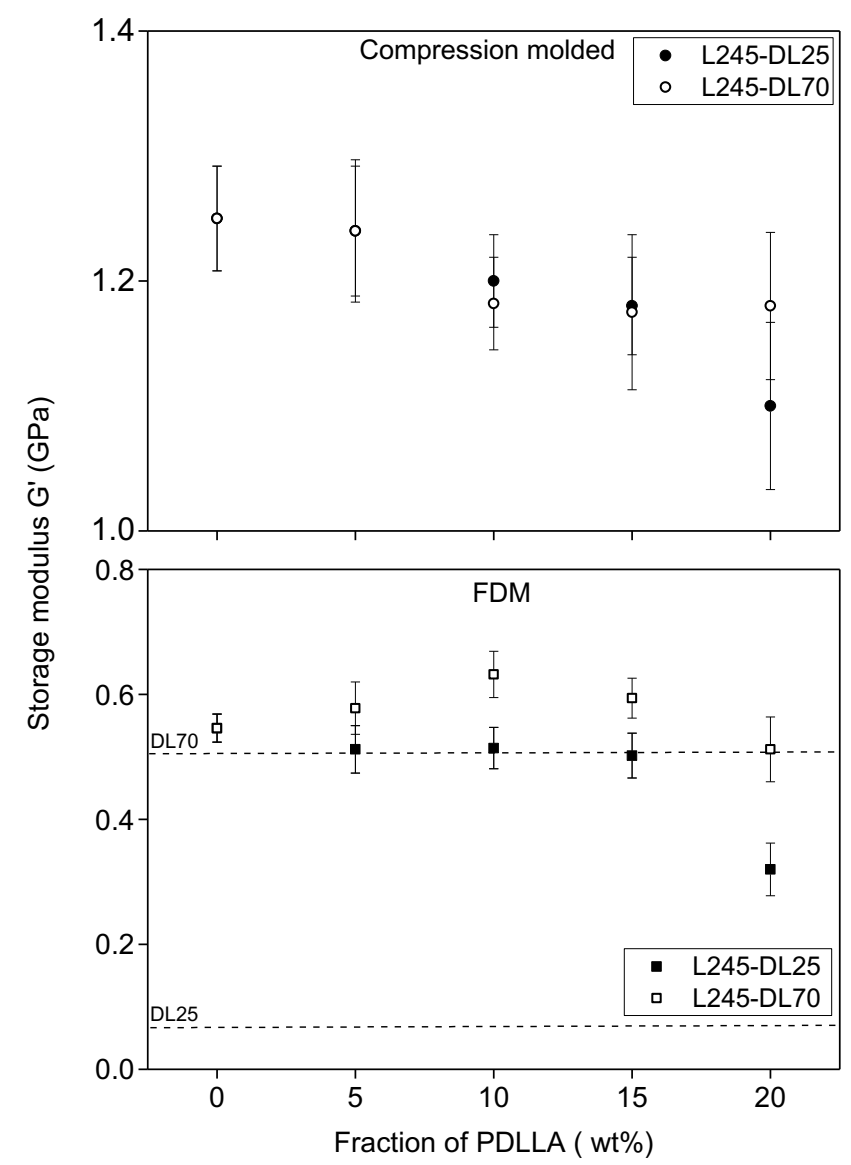

(a)

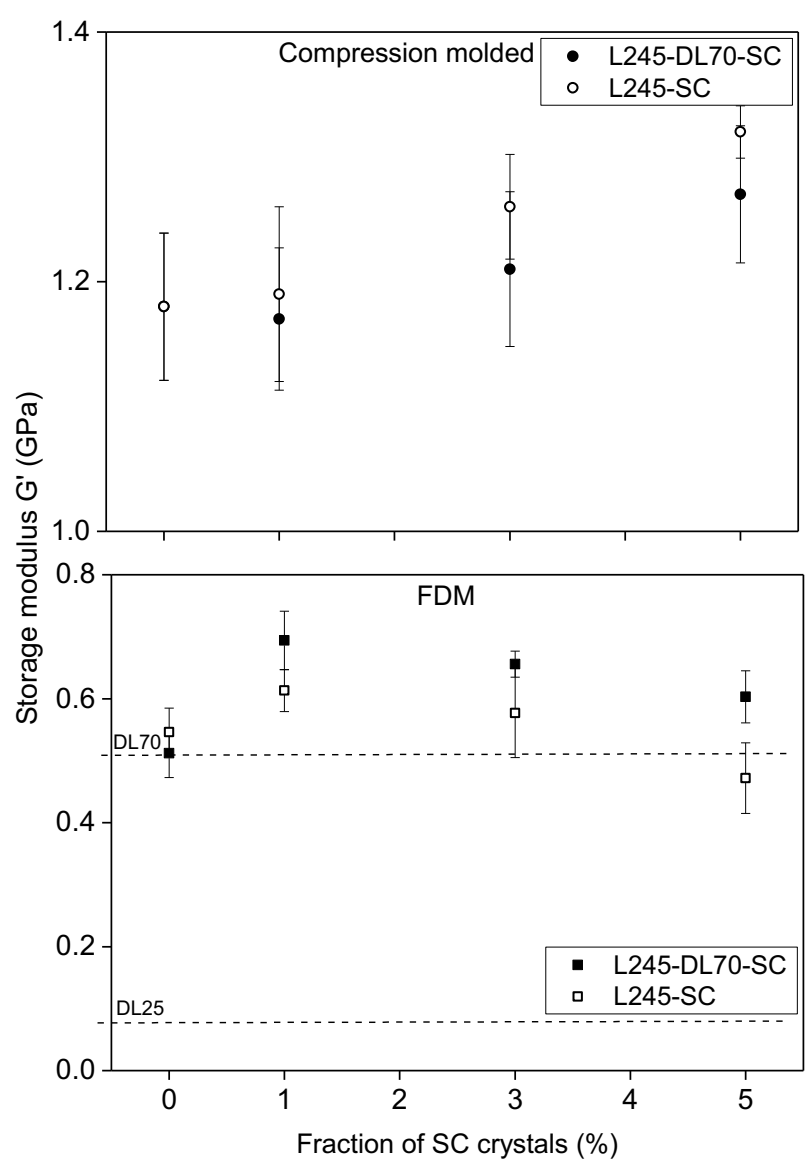

(b)

Figure 3.9: Torsional storage modulus at $40^{\circ} \mathrm{C}$ for (a) plasticized blends containing low MW PDLLA (DL25 or DL70) and (b) blends containing stereocomplex (SC) with (L245-DL-SC) or without plasticizing DL70 (L245- SC blends). In (a) and (b) the dotted lines represent the bulk stiffness measured on compression molded DL25 and DL70 respectively.

Interestingly, a different trend is observed for the increasing weight fraction of L245-DL70 in the blends. Within the studied range of LMW additives, the modulus of the compression molded samples is not affected. For the FDM samples, concentrations up to $10 \mathrm{wt}$ \% enhance the storage modulus from $0.546 \mathrm{GPa}$ to $0.632 \mathrm{GPa}$, corresponding to a roughly $15 \%$ increase in stiffness. At concentrations above $10 \mathrm{wt} \%$, the gain in properties due to the presence of DL70 chains diminishes and is likely caused by the excess of DL70 chains at the interface. It appears that it is vitally important that molecular weight and concentration are balanced to achieve any mechanical stiffening. It should also be noted that under these printing conditions, the 
influence of PLLA crystallization on the interface stiffness can be neglected. All FDM samples based on L245-DL25 and L245-DL70 blends are highly amorphous, as proven by Figure 3.8.

In Figure 3.9(b) the storage modulus of compression molded and FDM printed samples are plotted upon increasing weight fraction of SC based LMW additives for L245-SC and L245-DL70SC blends. To recall, the presence of stereocrystals entails molded and FDM samples that are fully crystalline in nature. On the one hand, crystallization can be beneficial to increase the stiffness, but as the crystallization already begins before the deposition of a new additive layer, Figure 3.7(b), the crystalline phase of the previous layer at the interface must melt to facilitate molecular diffusion.

For L245-SC blends prepared via compression molding, the stiffness slightly increases on increasing the fraction of SC based LMW additives in the blend. The effect can be attributed to the presence of solid SC crystals ${ }^{27,45}$. For samples prepared via FDM, it is observed that for 1 wt-\% SC based LMW additives, the stiffness is higher than the reference material by almost $15 \%$ clearly indicating that crystals contribute to the realized stiffness. Here, crystals provide reinforcement, even though diffusion would be hindered. For blends with higher concentrations of SC based LMW additives, the hindrance to diffusion becomes more prominent, likely explaining the decrease in stiffness while the crystallinity for all samples is equally high. In fact, for the blend with $5 \mathrm{wt}$-\% SC based LMW additive, the storage modulus is lower than that of the reference material L245. Another blend with $3 \mathrm{wt}$ \% SC based LMW additives was prepared, but already at this concentration a decline in stiffness is observed. The progressive increase in crystallization rate upon increasing the fraction of SC based LMW additives, Figure 3.5(b), clearly hinders interlayer diffusion and mechanical reinforcement of the weld interface but secures thermodynamic and geometrical stability.

Since the LMW PDLLA additives cannot crystallize, they may provide sufficient diffusion to the SC nucleated bends. In Figure 3.9(b) the interfacial stiffness of L245-DL70-SC blends is given with increasing fraction of SC based LMW additives. Like observed before, the presence of stereocrystals causes an increase in the stiffness of the compression molded samples. Under FDM conditions, the blend containing $1 \mathrm{wt}-\%$ SC based LMW additive (L245-DL70-SC-19/01) shows a substantial $28 \%$ increase in stiffness as compared to the reference material. In comparison, the corresponding blend without the non-crystallizable DL70 (L245-SC-99/01) shows only an improvement of $15 \%$, as discussed earlier. Upon increasing the fraction of SC crystals the stiffness decreases, which can be attributed to the SC induced (i) increased overall crystallization rate (Figure 3.5(b)) and/or (ii) increased melt viscosity (Figure 3.6(c)) that both restrict diffusion of chains across the weld interfaces. 


\subsection{CONCLUSIONS}

To mechanically improve fused deposition modeled weld interfaces, the influence of enantiomeric composition and relative weight fractions of multimodal poly(lactide) blends on molecular diffusion and crystallization in weld formation has been systematically and selectively studied independently from shape. Based on the judicious selection of the enantiomeric composition of particularly the low molar mass fraction(s), interfacial molecular diffusion and/or co-crystallization of polylactide chains physically anchoring chains across the interface are promoted.

Albeit possessing the identical chemistry, the random enantiomeric composition of low molar mass PDLLA additives slightly lowers the glass transition temperature of the resulting blend. The trend in the glass transition temperature follows the Fox equation, implying full miscibility. It is particularly the complex viscosity of the melt that decreases substantially and, that in combination with reduced crystallization rate, enables molecular diffusion at weld interfaces. The low amount of entanglements per low molar mass chains leads to poor interfacial stiffening. However, if the molar mass of the non-crystallizable low molar mass PDLLA additive increases, the crystallization rate is reduced to such an extent that ultimate completion of crystallization and thus geometrical stability will not be realized. While the intrinsic torsional stiffness of the blends barely changes with the addition of non-crystallizable low molar mass fractions, the weld stiffness of fused deposition modeled parts lowers with too high concentrations of noncrystallizable PDLLA. Only for a reasonably high molar mass and low weight fractions of the noncrystallizable molar mass, a $15 \%$ stiffening of the weld interface is observed.

The need for interfacial co-crystallization where chains of successively deposited strands are physically anchored by individual crystals is evident from a mechanical perspective. However, the addition of solely stereocomplex based low molar mass additives results in well-dispersed semi-crystalline domains that increase the crystallization rate to the moment of melt deposition. Thereby, challenging printability, interfacial molecular diffusion and mechanical reinforcement of the weld interface but securing complete crystallization and thermodynamic stability.

On combining both additives by selecting the higher molar mass non-crystallizable low molecular weight PDLLA, the zero-shear viscosity of the melt reduces considerably although the crystallization rate of the blends remains high due to the stereocomplex nucleation and complete crystallization throughout the entire fused deposition modeled object is observed. The plasticization effect of the non-crystallizable low molar mass PDLLA promotes molecular diffusion and mixing in weld formation, albeit the presence of nucleating stereocrystals. The engagement of polymer chains originating from both sides of the interface in a semi-crystalline 
network upon weld formation distinctly increases ultimate weld stiffness and thermodynamic/geometrical stability of fused deposition modeled poly(lactide) blends. 


\section{REFERENCES}

(1) Mcllroy, C.; Olmsted, P. D., Disentanglement Effects on Welding Behaviour of Polymer Melts during the Fused-Filament-Fabrication Method for Additive Manufacturing. Polymer. 2017, 123, 376-391.

(2) Mcllroy, C.; Olmsted, P. D., Deformation of an Amorphous Polymer during the Fused-FilamentFabrication Method for Additive Manufacturing. Journal of Rheology. 2017, 61 (2), 379-397.

(3) Seppala, J. E.; Hoon Han, S.; Hillgartner, K. E.; Davis, C. S.; Migler, K. B., Weld Formation during Material Extrusion Additive Manufacturing. Soft Matter. 2017, 13 (38).

(4) Sweeney, C. B.; Lackey, B. A.; Pospisil, M. J.; Achee, T. C.; Hicks, V. K.; Moran, A. G.; Teipel, B. R.; Saed, M. A.; Green, M. J., Welding of 3D-Printed Carbon Nanotube-polymer Composites by Locally Induced Microwave Heating. Science Advances. 2017, 3 (6), e1700262.

(5) Meijer, H. E. H.; Govaert, L. E., Mechanical Performance of Polymer Systems: The Relation between Structure and Properties. In Progress in Polymer Science; 2005, 30, 915-938.

(6) Srinivas, V.; van Hooy-Corstjens, C. S. J.; Harings, J. A. W., Correlating Molecular and Crystallization Dynamics to Macroscopic Fusion and Thermodynamic Stability in Fused Deposition Modeling; a Model Study on Polylactides. Polymer. 2018, 142, 348-355.

(7) Mcllroy, C.; Graham, R. S., Modelling Flow-Enhanced Crystallisation during Fused Filament Fabrication of Semi-Crystalline Polymer Melts. Additive Manufacturing. 2018, 24, 323-340.

(8) Panda, B. N.; Shankhwar, K.; Garg, A.; Jian, Z., Performance Evaluation of Warping Characteristic of Fused Deposition Modelling Process. Internation Journal of Advanced Manufacturing Technology. 2017, 88 (5), 1799-1811.

(9) Srinivas, V.; van Hooy-Corstjens, C. S. J.; Vaughan, G. B. M.; van Leeuwen, B.; Rastogi, S.; Harings, J. A. W., Interfacial Stereocomplexation To Strengthen Fused Deposition Modeled Poly(Lactide) Welds. ACS Applied Polymer Materials. 2019, 1 (8), 2131-2139.

(10) Di Lorenzo, M. L., Determination of Spherulite Growth Rates of Poly(l-Lactic Acid) Using Combined Isothermal and Non-Isothermal Procedures. Polymer. 2001, 42 (23), 9441-9446.

(11) Li, X.-J.; Li, Z.-M.; Zhong, Gan.-J.; Li L.-B., Steady-Shear-Induced Isothermal Crystallization of Poly(L-Lactide) (PLLA). Journal of Macromolecular Science, Part B. 2008, 47 (3), 511-522.

(12) Harris, A. M.; Lee, E. C., Improving Mechanical Performance of Injection Molded PLA by Controlling Crystallinity. Journal of Applied Polymer Science. 2008, 107 (4), 2246-2255.

(13) Seo, Y.; Kim, J.; Kim, K. U.; Kim, Y. C., Study of the Crystallization Behaviors of Polypropylene and Maleic Anhydride Grafted Polypropylene. Polymer. 2000, 41 (7), 2639-2646.

(14) Pei, A.; Zhou, Q.; Berglund, L. A., Functionalized Cellulose Nanocrystals as Biobased Nucleation Agents in Poly(l-Lactide) (PLLA) - Crystallization and Mechanical Property Effects. Composite Science and Technology. 2010, 70 (5), 815-821.

(15) Perego, G.; Cella, G. D.; Bastioli, C., Effect of Molecular Weight and Crystallinity on Poly(Lactic Acid) Mechanical Properties. Journal of Applied Polymer Science. 1996, 59 (1), 37-43.

(16) Saeidlou, S.; Huneault, M. A.; Li, H.; Park, C. B., Poly(Lactic Acid) Crystallization. Progress in Polymer Science. 2012, 37 (12), 1657-1677.

(17) Bustos, F.; Cassagnau, P.; Fulchiron, R., Effect of Molecular Architecture on Quiescent and ShearInduced Crystallization of Polyethylene. Journal of Polymer Science Part B: Polymer Physics. 2006, 44 (11), 1597-1607.

(18) Lanzotti, A.; Grasso, M.; Staiano, G.; Martorelli, M., The Impact of Process Parameters on Mechanical Properties of Parts Fabricated in PLA with an Open-Source 3-D Printer. Rapid Prototyping Journal. 2015, 21 (5), 604-617.

(19) Agarwala, M. K.; Jamalabad, V. R.; Langrana, N. A.; Safari, A.; Whalen, P. J.; Danforth, S. C., Structural Quality of Parts Processed by Fused Deposition. Rapid Prototyping Journal. 1996, 2 (4), 4-19.

(20) Sood, A. K.; Ohdar, R. K.; Mahapatra, S. S., Parametric Appraisal of Mechanical Property of Fused Deposition Modelling Processed Parts. Materials and Design. 2010, 31 (1), 287-295.

(21) Wang, L.; Gardner, D. J., Effect of Fused Layer Modeling (FLM) Processing Parameters on Impact Strength of Cellular Polypropylene. Polymer. 2017, 113, 74-80.

(22) Meng, S.; He, H.; Jia, Y.; Yu, P.; Huang, B.; Chen, J., Effect of Nanoparticles on the Mechanical Properties of Acrylonitrile-Butadiene-Styrene Specimens Fabricated by Fused Deposition Modeling. Journal of Applied Polymer Science. 2017, 134 (7).

(23) Levenhagen, N. P.; Dadmun, M. D., Bimodal Molecular Weight Samples Improve the Isotropy of 3D Printed Polymeric Samples. Polymer. 2017, 122, 232-241.

(24) Musil, J.; Zatloukal, M., Experimental Investigation of Flow Induced Molecular Weight Fractionation during Extrusion of HDPE Polymer Melts. Chemical Engineering and Science. 2011, 66 (20), 4814-4823.

(25) Musil, J.; Zatloukal, M., Flow Induced Molecular Weight Fractionation during Capillary Flow of 
Linear Polymer Melt, Recent Advances in Fluid Mechanics, Heat \& Mass Transfer and BiologyProceedings of the 9th WSEAS International Conference on Fluids. 2012, 162-167.

(26) Levenhagen, N. P.; Dadmun, M. D., Interlayer Diffusion of Surface Segregating Additives to Improve the Isotropy of Fused Deposition Modeling Products. Polymer. 2018, 152, 35-41.

(27) Tsuji, H.; Bouapao, L. Stereocomplex Formation between Poly(L-Lactic Acid) and Poly(D-Lactic Acid) with Disproportionately Low and High Molecular Weights from the Melt. Polymer International. 2012, 61 (3), 442-450.

(28) Ikada, Y.; Jamshidi, K.; Tsuji, H.; Hyon, S. H., Stereocomplex Formation between Enantiomeric Poly(Lactides). Macromolecules. 1987, 20 (4), 904-906.

(29) Tsuji, H.; Ikada, Y., Stereocomplex Formation between Enantiomeric Poly(Lactic Acid)s. XI. Mechanical Properties and Morphology of Solution-Cast Films. Polymer. 1999, 40 (24), 6699-6708.

(30) Saeidlou, S.; Huneault, M. A.; Li, H.; Park, C. B., Effect of Nucleation and Plasticization on the Stereocomplex Formation between Enantiomeric Poly(Lactic Acid)S. Polymer. 2013, 54 (21), 57625770.

(31) Tsuji, H., Poly(Lactic Acid) Stereocomplexes: A Decade of Progress. Advanced Drug Delivery Reviews. 2016, 107, 97-135.

(32) Tsuji, H.; Takai, H.; Saha, S. K., Isothermal and Non-Isothermal Crystallization Behavior of Poly(lLactic Acid): Effects of Stereocomplex as Nucleating Agent. Polymer. 2006, 47 (11), 3826-3837.

(33) Schmidt, S. C.; Hillmyer, M. A., Polylactide Stereocomplex Crystallites as Nucleating Agents for Isotactic Polylactide. Journal of Polymer Science Part B: Polymer Physics. 2001, 39 (3), 300-313.

(34) Di Lorenzo, M. L.; Androsch, R., Crystallization Kinetics of Blends of Two Poly(Lactic Acid) Grades with Diverse Stereoregularity and Molar Mass. AIP Conference Proceedings;, 2018 ; 981.

(35) Kulinski, Z.; Piorkowska, E., Crystallization, Structure and Properties of Plasticized Poly(lLactide). Polymer. 2005, 46 (23), 10290-10300.

(36) Ljungberg, N.; Colombini, D.; Wesslen, B., Plasticization of Poly(Lactic Acid) with Oligomeric Malonate Esteramides: Dynamic Mechanical and Thermal Film Properties. Journal of Applied Polymer Science, 2005, 96 (4), 992-1002.

(37) Ren, Z.; Dong, L.; Yang, Y., Dynamic Mechanical and Thermal Properties of Plasticized Poly(Lactic Acid). Journal of Applied Polymer Science, 2006, 101 (3), 1583-1590.

(38) Ljungberg, N.; Wesslén, B. Preparation and Properties of Plasticized Poly(Lactic Acid) Films. Biomacromolecules. 2005, 6 (3), 1789-1796.

(39) Jacobsen, S.; Fritz, H. G., Plasticizing Polylactide: The Effect of Different Plasticizers on the Mechanical Properties. Polymer Enggineering and Science. 1999, 39 (7), 1303-1310.

(40) Auras, R. A.; Lim, L. T.; Selke, S. E. M.; Tsuji, H., Poly(Lactic Acid): Synthesis, Structures, Properties, Processing, and Applications; Wiley, 2011.

(41) Tsuji, H.; Ikada, Y.; Hyon, S.-H.; Kimura, Y.; Kitao, T., Stereocomplex Formation between Enantiomeric Poly(Lactic Acid). VIII. Complex Fibers Spun from Mixed Solution of Poly(D-Lactic Acid) and Poly(L-Lactic Acid). Journal of Appled Polymer Science. 1994, 51 (2), 337-344.

(42) Dorgan, J. R.; Williams, J. S.; Lewis, D. N., Melt Rheology of Poly(Lactic Acid): Entanglement and Chain Architecture Effects. Jorunal of Rheology. 1999, 43 (5), 1141-1155.

(43) Levenhagen, N. P.; Dadmun, M. D. Improving Interlayer Adhesion in 3D Printing with Surface Segregating Additives: Improving the Isotropy of Acrylonitrile-Butadiene-Styrene Parts. ACS Applied Polymer Materials. 2019, 1 (4), 876-884.

(44) Nunes, R. W.; Martin, J. R.; Johnson, J. F., Influence of Molecular Weight and Molecular Weight Distribution on Mechanical Properties of Polymers. Polymer Engineering and Science. 1982, 22 (4), 205-228.

(45) Tan, B. H.; Muiruri, J. K.; Li, Z.; He, C., Recent Progress in Using Stereocomplexation for Enhancement of Thermal and Mechanical Property of Polylactide. ACS Sustainable Chemistry and Engineering. 2016, 4 (10), 5370-5391. 


\section{Chapter 4}

\section{Interfacial stereocomplexation to strengthen fused deposition modeled poly(lactide) welds}

This chapter is based on the following publication:

Srinivas, V.; van Hooy-Corstjens, C. S. J.; Vaughan, G. B. M.; van Leeuwen, B.; Rastogi, S.; Harings, J. A. W. Interfacial Stereocomplexation To Strengthen Fused Deposition Modeled Poly(Lactide) Welds. ACS Applied Polymer Materials. 2019, 1 (8), 2131-2139. 


\begin{abstract}
The inter-layer stiffness of fused deposition modeled (FDM) parts is significantly impaired by the slow diffusion and re-entangling of polymer chains across filament interfacial welds in the build direction. To mechanically reinforce FDM interfaces, a novel approach based on timing and spatial direction of stereocomplexation across weld interfaces is introduced via alternating deposition of enantiomerically opposite poly(lactides). Fundamental insight in the kinetics and spatial distribution of homo- and stereocrystallization at FDM interfaces was successfully reconstructed in 2 and 3 dimensions by micrometer resolved Fourier transform infrared microscopy and synchrotron wide angle X-ray diffraction tomography. The rate of isothermal stereocomplexation and consequential interfacial stiffening increases with decreased absolute and relative molar masses. The spatial distribution of stereocrystals is governed by the relative molar masses and the extent of inter-diffusion, not only under isothermal but also nonisothermal FDM conditions. The net local heat dosage, which depends on print speed, governs the length-scales of stereocomplexation and thus mechanical reinforcement. Interfacial stereocomplexation of poly(lactides) in FDM leads to a distinct $40 \%$ increase in stiffness and nucleation of bulk filaments, aiding in thermodynamic and geometrical stability.
\end{abstract}




\subsection{INTRODUCTION}

In recent years, in an effort to create mechanically more robust printed objects, extensive research has been carried out on the influence of FDM process conditions on the mechanical properties, particularly focusing at layer interfaces. Some of these conditions/parameters include deposition strategy ${ }^{11,12}$, filament to filament air gap ${ }^{13}$, layer height, and bed temperature ${ }^{11}$. Yet, these strategies improve the mechanical properties only to a certain extent and the necessity for more improvement prevails. Meanwhile, other researchers studied the problem from the perspective of materials design, adding additives into thermoplastic matrix that have yielded improvements. The effect of embedding short glass and carbon fibers in Acrylonitrile Butadiene Styrene (ABS) polymers was studied in terms of processability, microstructure and mechanical performance by Zhong et al. ${ }^{14}$. Wang et al. added thermally expandable microspheres into the matrix polymer and combined FDM process with thermal post-treatment to improve tensile strength. Sweeney et al. ${ }^{15}$ developed a concept for welding FDM thermoplastic interfaces using intense localized heating of carbon nanotubes (CNTs) by microwave irradiation. The CNTs were spatially localized at the interface by using a modified nozzle setup. They reported improvements in fracture strength by up to $275 \%$. It is interesting to note that some of these studies report improvements in mechanical performance along filament direction and not along the build $(z)$ direction. In addition, the improvements are attributed to the addition of a reinforcing phase or modifications during processing. Here, reinforcement of the weld interfaces is extrinsic in nature.

For poly(lactides) or PLA, one answer to improving the mechanical performance of the weld interface without the addition of extrinsic reinforcing phases may lie in controlling the spatial control of crystallization, particularly around the weld interface. Therefore, it is beneficial to look into the crystallization behaviour of PLA. Three isomeric forms of PLA exist according to the composition of enantiomers, i.e., poly (L-lactide) (PLLA), poly ( $D$-lactide) (PDLA) and poly (DL-lactide) (PDLLA). Polymers of predominantly $L$ or $D$ enantiomers can crystallize depending on the degree of purity. Polymers with low enantiomeric purity are typically amorphous. Three crystal unit cells have been reported and the structure adopted is a direct result of the crystallization conditions ${ }^{24-26}$. These comprise the orthorhombic a phase with $10_{3}$ helical chain conformation, a trigonal $B$ and orthorhombic $y$ forms both marked by $3_{1}$ helical chain conformation. Additionally, blending PLLA and PDLA results in stereocomplex or racemic crystallite formation. The packing density and thus spatial proximity of enantiomerically opposite chains increases, leading to energetically stronger intermolecular forces ${ }^{27-29}$. Stereocrystals possess a melting temperature of $220-230{ }^{\circ} \mathrm{C}$, which is $50{ }^{\circ} \mathrm{C}$ higher than that of PLLA or PDLA homocrystals $\left(170-180{ }^{\circ} \mathrm{C}\right)$. They also possess better mechanical properties, hydrolytic and thermal degradation-resistance, and gas barrier properties ${ }^{28,29}$. Today, several 
routes exist to produce stereocrystals with solution casting and melt blending being the most common. Other routes such as solid state drawing ${ }^{30}$, compression ${ }^{31}$ and repeated casting ${ }^{32}$ have also been developed. However, all these methods lead to stereocrystals that are distributed evenly in the polymer matrix, possibly with preferred spatial orientation, but not with preferred directed spatial distribution.

In this chapter, a novel route for the preparation and spatially directing of stereocomplex crystals across weld interfaces and consequent mechanical strengthening of FDM parts is proposed. Previously, a similar methodology, introducing interfacial stereocomplexation in multi-melt multi-injection molding with improved impact toughness and tensile strength, was studied by Chen et al. ${ }^{33}$. Fundamental insight in the correlation of twin nozzle FDM heat management with the kinetics of interfacial PLA stereocomplexation and consequential mechanical reinforcement is gained by combining polarized optical microscopy, rheometry, differential scanning calorimetry, spatially resolved Fourier transform infrared spectroscopy, synchrotron wide angle X-ray diffraction computed tomography, and dynamic mechanical thermal analysis.

\subsection{MATERIALS AND METHODS}

\subsubsection{Poly(lactides)}

Three commercial poly(L-lactide) (PLLA) grades with weight average molar masses $(M w)$ of 148 , 190 and $245 \mathrm{~kg} / \mathrm{mol}$, and a poly(D-lactide) (PDLA) grade of $100 \mathrm{~kg} / \mathrm{mol}$ weight average molar mass were provided by Corbion Purac. All grades have purity in excess of $99 \%$. Sample coding is in analogy to the molar mass, translating into PLLA148, PLLA190 and PLLA245 and PDLA100. Details of molecular weights and polydispersity (PDI) are provided in Table 4.1.

Table 4. 1: Number average molecular weight $\left(M_{n}\right)$, weight average molecular weight $\left(M_{w}\right)$ and Polydispersity (PDI) for PLLA and PDLA grades*

\begin{tabular}{|c|c|c|c|}
\hline Material & $M_{\mathrm{n}}(\mathbf{k g} / \mathrm{mol})$ & $M_{\mathrm{w}}(\mathbf{k g} / \mathrm{mol})$ & PDI \\
\hline PLLA245 & 117 & 245 & 2.1 \\
\hline PLLA190 & 90.5 & 190 & 2.1 \\
\hline PLLA148 & 72 & 148 & 2.05 \\
\hline PDLA100 & 47.5 & 100 & 2.1 \\
\hline
\end{tabular}

*Determined by Gel Permeation Chromatography (GPC) using HFIP as the eluent and relative to Poly(MethylMethAcrylate) (PMMA) $100000 \mathrm{~g} / \mathrm{mol}$ standards. 


\subsubsection{Kinetics and stiffness of stereocomplex formation at melt interface}

Stereocomplex formation at the interface of a PLLA- PDLA layer in the melt state was probed via rheometry. The materials were compression molded into discs of $25 \mathrm{~mm}$ diameter and 250 $\mu \mathrm{m}$ thickness using a mold temperature of $200{ }^{\circ} \mathrm{C}$. The samples were slowly cooled in order to attain maximum crystallinity. Thereafter, one PLLA and one PDLA disc were stacked, precompacted at low temperature and placed in a $25 \mathrm{~mm}$ diameter parallel plate geometry of a TA instruments DHR 1 rheometer. The system was heated at a rate of $10{ }^{\circ} \mathrm{C} / \mathrm{min}$ from room temperature to $200{ }^{\circ} \mathrm{C}$ and then kept isothermal for 1 hour, while employing an oscillatory shear of $0.1 \%$ and a frequency of $1 \mathrm{rad} / \mathrm{sec}$.

A temperature of $200{ }^{\circ} \mathrm{C}$ was chosen for the isothermal measurement. The temperature must be well above the melting temperature range of PLA homocrystals (upto $180^{\circ} \mathrm{C}$ as confirmed by Figure 4.1) but below the melting temperature of the PLA stereocrystals, typically in the range of $220-230{ }^{\circ} \mathrm{C}$. During the isothermal measurement, the storage modulus ( $G$ ') was measured as a function of time. A similar approach was adopted by Saeidlou et al. to investigate the kinetics of stereocomplex formation under isothermal conditions for melt blended PLLA and PDLA ${ }^{34}$.

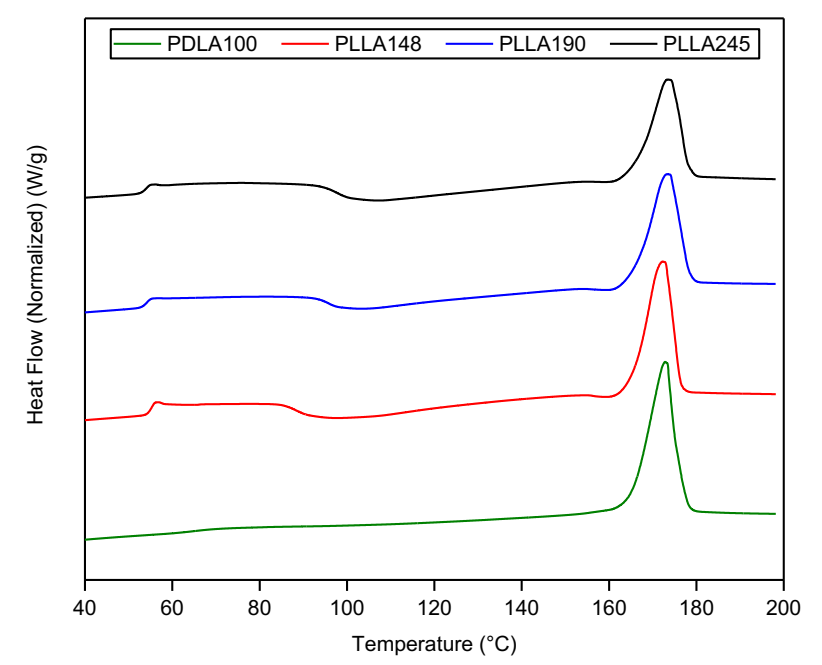

Figure 4.1: DSC thermograms for PLLA245, PLLA190, PLLA148 and PDLA100 on heating at $10^{\circ} \mathrm{C} / \mathrm{min}$ from room temperature to $200^{\circ} \mathrm{C}$.

After the measurement, the sample was cooled down to room temperature and extracted for Wide Angle $X$ ray Diffraction Computed Tomography (WAXDCT) measurements at the materials chemistry and materials engineering Beamline ID15A of the European Synchrotron Radiation Facility (ESRF), located in Grenoble, France. The incident beam had an energy of $65.351 \mathrm{keV}$, focused with lenses to an area of $\sim 20 \times 50 \mu \mathrm{m}^{2}(\mathrm{~V} \times \mathrm{H})$. Measurements were carried out with a -64 - 
resolution of $20 \times 100 \times 100 \mu \mathrm{m}^{3}(\mathrm{~V} \times \mathrm{H} \times \mathrm{H}) .46$ Layers were measured vertically, and $91 \times 101$ projections were taken in the plane in order to cover the entire sample and have a background in all dimensions for proper scaling. Count time was $50 \mathrm{~ms} /$ projection with 2D data collected up to a range of $5 \AA^{-1}$ with the Pilatus CdTe 2M detector. 2D patterns were corrected for geometry, detector distortion, response, and transparency. Furthermore, the 1D patterns were outiler-filtered and azimuthally integrated with a locally modified version of PyFAl ${ }^{35}$. The tomographic construction was made by performing filtered back-projection point by point on the sonograms of each layer constructed from the 3800 projections. After rearrangement, the result is a $3 \mathrm{D}$ matrix for each layer of the sample, with each $(x, y)$ voxel containing a reconstructed diffraction pattern from that voxel. These diffraction patterns can be analyzed to give spatially resolved crystal information about the sample in 3D. After background subtraction, the diffraction patterns were integrated over the homo- and stereocrystal peaks to generate tomographic reconstructs that reveal their 3D spatial phase distribution. Note that as the two phases have essentially identical density, it impossible to obtain a map from absorption or even phase-contrast tomography.

\subsubsection{Sample preparation via FDM}

Upstanding bars with a uni-directional $0^{\circ}$ deposition strategy were fabricated using an Arburg Freeformer. Sample geometry is illustrated in Figure 4.2. The parts consisted of alternating layers of PLLA and PDLA, for which the two nozzles were used in conjunction during the printing. Via a pressurized melt reservoir, stringed droplets were deposited through a nozzle with a high frequency piezo actuator. Sample bars were printed under optimized printing conditions that comprise a nozzle temperature of $200{ }^{\circ} \mathrm{C}$ and an ambient air temperature of $70{ }^{\circ} \mathrm{C}$. Additionally, as the cooling conditions influence the interlayer diffusion and potentially subsequent stereocomplex formation, three print speeds were chosen to range from low to high: 10, 20 and $50 \mathrm{~mm} / \mathrm{s}$.

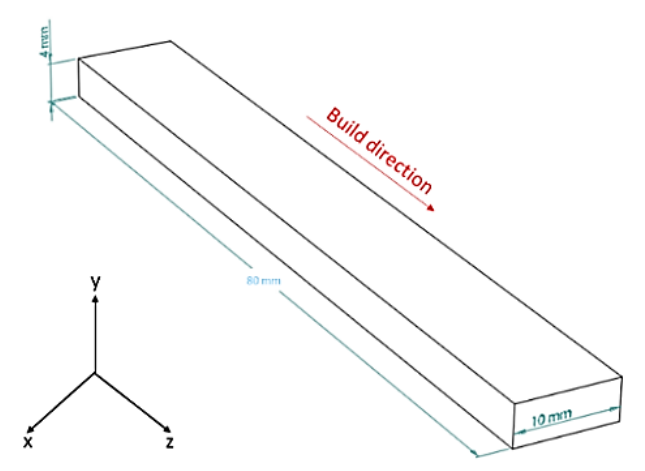

Figure 4.2: Schematic of sample geometry. 


\subsubsection{Characterization techniques for FDM samples}

To test the interlayer bonding mechanically, dynamic mechanical testing under torsion was carried out in an Anton Paar MCR 702 rheometer. The strain was set at $0.1 \%$, the frequency at $1 \mathrm{~Hz}$, and the storage modulus ( $\left.\mathrm{G}^{\prime}\right)$ was recorded in a temperature range from 30 and $120{ }^{\circ} \mathrm{C}$ using a heating rate of $5{ }^{\circ} \mathrm{C} / \mathrm{min}$. To understand the crystalline morphology across the layers, samples were cut into parts with a width of $3 \mathrm{~mm}$ from the top end of the sample. By cutting at the central region of the parts along the z-axis with a Leica EM UC7 ultra-microtome, thin slices of $2.5 \mu \mathrm{m}$ thickness were obtained. Polarization Optical Micrographs (POM) of the slices were taken between cross-polarizers on an Olympus BX53 microscope mounted with an Olympus DP26 camera. Fourier Transform Infrared (FTIR) spectra were recorded in transmission mode on a Perkin Elmer Spotlight $400 \mathrm{FT}(\mathrm{N}) \mathrm{IR}$ microscope. An aperture of $3 \times 150 \mu \mathrm{m}^{2}$, which was longitudinally positioned parallel to the interface, was used for spatially scanning the interfacial region, accumulating 256 spectra in the range of 4000 to $650 \mathrm{~cm}^{-1}$ with $1 \mathrm{~cm}^{-1}$ spectral resolution.

\subsection{RESULTS AND DISCUSSION}

\subsubsection{Isothermal interfacial stereocomplexation}

The mechanical response of stereocomplex formation at the interface of molten PLLA and PDLA was observed utilizing oscillatory shear rheometry at $200^{\circ} \mathrm{C}$. To follow the evolution of the storage modulus $G$ ' as a function of time the frequency was set at $1 \mathrm{rad} / \mathrm{s}$, representing zeroshear viscosity conditions for PLAs, Figure 4.3. Under these conditions, the behaviour as recorded by rheometry is not biased by shear. For all measurements, layer 1 is PDLA100 and layer 2 is made of PLLA with varying molar mass to influence molecular diffusion across the interface that precedes stereocrystallization. Figure 4.4 depicts the storage modulus $G$ ' of the molten enantiomerically opposite PLAs as a function of time at $200^{\circ} \mathrm{C}$. After 1 hour, all samples show an increase in $G^{\prime}$. Taking the melting temperatures of stereocrystals $\left(220-230{ }^{\circ} \mathrm{C}\right)$ into account, the increase in $G^{\prime}$ is likely due to the formation of a physical network in which the stereocrystals cooperatively provide resistance against shear deformation. To confirm that the observed increase in $G^{\prime}$ is due to the formation of stereocrystals, the same experiment is carried out by replacing PDLA100 with a PLLA grade with the same molar mass, named as PLLA100. The trends are given in open symbols in Figure 4.4 and confirm that the increase in G' in the absence of stereocrystals is negligible.

Though typical profiles for polymer crystallization over time are recognized, distinct differences for the different combinations of relative molar masses (or molar mass ratios) are observed in Figure 4.4. For the largest relative molar mass, i.e. PLLA245-PDLA100, the onset of crystallization is observed at about 400 seconds after which the storage modulus increases. -66 - 
Although the increase in storage modulus diminishes, not reaching a plateau indicates that stereocrystallization still occurs at the end of the experiment. For the lower relative molar mass, i.e. PLLA190-PDLA100 and PLLA148-PDLA100, stereocrystals are already being formed at the start of the isothermal measurement, time $t_{0}$. In fact, $G$ ' of the PLLA148-PDLA100 combination is higher than for PLLA 190-PDLA100 at $t_{0}$, which indicates that the extent of interfacial stereocrystallization at $t_{0}$ is the highest for the lowest relative molar mass. It is known that the overall crystallization rate of PLAs, in particularly this molar mass range, is affected by molar mass ${ }^{16,36,37}$. However, the process of stereocrystallization under these specific conditions does not classify as traditional crystal growth of polymers in bulk, where a polymer chain is reeled into the crystal growth front ${ }^{38}$.
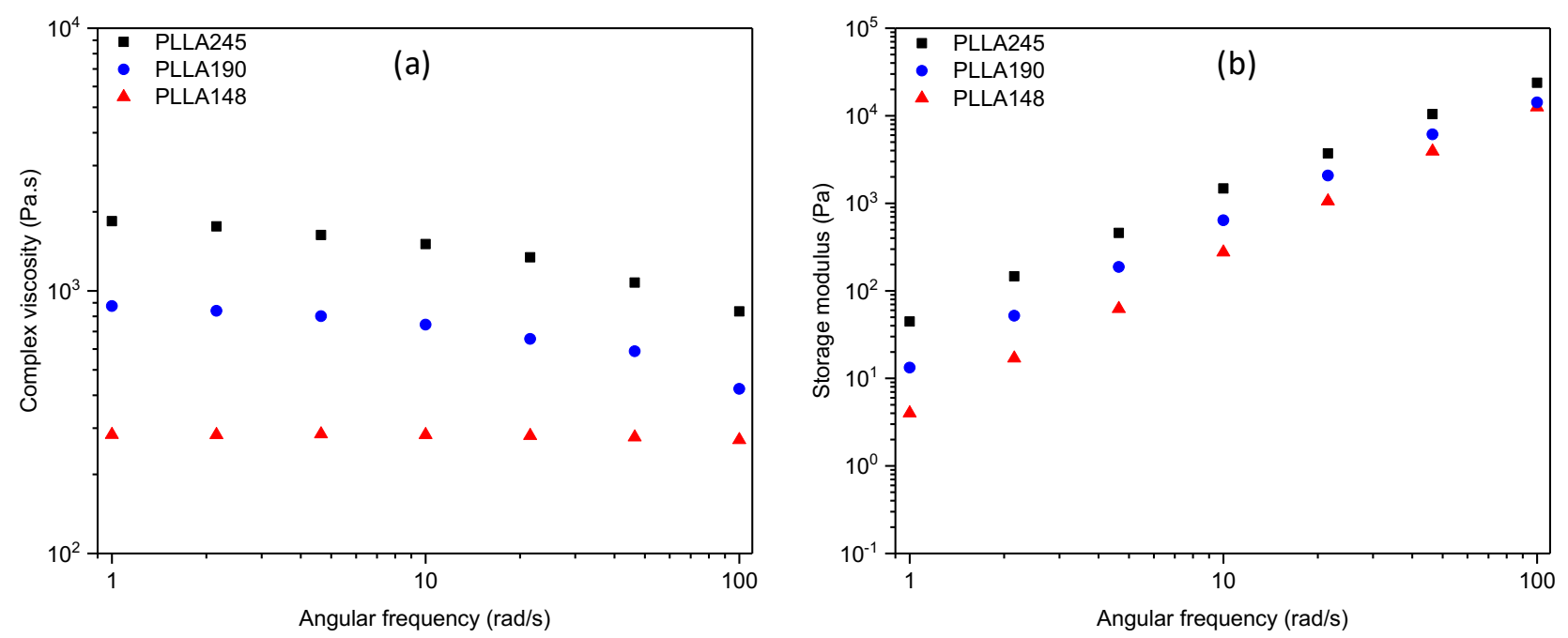

Figure 4.3: Complex viscosity (a) and storage modulus (b) of PLLA245, PLLA190 and PLLA148 plotted as a function of angular frequency.

The dynamics of interfacial stereocomplexation as registered by rheometry depend on molecular diffusion of one stereo-specific PLA into the other and vice-versa - the process that precedes crystallization. The diffusivity $(D)$ of polymers is inversely related to the molar mass (M) by $D \propto M^{-2}$ as described by the reptation theorem ${ }^{39}$. As the molar mass of the PDLA is equal in all three experiments, the increasing molar mass of PLLAs seems to govern the rate of stereocrystallization. Moreover, as time progresses and the rate of stereocomplex formation decreases, the highest interfacial stiffness is observed for PLLA245-PDLA100. It is likely that due to the higher molar mass of PLLA245 the physical network formed is denser and mechanically more resilient. Similar results were reported by Wei et al. ${ }^{40}$, who reported that stereocrystals in highly asymmetric PLLA-PDLA blends stiffen the PLLA melt due to a physical cross-linking effect. Albeit the fact that the highest ultimate $G$ ' is witnessed for the highest relative molar 
mass, missing the starting of G' of the lower relative molar mass samples hinders insight in the absolute stiffening of the interface.

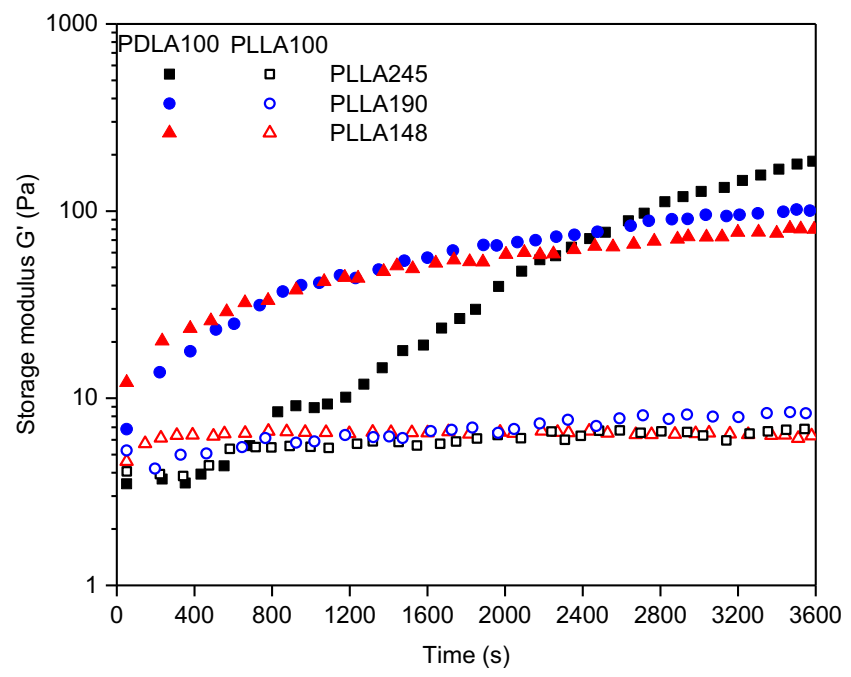

Figure 4.4: Storage Modulus (G') at $1 \mathrm{rad} / \mathrm{s}$ of two pre-compacted molten PLA disks with opposite (closed symbols) and identical (open symbols) enantiomeric composition as a function of time to probe the kinetics of interfacial stereo complexation upon change in relative molar mass at $200{ }^{\circ} \mathrm{C}$.

Further insight into the spatial distribution of stereocrystals in the cooled rheometry discs is provided via synchrotron Wide Angle X-ray Diffraction Computed Tomography (WAXDCT). The tomographic reconstruction of the diffraction patterns, presented in Figure 4.5, is based on the $(110) /(200)$ and (110) diffraction signals of the homo and stereocrystals respectively as highlighted in Figure 4.5(a). Note that cooling of the rheometry discs at the end of the experiment induces additional homocrystallization. The primary crystalline phase is associated with PLLA and PDLA homocrystals with different diffraction peaks at $q$ values of 1.17 and 1.34 $A^{-1}$. The second crystalline phase is of the stereocrystals and is represented by diffraction signals at $q$ values of 0.84 and $1.48 \mathrm{~A}^{-1}$. 

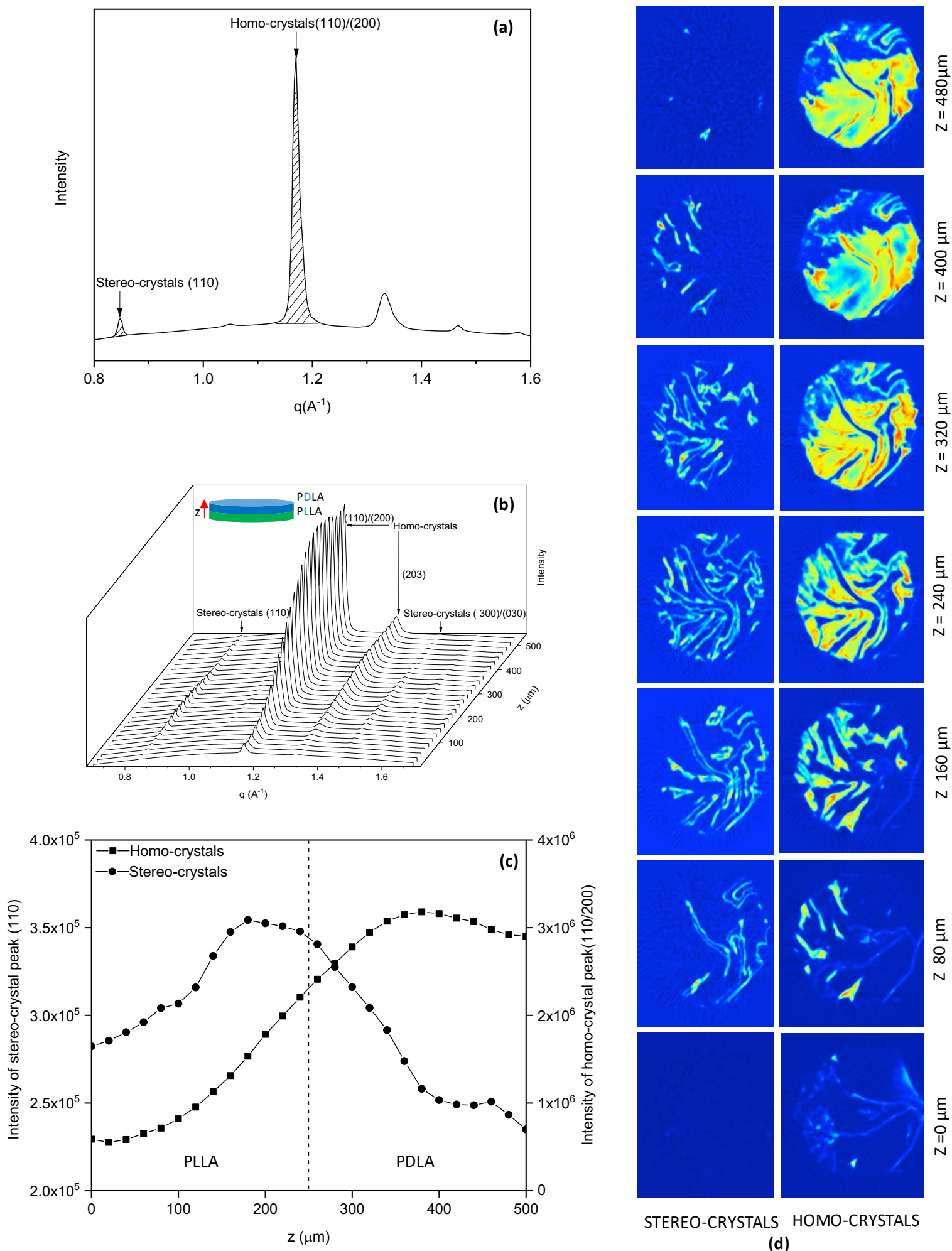

STEREO-CRYSTALS HOMO-CRYSTALS

(d)

Figure 4.5: Tomographic reconstruction of a PLLA148-PDLA100 rheometry disk based on wide angle Xray diffraction, visualizing the spatial distribution of a homo- and stereocrystals. The reconstruction is based on the integration of the (110)/ (200) and (110) diffraction signals of the homo- and stereocrystals, as indicated in (a), respectively. Layer (z) averaged integrated diffraction patterns as a function of the $z$-axis are plotted in (b), whereas the spatial distribution in $3 D$ is given in (d). The averaged population of homo- and stereocrystals as a function of sample thickness is depicted by the intensity of the (110)/(200) and (110) as a function of z respectively, (c). (Red = high intensity, Blue = low intensity). 
Tomographic reconstructions revealing the 3D spatial distribution of stereo- and homocrystals in different $x-y$ planes across the sample thickness ( $z$ ) are shown in Figure 4.5(d) and complimented by averaged integrated diffraction patterns as a function of sample thickness in Figure 4.5(b). The intensity of the representative stereo- and homocrystal diffraction peak, and thus their proportional fractions, are plotted in Figure 4.5(c). The initial z-value of the interface is at $250 \mu \mathrm{m}$, but it is evident that the presence of stereocrystals concentrated in the z-region from about 80 to $330 \mu \mathrm{m}$ with a distinctly higher fraction on the initial PLLA side of the interface. Within the timescales of the experiment, it is predominantly the relatively low molar mass PDLA that diffuses into the PLLAs, featuring lower diffusivity and higher zero-shear viscosity with increasing molar mass. Hence, by means of the different relative molar mass of the enantiomerically opposite PLAs the spatial size and mechanical significance of the interface in FDM can be tailored. Figure 4.5(c) also reveals that upon cooling the sample with $10{ }^{\circ} \mathrm{C} / \mathrm{min}$ homocrystallization is observed for the PDLA100, typically above a z-value above $330 \mu \mathrm{m}$. Descending through the sample, i.e. reducing $z$, the fraction of homocrystals tends to increase initially before it decreases. The comparison of the tomographic intensity plots of the homoand stereocrystals in the z-range of 160 to $320 \mu \mathrm{m}$ reveals that the plots are mirror images. Stereocrystallization thus occurs in spatially small areas where, upon cooling, no homocrystallization occurs. This explains the decreased fraction of PDLA100 homocrystals in the mixing zone. Due to the increased molar mass, no or marginal homocrystallization of the PLLA148 is observed at low z-values. However, upon increasing the z-value, the fraction of homocrystals increases that taking the increased enthalpy of stereocrystals into account ${ }^{28,29,36}$, is unlikely to originate in homocrystallization of PDLA100. Instead, stereocrystals formed at 200 ${ }^{\circ} \mathrm{C}$ seem to promote homocrystallization of PLLA148 upon cooling at $10{ }^{\circ} \mathrm{C} / \mathrm{min}$ via enhanced nucleation $18{ }^{40}$. The relatively low homocrystallization rates of the pure enantiomerically opposite PLAs and successive nucleation effects of the stereocrystals seem ideal to solve the compromise between interfacial bonding and complete crystallization securing thermodynamic stability in FDM ${ }^{16}$. It must be kept in mind that translating this concept to FDM imposes additional challenges. FDM processes involve complicated sequential heating and cooling steps that induce re-melting, cold and melt crystallization, and annealing. The timescale for interfacial diffusion and successive stereocrystallization in FDM is limited. Mcllory et al. ${ }^{41}$ predicted inter-diffusion between layers to occur over length-scales matching the radius of gyration. In the next section, a translation of the proposed concept to the non-isothermal conditions of a twin headed FDM process will be elaborated. 


\subsubsection{Non-isothermal interfacial stereocomplexation in FDM printing}

The effect of interfacial stereocomplexation on (interfacial) structure evolution and stiffness in FDM was studied via alternating deposition of PLLA and PDLA layers. Since the highest rate of stereocrystallization under isothermal conditions was observed for the lowest relative molar mass, all parts were printed using the combination PLLA148-PDLA100. Furthermore, local heat dosage to direct the extent of molecular diffusivity across the interface was varied by three different print speeds, namely 10, 20 and $50 \mathrm{~mm} / \mathrm{s}$. By means of an ultra-microtome, thin slices of the core of the sample were cut parallel to the build $(z)$ direction. Figure 4.6 displays the crossed polarized optical micrographs.

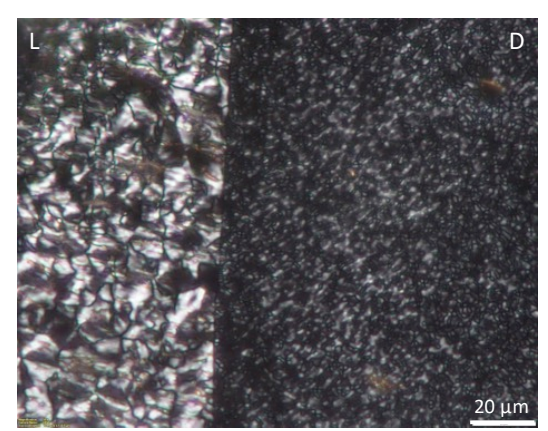

(a)

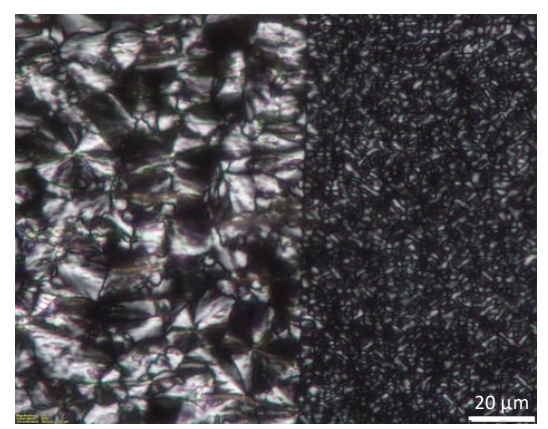

(b)

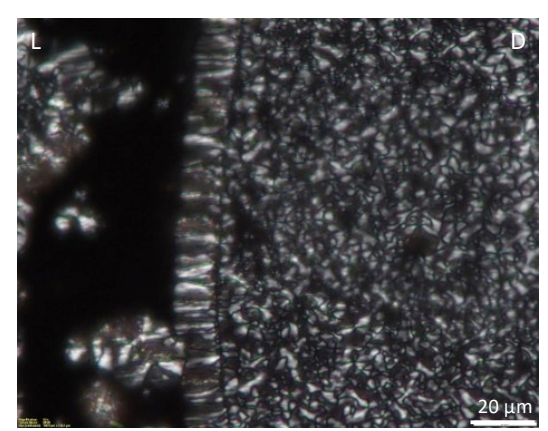

(c)

Figure 4.6: Polarized optical micrographs of printed interfaces with left PLLA and right PDLA layers at the print speeds (a) $10 \mathrm{~mm} / \mathrm{s}$, (b) $20 \mathrm{~mm} / \mathrm{s}$ and (c) $50 \mathrm{~mm} / \mathrm{s}$.

For all print speeds, the interface is clearly recognizable as the homocrystals of PLLA and PDLA on either side of the interface differ in size due to the different crystallization dynamics ${ }^{16,36}$. Whereas the bulk filament of the PDLA shows high crystallinity at all printing speeds, the molecular mass induced relatively low crystallization rate of PLLA148 suppresses high crystallinity at a print speed of $50 \mathrm{~mm} / \mathrm{s}$. From our previous studies, it is known that despite less efficient heat dissipation at high print speeds, the average annealing temperature and time upon deposition of new layers decreases. This, in turn, promotes incomplete crystallization and thus thermodynamic and geometrical instability ${ }^{16}$. 


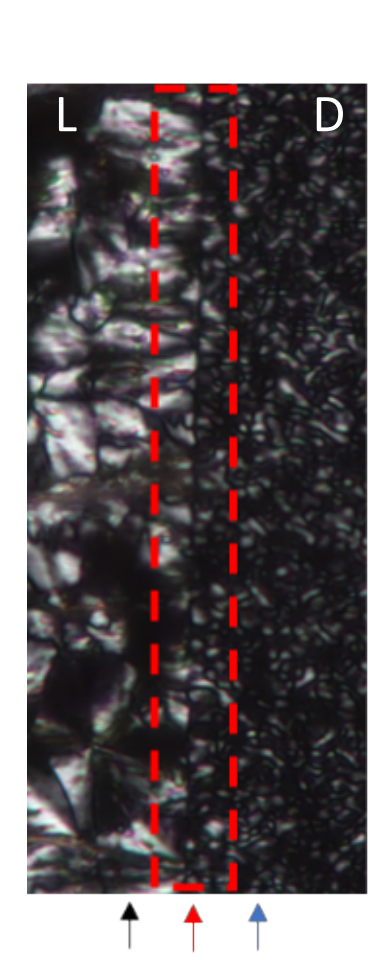

(a)

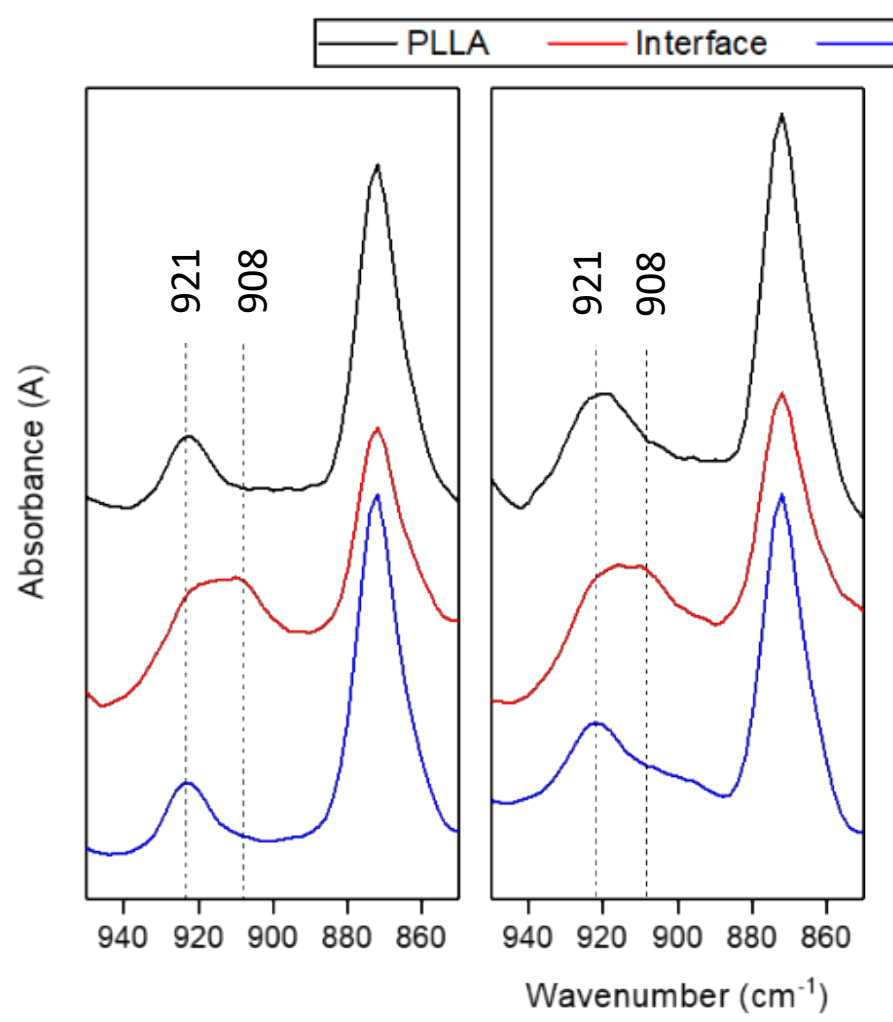

(c)

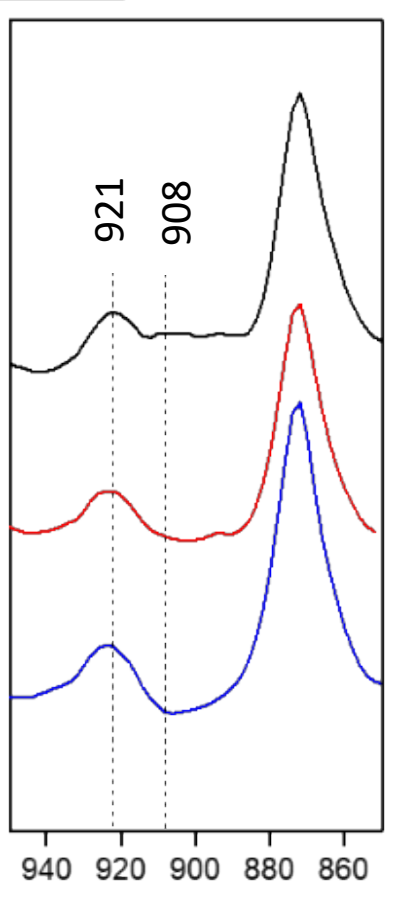

(d)

Figure 4.7: Spatially resolved FTIR spectra recorded at (red), left from (black) and right from (blue) the interface as depicted in figure (a). The effect of print speed, being (b) $10 \mathrm{~mm} / \mathrm{s}$, (c) $20 \mathrm{~mm} / \mathrm{s}$, and (d) $50 \mathrm{~mm} / \mathrm{s}$, on the structure at and on either side of the interface is highlighted by the spectral changes.

The optical micrograph of Figure 4.6(c) moreover reveals an anisotropic crystallization layer that extends from the interface into the PLLA bulk. Are these stereo- or homocrystals? And in the case of homocrystals, are they cold crystallized upon reheating or nucleated from homo PDLA or stereocrystals at the interface? To answer these questions spatially resolved FTIR spectra were recorded on both semi-crystalline sides of, and at the interface using an aperture of $3 \times 150 \mu \mathrm{m}^{2}$ that was longitudinally aligned parallel to the interface as seen in Figure 4.7. The PLA chain conformation in the stereocrystals results in a unique vibrational band at $908 \mathrm{~cm}^{\text {- }}$ 142,43 . The band at $921 \mathrm{~cm}^{-1}$, present in all three regions, is assigned to the coupling of C-C backbone stretching with the $\mathrm{CH}_{3}$ rocking mode and is representative of the $10_{3}$ helical chain conformation of a homocrystals ${ }^{42}$. To verify the band assignment for the selected PLLA and PDLA grades FTIR spectra of samples purely consisting of stereo- or homocrystals were recorded as a reference and embedded in Figure C1 of Appendix C. The FTIR spectra reveal that like in the ultimate rheometry discs the mixing zone consists of local regions of either stereo- or homocrystallites. The ratio of 908 and $921 \mathrm{~cm}^{-1}$ peaks serves as a relative measure of the amount of stereocrystals at the interface that decreases with increased print speed. At a speed of 50 
$\mathrm{mm} / \mathrm{s}$ the vibrational band at $908 \mathrm{~cm}^{-1}$ is not observed, which suggests the absence or a nondetectable amount of stereocrystals. It is the net total spatial heat dosage at the moment of melt deposition that defines the length-scales of interfacial molecular diffusion, and thus stereocrystallization. At high print speed, the heated nozzle only slightly reheats a filament from a previously deposited layer, limiting molecular diffusion and mechanically resilient weld formation. Depending on the crystallization rate, reheating by a newly deposited layer may induce cold crystallization. If the melting temperature upon reheating is not reached, molecular diffusion and interfacial stereocomplexation will be hindered even more. The layer of anisotropically grown crystals in the PLLA phase printed at a speed of $50 \mathrm{~mm} / \mathrm{s}$ consists of a homocrystals. Remarkably, such a nucleated layer of anisotropic PLLA crystals in spatial proximity of the interface is barely observed at lower print speeds though the net local heat dosage and so the potential for stereocrystallization increases. Since the $908: 921 \mathrm{~cm}^{-1}$ ratio increases with decreasing print speed and the stereocrystal representative $908 \mathrm{~cm}^{-1}$ band is absent at a print speed of $50 \mathrm{~mm} / \mathrm{s}$, the PLLA homocrystals likely transcrystallized directly from the semi-crystalline PDLA front. These findings are in correspondence to the work of Wen et al. who reported that in transcrystallization of PLAs the $3_{1}$ helical chain conformation of stereocomplexed chains possess an epitaxial mismatch to the $10_{3}$ helical chain conformation adopted in a homocrystals ${ }^{44}$. Although transcrystallization occurs from stereocomplex as well as from orthorhombic a crystal interfaces, the nucleation efficiency of stereocrystals is relatively low. The effect of interfacial stereocrystallization and/or PDLA induced transcrystallization on the mechanical reinforcement of the weld-interface was studied by means of torsional Dynamic Mechanical Analysis (DMA).

\subsubsection{Mechanical properties of 3D printed parts}

The crystalline morphology plays an important role in the thermomechanical performance of a semi-crystalline polymer. The PLLA-PDLA structural morphology created by the twin print setup consists of mainly of homocrystals and small regions of stereocrystals across the weld interfaces. In torsional oscillatory DMA, stresses are specifically transferred to the $x-y$ planes of the FDM parts and thus the weld regions. Three sample categories are defined. Category (A) comprises annealed injection molded PLA bars with maximum crystallinity, representing the intrinsic bulk stiffness of the PLAs. Categories (B) and $(C)$ are marked by FDM samples with and without stereocomplexation respectively. Although printed under identical conditions, the samples of category (B) were based on alternating PLLA148 and PDLA100 layers, while samples of category (C) are made of PLLA148 or PDLA100 layers only. The DMA results of all samples are given in Figure 4.8. Only the samples printed at $50 \mathrm{~mm} / \mathrm{s}$ show a slight recovery/increase of the storage modulus due to cold crystallization above $T_{\mathrm{g}}$ (except PDLA100). This means that with the exception of the $50 \mathrm{~mm} / \mathrm{s}$ printed samples, all samples have attained maximum crystallinity. 

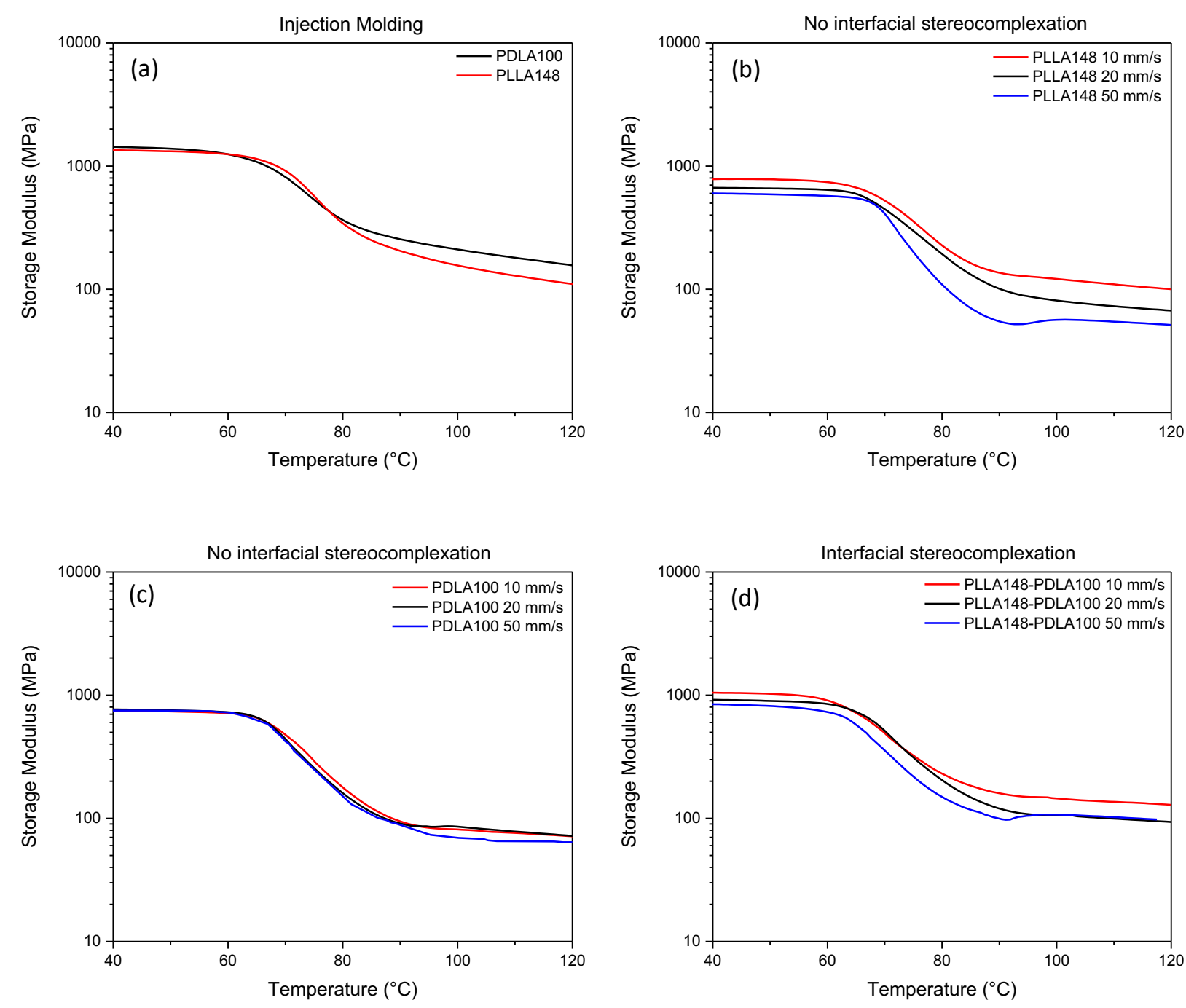

Figure 4.8: Storage modulus as a function of temperature for samples obtained via injection molding (a), samples with no interfacial stereocomplexation (b) and (c), samples with interfacial stereocomplexation $(d)$.

Analyzing the storage modulus of the printed samples below $T_{g}$, the effect of increased net local heat dosage on mechanical stiffening of the weld interfaces via enhanced diffusivity is evident (Figure 4.9). On comparing samples printed without stereocomplexation (PLLA148 and PDLA100 in Category C), lowering the molar mass leads to slight increase in storage modulus at all print speeds due to higher diffusivity and consequently higher interpenetration depth at the interface.More importantly, independent of the print speed printing alternating layers of enantiomerically opposite PLAs leads to an increase in storage modulus of approximately $40 \%$. From these results can be concluded that both interfacial stereocrystallization, as well as direct transcrystallization from the first homocrystallizing front, induce substantial reinforcement of fused deposition modeled parts in the z-direction. 


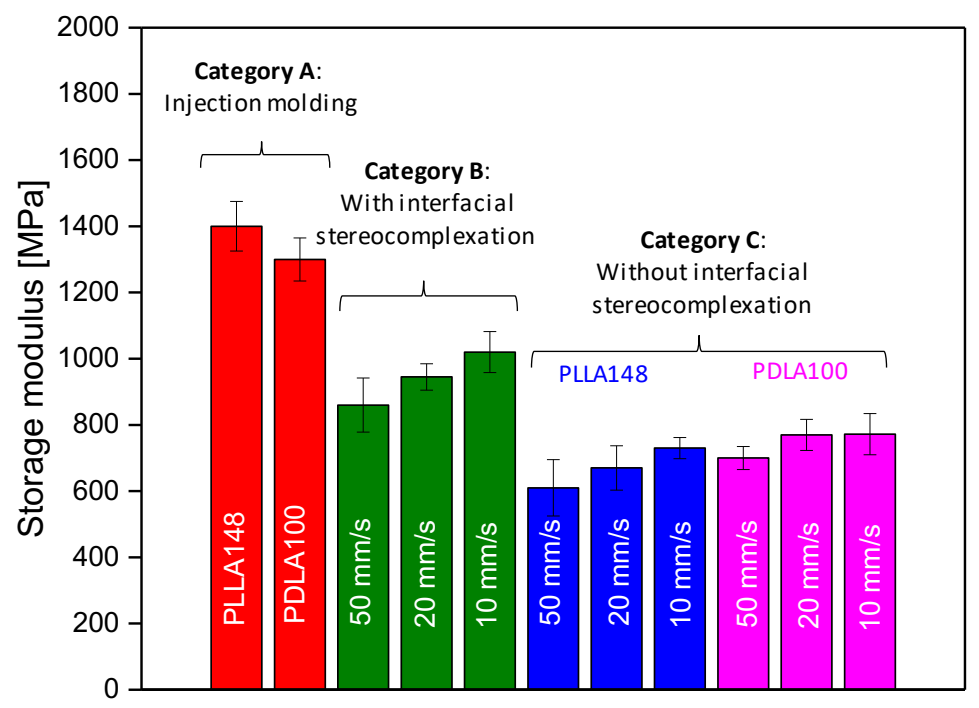

Figure 4.9: Storage modulus at $40^{\circ} \mathrm{C}$ for categories $A, B$ and $C$. Category $A$ represents bulk stiffness of the materials obtained via injection molding. Category $B$ and $C$ represent interlayer stiffness obtained via FDM with and without the presence of stereocomplexation respectively.

\subsection{CONCLUSIONS}

Stereocomplexation can be spatially directed to melt interfaces of enantiomerically opposite polymers. Following the evolution of the storage modulus upon isothermal stereocomplexation of poly(L-lactide) and poly(D-lactide) across melt interfaces between the melting temperatures of the homo- and stereocrystals, reveals that the rate of interfacial stereocomplexation depends on the absolute and relative molar masses. A low relative molar mass results in a high rate of interfacial stereocomplexation, but relatively low ultimate storage modulus and thus interfacial stiffening. In contrast, the decreased diffusivity for high relative molar masses induces slow stereocomplexation, but a stiff final physical network. By means of micro-focus wide angle Xray diffraction computed tomography the $3 \mathrm{D}$ spatial distribution of stereocrystals across the interface can be reconstructed. Inter-diffusion of predominantly the low molar mass into the high molar mass phase stiffens the interface and promotes homocrystallization upon cooling. Thus, by a judicious selection of absolute and relative molar masses of two enantiomerically opposite poly(lactides), the length-scales and mechanical reinforcement of stereocomplexed interfaces can be tailored.

Interfacial stereocomplex formation across fused deposition modeled weld interfaces was realized by depositing alternating layers of poly(D-lactide) and poly(L-lactides) using a twin nozzle setup and relative low molar masses. The extent and mechanical significance of interfacial stereocomplexation under the complex non-isothermal conditions depends on the 
print speed and the net local heat dosage. Low print speeds result in a higher concentration of stereocrystals as sufficient heat ensures (i) conservation of the melt state of a freshly deposited filament, (ii) reheating a previously deposited filament into its melt, and (iii) sufficient time for molecular diffusion to occur across the interface. All three are prerequisites to interfacial stereocomplexation during fused deposition modeling. Contrary, high print speeds suppress stereocomplexation and, dependent on relative molar mass, may promote strong interfacial transcrystallization of poly(L-lactides) if the crystallization rate of poly( $\mathrm{D}$-lactide) if cooling is sufficiently high. The interfacial nucleation effect induced by the poly(lactide) stereocrystals is less due to the epitaxial mismatch of stereo- and homocrystals. Nucleation of the bulk filaments after stereocomplexation induces stiffening and aids in the thermodynamic and geometrical stability of fused deposition modeled products. In conclusion, interfacial stereocomplexation and transcrystallization of enantiomerically opposite poly(lactides) is a simple and effective way to induce a substantial $40 \%$ increase in stiffness without the aid of extrinsic reinforcing components. 


\section{REFERENCES}

(1) Ligon, S. C.; Liska, R.; Stampfl, J.; Gurr, M.; Mülhaupt, R., Polymers for 3D Printing and Customized Additive Manufacturing. Chemical Reviews. 2017, 117 (15), 10212-10290.

(2) Wendel, B.; Rietzel, D.; Kühnlein, F.; Feulner, R.; Hülder, G.; Schmachtenberg, E., Additive Processing of Polymers. Macromolecular Material and Engineering. 2008, 293 (10), 799-809.

(3) Masood, S. H.; Song, W. Q. ,Development of New Metal/Polymer Materials for Rapid Tooling Using Fused Deposition Modelling. Materials and Design. 2004, 25 (7), 587-594.

(4) Kim, M.-S.; Chu, W.-S.; Kim, Y.-M.; Avila, A. P. G.; Ahn, S.-H., Direct Metal Printing of 3D Electrical Circuit Using Rapid Prototyping. International Journal of Precision Engineering and Manufacturing. 2009, 10 (5), 147-150.

(5) Braunschweig, A. B.; Huo, F.; Mirkin, C. A., Molecular Printing. Nat Chem 2009, 1 (5), 353-358.

(6) Norotte, C.; Marga, F. S.; Niklason, L. E.; Forgacs, G., Scaffold-Free Vascular Tissue Engineering Using Bioprinting. Biomaterials. 2009, 30 (30), 5910-5917.

(7) Gebhardt, A., Understanding Additive Manufacturing. 2011, I-IX, ISBN 9783446425521.

(8) Yin, J.; Lu, C.; Fu, J.; Huang, Y.; Zheng, Y., Interfacial Bonding during Multi-Material Fused Deposition Modeling (FDM) Process Due to Inter-Molecular Diffusion. Materials and Design. 2018, 150, 104-112.

(9) Ahn, S.; Montero, M.; Odell, D.; Roundy, S.; Wright, P. K. ,Anisotropic Material Properties of Fused Deposition Modeling ABS. Rapid Prototypyping Journal. 2002, 8 (4), 248-257.

(10) Wang, J.; Xie, H.; Weng, Z.; Senthil, T.; Wu, L. A Novel Approach to Improve Mechanical Properties of Parts Fabricated by Fused Deposition Modeling. Materials and Design. 2016, 105, 152-159.

(11) Lanzotti, A.; Grasso, M.; Staiano, G.; Martorelli, M., The Impact of Process Parameters on Mechanical Properties of Parts Fabricated in PLA with an Open-Source 3-D Printer. Rapid Prototyping Journal. 2015, 21 (5), 604-617.

(12) Agarwala, M. K.; Jamalabad, V. R.; Langrana, N. A.; Safari, A.; Whalen, P. J.; Danforth, S. C., Structural Quality of Parts Processed by Fused Deposition. Rapid Prototyping Journal. 1996, 2 (4), 4-19.

(13) Sood, A. K.; Ohdar, R. K.; Mahapatra, S. S., Parametric Appraisal of Mechanical Property of Fused Deposition Modelling Processed Parts. Materials and Design. 2010, 31 (1), 287-295.

(14) Zhong, W.; Li, F.; Zhang, Z.; Song, L.; Li, Z., Short Fiber Reinforced Composites for Fused Deposition Modeling. Materials Science and Engeering. A 2001, 301 (2), 125-130.

(15) Sweeney, C. B.; Lackey, B. A.; Pospisil, M. J.; Achee, T. C.; Hicks, V. K.; Moran, A. G.; Teipel, B. R.; Saed, M. A.; Green, M. J., Welding of 3D-Printed Carbon Nanotube-polymer Composites by Locally Induced Microwave Heating. Science Advances. 2017, 3 (6)

(16) Srinivas, V.; van Hooy-Corstjens, C. S. J.; Harings, J. A. W., Correlating Molecular and Crystallization Dynamics to Macroscopic Fusion and Thermodynamic Stability in Fused Deposition Modeling; a Model Study on Polylactides. Polymer. 2018, 142, 348-355.

(17) Levenhagen, N. P.; Dadmun, M. D., Bimodal Molecular Weight Samples Improve the Isotropy of 3D Printed Polymeric Samples. Polymer. 2017, 122 (Supplement C), 232-241..

(18) Levenhagen, N. P.; Dadmun, M. D. Interlayer Diffusion of Surface Segregating Additives to Improve the Isotropy of Fused Deposition Modeling Products. Polymer. 2018, 152, 35-41.

(19) Schach, R.; Creton, C., Adhesion at Interfaces between Highly Entangled Polymer Melts. Journal of Rheology . 2008, 52 (3), 749-767.

(20) Panda, B. N.; Shankhwar, K.; Garg, A.; Jian, Z., Performance Evaluation of Warping Characteristic of Fused Deposition Modelling Process. International Journal of Advanced Manufacturing Technology. 2017, 88 (5), 1799-1811.

(21) Alsoufi, M.; Abdulrehman, J., Warping Deformation of Desktop 3D Printed Parts Manufactured by Open Source Fused Deposition Modeling (FDM) System. International Journal of Mechanical and Mechatronics Engineering. 2017, 17 (4), 7-16.

(22) Seo, Y.; Kim, J.; Kim, K. U.; Kim, Y. C. Study of the Crystallization Behaviors of Polypropylene and Maleic Anhydride Grafted Polypropylene. Polymer. 2000, 41 (7), 2639-2646.

(23) Wang, L.; Gramlich, W.; Gardner, D.; Han, Y.; Tajvidi, M., Spray-Dried Cellulose NanofibrilReinforced Polypropylene Composites for Extrusion-Based Additive Manufacturing: Nonisothermal Crystallization Kinetics and Thermal Expansion. Journal of Composites Science. 2018, 2 (1), 7.

(24) Tsuji, H.; Takai, H.; Saha, S. K., Isothermal and Non-Isothermal Crystallization Behavior of Poly(lLactic Acid): Effects of Stereocomplex as Nucleating Agent. Polymer. 2006, 47 (11), 3826-3837.

(25) Yasuniwa, M.; Tsubakihara, S.; lura, K.; Ono, Y.; Dan, Y.; Takahashi, K., Crystallization Behavior of Poly(l-Lactic Acid). Polymer. 2006, 47 (21), 7554-7563.

(26) Kawai, T.; Rahman, N.; Matsuba, G.; Nishida, K.; Kanaya, T.; Nakano, M.; Okamoto, H.; Kawada, J.; Usuki, A.; Honma, N.; et al., Crystallization and Melting Behavior of Poly (l-Lactic Acid). 
Macromolecules. 2007, 40 (26), 9463-9469.

(27) Tsuji, H.; Ikada, Y., Stereocomplex Formation between Enantiomeric Poly(Lactic Acid)s. XI. Mechanical Properties and Morphology of Solution-Cast Films. Polymer. 1999, 40 (24), 6699-6708.

(28) Tsuji, H., Poly(Lactide) Stereocomplexes: Formation, Structure, Properties, Degradation, and Applications. Macromolecular Bioscience. 2005, 5 (7), 569-597.

(29) Tsuji, H., Poly(Lactic Acid) Stereocomplexes: A Decade of Progress. Advances in Drug Delivery Reviews. 2016, 107, 97-135.

(30) Tsuji, H.; Ikada, Y.; Hyon, S.-H.; Kimura, Y.; Kitao, T., Stereocomplex Formation between Enantiomeric Poly(Lactic Acid). VIII. Complex Fibers Spun from Mixed Solution of Poly(D-Lactic Acid) and Poly(L-Lactic Acid). Journal of Appled Polymer Science. 1994, 51 (2), 337-344.

(31) Bao, R.-Y.; Yang, W.; Wei, X.-F.; Xie, B.-H.; Yang, M.-B., Enhanced Formation of Stereocomplex Crystallites of High Molecular Weight Poly( l -Lactide)/Poly( d -Lactide) Blends from Melt by Using Poly(Ethylene Glycol). ACS Sustainal Chemistry and Engineering. 2014, 2 (10), 2301-2309.

(32) Hideto, T.; Satomi, Y., Enhanced Stereocomplex Crystallization of Biodegradable Enantiomeric Poly(Lactic Acid)s by Repeated Casting. Macromolecular Materials Engineering. 2011, 296 (7), 583589.

(33) Chen, R.; Huang, Y.-H.; Liu, L.; Chen, L.-B.; Liu, Z.-Y.; Yang, W.; Yang, M.-B., Diverse Interfacial Crystalline Morphologies Induced by Poly (d-Lactide) (PDLA) Melt Penetration Process in Multi-Melt Multi-Injection Molding (M3IM) System. Composites Part B: Engineering. 2018, 153, 429-436.

(34) Saeidlou, S.; Huneault, M. A.; Li, H.; Park, C. B., Poly(Lactic Acid) Stereocomplex Formation: Application to PLA Rheological Property Modification. Journal of Applied Polymer Science. 2014, $131(22)$

(35) Kieffer, J.; Petitdemange, S.; Vincent, T., Real-Time Diffraction Computed Tomography Data Reduction. Journal of Synchrotron Radiation. 2018, 25, 612-617.

(36) Saeidlou, S.; Huneault, M. A.; Li, H.; Park, C. B., Poly(Lactic Acid) Crystallization. Progress in Polymer Science. 2012, 37 (12), 1657-1677.

(37) Nunes, R. W.; Martin, J. R.; Johnson, J. F., Influence of Molecular Weight and Molecular Weight Distribution on Mechanical Properties of Polymers. Polymer Engineering and Science. 1982, 22 (4), 205-228.

(38) Piorkowska, E.; Rutledge, G., Handbook of Polymer Crystallization; 2013, ISBN 9780470380239.

(39) Brochard, F.; Jouffroy, J.; Levinson, P., Polymer-Polymer Diffusion in Melts. Macromolecules. 1983, 16 (10), 1638-1641.

(40) Wei, X.-F.; Bao, R.-Y.; Cao, Z.-Q.; Yang, W.; Xie, B.-H.; Yang, M.-B., Stereocomplex Crystallite Network in Asymmetric PLLA/PDLA Blends: Formation, Structure, and Confining Effect on the Crystallization Rate of Homocrystallites. Macromolecules. 2014, 47 (4), 1439-1448.

(41) Mcllroy, C.; Olmsted, P. D., Disentanglement Effects on Welding Behaviour of Polymer Melts during the Fused-Filament-Fabrication Method for Additive Manufacturing. Polymer. 2017, 123, 376-391.

(42) Pan, P.; Yang, J.; Shan, G.; Bao, Y.; Weng, Z.; Cao, A.; Yazawa, K.; Inoue, Y., TemperatureVariable FTIR and Solid-State ${ }^{13} \mathrm{C}$ NMR Investigations on Crystalline Structure and Molecular Dynamics of Polymorphic Poly( I -Lactide) and Poly( l -Lactide)/Poly( d -Lactide) Stereocomplex. Macromolecules. 2012, 45 (1), 189-197.

(43) Zhang, J.; Tashiro, K.; Tsuji, H.; Domb, A. J., Disorder-to-Order Phase Transition and Multiple Melting Behavior of Poly(l-Lactide) Investigated by Simultaneous Measurements of WAXD and DSC. Macromolecules. 2008, 41 (4), 1352-1357.

(44) Wen, T.; Xiong, Z.; Liu, G.; Zhang, X.; de Vos, S.; Wang, R.; Joziasse, C. A. P.; Wang, F.; Wang, D., The Inexistence of Epitaxial Relationship between Stereocomplex and a Crystal of Poly(Lactic Acid): Direct Experimental Evidence. Polymer. 2013, 54 (7), 1923-192 
- 79. 


\section{Chapter 5}

\section{Time- and length-scales of interfacial stereocomplexation in heterogeneous poly(lactide) melts}

This chapter contains unpublished work submitted to Additive Manufacturing Journal. 


\section{ABSTRACT}

To understand the realization of effective stress transfer at polymer-polymer interfaces by the concept of interfacial stereocrystallization, the effect of temperature and molar mass ratio are studied in heterogeneous poly(lactide) melt-states. Whereas the stereocrystallization rate is dictated by supercooling and relative viscosities, the length-scales depend on the formation of stereocrystalline domains connected via amorphous regions resulting in network formation, gelation. Upon gelation, further crystallization is impeded, which is supported by rheometry, DSC and FTIR imaging. When the initial relative viscosity between the PLA fractions is low, the time to reach critical network density is high. Retrospectively, the length-scales of stereocrystallization and ultimate mechanical stiffening are strongly influenced by the chosen temperature and molar mass ratio of the PLA fractions. This fundamental understanding of the time- and length-scales of interfacial diffusion, successive stereocrystallization, and nucleation of homocrystals upon further cooling, assists in the technical realization of mechanically reinforced polymer-polymer interfaces. 


\subsection{INTRODUCTION}

Interfacial crystallization has attracted attention in the past decades, especially in the field of polymer composites. It has opened a promising pathway to enhance interfacial adhesion and consequential stress transfer between a polymeric matrix and filler, translating to enhanced mechanical properties. Studies on interfacial crystallization have revealed the existence of several semi-crystalline morphologies such as trans crystals and hybrid shish-kebab structures for various polymer-filler systems ${ }^{1-5}$. Here the filler typically provides a nucleating surface that promotes crystallization of the respective polymer matrix. Examples of interfacial crystallization have also been reported in homocomposites, i.e. composites that consist of chemically identical but physically distinct phases separated by an interface. Leong et al. reported an increased interfacial adhesion between polypropylene film and polypropylene matrix when tuning the processing conditions ${ }^{2}$. Qaun et al. observed the presence of interfacial transcrystals in all-polyethylene fiber homocomposites ${ }^{1}$. Ishida et al. also observed transcrystals in ultrahigh-modulus polyethylene fiber-reinforced polyethylene composites ${ }^{6}$. In all cases, the formed interfacial semi-crystalline morphology was decisive in the improvement of interfacial adhesion.

In recent years, the growing need for "green" composites has directed attention to substitutes for traditional plastics as these materials are environmentally compatible without sacrificing performance. In this regard, poly(lactide) (PLA) is seen as a promising candidate for composite materials. PLA-based composites have been reported with different fillers, of which some even induce interfacial transcrystallization at the filler matrix interface ${ }^{7-10}$.

In efforts to produce superior composites, some studies have utilized the stereocomplexation phenomena found in PLAs, which was first reported in the 1980 s by lkada et al. ${ }^{11}$. They observed the presence of a unique crystal phase that was formed by the interaction of enantiomerically opposite poly(L-lactide) (PLLA) and poly(D-lactide) (PDLA). To recall, the blending of PLLA and PDLA results in stereocomplex or racemic crystallite formation, resulting in a melting temperature of nearly $230^{\circ} \mathrm{C}$, approximately $50^{\circ} \mathrm{C}$ higher to the homocrystals of PLLA or PDLA. The difference in melting temperature provides a route to prepare PLA based materials with enhanced performance including mechanical strength, thermal stability and hydrolytic resistance ${ }^{12,13}$.

Habibi et al. were the first to utilize stereocomplex crystals at filler matrix interfaces by grafting PDLA on cellulose nanocrystals (CNC) creating nanohybrid structures ${ }^{14}$. Once blended in a PLLA matrix, it resulted in the co-crystallization of grafted PDLA chains and unbounded PLLA chains in the matrix to produce interfacial stereocomplex crystals. Recently, Ma et. al. utilized a similar concept of PDLA chains grafted on MWCNTs and dispersed in PLLA matrix to produce $-82-$ 
conductive nanocomposites ${ }^{15}$. Examples of interfacial stereocomplexation can also be found for PLA homocomposites. For example, Arias et al. reported that stereocomplex crystals could be produced at the interface of PLLA matrix and PLA particles consisting of chains with sufficiently long $D$-lactide units ${ }^{16}$. These particles were produced by spray droplet atomization and dispersed in the poly(lactide) matrix by melt extrusion. Their results indicated that the interaction between matrix-filler increased due to interfacial stereocomplex crystals that translated to increased stiffness and tensile strength. Additionally, stereocomplex crystals were also used to induce the formation of interfacial transcrystal morphology as demonstrated by Wen et al. and Weng et al. in PLLA-stereocomplex fiber composites ${ }^{17,18}$. Here, high nucleation efficiency of stereocomplex fibers results in the development of continuous transcrystalline PLLA a crystals on their surface.

The concept of interfacial stereocomplexation has also been adapted to some processing techniques. Chen et al. utilized a multi melt injection molding (M3IM) system to produce an interfacial stereocomplex band by inter-penetrating PLLA and PDLA melts ${ }^{19}$. Akagi et al. used layer by layer stepwise deposition of PLLA and PDLA by inkjet printing to produce stereo crystals on a substrate ${ }^{20}$. Recently, we utilized a twin nozzle Fused Deposition Modelling (FDM) system to produce interfacial stereo crystals by alternating deposition of PLLA and PDLA melts ${ }^{21}$. The results showed significant improvement in interfacial stiffening and nucleation of homocrystals in the spatial vicinity of the stereocomplexed interface. Additionally, fundamental insights into the rate and spatial distribution of interfacial stereocomplexation in a single macroscopic melt interface, under isothermal conditions, were derived via rheometry and synchrotron wide-angle X-ray diffraction tomography. Depending on the respective processing strategies summarized above, both the time- and length-scales of interfacial stereo crystallization varied distinctly; from seconds to hours and from nano- to a few hundreds of micrometer length-scales.

In this study, the understanding of interfacial stereocomplexation in heterogeneous PLLA and PDLA melt interfaces is expanded by questioning: what is the effect of $(a)$ the molar mass ratio of the enantiomerically opposite poly(L-lactide) and poly(D-lactide), and (b) temperature on the interfacial diffusion and stereocrystallization kinetics? Homogeneously solid state mixed powders of PLLA and PDLA with a 200 to $250 \mu \mathrm{m}$ size distribution were (pre)compacted and studied under isothermal conditions to monitor interfacial stereocomplexation. While rheometry and differential scanning calorimetry provided insight in the kinetics of interfacial stereocomplexation, polarized optical microscopy and spatially resolved FTIR imaging revealed morphological evolution. Ultimately, the viability of interfacial stereocomplexation for a powder-based additive manufacturing approach like Selective Laser Sintering (SLS) is explored. 


\subsection{MATERIALS AND METHODS}

\subsubsection{Materials}

The polymeric materials used in this study are poly(L-lactide) and poly(D-lactide) with enantiomeric purities above $99 \%$ and kindly provided by Corbion Purac. The molar mass characteristics based on gel permeation chromatography using HFIP as eluent and PMMA $100000 \mathrm{~g} / \mathrm{mol}$ standards are summarized in Table 5.1 .

Table 5.1: Number average molecular weight $\left(M_{n}\right)$, weight average molecular weight $\left(M_{w}\right)$ and Polydispersity (PDI) for different PLLA and PDLA grades used in the study.

\begin{tabular}{|l|l|l|l|}
\hline PLA & $M_{\mathrm{n}}(\mathrm{kg} / \mathrm{mol})$ & $\mathbf{M}_{\mathrm{w}}(\mathrm{kg} / \mathrm{mol})$ & PDI \\
\hline PLLA229 & 107 & 229 & 2.10 \\
\hline PLLA170 & 82 & 170 & 2.07 \\
\hline PLLA140 & 66 & 140 & 2.12 \\
\hline PDLA90 & 48 & 90 & 1.80 \\
\hline
\end{tabular}

\subsubsection{Preparation of blends}

All materials were dried at $70^{\circ} \mathrm{C}$ under vacuum prior to use. The PLLA and PDLA grades were ground into powders cryogenically using a Fritsch Pulviresette 14 . The powder fraction in the range of $200-250 \mu \mathrm{m}$ was sieved out and used for further sample preparation. A mixture of PDLA and PLLA in a weight ratio of $1 / 1$ was prepared by tumble mixing of the powders at room temperature. From this mixture, discs of $12 \mathrm{~mm}$ diameter and $1.5 \mathrm{~mm}$ thickness were compression molded at $160^{\circ} \mathrm{C}$ for $10 \mathrm{~min}$ and a pressure of 10 bars. A temperature of $160^{\circ} \mathrm{C}$ matches the onset temperature of melting, Figure 5.1, and prevents interfacial pre-mixing and stereocrystallization, while sufficiently compacting the powder particles to minimize the presence of voids as supported by Scanning Electron Microscopy, Figure 5.2, particularly once being loaded and molten in the rheometer. Compaction at temperatures above $160^{\circ} \mathrm{C}$ induces interfacial pre-mixing of the PLLA and PDLA chains and consequential formation of stereocomplex crystals or stereocrystals, as seen in Figure 5.3. Table 5.2 summarizes the studied samples, sample codes and corresponding molar mass ratio. 


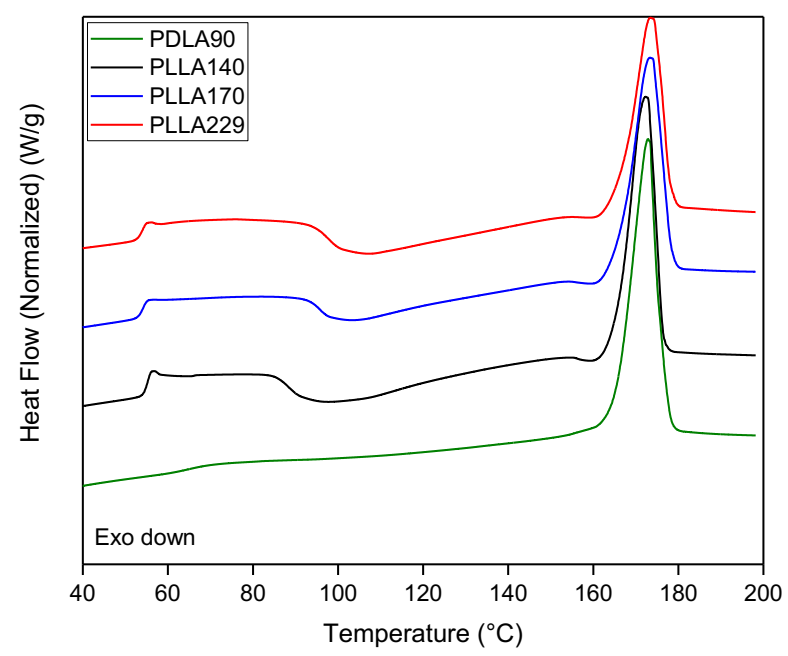

Figure 5. 1: DSC thermograms of the poly(lactide) powders prepared by cryogenic grinding. Samples were heated to $200^{\circ} \mathrm{C}$ at a rate of $10^{\circ} \mathrm{C} /$ min under nitrogen atmosphere.

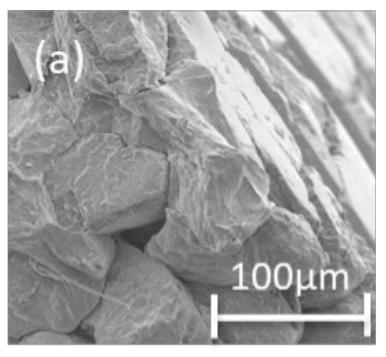

$150^{\circ} \mathrm{C}$

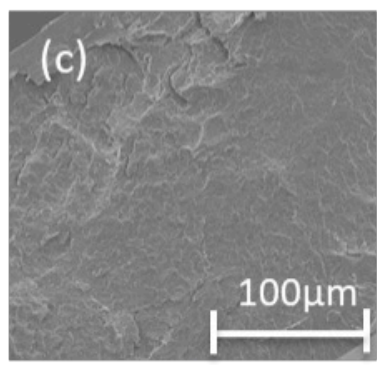

$170^{\circ} \mathrm{C}$

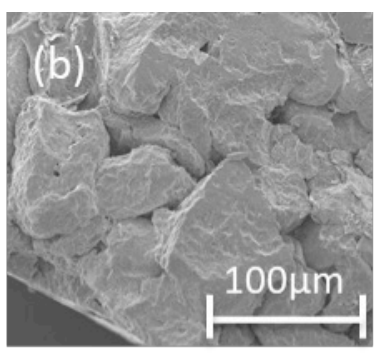

$160^{\circ} \mathrm{C}$

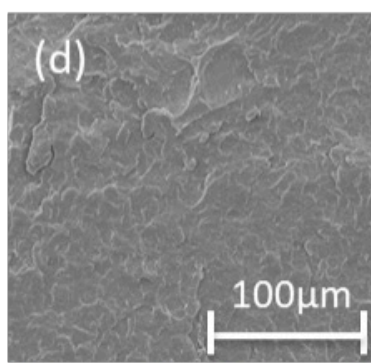

At $t_{0}$ of isothermal rheometry measurement

Figure 5.2: SEM micrographs of sample discs prepared by compression molding of PLLAL140 and PDLA90 powders at (a) $150^{\circ} \mathrm{C}$, (b) $160^{\circ} \mathrm{C}$ and (c) $170^{\circ} \mathrm{C}$, all at 10 bars of pressure. The SEM micrographs reveal that as the compaction temperature increases, the internal void fraction decreases, but simultaneously increases the chance of premixing at the powder interfaces due to melting. Figure (d) shows the crosssection of a sample disc that was precompacted at $160^{\circ} \mathrm{C}$ and 10 bars after being further compacted in the rheometer using a force of $3 \mathrm{~N}$ before the isothermal measurement, demonstrating the absence of voids during the rheological measurements. 


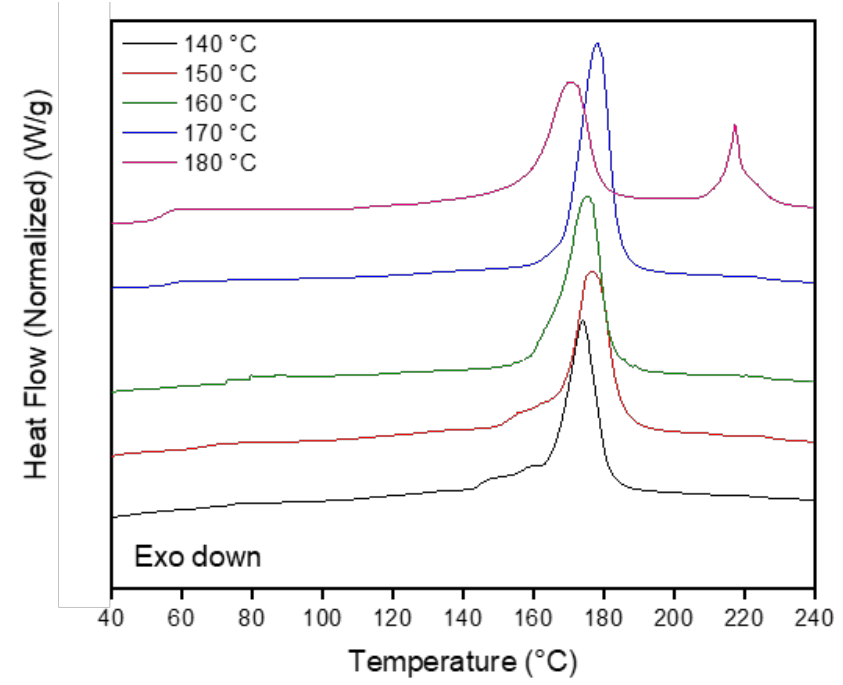

Figure 5.3: DSC thermograms of PLLA140-PDLA90 sample discs prepared by compression molding at different temperatures. The discs were successively heated in DSC to $250^{\circ} \mathrm{C}$ at a heating rate of $10^{\circ} \mathrm{C} / \mathrm{min}$, probing molecular mixing and sterecomplexation during sample preparation. No endothermic melting peak for stereocomplex crystals formation (represented by an endotherm at approximately $220^{\circ} \mathrm{C}$ ) could be detected, up to $170^{\circ} \mathrm{C}$. The disc molded at $180^{\circ} \mathrm{C}$ shows stereocomplexation as clearly witnessed by an endotherm at $220^{\circ} \mathrm{C}$. From these findings and the resultant ease in compaction at $160^{\circ} \mathrm{C}$, this temperature was chosen for further studies.

Table 5.2: Sample coding based on blend compositions and correlated molar mass ratio utilized in the study.

\begin{tabular}{|c|c|c|c|}
\hline Sample code & PLLA (wt \%) & PDLA (wt\%) & molar mass ratio \\
\hline L229-D90 & 50 & 50 & 2.6 \\
\hline L170-D90 & 50 & 50 & 1.9 \\
\hline L140-D90 & 50 & 50 & 1.6 \\
\hline
\end{tabular}

\subsubsection{Isothermal crystallization}

Interfacial crystallization in the prepared blends was investigated via Differential Scanning Calorimetry (DSC) and rheometry. DSC was performed using a TA instruments Q 2000 DSC. With $\pm 5 \%$ accuracy, $5 \mathrm{mg}$ of sample was sealed in aluminum pans and heated at a rate of $10^{\circ} \mathrm{C} / \mathrm{min}$ from room temperature to the targeted stereocrystallization temperature above the melting point of the homopolymers and held isothermally. After the completion of the isothermal event, the sample was heated at $30^{\circ} \mathrm{C} / \mathrm{min}$ to $250^{\circ} \mathrm{C}$ to melt the stereocrystals that had formed. From this step, the melting enthalpy of the stereocrystals $\left(\Delta \mathrm{H}_{\mathrm{m}}\right)$ was calculated, directly indicative of the amount of stereocrystals produced during the isothermal step.

Dynamic melt rheological measurements were performed on the compression molded discs using an Anton Paar MCR 302 rheometer with parallel plate geometry. A plate diameter of $12 \mathrm{~mm}$ was used. The samples were heated to $170^{\circ} \mathrm{C}$ at a rate of $10^{\circ} \mathrm{C} / \mathrm{min}$. A normal force of $3 \mathrm{~N}$ was applied during this step to remove potential voids as supported by SEM, (Figure 5.2(d)). After this step, -86 - 
the sample was heated to the desired crystallization temperature at $30^{\circ} \mathrm{C} / \mathrm{min}$ without the application of a normal force. On reaching the targeted stereocrystallization temperature, the sample was kept isothermally for 60 minutes. After this step, the sample was cooled to room temperature, at a cooling rate of $10^{\circ} \mathrm{C} / \mathrm{min}$. The discs were retrieved and used for further analysis.

\subsubsection{Analysis of the crystalline morphology}

To study the crystalline morphology generated during the rheometry experiments, thin slices of $2.5 \mu \mathrm{m}$ were cut from the discs using a Leica EM UC7 ultra-microtome. Polarized Optical Micrographs (POM) of the slices were taken between cross-polarizers on an Olympus BX53 microscope mounted with an Olympus DP26 camera. Fourier Transform InfraRed (FTIR) imaging of the identical sample slices were recorded in transmission mode on a Perkin Elmer Spotlight $400 \mathrm{FT}(\mathrm{N})$ IR microscope. Absorbance imaging was performed on a $350 \mathrm{~mm} \times 350 \mathrm{~mm}$ area with a pixel size of $6.25 \mu \mathrm{m}$, accumulating 16 scans in a spectral range of $1000-800 \mathrm{~cm}^{-1}$ and $2 \mathrm{~cm}^{-1}$ spectral resolution.

\subsubsection{Selective Laser Sintering}

Interfacial stereocomplexation under non-isothermal Selective Laser Sintering (SLS) conditions was evaluated by preparing a single layer using homogeneously solid state blended powders of an average size of $100 \mu \mathrm{m}$. Printing was performed on a Sharebot Snowwhite SLS printer employing a bed temperature of $160^{\circ} \mathrm{C}$ to suppress homocrystallization after laser-induced melting, a laser spot of $0.1 \mathrm{~mm}, 4000 \mathrm{~mm} / \mathrm{s}$ laser speed and a laser power of $5.67 \mathrm{~W}$. The geometry of the single layer was based on a tensile test geometry of ISO 6892 .

\subsection{RESULTS AND DISCUSSION}

\subsubsection{Interfacial stereocomplexation as a function of molar mass ratio}

The stereocomplex formation at the interface of molten PLLA and PDLA was investigated by oscillatory shear rheometry at $200^{\circ} \mathrm{C}$. In Figure 5.4(a) the evolution of the storage modulus (G'), which represents the mechanical stiffening due to stereocrystallization ${ }^{19}$, is followed at $200^{\circ} \mathrm{C}$ as a function of time for different molar mass ratios. To support that the increase in $G$ ' is associated with the formation of stereocrystals, additional samples were produced in which the PDLA fraction (D90) was replaced by a PLLA of the same molar mass (L90). These blends are referred to as PLLA-PLLA blends and are represented by dashed lines in Figure 5.4(a). As anticipated no increase in G' after one hour is observed for the PLLA-PLLA reference samples.

From Figure 5.4(a) it is evident that at the beginning of the measurement stereocrystals are already present in the melt as the G' for all samples with stereocrystals (PLLA-PDLA blends) is 
higher than the corresponding blends without stereocrystals (PLLA-PLLA blends). In fact, G' of the L140-D90 combination is higher than for L170 -D90 and L229-D90 combination at $\mathrm{t}_{0}$, which indicates that the extent of interfacial stereocomplexation $a t_{0}$ is the highest for the lowest molar mass ratio. The build-up in $G^{\prime}$ increases faster at the beginning of the experiment than in the later stages. A two-stage process, driven by chain dynamics, was also reported in the diffusion and consequential mixing of polymer chains in poly(styrene)-poly(styrene) "weld" interfaces by Bousmina et al. ${ }^{22}$. Due to the identical nature of the polymers studied in the polystyrene blends with the absence of interfacial stereocrystallization, the increase in G' as a function of time was attributed to chain dynamics. To recall, different time regimes in the modulus build-up was assigned as follows. In the first stage, $\mathrm{G}^{\prime}$ increases proportionally to $t^{1 / 2}$, correlating to Rouse motion, whereas in the second stage reptation causes $G$ ' to increase proportionally to $t^{1 / 4}$. However, since Rouse motion occurs in the order of seconds and at angstrom to nanometer length-scales, it cannot be accounted for the progressive increase in G' over time-scales of minutes as observed in Figure 5.4(a) for the PLLA-PDLA blends. In Figure 5.4(a), where stereocomplexation is a dominant factor the changes in the storage modulus are likely to be dominated by crystals formation in the melt. Also, polymer crystallization proceeds via a two-stage process as extensively reported by Zachmann and Stuart, introducing a distinction between primary (main) and secondary (subsequent) crystallization ${ }^{23}$. In this theory, the transition from high to low crystallization rate is assigned to an initial fast crystal growth that is concluded at the moment of impingement of crystallites/spherulites. Successive crystallization (secondary) proceeds via spacefilling, via thickening of the crystals, growth of new lamellae (stacks), and crystal refinement facilitated by the remaining amorphous regions within the spherulites. To analyze the time dependent behavior for interfacial stereocrystallization in more detail, fits were added to both stages of the measurement as illustrated in Figure 5.4(b). These fits obey the exponential relation $G^{\prime}=G_{0}^{\prime} e^{-B t}$; where $G^{\prime}{ }_{0}$ is the storage modulus at $t_{0}$ and $B$ is the rate coefficient that describes the rate of change of $G$ '. For these fits, the rate constant at the beginning of the experiment (B1) and in the latter part of the experiment (B2) were calculated for all blends and are shown in Figure 5.4(c). 

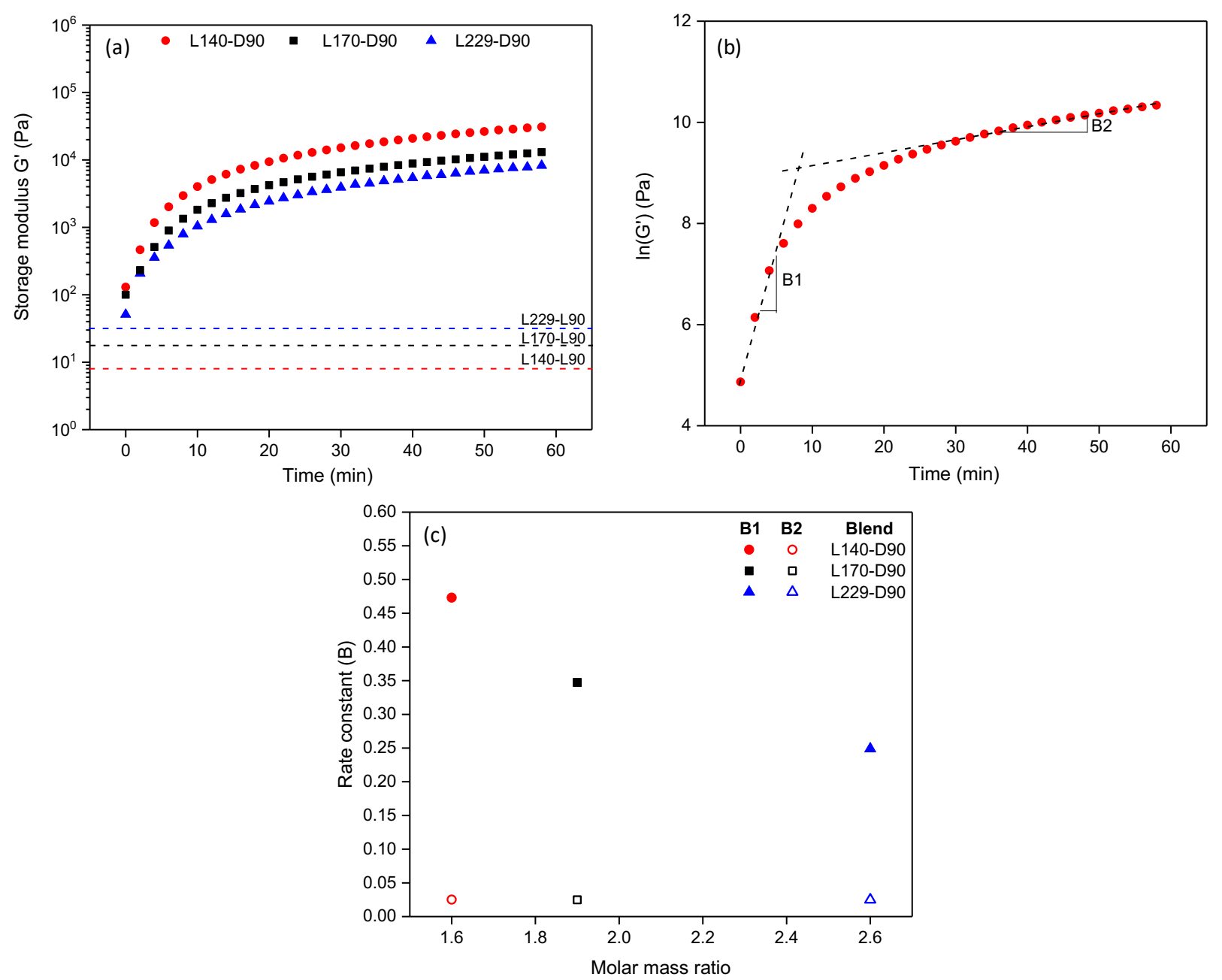

Figure 5.4: Increase in storage modulus as a function of time under the isothermal condition in the linear viscoelastic regime. (a) Storage modulus $\left(G^{\prime}\right)$ at $1 \mathrm{rad} / \mathrm{s}$ of precompacted molten disk made with opposite (symbols) and opposite (dashed) enantiomeric compositions as a function of time to probe the kinetics of interfacial stereo complexation upon varying molar mass ratio at $200{ }^{\circ} \mathrm{C}$; (b) exponential fits for the beginning and the latter part of the isothermal measurement illustrating rate constant B1 and B2 for L140-D90 blend ;(c) Rate constant B1 and B2 plotted as a function of molar mass ratio. The letters and numbers of sample encoding represent the enantiomer and molar mass of the mixed powders respectively.

For interfacial stereocomplexation to occur molecular mixing and thus diffusion of one stereospecific PLA into the other and vice versa is necessary prior to crystallization. The diffusivity (D) of polymers in the melt is inversely related to the molar mass (M) by $D \propto M^{-2}$ as described by the reptation theory ${ }^{24}$. Since in this experiment the PDLA fraction remains the same for all blends, the effective molar mass of the blend increases with the molar mass ratio. Hence, it is expected that the sample with the lowest molar mass ratio shows the highest rate of stereocomplexation. This hypothesis can be proven by following B1 as a function of molar mass ratio (Figure 5.4(c)). Indeed, B1 decreases with an increase in the molar mass ratio. The effective stiffening of the interface by stereocomplexation, as depicted by the absolute G' 
against the $G^{\prime}$ of the PLLA-PLLA reference blends ( $\left.\Delta G^{\prime}\right)$, is inversely related to the molar mass ratio. Interestingly, the rate of stereocomplexation at the later stages of the experiment appears to be independent of the molar mass ratio, as seen by the rate constants B2. It is likely that upon stereocomplexation a barrier to interfacial diffusion is progressively formed as time progresses, making the rate of stereocomplexation independent of molar mass ratio. Moreover, it is to be noted that the onset times for reduction in the rates of stereocomplexation increase with increasing molar mass ratio, which fits the hypothesis that the formation of a diffusion barrier is related to the progressive formation of stereocrystals. Since $\Delta G^{\prime}$ is proportional to the fraction of stereocrystals, the extent of diffusion seems to be dependent on molar mass ratio and is set prior to the formation of the diffusion barrier. This trend is further investigated by morphological studies reported below.

After cooling within the rheometer, the rheologically investigated samples were microtomed into $2.5 \mu \mathrm{m}$ thick slices and were studied by Polarized Optical Microscopy (POM). The optical micrographs are given in Figure 5.5. At first glance, the micrographs reveal that all samples typically contain two types of domains, one domain marked by birefringent larger crystals and the other constituting of less birefringent smaller crystals. For poly(lactides) in the studied molar mass regime, the homocrystallization rate is largely molar mass dependent, inducing increased crystallite sizes with increasing molar mass ${ }^{25}$. Consequently, large crystallites represent the PLLA phase while small crystallites mark the PDLA phase. Additionally, the dark areas separating the two domains are likely to be the interfacial area separating the enantiomerically opposite phases. Are stereocrystals detectable, and if so, what is their spatial distribution? To answer these questions, FTIR absorbance images are constructed via FTIR imaging of the optically identified sample area. It is known that the PLA chain conformation in stereocrystals results in a distinctive vibrational band at $908 \mathrm{~cm}^{-1} 26,27$. The band at $921 \mathrm{~cm}^{-1}$ is assigned to the coupling of $\mathrm{C}-\mathrm{C}$ backbone stretching with the $\mathrm{CH}_{3}$ rocking mode and is representative of the $10_{3}$ helical chain conformation of a homocrystals that occur in both PLLA and PDLA ${ }^{26,28}$. Based on these distinctive vibrational modes, the regions containing the characteristic $908 \mathrm{~cm}^{-1}$ band of stereocrystals have been highlighted in the FTIR absorbance images. The highlighted regions perfectly match the dark interface between the two bright spherulitic domains observed in POM, spatially localizing the stereocrystals. The dark regions in the absorbance images are marked by the characteristic peak of the homocrystals only, enabling determination of the length-scales or bandwidth of interfacial stereocomplexation. 


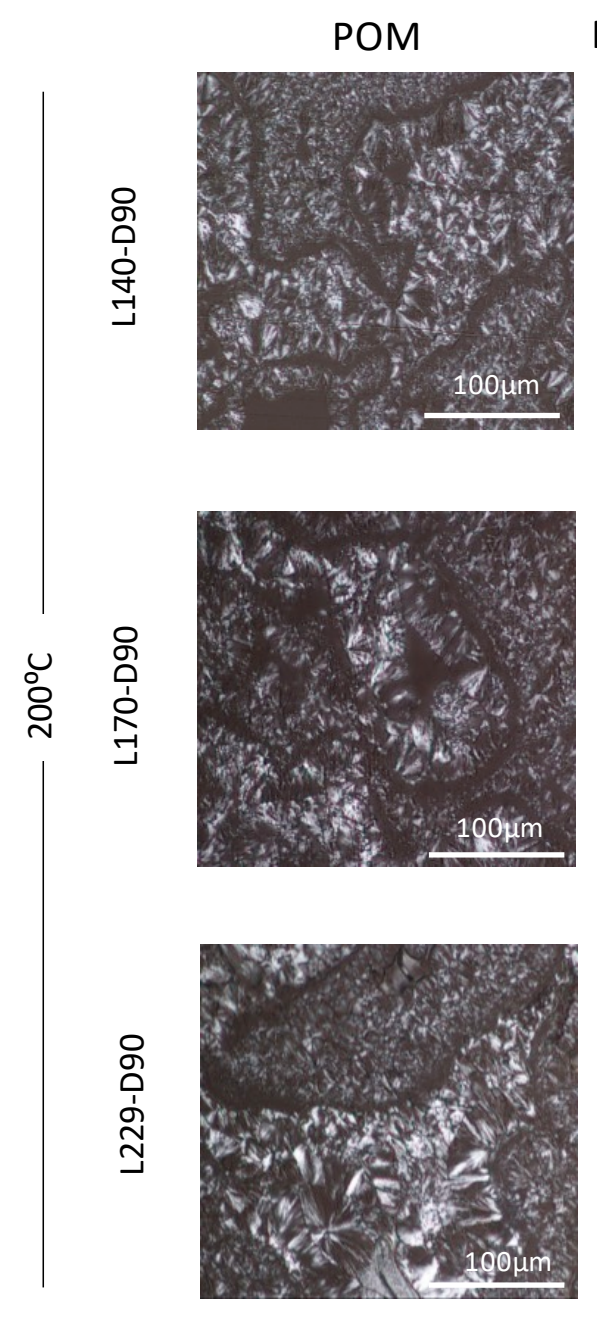

(a)
FTIR absorbance image
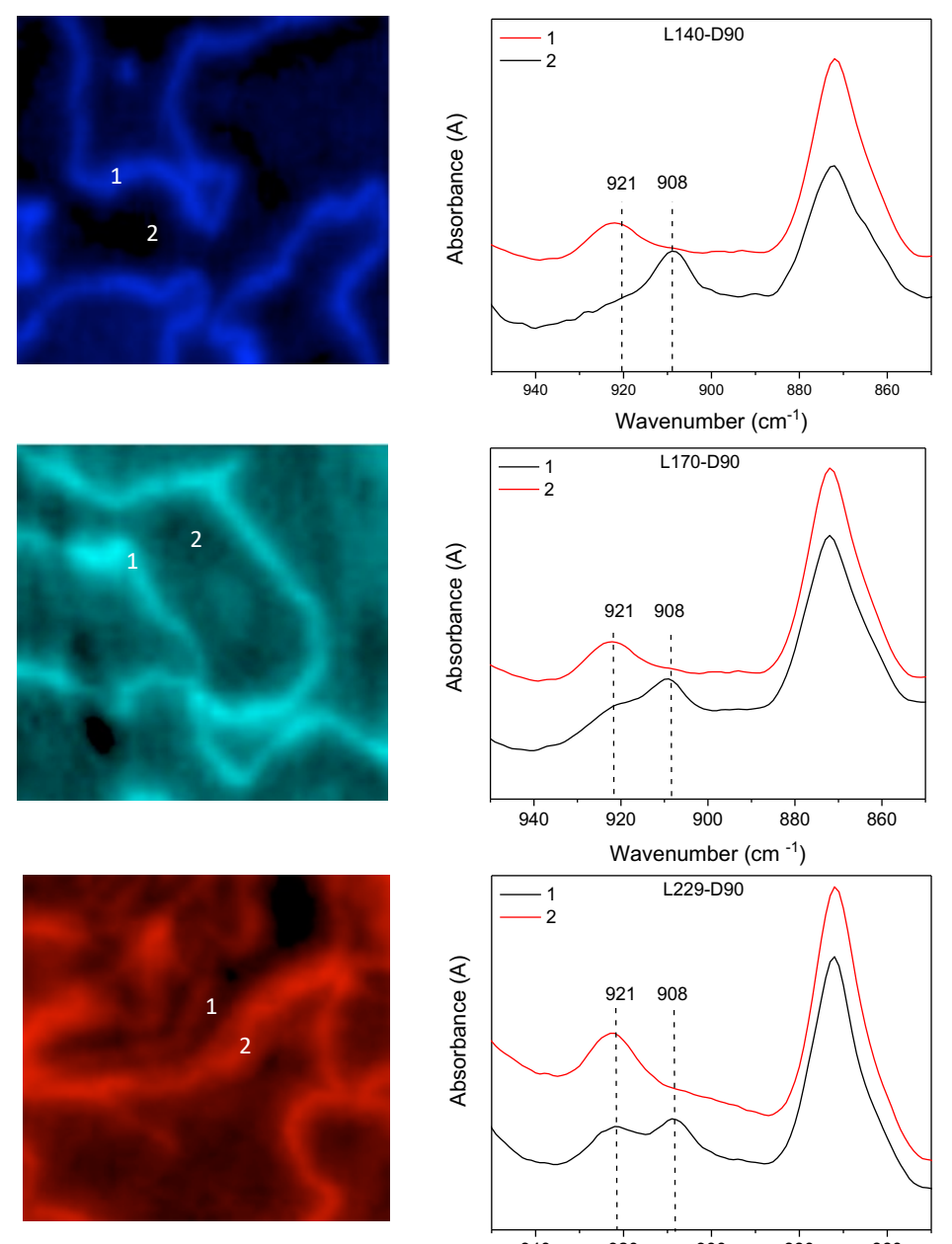

(b)

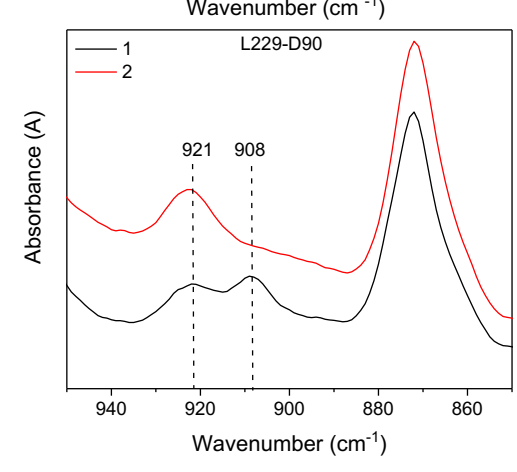

(c)

Figure 5.5: Interfacial crystalline morphology obtained after cooling from the isothermal rheometry measurement at $200^{\circ} \mathrm{C}$ observed in (a) polarized optical microscopy (POM) and (b) corresponding FTIR absorbance images highlighting the structural composition of the interfaces produced. Note that the scale bars for POM and FTIR imaging are identical. The absorbance spectra of region 1 (at the interface) and region 2 (aside the interface) are plotted in (c). The presence of stereocrystals is confirmed by the characteristic $908 \mathrm{~cm}-1$ band in region 1 (black spectra) and not in region 2 (red spectra).

In Figure 5.6(a) the ratio of stereo- to homocrystals based on peak height, $A_{908} / A_{921}$, is plotted across the bandwidth of the interfacial region for the different molar mass ratios. To characterize the directionality of diffusion caused by the higher diffusivity of the lower molar mass PDLA, the $A_{908} / A_{921}$ ratio is determined by crossing the interface from the pure PLLA (negative bandwidth values) to PDLA zones (positive bandwidth values). Consequently, an asymmetry in the spatial distribution of stereocrystals may exist, but it is cancelled out by fitting a Gaussian curve to the points as a guide to the eye. The bandwidth is a direct indicator of the length-scales of diffusion that decreases from $30.0 \pm 1.7$ to $18.6 \pm 1.7$ to $12.3 \pm 1.8 \mu \mathrm{m}$ 
with the increase in molar mass ratio; though the thermodynamic driving force for stereocrystallization $(\Delta G)$ is likely to be independent of the molar mass. Thus, the cause of the bandwidth dependence on the molar mass ratio may be attributed to the kinetics involved in chain diffusion, which is likely to arise from relative variations in viscosity, determining the bandwidth and relative population of stereo- and homocrystals within. The height of the Gaussian curve attributes to the relative amount of stereocrystals at the interface, as indicated by the absorbance ratio $908 / 921$ in Figure 5.6(a). In Figure 5.6(b) the melting enthalpy of the stereocrystals $\left(\Delta \mathrm{H}_{\mathrm{m}}\right)$, representing the total amount of stereocrystals independent of the spatial distribution, is plotted as a function of molar mass ratio. Normalization of the melting enthalpy to the bandwidth results in an average amount of stereocrystals per unit width, being 4.7, 5.1 and 5.1 with increasing relative molar mass. This implies that the total amount of stereocrystals formed depends only slightly on the length-scale of molecular diffusion and mixing, and thus indeed relative molar mass induced viscosity variations and ultimately diffusivity.
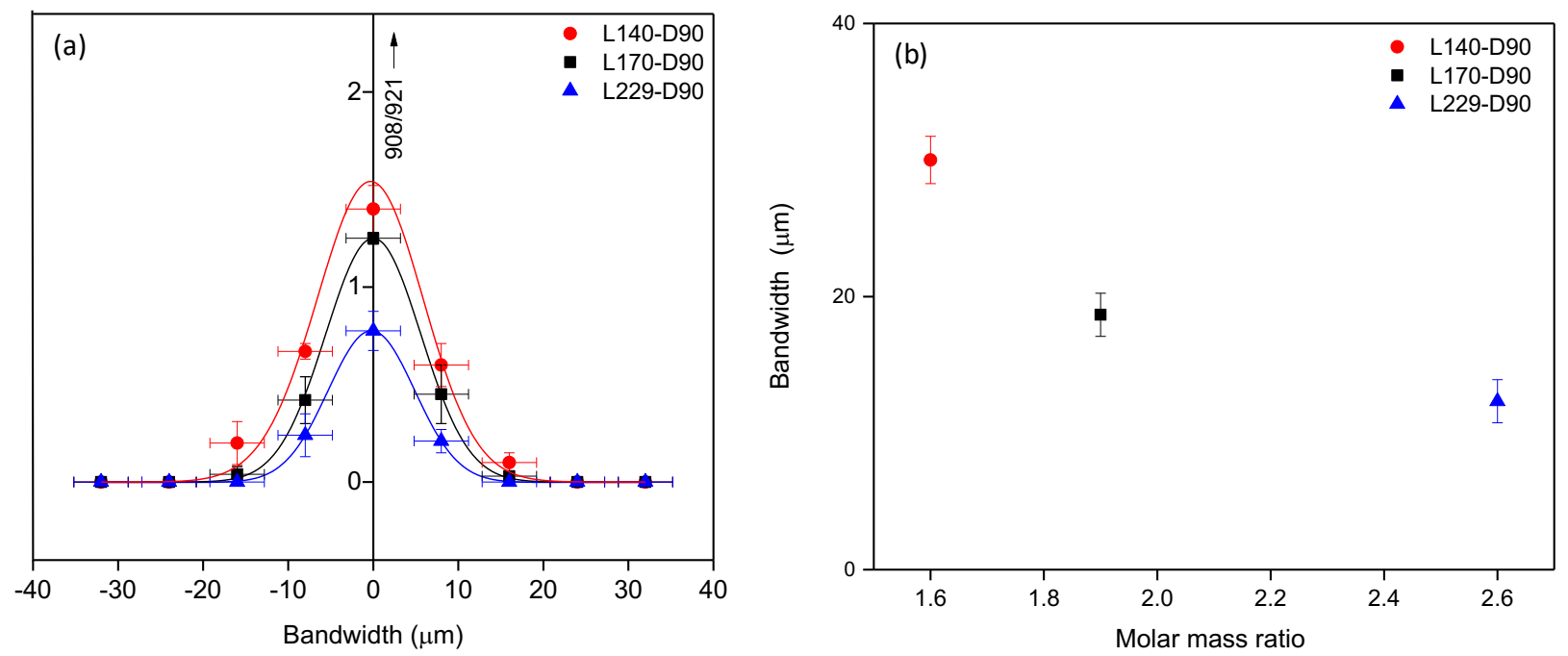

Figure 5.6: (a) The averaged absorbance ratio of the stereo- and homocrystal bands $\left(908 \mathrm{~cm}^{-1} / 921 \mathrm{~cm}\right.$ $1)$, directionally measured from the PLLA $(w<0)$ to the PDLA $(w>0)$ across the average bandwidth $w$ for varied molar mass ratios; (b) The enthalpy of melting of interfacial stereocrystals formed during isothermal crystallization at $200^{\circ} \mathrm{C}$ plotted as a function of molar mass ratio.

The $A_{908} / A_{921}$ distribution curve, Figure 5.6(a), shows that for the L140-D90 combination the ratio of stereo- to homocrystals in the center of the bandwidth is greater than one, indicating that the majority of the PLLA and PDLA chains are involved in stereocomplex formation. Though for the L170-D90 blend the $A_{908} / A_{921}$ ratio decreases, still more stereo- than homocrystals are present. However, for the L229-D90 blend, which has the highest difference in molar mass between the PLLA and PDLA fraction, more homocrystals than stereocrystals are observed. 
Previous studies on melt blended PLLA/PDLA racemic compositions have shown that molar mass has a profound impact on the stereocomplexation process ${ }^{29,30}$. As the molar mass ratio of PLLA and PDLA increases, the degree of stereocomplexation decreases significantly, and homocrystallization becomes predominant. But, if stereocrystallization is promoted by low molar mass and the thermodynamic driving force for crystallization is identical for all molar mass ratios, why does the overall mechanical stiffening $\Delta G^{\prime}$ (G'PDLA-PLLA - G'PLLA-PLLA), Figure 5.4(a), decreases with an increase in the molar mass ratio? How chains in the sample with the lowest molar mass and highest stereocrystallization rate diffuse across the largest length-scale? A direct correlation to interfacial stereocrystallization may not be valid as it depends on the degree of mixing of the enantiomerically opposite chains at the interface. In fact, since both stereo-and homocrystals are present in the center of the bandwidth (Figure 5.6(a)) and $\Delta G^{\prime}$ is smallest for the highest molar mass ratio (L229-D90), crystallites acting as a physical barrier for chain diffusion cannot be the primary cause for the change in the rate of modulus build-up. To understand the cause of the change in the rate of the modulus build-up, the role of complex viscosity on the modulus build-up is investigated.

Like the time-resolved variation in G' upon interfacial stereocrystallization, the complex viscosity at $200^{\circ} \mathrm{C}$ is plotted as a function of time for the three molar mass ratios, Figure 5.7. Figure 5.7, similar to Figure 5.4(a), shows two distinct slopes. The onset time for the change in slope (deflection) increases with increasing molar mass ratio. However, the complex viscosity seems to be in the same order of magnitude at the deflection point, suggesting that at a critical complex viscosity the network formation arising with the stereocomplexation (similar to gelation) occurs. Due to the initially lower complex viscosity for the lowest molar mass ratio that originates in fewer entanglements per chain in the PLLA phase, more crystals are needed for gelation. Realizing that $G^{\prime}=\frac{\rho R T}{\left\langle M_{c}\right\rangle}$, where $M_{c}$ is the molar mass between physical crosslinks (being here stereocrystals mainly), the relatively large amount of crystals per chain being formed in the low molar mass ratio sample increases $\Delta G^{\prime}$ to a larger extent in comparison to the higher molar mass ratios. Stereocrystals have been reported to act as rheology modifiers enhancing the melt strength of PLA, even if present in small concentrations. For example, Saeidlou et al. showed that the addition of only $3 \mathrm{wt}-\%$ PDLA in a PLLA rich melt induces a remarkable increase in viscosity and elasticity due to the formation of stereocrystals ${ }^{31}$. Wei et al. demonstrated the stereocrystals formation in the melt of PLLA-PDLA having a very high molar mass ratio that results in the network formation, which is reported as a transition from the liquid-like to solid-like viscoelastic behaviour ${ }^{32}$. Thus, with the stereocomplexation, resulting in a network formation at the interface, further chain diffusion independent of the molar mass will be impeded. This is apparent from the B2 independence of the molar mass ratio, Figure 5.4(c). 


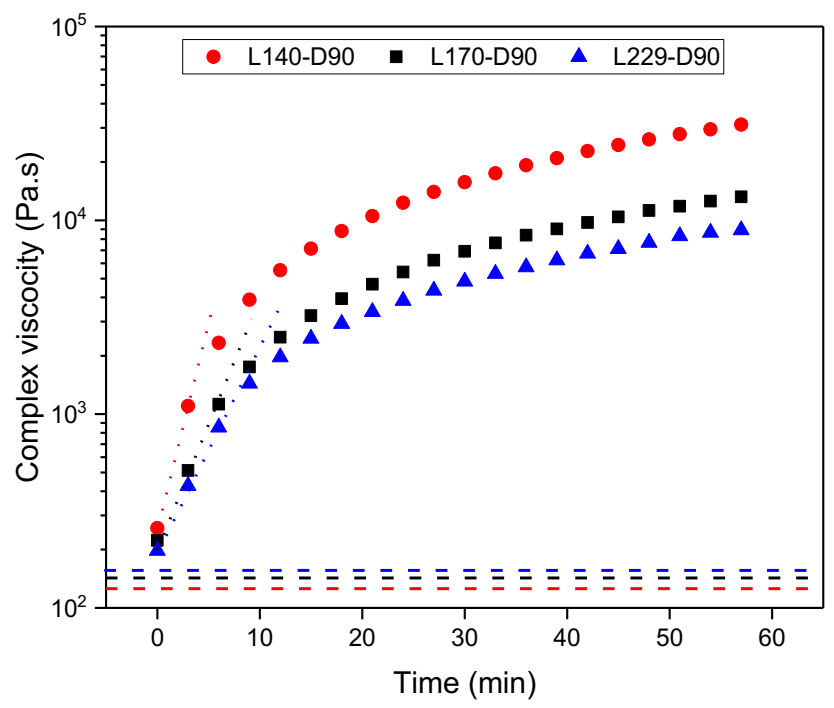

Figure 5.7: Complex viscosity at $1 \mathrm{rad} / \mathrm{s}$ of precompacted molten disk made with opposite (dots) and identical (dashed) enantiomeric compositions as a function of time to probe the kinetics of interfacial stereo complexation upon varying molar mass ratio at $200{ }^{\circ} \mathrm{C}$. The letters and numbers of the sample codes represent the enantiomer and molar mass of the combined powders respectively.

To follow the development of interfacial stereocrystallization from a thermodynamic and structural perspective, the buildup in G' for the L140-D90 combination, Figure 5.4(a) is complemented by DSC and FTIR imaging at different moments in time. In Figure 5.8(a) the enthalpy of melting $\left(\Delta \mathrm{H}_{\mathrm{m}}\right)$ for stereocomplex crystals created by keeping the L140-D90 sample at $200^{\circ} \mathrm{C}$ (where the homocrystals formation could be avoided) for various isothermal crystallization times is plotted. The resulting curve is identical to the trend observed in rheometry strengthening the assignment of the trend in storage modulus and complex viscosity to interfacial stereocomplexation. Up to 10 minutes, $\Delta H_{m}$ increases rapidly to $6 \mathrm{~J} / \mathrm{g}$ after which the rate of stereocrystallization lowers. Also, the growth of the stereocomplexation band by FTIR imaging features two rate constants, Figure 5.8(b), and demonstrates that the length-scale of stereocomplexation, i.e. the bandwidth, is primarily defined in the initial stages by diffusion as the primary mechanism of molecular mixing across the interfaces. The FTIR images used for the calculation of bandwidth are separately provided in Figure D1 of Appendix D. 

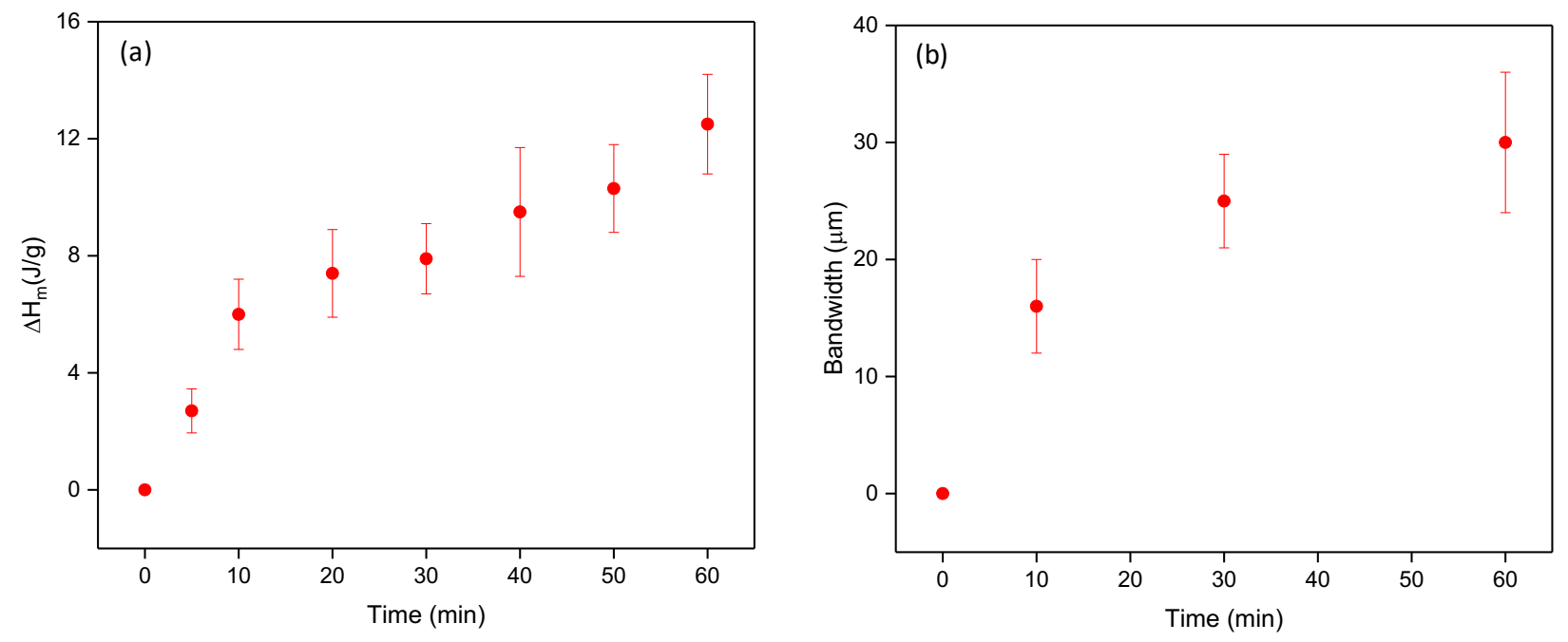

Figure 5.8: (a) Enthalpy of melting of stereocrystals created by isothermal crystallization at $200^{\circ} \mathrm{C}\left(\Delta \mathrm{H}_{\mathrm{m}}\right)$ as a function of crystallization time in DSC. (b) The bandwidth of stereocrystals as determined by FTIR imaging at $0,10,30$, and 60 minutes.

\subsubsection{Interfacial stereocomplexation as a function of crystallization temperature}

So far, the effect of molar mass ratio or relative molar mass and thus relative viscosity on the time- and length-scales of interfacial stereocrystallization at constant temperature has been discussed. In this section, the influence of temperature on the time- and length-scales of isothermal interfacial stereocrystallization is studied for one specific molar mass ratio, L140D90. Similar to crystallization, in general, the crystallization rate is anticipated to be strongly dependent on the supercooling, where the equilibrium melting temperature of PLA stereocrystals is reported to be as high as $279^{\circ} \mathrm{C}^{33-35}$. However, experimentally the melting temperature of stereocomplex is typically in the range of 230 to $240^{\circ} \mathrm{C}$.

Stereocrystallization in melt extruded and compacted powder (solid-state) L140-D90 samples (1:1 wt. ratio) is studied at a defined supercooling in the temperature range of 190 to $220^{\circ} \mathrm{C}$ by DSC. The melt extruded blend of L140-D90 is prepared on a DSM Explore twin-screw co-rotating micro-extruder operating at $240^{\circ} \mathrm{C}, 50 \mathrm{rpm}$ and 1 minute of mixing time. In this blend, the respective polymers are homogeneously mixed in a 1:1 wt. ratio, providing a comparative example for investigating stereocrystallization. The DSC protocol for both blends, depicted in Figure 5.9(a), is defined by (1) heating of the sample to $250^{\circ} \mathrm{C}$ at $10^{\circ} \mathrm{C} / \mathrm{min}$ erasing the thermal history and ensuring the melting of all crystals, (2) isothermal at $250^{\circ} \mathrm{C}$ for 1 minute, (3) cooling to the desired crystallization temperature (Tc) at $30^{\circ} \mathrm{C} / \mathrm{min}$, (4) an isothermal period for 60 minutes, and (5) heating to $250^{\circ} \mathrm{C}$ with $30^{\circ} \mathrm{C} / \mathrm{min}$ to melt the stereocrystals formed, (6) isothermal at $250^{\circ} \mathrm{C}$ for 1 minute and (7) cooling down to $30^{\circ} \mathrm{C}$ with $10^{\circ} \mathrm{C} / \mathrm{min}$. The $\mathrm{DSC}$ results are presented in Figure 5.9(b), where the enthalpy of melting $\left(\Delta H_{m}\right)$ for stereocrystals (step 5) 
is plotted as a function of crystallization temperature (Tc). Here, $\Delta \mathrm{H}_{\mathrm{m}}$ is an indicator of the crystallinity attained after one hour.

It is commonly known that the nucleation rate $(\mathrm{J})$ in polymer crystallization increases exponentially with increasing degree of supercooling, following the equation ${ }^{36}$ :

$$
J=C k\left(r_{c}\right)\left(-\frac{\Delta G^{\prime \prime}\left(r_{c}\right)}{k T}\right)^{1 / 2} e^{-F^{*} / k T}
$$

in which: $\quad C$ is a prefactor reflecting the geometry of the nucleus, $\mathrm{k}\left(\mathrm{r}_{\mathrm{c}}\right)$ is the frequency of arriving repeat units at the critical nucleus, $\Delta \mathrm{G}^{\prime \prime}\left(\mathrm{r}_{\mathrm{c}}\right)$ is the curvature of $\Delta \mathrm{G}$ at $\mathrm{r}_{\mathrm{c}}$, and

$\mathrm{F}^{*}$ is the nucleation barrier to the formation of a critical nucleus with $\mathrm{F}^{*} \sim(\Delta \mathrm{T})^{-2}$, where $\Delta \mathrm{T}$ is the supercooling.

The crystal growth rate, and so the crystallization rate, of semi-crystalline polymers increases initially at low supercooling but decreases while approaching the glass transition temperature upon cooling further. For the melt extruded blend, Figure 5.9(b), no stereocrystallization is observed on annealing at $220^{\circ} \mathrm{C}$. While during annealing at $210^{\circ} \mathrm{C}$ the enthalpy of melting is found to be $10 \mathrm{~J} / \mathrm{g}$, a further reduction in annealing temperature to 200 and $190^{\circ} \mathrm{C}$ increases the melting enthalpy significantly to 97 and $83 \mathrm{~J} / \mathrm{g}$, respectively. The decrease in melting enthalpy on lowering the annealing temperature to $190^{\circ} \mathrm{C}$ is attributed to the increase in the kinetic barrier for stereocomplexation, despite the higher supercooling. For the solid state powder compacted sample, the absolute values of the enthalpy are significantly lower as stereocrystallization only occurs at the interface. At 220 and $210^{\circ} \mathrm{C}$, the enthalpy of melting is 5 and $15 \mathrm{~J} / \mathrm{g}$ respectively, indicating the presence of a small amount of stereocrystals. At these low supercoolings, the enthalpic contribution for melting is very similar to the melt blended samples. However, a remarkable distinction in the melting enthalpy is observed in the samples (melt blended and solid state blended powders), at the higher supercoolings, Figure 5.9(b). The distinction in the enthalpy contribution is associated with the requirement of chain diffusion beyond the interface in the compacted powders. These observations further strengthen that network formation arises with stereocomplexation at the interface. The effect of stereocrystallization on interfacial network formation is investigated by rheometry and discussed in the following paragraph. 

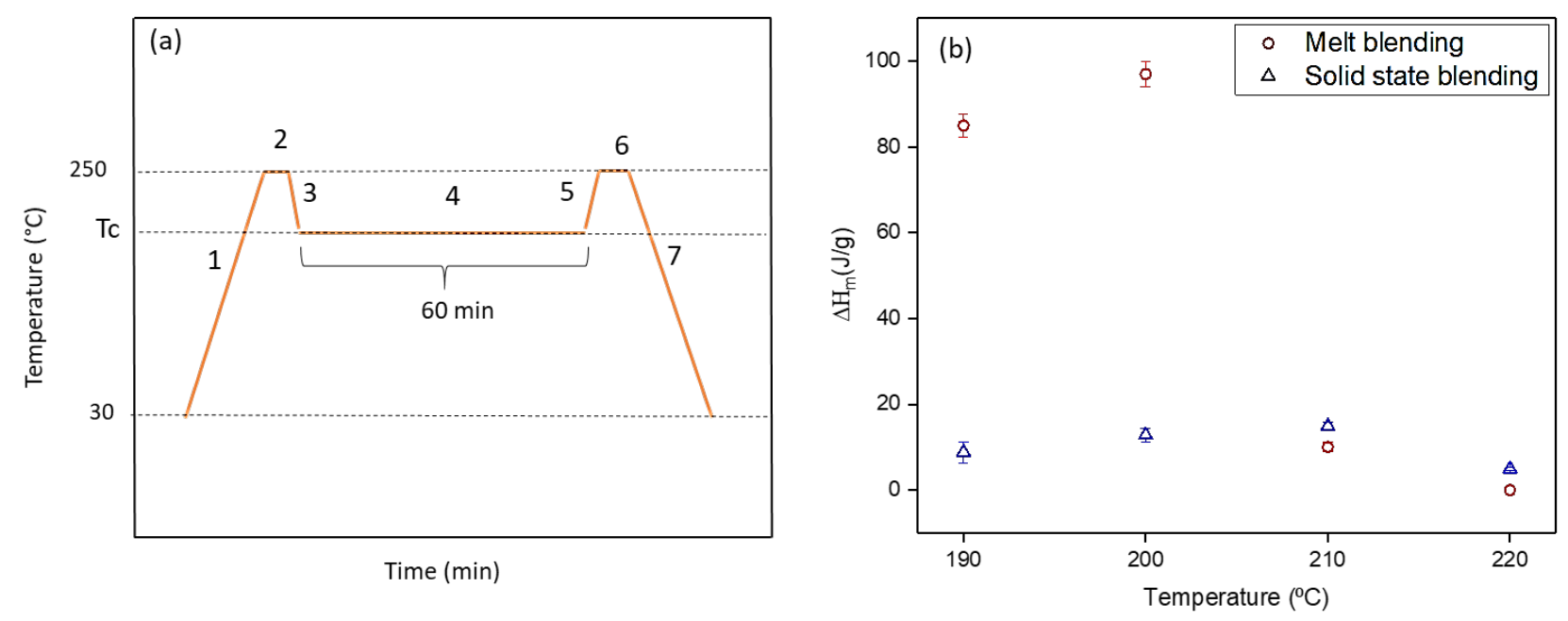

Figure 5.9: (a) Illustration of DSC protocol followed to measure isothermal crystallization for 60 min at different crystallization temperatures (Tc) showing all steps followed (1-7. ); (b) Enthalpy of melting after a 60 minutes isothermal crystallization as a function of temperature for melt blending (extrusion) and (b) solid state blended of PLLA140 and PDLA90 (1:1 wt. ratio).

The rheological response of the melt with the progress of the isothermal stereocrystallization was followed as a function of time, in the linear viscoelastic regime, in a temperature range from 160 to $220^{\circ} \mathrm{C}$. The minimum temperature of $160^{\circ} \mathrm{C}$ was chosen above the onset crystallization temperatures of the homocrystals of PLLA140 and PDLA90, as recorded on cooling from the melt at $10^{\circ} \mathrm{C} / \mathrm{min}$. The recorded onset crystallization temperatures for PLLA140 and PDLA90 are 117 and $112^{\circ} \mathrm{C}$, respectively (Figure D2 of Appendix D). To prevent degradation the maximum temperature for rheological studies, under isothermal conditions was set to $220^{\circ} \mathrm{C}$. The samples that were investigated at or below $190^{\circ} \mathrm{C}$ were heated to $200^{\circ} \mathrm{C}$ prior to the start of the isothermal experiment with the aim to erase the thermal history of the compacted powders of PLLA140-PDLA90. After the isothermal crystallization for 60 minutes, the samples in the rheometer were cooled to room temperature and were investigated further for thermal and structural analysis using DSC, POM and FTIR imaging. 

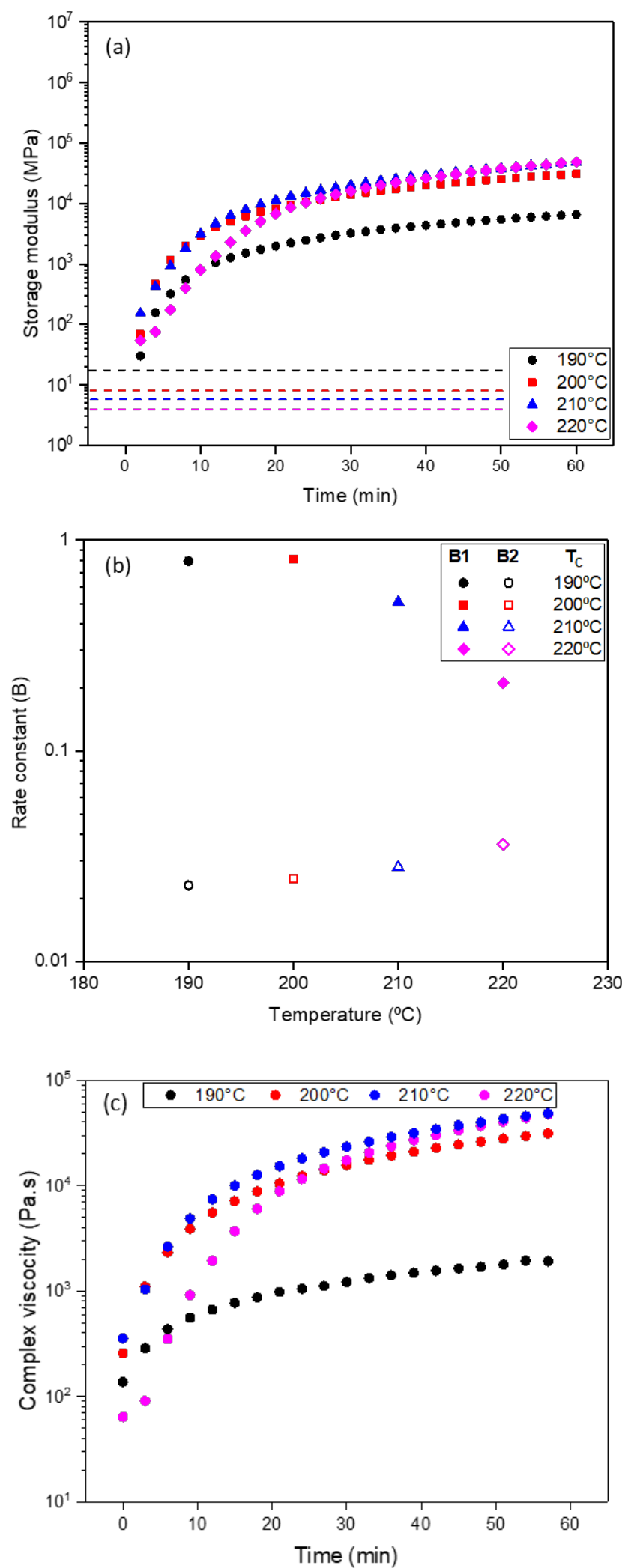

Figure 5.10: a) Storage modulus ( $\left.G^{\prime}\right)$ at $1 \mathrm{rad} / \mathrm{s}$ of precompacted molten disk made with opposite (dots) and identical (dashed) enantiomeric compositions as a function of time to probe the kinetics of interfacial stereocomplexation upon varying crystallization temperature; (b) Rate constant B1 and B2 plotted as a function of crystallization temperature;(c) Complex viscosity of precompacted molten sample discs composed of PLLA140 and PDLA90 as a function of time at $1 \mathrm{rad} / \mathrm{s}$ and different temperatures. 
In Figure 5.10(a), the storage modulus G' of precompacted discs of the L140-D90 blend is plotted as a function of time in the temperature range of 190 to $220^{\circ} \mathrm{C}$. For all crystallization temperatures, an initial high rate of $G$ ' buildup is followed by a reduced rate as observed previously. Again, the rate constant B1 and B2 for the two distinctive regimes are calculated and presented as a function of temperature in Figure 5.10(b). The rate constant B2 decreases slightly with the decrease in temperature, which is assigned to a cooling induced increase in viscosity and decreased chain diffusivity in the established network. Due to the relatively slow temperature ramp from 200 to $220^{\circ} \mathrm{C}$, prior to the start of the experiment, the first measurement point of the $220^{\circ} \mathrm{C}$ curve is omitted in the calculation of B1. Upon decreasing from 220 to $190^{\circ} \mathrm{C}$, the rate constant B1 initially increases and remains constant from $200^{\circ} \mathrm{C}$ onwards, confirming a balance between chain diffusivity and crystallization rate both as a function of temperature. At $220^{\circ} \mathrm{C}$ chain diffusivity is high, but the degree of supercooling as a driving force for crystallization is low, which is witnessed by the lower rate constant B1. It is worth noting that divergence is seen between the rheology (Figure 5.10) and DSC (Figure 5.9(b)). This could be a result of the different requirements in measuring modulus (network formation) against the enthalpy required for the melting of the crystals. Additionally, the high ultimate stiffening despite the slow initial stereocomplexation rate at $220^{\circ} \mathrm{C}$ may have been promoted by early stereocrystallization while heating to the ultimate measurement temperature in the rheometer.

The isothermal rheometry measurements in the temperature range of 190 to $220^{\circ} \mathrm{C}$, the crystalline morphology was investigated via POM and FTIR imaging. The results are illustrated in Figure 5.11. From the FTIR absorbance images (Figure 5.11(b)) made at different crystallization temperatures, the width of the stereocomplexation band (bandwidth) can be seen in Figure 5.12. Upon increasing the isothermal crystallization temperatures, the bandwidth and the stereo- to homocrystal ratio increase steadily until $210^{\circ} \mathrm{C}$. As the initial complex viscosity lowers with increasing temperature, more stereocrystals can be formed before reaching a critical network density (deflection). From 210 to $220^{\circ} \mathrm{C}$, no increase in bandwidth is observed. This further proves that the low complex viscosity, high diffusivity and simultaneously reduced stereocomplexation rate at $220^{\circ} \mathrm{C}$ leads to a large bandwidth. 

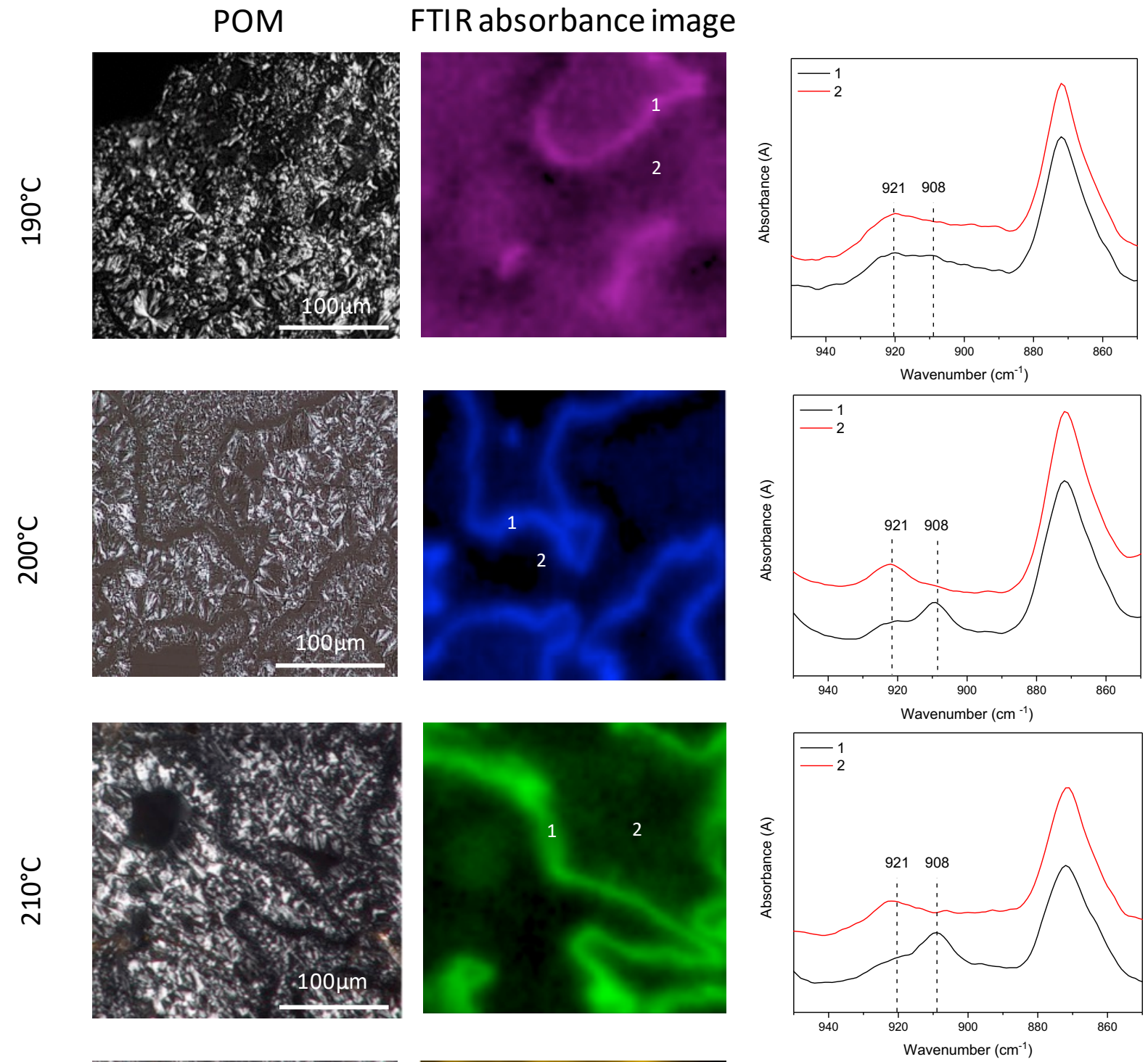

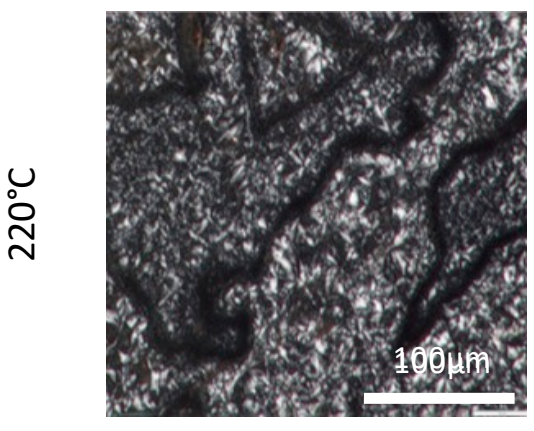

(a)

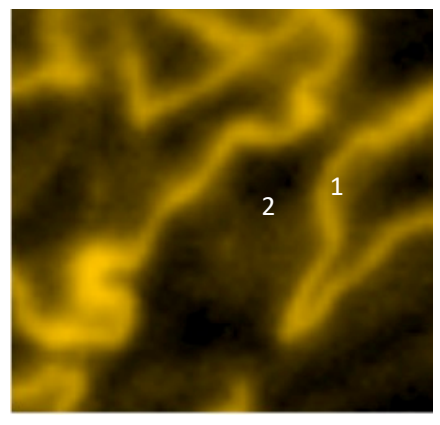

(b)

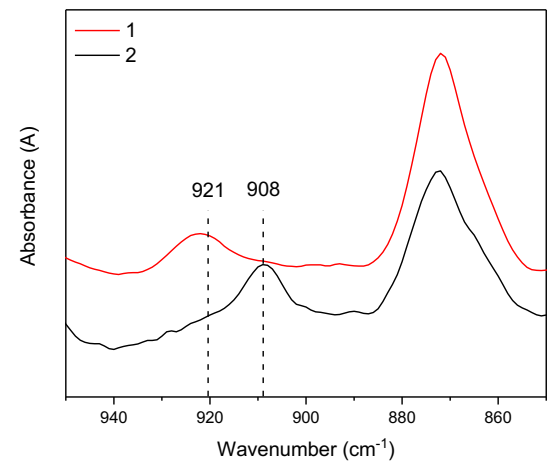

(c)

Figure 5.11: Interfacial crystalline morphology obtained after isothermal measurement at different temperatures via rheometry observed via (a) polarized optical microscopy (POM) and (b) corresponding FTIR absorbance image highlighting the interface produced. Note that the scale bars for POM and FTIR imaging are identical. (c) The absorbance spectra are plotted in region 1 (at the interface) and region 2 (aside the interface). The presence of stereocrystals is confirmed by the occurrence of characteristic $908 \mathrm{~cm}^{-1}$ band in region 1 and not in region 2. 


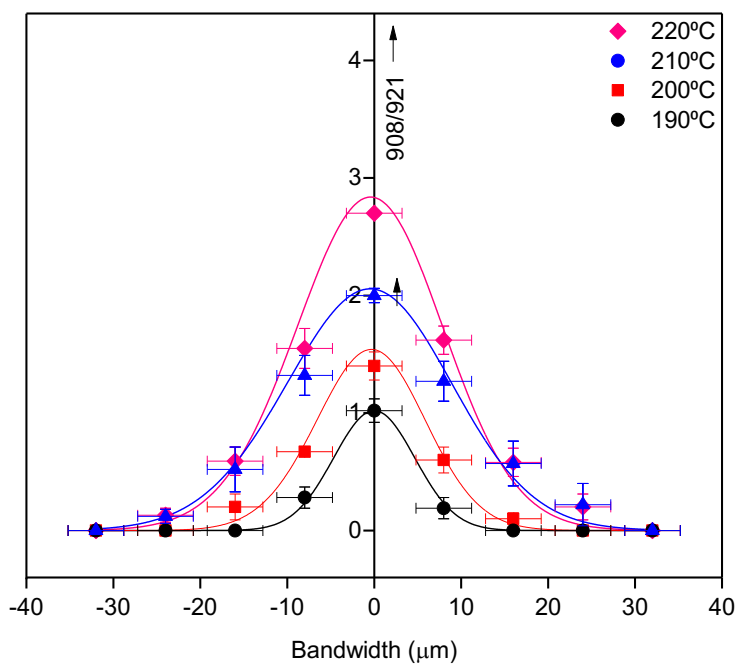

Figure 5.12: Bandwidth of stereocrystals calculated from FTIR imaging plotted as a function of crystallization temperature.

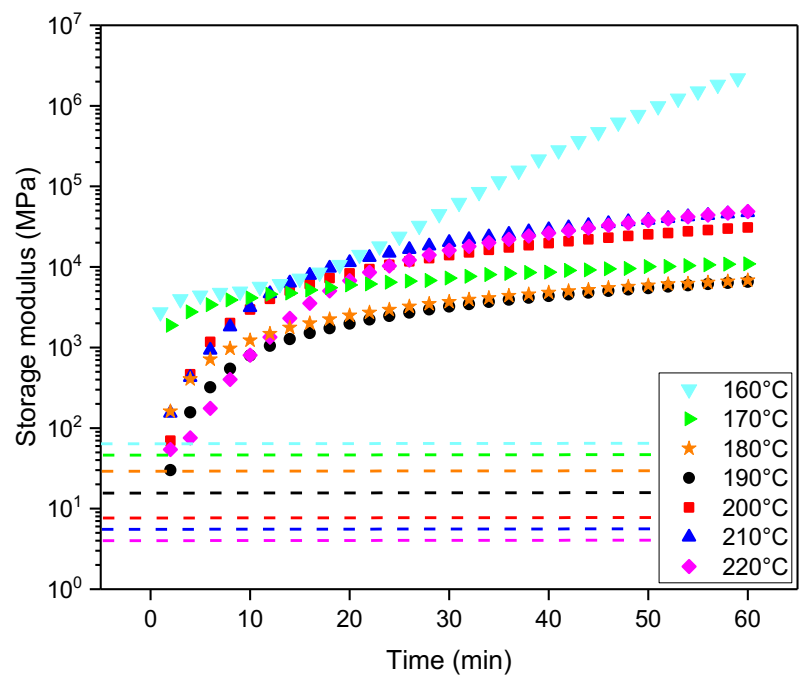

Figure 5.13: Storage modulus $\left(G^{\prime}\right)$ at $1 \mathrm{rad} / \mathrm{s}$ of precompacted molten disk made with opposite (dots) and identical (dashed) enantiomeric compositions as a function of time to probe the kinetics of interfacial stereo complexation upon varying crystallization temperature.

Lowering the temperature to the range of 180 to $160^{\circ} \mathrm{C}$, Figure 5.13 , reveals that the initial stage of stereocrystallization cannot be fully studied. However, it is worth mentioning that after 20 minutes at $160^{\circ} \mathrm{C}$ a distinct increase in the storage modulus $\mathrm{G}$ ' is observed. Being well above the conventional homocrystallization temperature ${ }^{25,37,38}$, the effect is attributed to stereocrystal induced homocrystallization. The combination of interfacial stiffening and 
successive nucleation is favorable in additive manufacturing technologies, aiding to mechanical reinforcement of weld regions and thermodynamic/geometrical stability that both limit the large scale adoption ${ }^{21}$. Here, the applicability in Selective Laser Sintering (SLS) is demonstrated.

\subsubsection{Stereocomplexation in selective laser sintering (SLS)}

SLS relies on the spatially controlled coalescence of powder particles via the use of a highintensity laser. The process takes place at elevated temperature, usually at a value close to the melting point, with a laser source sintering a layer of material by melting the powder interfaces. After the creation of a sintered layer, a layer of fresh powder is deposited on top and the process repeats until the part is completed ${ }^{39-42}$. The quality of sintering and the resulting mechanical properties are highly dependent on process parameters such as bed temperature, laser intensity or power, laser speed, and powder characteristics such as bulk density, powder flowability, particle shape, and particle size distribution. Evidently, the SLS process is non-isothermal in nature.

For this study, a 1:1 wt. ratio L140-D90 and L140-L90 blend with an average particle size of $100 \mu \mathrm{m}$ was prepared. Both blends were sintered into single layers, as described in the materials and methods section. To assess whether interfacial stereocrystallization occurs under SLS conditions, the amount of stereocrystals in single layer part was evaluated by DSC. $5 \mathrm{mg}$ of sample was heated from 30 to $250^{\circ} \mathrm{C}$ at a heating rate of $30^{\circ} \mathrm{C} / \mathrm{min}$ to prevent additional stereocrystallization upon heating. Figure 5.14 illustrates the respective DSC thermograms. No melting of stereocrystals is observed in the L140-L90 blend above $200^{\circ} \mathrm{C}$ as expected. However, for the L140-D90 blend, a small melting transition with an enthalpy of melting $\left(\Delta \mathrm{H}_{\mathrm{m}}\right)$ of $2.7 \mathrm{~J} / \mathrm{g}$ is clearly observed. In comparison to the reported enthalpies in Figure 5.8(a) and Figure 5.9(b) after one hour, the observed enthalpy after laser sintering is low, but cannot be attributed to limited interfacial diffusion solely. It is to be noted that in selective laser sintering only the surface of the polymer particles is molten. Additionally, must be pointed out that the amount of stereocomplex crystals seen in SLS is not much lower than those seen under isothermal conditions $\left(200^{\circ} \mathrm{C}\right)$ when the time the timescales of part (layer) buildup in SLS ( $\left.5 \mathrm{~min}\right)$ is compared to the same isothermal time, viz, $2.7 \mathrm{~J} / \mathrm{g}$ and $3 \mathrm{~J} / \mathrm{g}$. This confirms that interfacial stereocrystallization takes place under the set non-isothermal SLS conditions. Thus, the concept will be of importance to additive manufacturing technologies based on thermoplastics. 


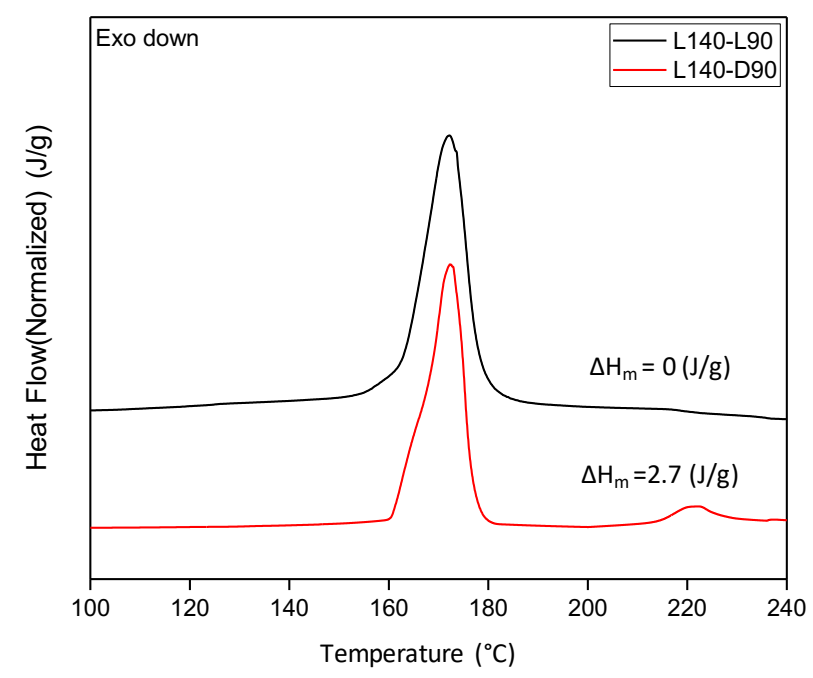

Figure 5.14: Normalized heat flow plotted as a function of temperature for samples prepared by selective laser sintering (SLS)

\subsection{CONCLUSIONS}

The kinetics and length-scales of isothermal interfacial stereocomplexation in heterogeneous poly(lactide) melts was studied by varying temperature and molar mass ratio of enantiomerically opposite poly(L-lactide) and poly(D-lactide) solid state pre-compacted samples where the molar mass of poly(D-lactide) was constant and the lowest overall. At $200^{\circ} \mathrm{C}$, which is well above the melting temperature of the homopolymeric poly(D-lactide) or poly(L-lactide) crystals and below the melting temperature of their stereocrystals, the stereocrystallization rate is inversely proportional to molar mass ratio as proven by the increase in storage modulus in rheometry. Interfacial stereocrystallization proceeds via two successive stages where the low molar mass poly(D-lactide) primarily diffuses into the higher molar mass poly(L-lactide) domains following reptation. After an initial steep increase in storage modulus, where the rate is molar mass dependent, a transition occurs to a state where the stereocomplexation rate is low and the continuation of stereocrystallization is independent of molar mass. The relative population of stereo- to homocrystals spatially derived by FTIR imaging, reveals that the transition from high to low stereocrystallization rate is not dictated by the physical presence of crystals at the interface, but a critical complex viscosity and network density in which chain motion impedes significantly. The cooperative effect of entanglements and stereocrystals excludes the effect of molar mass in the second stage of stereocrystallization. Stereocrystals require per definition multiple polymer chains that like entanglements act as physical crosslinks increasing cooperatively the storage modulus and complex viscosity of the heterogeneous melt. In the case of the lowest molar mass ratio, the number of entanglements per chain is the lowest, demanding 
the highest number of stereocrystals to reach the critical network density and facilitating the largest length-scales of interfacial stereocomplexation and ultimate mechanical stiffening.

The effect of temperature on interfacial stereocrystallization was studied for the lowest molar mass ratio marked by the highest interfacial diffusion rate, diffusion length-scales, total amount of stereocrystals and mechanical stiffening. The evolution of interfacial stereocrystallization by recording the melting enthalpy of stereocrystals in Differential Scanning Calorimetry compliments the rheometry studies. In comparison to homogenously mixed melts of two enantiomerically opposite poly(lactides), the rate of interfacial stereocrystallization in heterogeneous melts is initially high. Initial diffusivity and reeling in of chain segments towards crystal growth fronts of the low molar poly(D-lactide) is not or partially affected by the network density of the high molar mass poly(L-lactide). With variations in isothermal temperature, interfacial diffusion and successive stereocrystallization are no longer only dependent on relative viscosity, but also the thermodynamic driving force for crystallization, i.e. the degree of supercooling that both increase with a decrease in temperature. The length-scales of interfacial stereocrystallization and ultimate mechanical stiffening are based on a delicate balance of complex viscosity and the rate of stereocrystallization. At low supercooling, relatively low complex viscosity and stereocrystallization rate entail large timescales for reaching a critical network density and large ultimate diffusion paths and interfacial stiffening. As the isothermal temperature decreases, the degree of supercooling, the stereocrystallization rate and initial relative viscosities increase, reducing diffusivity, the timescales for critical network formation, and ultimate interfacial stiffening. At lower temperatures, but still above the conventional isothermal crystallization temperature of enantiomerically pure poly(lactides), stereocrystal induced poly(lactide) homocrystallization was observed. The judicious selection of relative molar masses and temperature creates a scenario in which (i) low individual homocrystallization rates that enable interfacial diffusion, (ii) successive high stereocrystallization rate and (iii) ultimate nucleation of homocrystals render technical advances in strengthening polymer-polymer interfaces during non-isothermal processing in for example additive manufacturing techniques like selective laser sintering. 


\section{REFERENCES}

(1) Quan, H.; Li, Z.-M.; Yang, M.-B.; Huang, R., On Transcrystallinity in Semi-Crystalline Polymer Composites. Composites Science and Technology. 2005, 65 (7-8), 999-1021.

(2) Leong, Y. W.; Yamaguchi, S.; Mizoguchi, M.; Hamada, H.; Ishiaku, U. S.; Tsujii, T., The Effect of Molding Conditions on Mechanical and Morphological Properties at the Interface of Film Insert Injection Molded Polypropylene-Film/Polypropylene Matrix. Polymer Engineering and Science. 2004, 44 (12), 2327-2334.

(3) Zhang, Z.; Xu, Q.; Chen, Z.; Yue, J., Nanohybrid Shish-Kebabs: Supercritical $\mathrm{CO}_{2}$-Induced PE Epitaxy on Carbon Nanotubes. Macromolecules. 2008, 41 (8), 2868-2873.

(4) Li, C. Y.; Li, L.; Cai, W.; Kodjie, S. L.; Tenneti, K. K., Nanohybrid Shish-Kebabs: Periodically Functionalized Carbon Nanotubes. Advanced Materials. 2005, 17 (9), 1198-1202.

(5) Ning, N.; Fu, S.; Zhang, W.; Chen, F.; Wang, K.; Deng, H.; Zhang, Q.; Fu, Q., Realizing the Enhancement of Interfacial Interaction in Semicrystalline Polymer/Filler Composites via Interfacial Crystallization. Progress in Polymer Science. 2012, 37 (10), 1425-1455.

(6) Ishida, H.; Bussi, P., Surface-Induced Crystallization in Ultrahigh-Modulus Polyethylene Fiber Reinforced Polyethylene Composites. Macromolecules. 1991, 24 (12), 3569-3577.

(7) Nyambo, C.; Mohanty, A. K.; Misra, M., Polylactide-Based Renewable Green Composites from Agricultural Residues and Their Hybrids. Biomacromolecules. 2010, 11 (6), 1654-1660.

(8) Magniez, K.; Voda, A. S.; Kafi, A. A.; Fichini, A.; Guo, Q.; Fox, B. L., Overcoming Interfacial Affinity Issues in Natural Fiber Reinforced Polylactide Biocomposites by Surface Adsorption of Amphiphilic Block Copolymers. ACS Applied Materials and Interfaces. 2013, 5 (2), 276-283.

(9) Xu, H.; Xie, L.; Chen, Y.-H.; Huang, H.-D.; Xu, J.-Z.; Zhong, G.-J.; Hsiao, B. S.; Li, Z.-M., Strong Shear Flow-Driven Simultaneous Formation of Classic Shish-Kebab, Hybrid Shish-Kebab, and Transcrystallinity in Poly(Lactic Acid)/Natural Fiber Biocomposites. ACS Sustainable Chemistry and Engineering. 2013, 1 (12), 1619-1629.

(10) Wang, Y.; Tong, B.; Hou, S.; Li, M.; Shen, C., Transcrystallization Behavior at the Poly(Lactic Acid)/Sisal Fibre Biocomposite Interface. Composites Part A: Applied Science and Manufacturing. 2011, $42(1), 66-74$.

(11) Ikada, Y.; Jamshidi, K.; Tsuji, H.; Hyon, S. H., Stereocomplex Formation between Enantiomeric Poly(Lactides). Macromolecules. 1987, 20 (4), 904-906.

(12) Lee, S.; Kimoto, M.; Tanaka, M.; Tsuji, H.; Nishino, T., Crystal Modulus of Poly (Lactic Acid)s, and Their Stereocomplex. Polymer. 2018, 138, 124-131.

(13) Tsuji, H. Poly(Lactic Acid) Stereocomplexes: A Decade of Progress. Advanced Drug Delivery Reviews. 2016, 107, 97-135.

(14) Habibi, Y.; Aouadi, S.; Raquez, J. M.; Dubois, P., Effects of Interfacial Stereocomplexation in Cellulose Nanocrystal-Filled Polylactide Nanocomposites. Cellulose, 2013, 20 (6), 2877-2885.

(15) Ma, P.; Lv, P.; Xu, P.; Du, M.; Zhu, H.; Dong, W.; Chen, M., Design of Bio-Based Conductive and Fast Crystallizing Nanocomposites with Controllable Distribution of Multiwalled Carbon Nanotubes via Interfacial Stereocomplexation. Chemical Engineering Journal. 2018, 336, 223-232.

(16) Arias, V.; Odelius, K.; Höglund, A.; Albertsson, A.-C., Homocomposites of Polylactide (PLA) with Induced Interfacial Stereocomplex Crystallites. ACS Sustainable Chemistry and Engineering. 2015, 3 (9), 2220-2231.

(17) Wang, B.; Wen, T.; Zhang, X.; Tercjak, A.; Dong, X.; Müller, A. J.; Wang, D.; Cavallo, D., Nucleation of Poly(Lactide) on the Surface of Different Fibers. Macromolecules. 2019, 52 (16), 6274-6284.

(18) Wen, T.; Xiong, Z.; Liu, G.; Zhang, X.; De Vos, S.; Wang, R.; Joziasse, C. A. P.; Wang, F.; Wang, D., The Inexistence of Epitaxial Relationship between Stereocomplex and a Crystal of Poly(Lactic Acid): Direct Experimental Evidence. Polymer. 2013, 54 (7), 1923-1929.

(19) Chen, R.; Huang, Y.-H.; Liu, L.; Chen, L.-B.; Liu, Z.-Y.; Yang, W.; Yang, M.-B., Diverse Interfacial Crystalline Morphologies Induced by Poly (d-Lactide) (PDLA) Melt Penetration Process in Multi-Melt Multi-Injection Molding (M3IM) System. Composites Part B: Engineering. 2018, 153, 429-436.

(20) Akagi, T.; Fujiwara, T.; Akashi, M., Rapid Fabrication of Polylactide Stereocomplex Using Layerby-Layer Deposition by Inkjet Printing. Angewandte Chemie International Edition. 2012, 51 (22), 5493-5496.

(21) Srinivas, V.; van Hooy-Corstjens, C. S. J.; Vaughan, G. B. M.; van Leeuwen, B.; Rastogi, S.; Harings, J. A. W., Interfacial Stereocomplexation To Strengthen Fused Deposition Modeled Poly(Lactide) Welds. ACS Applied Polymer Materials. 2019, 1 (8), 2131-2139.

(22) Bousmina, M.; Qiu, H.; Grmela, M.; Klemberg-Sapieha, J. E., Diffusion at Polymer/Polymer Interfaces Probed by Rheological Tools. Macromolecules. 1998, 31 (23), 8273-8280.

(23) Zachmann, V. H. G.; Stuart, H. A. Schmelz- Und Kristallisationserscheinungen Bei 
Makromolekularen Substanzen. IV. Haupt- Und Nachkristallisation von Terylen Aus Dem Glaszustand. Die Makromolekulare Chemie. 1960, 41 (1), 131-147.

(24) Brochard, F.; Jouffroy, J.; Levinson, P., Polymer-Polymer Diffusion in Melts. Macromolecules. 1983, 16 (10), 1638-1641.

(25) Srinivas, V.; van Hooy-Corstjens, C. S. J.; Harings, J. A. W., Correlating Molecular and Crystallization Dynamics to Macroscopic Fusion and Thermodynamic Stability in Fused Deposition Modeling; a Model Study on Polylactides. Polymer. 2018, 142, 348-355.

(26) Pan, P.; Yang, J.; Shan, G.; Bao, Y.; Weng, Z.; Cao, A.; Yazawa, K.; Inoue, Y., TemperatureVariable FTIR and Solid-State ${ }^{13}$ C NMR Investigations on Crystalline Structure and Molecular Dynamics of Polymorphic Poly( I -Lactide) and Poly( I -Lactide)/Poly( d -Lactide) Stereocomplex. Macromolecules. 2012, 45 (1), 189-197.

(27) Zhang, J.; Tashiro, K.; Tsuji, H.; Domb, A. J., Disorder-to-Order Phase Transition and Multiple Melting Behavior of Poly(l-Lactide) Investigated by Simultaneous Measurements of WAXD and DSC. Macromolecules. 2008, 41 (4), 1352-1357.

(28) Zhang, J.; Duan, Y.; Sato, H.; Tsuji, H.; Noda, I.; Yan, S.; Ozaki, Y., Crystal Modifications and Thermal Behavior of Poly(L-Lactic Acid) Revealed by Infrared Spectroscopy. Macromolecules. 2005, 38 (19), 8012-8021.

(29) Tsuji, H.; Bouapao, L., Stereocomplex Formation between Poly(L-Lactic Acid) and Poly(D-Lactic Acid) with Disproportionately Low and High Molecular Weights from the Melt. Polymer International. 2012, 61 (3), 442-450.

(30) Pan, P.; Han, L.; Bao, J.; Xie, Q.; Shan, G.; Bao, Y., Competitive Stereocomplexation, Homocrystallization, and Polymorphic Crystalline Transition in Poly(L-Lactic Acid)/Poly(D-Lactic Acid) Racemic Blends: Molecular Weight Effects. Journal of Physical Chemistry. 2015, 119 (21), 6462-6470.

(31) Saeidlou, S.; Huneault, M. A.; Li, H.; Park, C. B., Poly(Lactic Acid) Stereocomplex Formation: Application to PLA Rheological Property Modification. Journal of Applied Polymer Science. 2014, 131 (22).

(32) Wei, X.-F.; Bao, R.-Y.; Cao, Z.-Q.; Yang, W.; Xie, B.-H.; Yang, M.-B., Stereocomplex Crystallite Network in Asymmetric PLLA/PDLA Blends: Formation, Structure, and Confining Effect on the Crystallization Rate of Homocrystallites. Macromolecules. 2014, 47 (4), 1439-1448.

(33) Kawai, T.; Rahman, N.; Matsuba, G.; Nishida, K.; Kanaya, T.; Nakano, M.; Okamoto, H.; Kawada, J.; Usuki, A.; Honma, N., Crystallization and Melting Behavior of Poly (L-Lactic Acid). Macromolecules. 2007, 40 (26), 9463-9469.

(34) Piorkowska, E.; Rutledge, G., Handbook of Polymer Crystallization, 2013, ISBN 9780470380239. .

(35) Yasuniwa, M.; Tsubakihara, S.; lura, K.; Ono, Y.; Dan, Y.; Takahashi, K., Crystallization Behavior of Poly(l-Lactic Acid). Polymer. 2006, 47 (21), 7554-7563.

(36) Brumer, P.,Advances in Chemical Physics; John Wiley \& Sons, Inc: Hoboken, NJ, USA, 2016; Vol. 159, ISBN 9781119096269.

(37) Li, H.; Huneault, M. A., Effect of Nucleation and Plasticization on the Crystallization of Poly(Lactic Acid). Polymer. 2007, 48 (23), 6855-6866.

(38) Saeidlou, S.; Huneault, M. A.; Li, H.; Park, C. B., Poly(Lactic Acid) Crystallization. Progress in Polymer Science. 2012, 37 (12), 1657-1677.

(39) Williams, J. D.; Deckard, C. R., Advances in Modeling the Effects of Selected Parameters on the SLS Process. Rapid Prototyping Journal. 1998, 4 (2), 90-100.

(40) Wendel, B.; Rietzel, D.; Kühnlein, F.; Feulner, R.; Hülder, G.; Schmachtenberg, E., Additive Processing of Polymers. Macromolecular Materials and Engineering. 2008, 293 (10), 799-809.

(41) Drummer, D.; Rietzel, D.; Kühnlein, F., Development of a Characterization Approach for the Sintering Behavior of New Thermoplastics for Selective Laser Sintering. Physics Procedia. 2010, 5, 533-542.

(42) Gebhardt, A.; Understanding Additive Manufacturing. 2011, I-IX, ISBN 9783446425521. 

Chapter 6

Valorization; translation of the know-how for the development of products for manufacturing and biomedical industry 


\subsection{TECHNOLOGICAL IMPACT IN THE MANUFACTURING INDUSTRY}

In this thesis, the problem of inferior inter-layer weld mechanics observed in additively manufactured thermoplastic parts, specifically realized by FDM, is addressed. In Chapter 2, the importance of misaligned timescales of intermolecular diffusion and crystallization is highlighted by systematic variations in molecular parameters using poly(lactides). The subsequent chapters demonstrate the enhancement of inter-layer weld stiffness by controlling molecular diffusion and /or crystallization. In Chapter 3, the addition of chemically identical low molar mass fractions and nucleating poly(lactide) stereocomplex crystals provide melt plasticization and increased crystallization rate, leading to an increase in weld stiffness and geometrical stability. A more effective route to simultaneously increase weld stiffness and enhance geometrical stability is provided and investigated in Chapter 4. The innovative fabrication strategy relies on weld reinforcement via spatially targeting stereocomplexation across layer interfaces. This is achieved by alternating deposition of enantiomerically opposite poly(lactides). Furthermore, the kinetics, spatial distribution and length scales of interfacial stereocrystallization under isothermal and non-isothermal conditions are addressed in detail in Chapters 4 and 5.

Poly(lactides) have been and are today still the primary class of thermoplastic used in FDM and has been successfully adopted in manufacturing industries to make functional prototypes, rapid tooling, and customized parts ${ }^{1-4}$. In many applications, the intrinsic mechanical properties are expected and desired. However, due to the slow molecular dynamics and morphological changes of the semi-crystalline polymer with time, a mismatch in the anticipated and the experimentally realized mechanical properties exists. The routes of blending low molar mass additives and interfacial stereocomplexation presented in this thesis are a unique and effective way to enhance inter-layer mechanical stiffness. Moreover, for the incorporation of these concepts in the 3D printing industry, no major modifications are required in existing FDM printing setups. To utilize low molar mass additives, the blends can be fed in the form of filaments or granules (feedstock for FDM printers). Achieving interfacial stereocomplexation in FDM requires a twin nozzle setup, which is embedded in most FDM printers today. However, it is important to note that in all printers a second nozzle is typically provided for deposition of a support material, which aids in the formation of complex geometries. The support material is typically watersoluble and is removed after printing of the object. Therefore, the printing of complicated geometries in combination with interfacial stereocomplexation demands the use of multimaterial FDM printers having more than two nozzles.

As highlighted in Chapter 5, interfacial stereocomplexation has great potential in Selective Laser Sintering (SLS), where the interfacial area is much greater than in FDM. Future research and 
development on interfacial stereocrystallization in SLS is required, which shall focus on process optimization and evaluation of mechanical properties. It is also worth noting that stereocomplexation phenomena is not limited to poly(lactides). Complementary configurations that under specific conditions lead to stereocrystallization have been reported for various polymer systems such as aliphatic polyesters, aliphatic polycarbonates, polyimides, polyketones, and poly(methyl methacrylate $)^{5-8}$. Consequently, interfacial stereocomplexation to mechanically reinforce polymer-polymer interfaces by effective stress transfer is applicable to other polymeric materials, expanding the diversity in mechanical properties, and certainly not limited to additive manufacturing. Composites comprise a class of materials in which a dispersed discontinuous phase adds functionality to the continuous matrix phase. Mechanical reinforcement of the dispersed phase to the continuous phase is always a challenging step in perceiving the ultimate properties of a composite. Thus, the interfacial stress transfer is often challenged but can be secured via the concept of interfacial stereocrystallization.

Apart from applications in the manufacturing industry, Poly(lactides) are commonly used in biomedical applications as it uniquely combines biodegradability, biocompatibility, and thermoplastic facile processability ${ }^{9,10}$. The use of poly(lactide) is not solely based on its biodegradability nor because it is made from renewable resources. It is being used because in comparison to competitively classified polymers as it performs very well and provides excellent properties at a low price ${ }^{11}$. Via FDM technology, poly(lactide) in the biomedical industry is primarily utilized in the fabrication of bioresorbable implants and porous scaffolds for tissue regeneration. In the forthcoming section, the fundamental knowledge originating from this thesis is exploited by the introduction of an innovative fabricate route of porous scaffolds for tissue engineering with enhanced biomedical functionality.

\subsection{TECHNOLOGICAL IMPACT IN TISSUE ENGINEERING}

\subsubsection{Introduction}

Evolution has provided nature with myriads of exquisitely engineered load-bearing constructs. To facilitate energetically optimized operations, the functional endurance of natural loadbearing materials is based on porous, fibrillar and anisotropically reinforced composite structures fulfilling the requirement of being lightweight. The closest example is the building block of our skeleton. Bone encompasses a class of materials mainly consisting of mineralized collagen fibrils. To fulfill the requirement of mechanical support, uniquely spatially organized multi-sized pores are desired ${ }^{12}$. Due to the presence of bone marrow, bone is a vascularized tissue that besides collagen contains various structural proteins. To render smoothness, i.e. low friction, transfer of loads in the cooperative functioning of the skeleton, the end of bones known as the osteochondral interface - are made of cartilage, a special connective tissue (as -110 - 
seen in Figure 6.1). Whereas bone is vascularized, cartilage is not, leading to a low regeneration potential after damage ${ }^{13,14}$. Damage of this cartilage may lead to osteoarthritis, which is a chronic, progressive and degenerative disease that not only affects the elderly but also sportive younger athletes with joints that are excessively stressed ${ }^{15}$. Studies of the United Nations predict that by 2050 about 130 million people will suffer from osteoarthritis ${ }^{16}$.

Besides initial pain medication, the treatment of, for example, knee cartilage lesions and symptoms are challenged by its avascular nature. Consequently, cartilage possesses low healing capacity that ultimately invokes joint replacement and even total knee arthroplasty ${ }^{17,18}$. The performance of the currently used knee prostheses is unideal since its life-time is (i) limited to 10-15 years, demands revision surgeries, and (ii), if it is mechanically overdesigned, weakens the resulting cooperative mechanical functionality. In terms of mechanical overdesign in reinstigating anisotropic bone mechanics, it is remarkable to realize that most implants today, also the ones that are temporary and bio-resorbable in origin and are mechanically isotropically designed. Such a mismatch in mechanical design has adverse effects on the functioning of the regenerated or repaired tissue.

Biofabrication to direct tissue regeneration of the defected osteochondral tissue seems most promising. Here, cells are seeded and are grown on an either in-vitro or in-vivo geometrically shaped scaffold that slowly degrades. Timing of degradation, and thus fading of the functionalities like shape and mechanics, must be aligned with the timescales of growth and self-functioning of the tissue. Due to ease and flexibility in shaping, thermoplastic polymers are preferred as a scaffold material. FDM is often used to geometrically reproduce the defected tissue by constructing scaffolds. An empirically derived rule of thumb for the best regeneration is accomplished using materials, here scaffolds, that mimic the functionalities of the native tissue as close as possible ${ }^{19}$. Known functionalities are surface roughness, surface chemistry, porosity, surface - and macroscopic mechanics, often non-isotopically distributed in the native gradient tissue ${ }^{20-24}$. Each parameter influences cell attachment, proliferation, and differentiation.

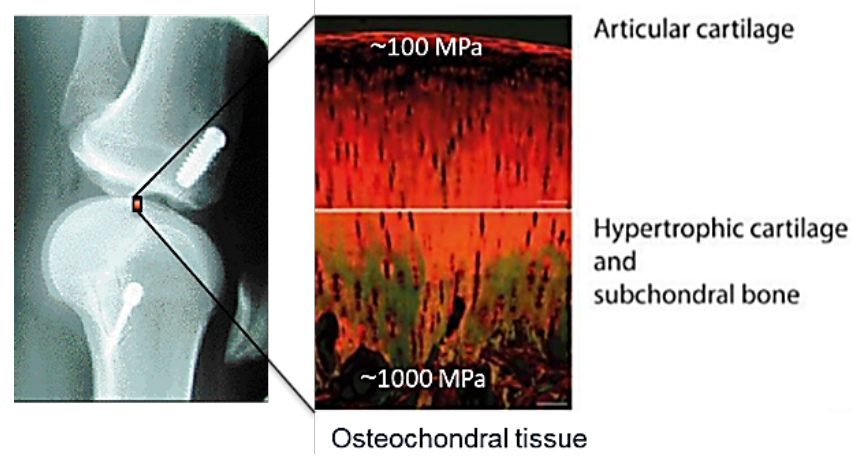

Figure 6. 1: Fixation of regenerative tissue using a mechanically isotropic screw (left) and deeper insight in the mechanical gradient of the osteochondral tissue (right), processed from Di Luca et al. ${ }^{24}$. 
The effect of gradient porosity on the differentiation of human mesenchymal stromal cells (hMSCs) was studied by Di Luca et al. ${ }^{25}$. In the study, 3D printed scaffolds of poly(ethylene oxide terephthalate)/ poly(butylene terephthalate) (PEOT/PBT) with gradient porosity, homogenous surface chemistry and surface mechanics were used. Along a gradient in descending pore size, a gradual increase in chondrogenic markers and compaction of the formed extracellular matrix were observed. To reach ultimate control in hMSCs differentiation lineage, attempts are made to complement gradient porosity by gradient surface mechanics- a concept proven for the regeneration of soft tissue ${ }^{26}$. The existence of such a stiffness - cell differentiation relationship for stiff tissue regeneration is yet a matter of debate and requires investigation. Two approaches to introduce spatial gradient (surface) mechanics are based on (i) either discrete mechanical transitions or, (ii) continuous transition/gradient in mechanics ${ }^{24,27}$. Practically, both approaches are based on variations in materials and consequently materials chemistry, inducing an additional effect in terms of biological response and cell attachment ${ }^{28}$. On top of that, multimaterial scaffolds with discrete mechanical transitions are prone to macroscopically fail once cooperatively mechanically stressed.

To optimize the targeted functionality of regenerated tissue (here the osteochondral tissue), it may be beneficial to preserve homogenous surface chemistry for cell attachment and introduce natural gradients in macro-mechanics via a single component FDM printed system. The stiffness of a single bioresorbable polymer can be spatially controlled by directing the degree of crystallinity in three-dimensional space. The gradient structures developed can be used as directly implantable bioresorbable support structures and scaffolds in regenerative tissue engineering. To achieve and direct crystallinity gradients, the observations made in Chapter 2 are recalled. Thermally induced structural heterogeneities along the build direction can evolve in parts printed with PLA due to the low crystallizing rate of PLA. In addition, to achieve a gradient structure, it is also important that (a) the range of stiffness closely mimics the stiffness range of osteochondral interfaces and, (b) the parameters that influence cell differentiation such as surface chemistry and porosity are not altered. In the following section, the fabrication of stiffness gradients by controlled variations in crystallinity via FDM printing is explored. The achievable gradients in stiffness are demonstrated by employing DMA and mechanical indentation studies.

\subsubsection{Materials and methods}

\subsubsection{Material selection}

A biomedical PLLA grade with an enantiomeric purity above $99 \%$ and an inherent viscosity of $1.8 \mathrm{dl} / \mathrm{g}$ was provided by Corbion Purac (Gorinchem, The Netherlands). Since the glass transition temperature $\left(T_{g}\right)$ of this material is $58^{\circ} \mathrm{C}$, the material stays above its $T_{g}$ at body temperature 
$\left(\sim 37^{\circ} \mathrm{C}\right)$, rendering only slight variations in crystallinity induced stiffness. The optimum design of a scaffold dictates a high gradient in stiffness, as it is reported that the osteochondral interface comprises of a stiff subchondral bone and significantly softer mineralized cartilage. Here, the stiffness can vary within a range of 100 to above $1000 \mathrm{MPa}{ }^{24,25,29,30}$. To achieve stiffness variation in this order of magnitude, the $T_{g}$ of PLLA needs to be reduced significantly, so that stiffness variations due to crystallinity are more expressive and fit the targeted range in stiffness. However, the $T_{g}$ cannot be much below body temperature, as it will lead to cold crystallization overtime, erasing the structural variations and inducing undesired dimension instability. To reduce the $T_{g}$, vitamin $E$ (a-tocopherol) was used as a plasticizer. This selection was based on the fact that Vitamin $E$ is (a) biocompatible and potentially bioactive ${ }^{31}$, and (b) relatively hydrophobic ${ }^{32,33}$, reducing the probability of leeching out and causing an uncontrolled increase in $T_{\mathrm{g}}$ over time. A series of PLLA-Vitamin $\mathrm{E}$ blends were produced by solution mixing PLLA and Vitamin $E$ in chloroform for 1 hour under continuous stirring. The mixture was then poured into a petri dish and chloroform was allowed to evaporate at ambient temperature. Subsequently, the $\mathrm{T}_{\mathrm{g}}$ of the blend was determined by Differential Scanning Calorimetry (DSC), by heating the sample from 0 to $200^{\circ} \mathrm{C}$ at a heating rate of $10^{\circ} \mathrm{C} / \mathrm{min}$. In Figure 6.2 , the $\mathrm{T}_{\mathrm{g}}$ of PLLA is followed as a function of vitamin E concentration by weight in the mixture. It is apparent that the $T_{g}$ decreases with the increasing wt-\% of vitamin $E$. The addition of $18 \mathrm{wt}$. $\%$ of vitamin $\mathrm{E}$ resulted in a $\mathrm{T}_{\mathrm{g}}$ close to $37^{\circ} \mathrm{C}$ and was used for further studies. From here onwards, in this chapter, this blend will be referred to as PLLA-V18.

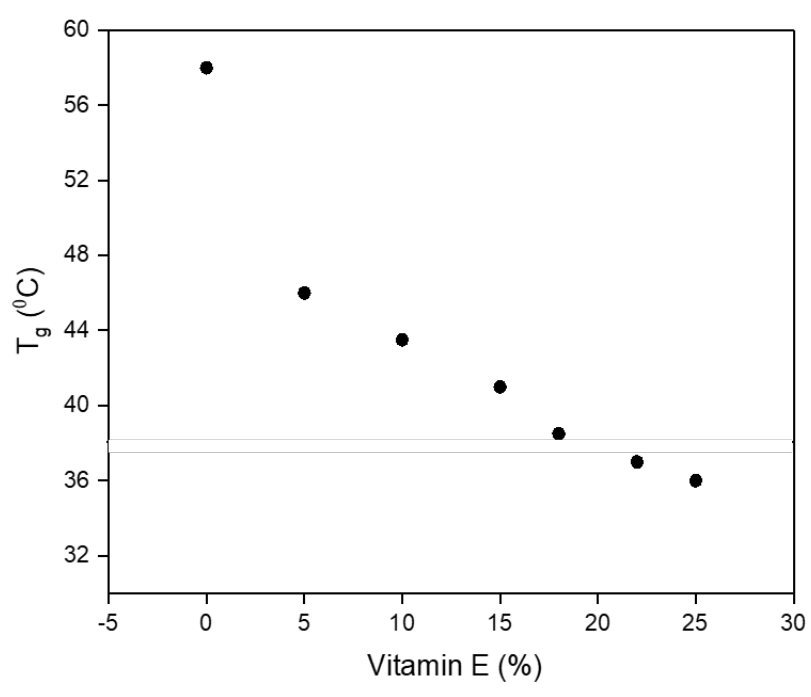

Figure 6.2: Glass transition temperature $\left(T_{g}\right)$ of PLLA plotted as a function of the concentration of vitamin $E$ measured by weight. 


\subsubsection{Scaffold design}

For scaffold fabrication, a PLLA-V18 filament with a mean diameter of $2.85 \mathrm{~mm}$ was prepared by melt extrusion at $190^{\circ} \mathrm{C}$ using a 3Devo single screw extruder. Thereafter, scaffolds were fabricated using an Ultimaker $2+$ filament printer operating at a melt temperature of $190^{\circ} \mathrm{C}$ and a build plate temperature of $60^{\circ} \mathrm{C}$. The sample having 18 layers, with a layer height of $0.4 \mathrm{~mm}$, an infill of $50 \%$, a $0 / 90^{\circ}$ deposition strategy was printed. The dimensions of the scaffold were set to $20 \times 20 \times 7.2 \mathrm{~mm}^{3}$ as illustrated in Figure 6.3. To attain a crystallinity gradient along the build direction, where the bottom layer would have the highest crystallinity, the printing speed was set such that the time taken to attain maximum crystallinity was within the time taken to construct the part. Based on the isothermal crystallization measurement at $60^{\circ} \mathrm{C}$ of PLLA-V18, the time taken to achieve maximum crystallinity in the bottom layer was estimated. It is also important to note that in the current printing setup, a heated bottom plate controls the ambient air temperature. Due to the absence of a heated chamber, the ambient temperature decreases a function of build height and is not constant. This may further aid in achieving a crystallinity gradient, provided heat is sufficiently dissipated during deposition. To confirm this temperature profiling of the bottom layer was also performed.

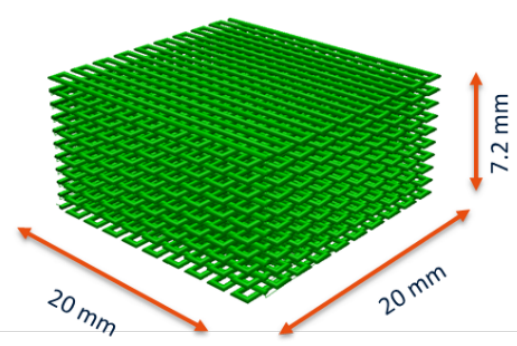

Figure 6.3: Schematic of sample geometry.

\subsubsection{Temperature recording}

To understand and control the evolution of a gradient in crystallinity along the build direction, the temperature history of the first deposited layer was mapped with varying time intervals between the deposition of successive layers. Temperature profiles were recorded using a 0.15 $\mathrm{mm} \mathrm{K}$ type thermocouple embedded in the base plate of the printer. The thermocouple was placed in the middle of the bottom layer. High accuracy in data acquisition was guaranteed by a DataQ DI718 data acquisition read-out system with $1 \mathrm{kHz}$ sampling frequency.

\subsubsection{Structural analysis in scaffold}

The crystallinity of individual layers was determined via 2D wide-angle $X$-ray diffraction using a SAXSLAB's Ganesha instrument equipped with a divergence source producing X-ray photons with 
a wavelength of $1.54 \mathrm{~A}^{\circ}$. The beam center and $\theta$ range were calibrated using the diffraction peaks of silver behenate. Conversion of 2D into 1D data was carried out with Saxsgui V2.13.01 using an automated beam stop mask.

\subsubsection{Isothermal crystallization behaviour of PLLA-V18}

Differential Scanning Calorimetry (DSC) was used to study the crystallization behaviour of the PLLA-V18 blend. For the study, an amorphous sample was prepared by melting the solution blended sample between two silicon wafers at $200^{\circ} \mathrm{C}$ and subsequently quenching in a dry ice acetone mixture of $-78^{\circ} \mathrm{C}$. DSC was performed using a TA instruments Q2000 by weighing $5 \mathrm{mg}$ of sample with $\pm 5 \%$ accuracy in an aluminium pan that was sealed hermetically. The sample was heated at a rate of $30^{\circ} \mathrm{C} / \mathrm{min}$ to a temperature of $60^{\circ} \mathrm{C}$ and kept isothermally for one hour to ensure (cold) crystallization. The relative crystallinity $\left(X_{t}\right)$ is plotted as a function of time in Figure 6.4. It is observed that maximum crystallinity $\left(X_{t}=1\right)$ is achieved within approximately 8 minutes.

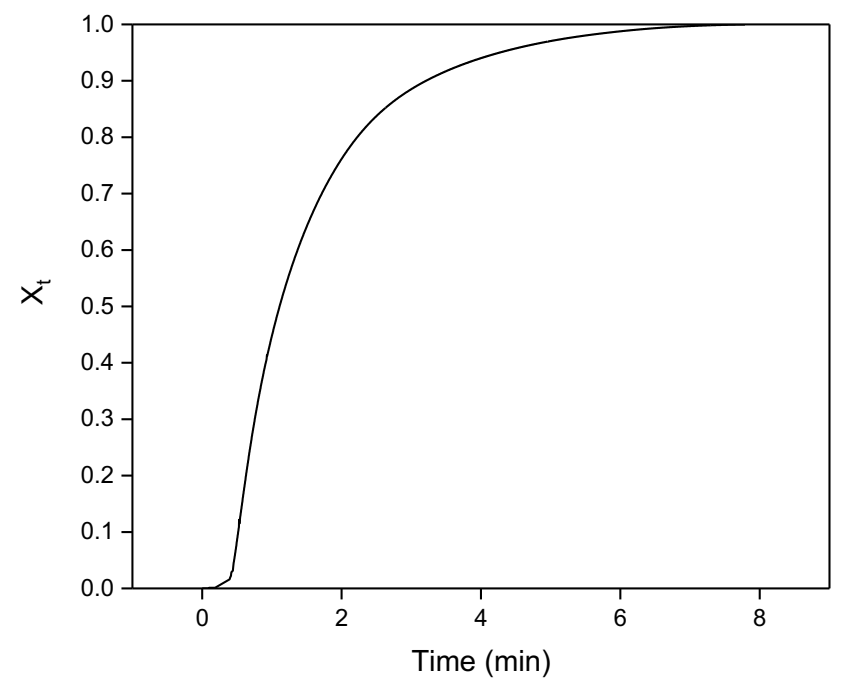

Figure 6.4: Relative crystallinity $\left(X_{t}\right)$ plotted a function of time for isothermal crystallization of PLLAV18 performed at $60^{\circ} \mathrm{C}$.

\subsubsection{Mechanical characterization}

The elastic modulus (E') was evaluated by dynamic mechanical testing in tension mode using a Mettler Toledo DMA 1. The deformation was set to $5 \mu \mathrm{m}$ and at a frequency of $1 \mathrm{~Hz}$. The storage modulus ( $E^{\prime}$ ) was recorded in a temperature range from 25 to $100{ }^{\circ} \mathrm{C}$ using a heating rate of 5 ${ }^{\circ} \mathrm{C} / \mathrm{min}$. The shear modulus ( $G$ ') was measured by dynamic mechanical testing in torsion mode using an Anton Parr MCR 302 at a strain of $0.01 \%$ and a frequency of $1 \mathrm{~Hz}$.

To evaluate the surface stiffness, indentation studies were performed on a Nanolndenter XP (MTS Systems, USA). Experiments were performed in controlled displacement mode using $3 \mu \mathrm{m}$ displacement and a $2 \mathrm{~mm}$ cylindrical flat-ended indenter. During the measurement, the force 
as a function of displacement was recorded during the loading and unloading cycle. A total of five measurements were performed on various locations on the sample.

\subsubsection{Results and discussion}

\subsubsection{Stiffness gradient}

To confirm the hypothesis that the chosen plasticized PLLA system (PLLA-V18) can provide a significant gradient in stiffness, the mechanical properties were investigated in the amorphous and crystalline state. Films of solution blended PLLA-V18 (section 6.2.2.5) were heated to $190^{\circ} \mathrm{C}$ between two silicon wafers and kept isothermally for 2 minutes to erase the thermal history. Thereafter, samples were quenched in acetone to circumvent crystallization and achieve an amorphous state $\left(X_{t}=0\right)$. In order to achieve samples with maximum crystallinity $\left(X_{t}=1\right)$, the prepared amorphous samples were crystallized under isothermal conditions in a preheated oven at $60^{\circ} \mathrm{C}$ for $8 \mathrm{~min}$, based on Figure 6.4. The thermo-mechanical properties of films with $X_{t}=0$ and $X_{t}=1$ were evaluated by DMTA under tension and torsion modes, to provide the E' and G', Figure 6.5 (a) and (b) respectively. As typically seen in thermoplastics, upon heating the glass transition induces a drop in storage modulus $E^{\prime}$ for both $X_{t}=1$ and 0 , Figure 6.5(a). Since polymer crystals contribute to network formation, the decrease in E' is more pronounced in the amorphous sample $\left(X_{t}=0\right)$. $X_{t}=1$ shows a higher stiffness compared to $X_{t}=0$ in the entire measured temperature range. Additionally, upon heating $X_{t}=0$ to higher temperatures, the decrease in E' is followed by an increase. The increase in E' is assigned to cold crystallization above the glass transition temperature ${ }^{34}$. On the contrary, in the case of the $X_{t}=1$ sample, no cold crystallization is observed, confirming that the maximum crystallinity was attained. In Figure $6.5(\mathrm{~b})$ the $G^{\prime}$ is plotted as a function of temperature and exactly matches the trends observed in E', Figure 6.5(a).

At room temperature, the increase in stiffness pertaining to crystallization is $21 \%$ (512 $\mathrm{MPa}$ to $620 \mathrm{MPa})$. At body temperature $\left(\sim 37^{\circ} \mathrm{C}\right)$, the increase in stiffness is more pronounced, namely $106 \%$ (243 MPa to $500 \mathrm{MPa}$ ). Retrospectively, the approach of reducing $\mathrm{T}_{\mathrm{g}}$ close to $37^{\circ} \mathrm{C}$ confirms substantial variations in stiffness with variations in crystallinity. However, it must be noted that the bulk properties are measured via DMTA and may not always represent stiffness at the surface. In the context of tissue engineering, it is important to study the surface properties as cell response relies on their adhesion to a substrate ${ }^{20-24}$. Therefore, the local surface stiffness was investigated by indentation methods. In Figure 6.5(c), the force was recorded as a function of displacement during the loading and unloading cycles. The measurements were performed at two temperatures: $23^{\circ} \mathrm{C}$ and $37^{\circ} \mathrm{C}$. 

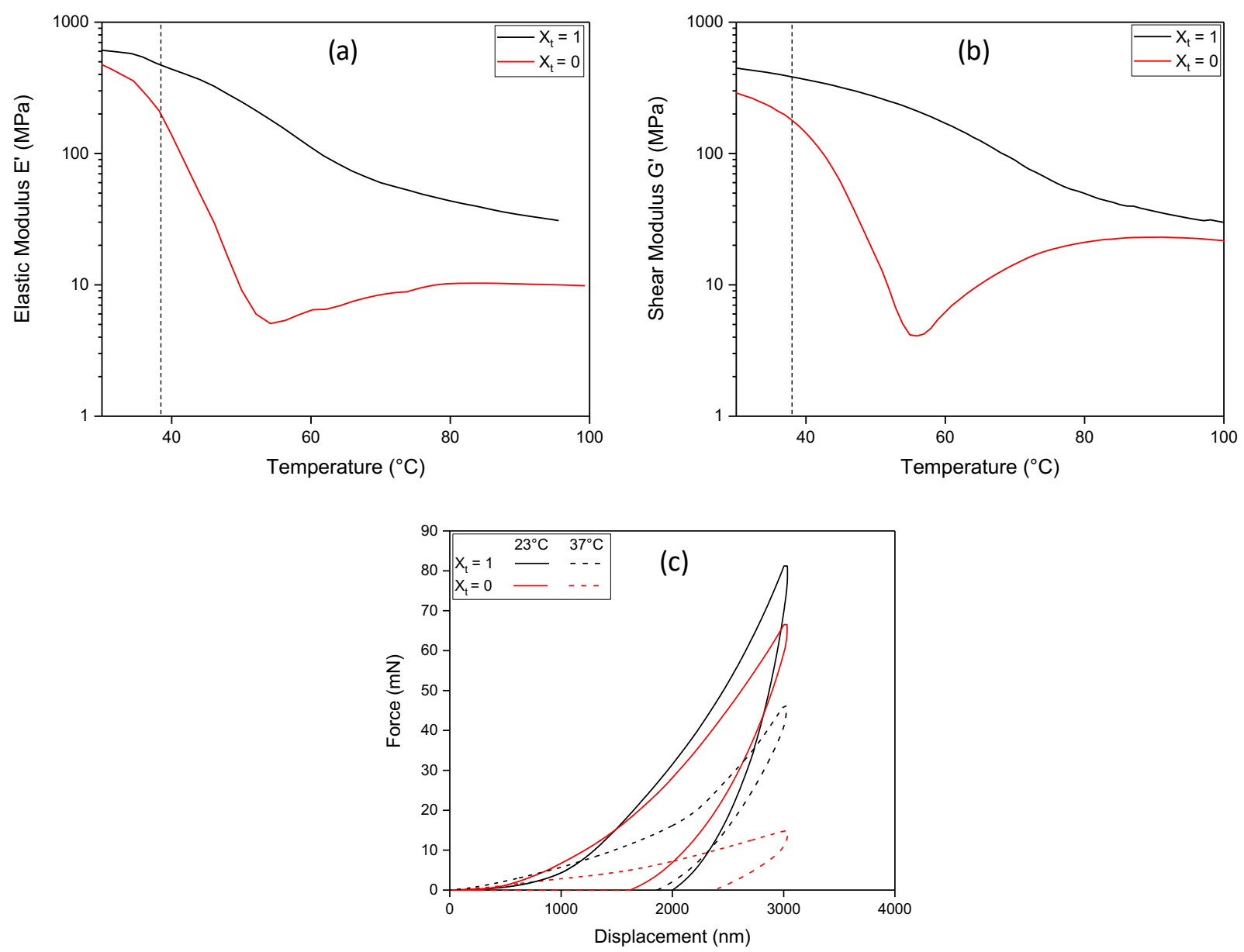

Figure 6.5: The dynamic (a) elastic modulus ( $\left.E^{\prime}\right)$ and (b) shear modulus (G') of PLLA-V18 plotted for $X_{t}$ $=0$ and $X_{t}=1$ as a function of temperature, recorded by means of DMTA; (c) Indentation derived forcedisplacement diagrams of films with $X_{t}=0$ and $X_{t}=1$ relative crystallinity to evaluate the effect of crystallinity on surface stiffness. $X_{t}=0$ represents an amorphous substrate, while $X_{t}=1$ represents $a$ substrate with maximum crystallinity. Tests were performed at $23^{\circ} \mathrm{C}$ and $37^{\circ} \mathrm{C}$ respectively.

Table 6.1: Contact stiffness(S) and maximum force $\left(F_{\max }\right)$ for samples with $X_{t}=0$ and $X_{t}=1$ recorded by indentation at $23^{\circ} \mathrm{C}$ and $37^{\circ} \mathrm{C}$.

\begin{tabular}{|c|c|c|c|}
\hline Sample & Temperature $\left({ }^{\circ} \mathrm{C}\right)$ & Contact Stiffness S $(\mathrm{N} / \mathrm{m})$ & $\mathrm{F}_{\max }(\mathrm{mN})$ \\
\hline \multirow{2}{*}{$\mathrm{X}_{\mathrm{t}=0}$} & 23 & $10 \times 10^{4}$ & 66 \\
\cline { 2 - 4 } & 37 & $1.4 \times 10^{4}$ & 13 \\
\hline \multirow{2}{*}{$X_{\mathrm{t}=1}$} & 23 & $13 \times 10^{4}$ & 82 \\
\cline { 2 - 4 } & 37 & $4.3 \times 10^{4}$ & 47 \\
\hline
\end{tabular}


From Figure 6.5(c), the maximum force during the measurement $\left(F_{\max }\right)$ and contact stiffness $(S)$ were registered and reported in Table 6.1. For the calculation of $\mathrm{S}$, the method used by Pharr et. al. was used. Here, the initial slope of the unloading curve represents $S^{35}$. The results at $23^{\circ} \mathrm{C}$ and $37^{\circ} \mathrm{C}$ reveal that both $\mathrm{S}$ and $\mathrm{F}_{\max }$ increase with crystallinity as expected. The effect is amplified at $37^{\circ} \mathrm{C}$, as seen by a $207 \%$ increase in $\mathrm{S}$ and a $261 \%$ increase in $\mathrm{F}_{\max }$. The above results confirm that (a) substantial variation in surface stiffness can be achieved for the selected material composition, and (b) the range of stiffness is within the range observed in the osteochondral interface $24,25,29,30$. In the next section, the knowledge is transferred into the making of a "gradient scaffold" via FDM.

\subsubsection{Printing gradient scaffold}

To demonstrate control in the realization of a spatial gradient in crystallinity in a porous 3D printed scaffold, i.e. under non-isothermal conditions, the thermal profile during printing was first evaluated. Based on the observations in Chapter 2, it is likely that crystallization will proceed via cold crystallization during annealing cycles ${ }^{34}$. In this way, the bottom layer experiences most annealing cycles and is expected to have the highest crystallinity. To ensure that the bottom layer attains maximum crystallinity, the printing speed was set to $50 \mathrm{~mm} / \mathrm{s}$, as it resulted in a printing time of approximately 8 minutes, comparable to the time required to reach maximum crystallinity $\left(X_{t}=1\right)$, Figure 6.4.

Furthermore, it is crucial to note that in this scenario, the heat buildup within the specimen must be avoided. The temperature profile recorded is depicted in Figure 6.6. Typically, completion of a layer is marked by the immediate deposition of the next layer (no break/pause), which is represented by the measurement " 0 -sec break". Despite the porous structure, the dissipation of heat after the deposition of each layer did not complete. In fact, the temperature minima remain above $60^{\circ} \mathrm{C}$. To manage the temperature more accurately during printing, additional time for more effective heat dissipation was provided by the introduction of breaks of 10 or $15 \mathrm{sec}$ after deposition of each layer. As observed in Figure 6.6, the incorporation of breaks reduced the peak minima to values just above the set build temperature of $60^{\circ} \mathrm{C}$, confirming effective heat dissipation. Following this strategy, it is likely that the local heat administered via the melt extrudate can be used to control spatial variations in (cold) crystallization. 


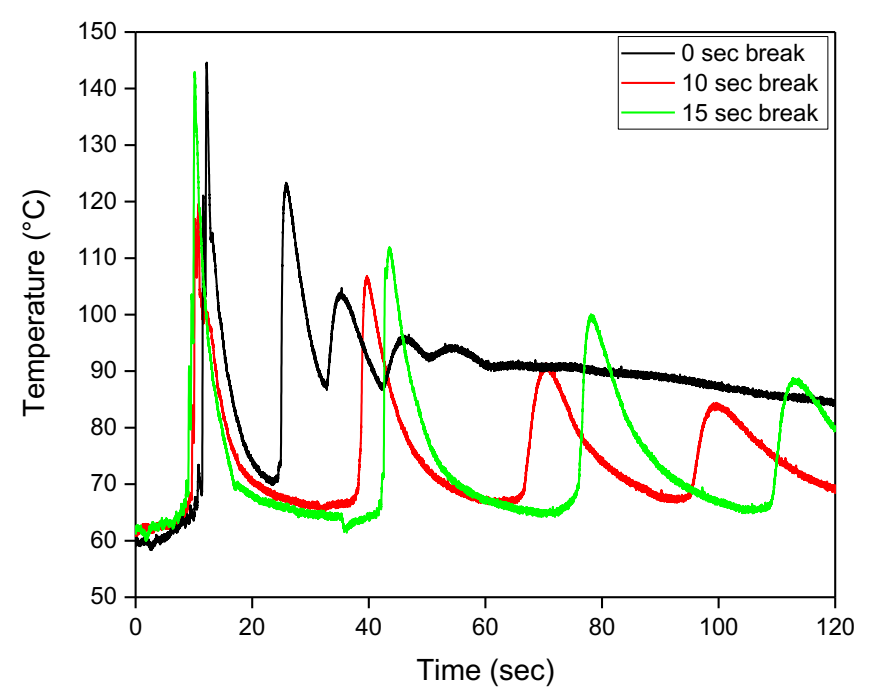

Figure 6.6: Temperature profiles of printing scaffolds from PLLA-V18 recorded at the center of the bottom layer at $50 \mathrm{~mm} / \mathrm{s}$ printing speed with breaks of 0,10 and 15 seconds after each layer deposition.

The sample prepared by employing a break of $10 \mathrm{sec}$ after the deposition of each layer was characterized in further detail. The spatial gradient in stiffness in the sample was evaluated by means of WAXD of the bottom (layer 1), middle (layer 9) and top (layer 18) layers after isolation. The 2D WAXD patterns for layers 1, 9 and 18 are shown in Figure 6.7. The intensity of the broad amorphous halo relative to the intensity of the crystal-induced sharp circumferential diffraction signals enables the calculation of layer-specific crystallinity. Based on the azimuthal integration of the 2D WAXD patterns, the crystallinity was evaluated. The results confirm that under the adopted printing protocol a clear gradient in crystallinity along the build direction exists as layers 1,9 and 18 possess a crystallinity of $52 \%, 44 \%$, and $0 \%$ respectively.

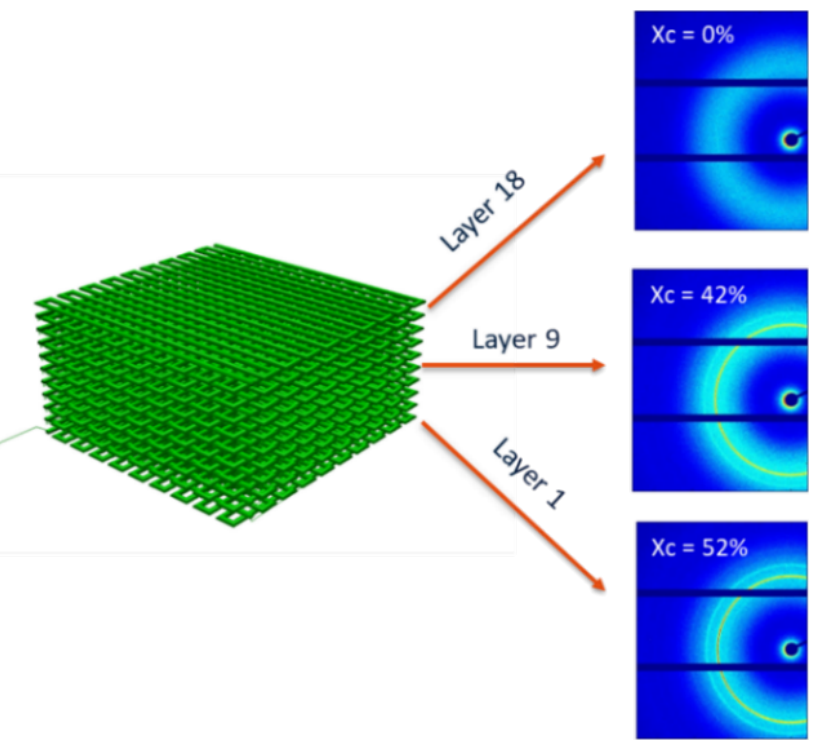

Figure 6.7: 2D WAXD patterns of layer1, 9 and 18 revealing the crystallinity gradient achieved. 


\subsubsection{Conclusions}

A novel route to introduce "continuous stiffness gradients" in a single component (and single chemistry) FDM printed scaffolds by spatial control of crystallinity is demonstrated. The method enables the design of scaffold-based regenerative strategies for the osteochondral interface by mimicking its characteristic stiffness gradient.

As a next step, the question of whether these stiffness gradients alter human mesenchymal stromal cell differentiation is to be answered. A few points need to be taken into consideration. A cautious approach is required not to misinterpret the outcome of such a study. Other parameters, like surface roughness, are reported to potentially influence stem cell differentiation as well ${ }^{23}$. Therefore, the effect of crystallinity induced surface stiffness and surface roughness on stem cell differentiation must be studied independently. 


\section{REFERENCES}

(1) Ligon, S. C.; Liska, R.; Stampfl, J.; Gurr, M.; Mülhaupt, R., Polymers for 3D Printing and Customized Additive Manufacturing. Chemical reviews. 2017, 117 (15), 10212-10290.

(2) Rodriguez, J. F.; Thomas, J. P.; Renaud, J. E., Mechanical Behavior of Acrylonitrile Butadiene Styrene (ABS) Fused Deposition Materials. Experimental Investigation. Rapid Prototyping Journal. 2001, 7 (3), 148-158.

(3) Wang, L.; Gramlich, W. M.; Gardner, D. J., Improving the Impact Strength of Poly(Lactic Acid) (PLA) in Fused Layer Modeling (FLM). Polymer. 2017, 114, 242-248.

(4) Cicala, G.; Giordano, D.; Tosto, C.; Filippone, G.; Recca, A.; Blanco, I., Polylactide (PLA) Filaments a Biobased Solution for Additive Manufacturing: Correlating Rheology and Thermomechanical Properties with Printing Quality. Materials. 2018, 11 (7).

(5) Miyamoto, T.; Inagaki, H., The Stereocomplex Formation in Poly(Methyl Methacrylate) and the Stereospecific Polymerization of Its Monomer. Polymer Journal. 1970, 1 (1), 46-54.

(6) Nakano, K.; Hashimoto, S.; Nakamura, M.; Kamada, T.; Nozaki, K., Stereocomplex of Poly(Propylene Carbonate): Synthesis of Stereogradient Poly(Propylene Carbonate) by Regio- and Enantioselective Copolymerization of Propylene Oxide with Carbon Dioxide. Angewandte Chemie International Edition. 2011, 50 (21), 4868-4871.

(7) Xie, Q.; Yu, C.; Pan, P., Crystallization in Multiphase Polymer Systems,2018,535-573, ISBN 978--012-809453-2.

(8) Longo, J. M.; Diciccio, A. M.; Coates, G. W., Poly(Propylene Succinate): A New Polymer Stereocomplex. Jounral of American Chemical Society. 2014, 136 (45), 15897-15900.

(9) Ramot, Y.; Haim-Zada, M.; Domb, A. J.; Nyska, A., Biocompatibility and Safety of PLA and Its Copolymers. Advanced Drug Delivery Reviews. 2016,153-162.

(10) Saini, P.; Arora, M.; Kumar, M. N. V. R., Poly(Lactic Acid) Blends in Biomedical Applications. Advanced Drug Delivery Reviews. 2016, 47-59.

(11) Drumright, R. E.; Gruber, P. R.; Henton, D. E., Polylactic Acid Technology. Advanced Materials. 2000, 12 (23), 1841-1846.

(12) Olszta, M. J.; Cheng, X.; Jee, S. S.; Kumar, R.; Kim, Y. Y.; Kaufman, M. J.; Douglas, E. P.; Gower, L. B. Bone Structure and Formation: A New Perspective. Materials Science and Engineering Reports. 2007, 77-116.

(13) Kuo, C. K.; Li, W.-J.; Mauck, R. L.; Tuan, R. S. Cartilage Tissue Engineering: Its Potential and Uses. Current Opinion in Rheumatology. 2006, 18 (1), 64-73.

(14) Solchaga, L.A.; Goldberg, V.M.; Caplan, A.I., Cartilage Regeneration Using Principles of Tissue Engineering. Clinical Orthopaedics and Related Researches of Tissue. 2001, 391, 161-170

(15) Bible, T.; McKinley, M.; O'Loughlin, V., Anatomy \& Physiology: An Integrative Approach; McGrawHill Education, 2015, ISBN 9780078024283.

(16) Breedveld, F. C. Osteoarthritis-the Impact of a Serious Disease. Rheumatology 2004, 43, i4-i8.

(17) Kurtz, S.; Ong, K.; Lau, E.; Mowat, F.; Halpern, M., Projections of Primary and Revision Hip and Knee Arthroplasty in the United States from 2005 to 2030. Journal of Bone Joint. Surgery. 2007, 89 (4), 780-785.

(18) Blagojevic, M.; Jinks, C.; Jeffery, A.; Jordan, K. P., Risk Factors for Onset of Osteoarthritis of the Knee in Older Adults: A Systematic Review and Meta-Analysis. Osteoarthritis and Cartilage. 2010, 18 (1), 24-33.

(19) Bose, S.; Roy, M.; Bandyopadhyay, A., Recent Advances in Bone Tissue Engineering Scaffolds. Trends in Biotechnology. 2012, 546-554.

(20) Ferris, C. J.; Gilmore, K. G.; Wallace, G. G.; in het Panhuis, M., Biofabrication: An Overview of the Approaches Used for Printing of Living Cells. Applied Microbiology and Biotechnology. 2013, 97 (10), 4243-4258.

(21) Murphy, W. L.; Dennis, R. G.; Mooney, D. J., Tissue Engineering Scaffolds. United States Patent US7575759B2, 2009.

(22) Rush, M. N.; Coombs, K. E.; Hedberg-Dirk, E. L., Surface Chemistry Regulates Valvular Interstitial Cell Differentiation in Vitro. Acta Biomateriala. 2015, 28, 76-85.

(23) Faia-Torres, A. B.; Charnley, M.; Goren, T.; Guimond-Lischer, S.; Rottmar, M.; Maniura-Weber, K.; Spencer, N. D.; Reis, R. L.; Textor, M.; Neves, N. M., Osteogenic Differentiation of Human Mesenchymal Stem Cells in the Absence of Osteogenic Supplements: A Surface-Roughness Gradient Study. Acta Biomaterialia. 2015, 28, 64-75.

(24) Di Luca, A.; Van Blitterswijk, C.; Moroni, L., The Osteochondral Interface as a Gradient Tissue: From Development to the Fabrication of Gradient Scaffolds for Regenerative Medicine. Birth 
Defects Research. Part C: Embryo Today Reviews. 2015, 105 (1), 34-52.

(25) Di Luca, A.; Longoni, A.; Criscenti, G.; Mota, C.; van Blitterswijk, C.; Moroni, L., Toward Mimicking the Bone Structure: Design of Novel Hierarchical Scaffolds with a Tailored Radial Porosity Gradient. Biofabrication. 2016, 8 (4), 045007.

(26) Guilak, F.; Cohen, D. M.; Estes, B. T.; Gimble, J. M.; Liedtke, W.; Chen, C. S., Control of Stem Cell Fate by Physical Interactions with the Extracellular Matrix. Cell Stem Cell. 2009, 17-26.

(27) Di Luca, A.; Longoni, A.; Criscenti, G.;Lorenzo-Moldero, I.; Klein-Gunnewiek, M.; Vancso, J.; van Blitterswijk, C.; Mota, C.; Moroni, L., Surface energy and stiffness discrete gradients in additive manufactured scaffolds for osteochondral regeneration. Biofabrication. 2016, 8

(28) Ma, Z.; Kotaki, M.; Inai, R.; Ramakrishna, S., Potential of Nanofiber Matrix as Tissue-Engineering Scaffolds. Tissue Engineering. 2005, 11 (1-2), 101-109.

(29) Langer, R.; Vacanti, J. P., Tissue Engineering. Science. 1993, 260 (5110), 920-926.

(30) Athanasiou, K. A.; Zhu, C.-F.; Lanctot, D. R.; Agrawal, C. M.; Wang, X., Fundamentals of Biomechanics in Tissue Engineering of Bone. Tissue Engineering.. 2000, 6 (4), 361-381.

(31) Brigelius-Flohé, R., Bioactivity of Vitamin E. Nutrition Research Reviews. 2006, 174-186.

(32) Piroddi, M.; Pilolli, F.; Aritomi, M.; Galli, F., Vitamin E as a Functional and Biocompatibility Modifier of Synthetic Hemodialyzer Membranes: An Overview of the Literature on Vitamin EModified Hemodialyzer Membranes. American Journal of Nephrology. 2012, 35 (6), 559-572.

(33) Dutta-Roy, A. K.; Gordon, M. J.; Campbell, F. M.; Duthie, G. G.; James, W. P. T., Vitamin E Requirements, Transport, and Metabolism: Role of a-Tocopherol-Binding Proteins. The Journal of Nutritional Biochemistry. 1994, 562-570.

(34) Srinivas, V.; van Hooy-Corstjens, C. S. J.; Harings, J. A. W., Correlating Molecular and Crystallization Dynamics to Macroscopic Fusion and Thermodynamic Stability in Fused Deposition Modeling; a Model Study on Polylactides. Polymer. 2018, 142, 348-355.

(35) Pharr, G. M.; Oliver, W. C., Measurement of Thin Film Mechanical Properties Using Nanoindentation. MRS Bulletin. 1992, 17 (7), 28-33. 


\section{Appendix A}

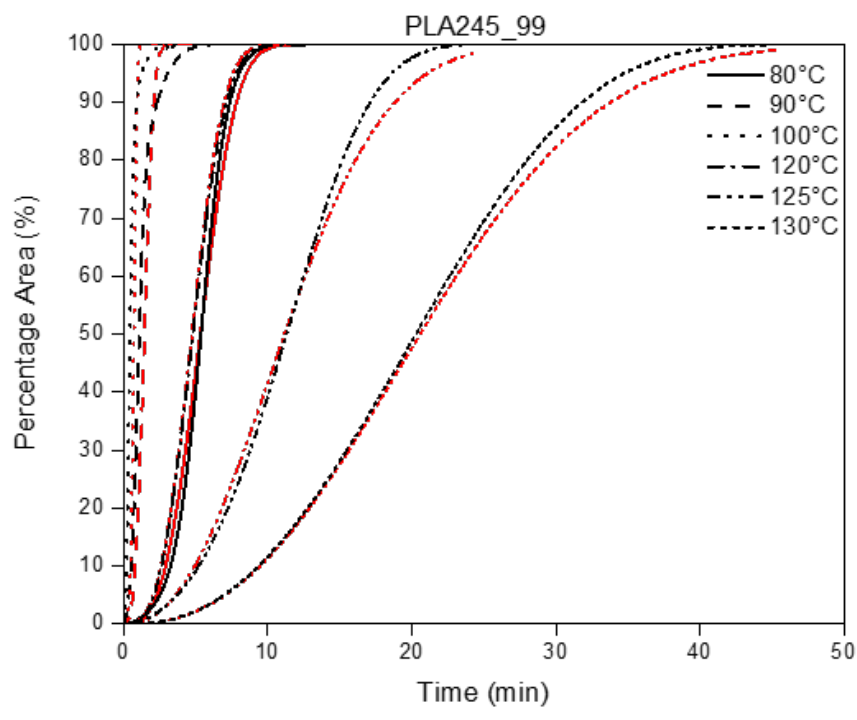

(a)

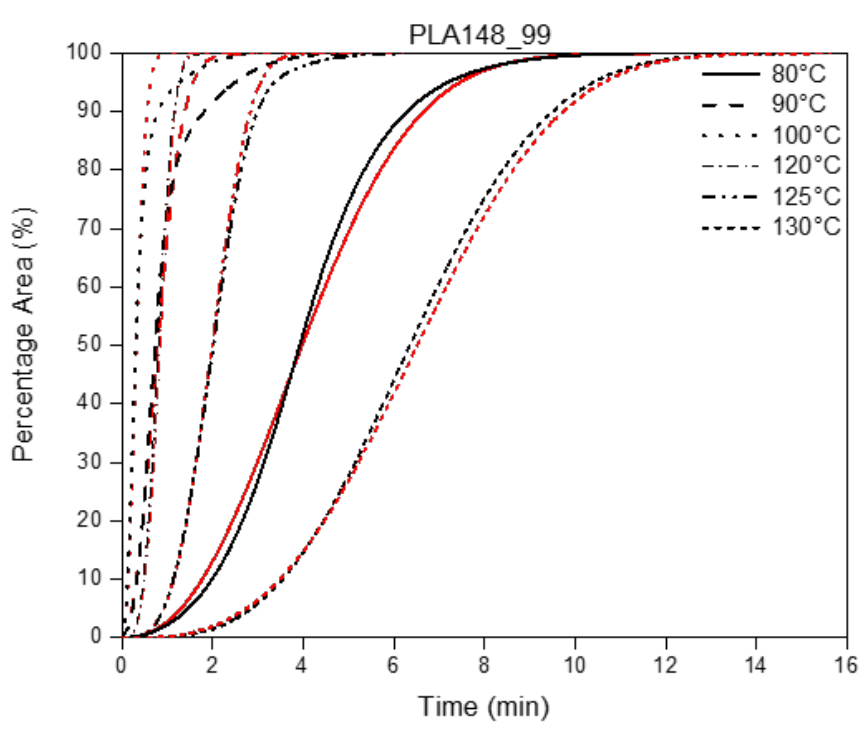

(b)

Figure A1: Experimental data (in black) and corresponding Avrami fits (in red) for isothermal crystallization of (a) PLA245_99 and (b) PLA148_99. Note that the fits are representative of the primary crystallization process. After the crystallization half-time deviations of the Avrami fits become apparent and are caused by secondary crystallization effects.

Table A1: Void fraction calculated with and without perimeter.

\begin{tabular}{|c|c|c|c|}
\hline Sample & $\begin{array}{c}\text { Speed } \\
{[\mathrm{mm} / \mathrm{s}]}\end{array}$ & $\begin{array}{c}\text { Void fraction } \\
\text { with perimeter } \\
{[\%]}\end{array}$ & $\begin{array}{c}\text { Void fraction } \\
\text { without } \\
\text { perimeter [\%] }\end{array}$ \\
\hline \multirow{2}{*}{ PLA245_99 } & 20 & 2.82 & 0.31 \\
\cline { 2 - 4 } & 50 & 1.40 & 0.48 \\
\hline \multirow{2}{*}{ PLA245_96 } & 20 & 1.20 & 0.32 \\
\cline { 2 - 4 } & 50 & 4.82 & 0.49 \\
\hline \multirow{2}{*}{ PLA148_99 } & 20 & 3.86 & 0.15 \\
\cline { 2 - 4 } & 50 & 1.50 & 0.16 \\
\hline
\end{tabular}




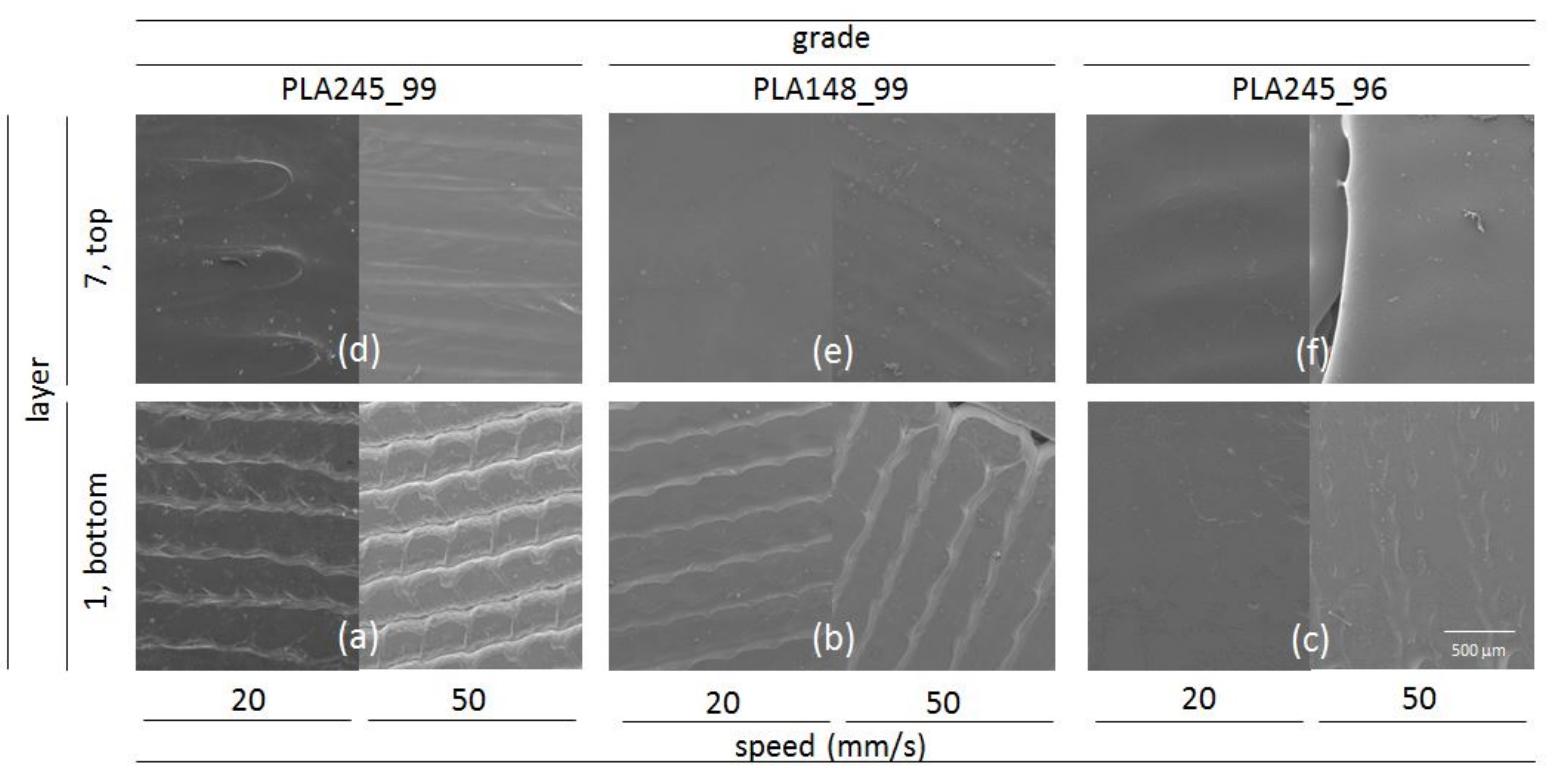

Figure A2: Scanning electron micrographs of the xy top (upper) and xy bottom (down) surfaces of for PLA245_99 (a, d), PLA_148_99 $(b, e)$ and PLA245_96 (c, f) visualizing the effect of molar mass, Lenantiomeric purity and print speed, which is $20 \mathrm{~mm} / \mathrm{s}$ for the left and $50 \mathrm{~mm} / \mathrm{s}$ for the right images. The scale bar is representative to all images.

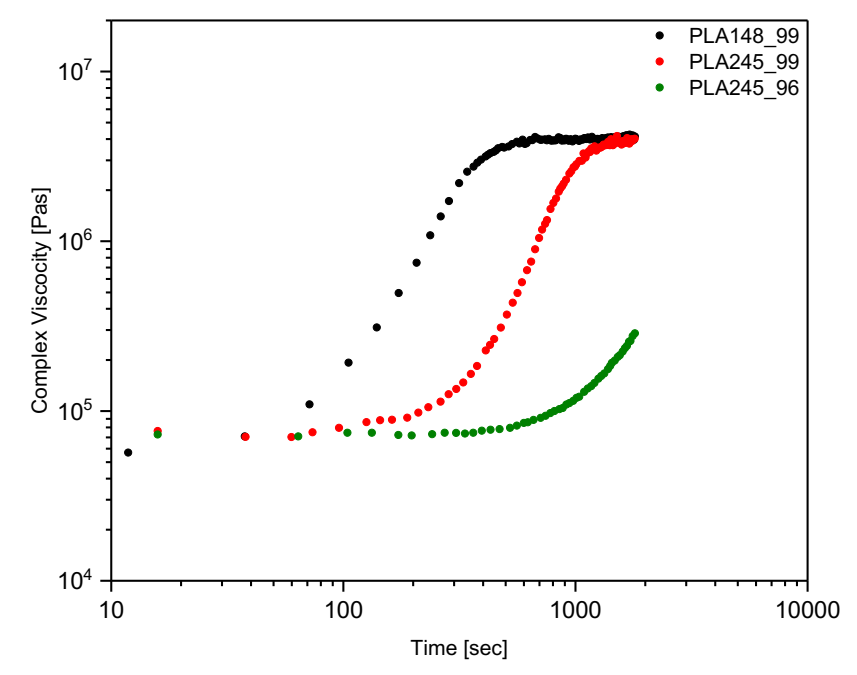

Figure A3: Influence of crystallization, isothermal at $120{ }^{\circ} \mathrm{C}$, on complex viscosity of poly(lactides) (PLA148_99, PLA245_99 and PLA245_96). 


\section{Appendix B}

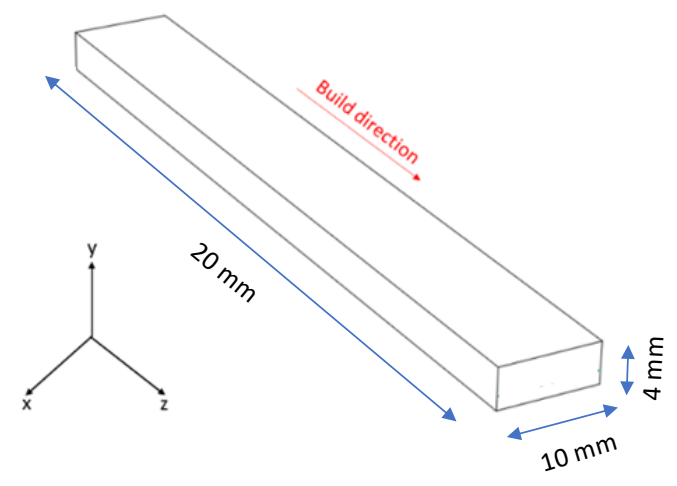

Figure B1: Schematic of sample geometry.

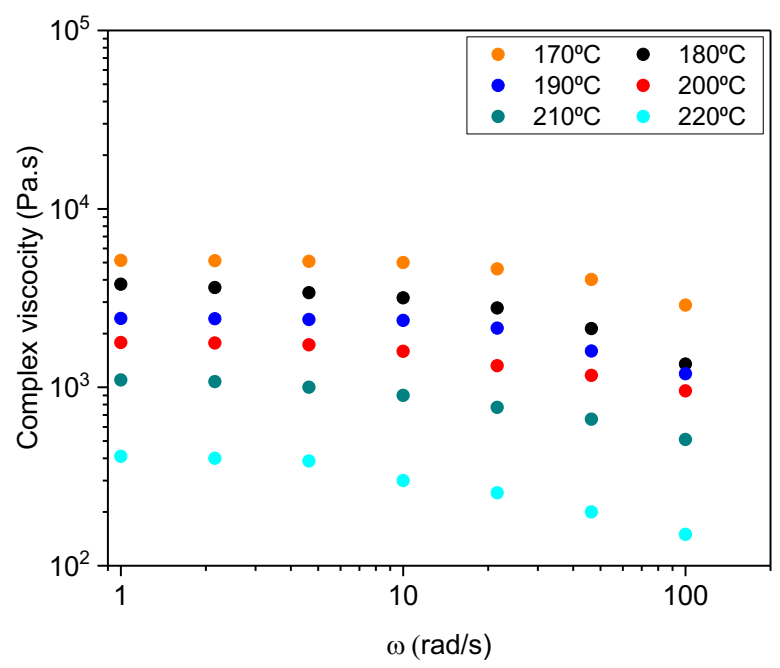

Figure B2: Complex viscosity plotted as a function of angular frequency ( $\omega)$ for L245 at different temperatures to determine printability window. 

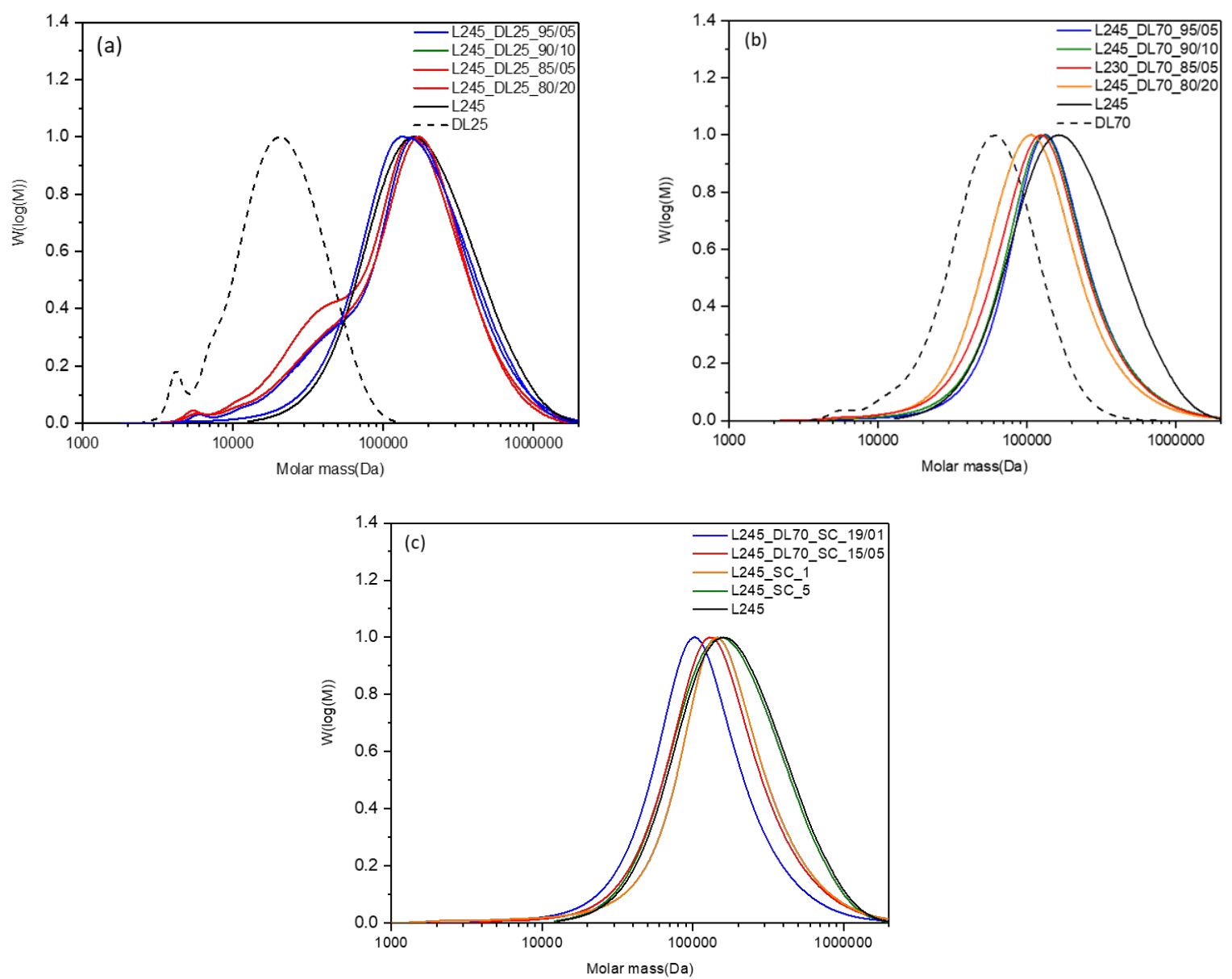

Figure B3: Molecular weight distributions of (a) L245-DL25, (b) L245-DL70, (c) L245-DL70-SC and L245SC blends. 

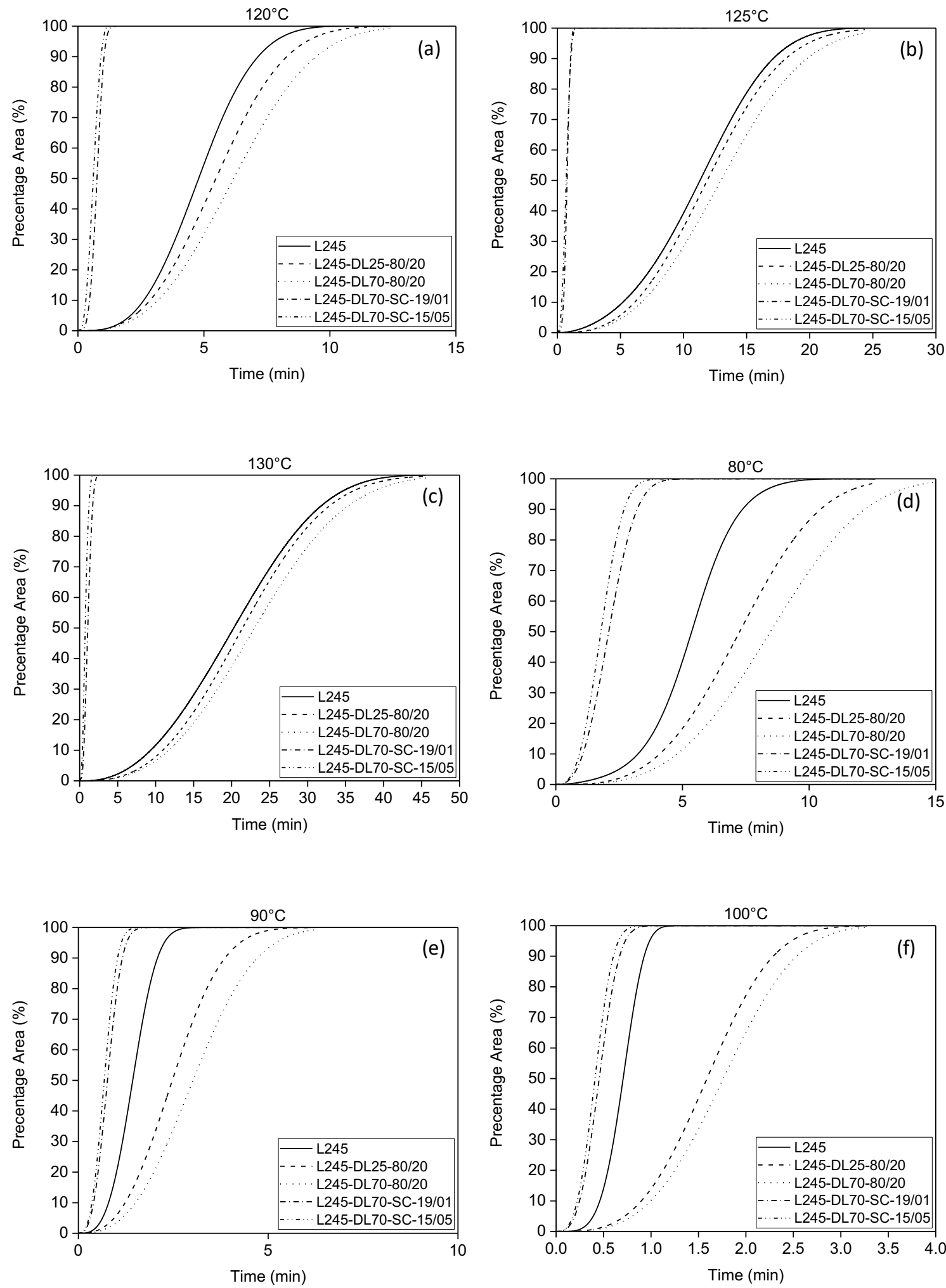

Figure B4: Development of relative crystallinity (represented by Percentage Area) as a function of time for different crystallization temperatures for L245, L245-DL25-80/20, L245-DL70-80/20, L245-DL70-SC19/01 and L245-DL70-SC-15/05. (a), (b) and (c) represent melt crystallization and (d), (e) and (f) represent cold crystallization. 

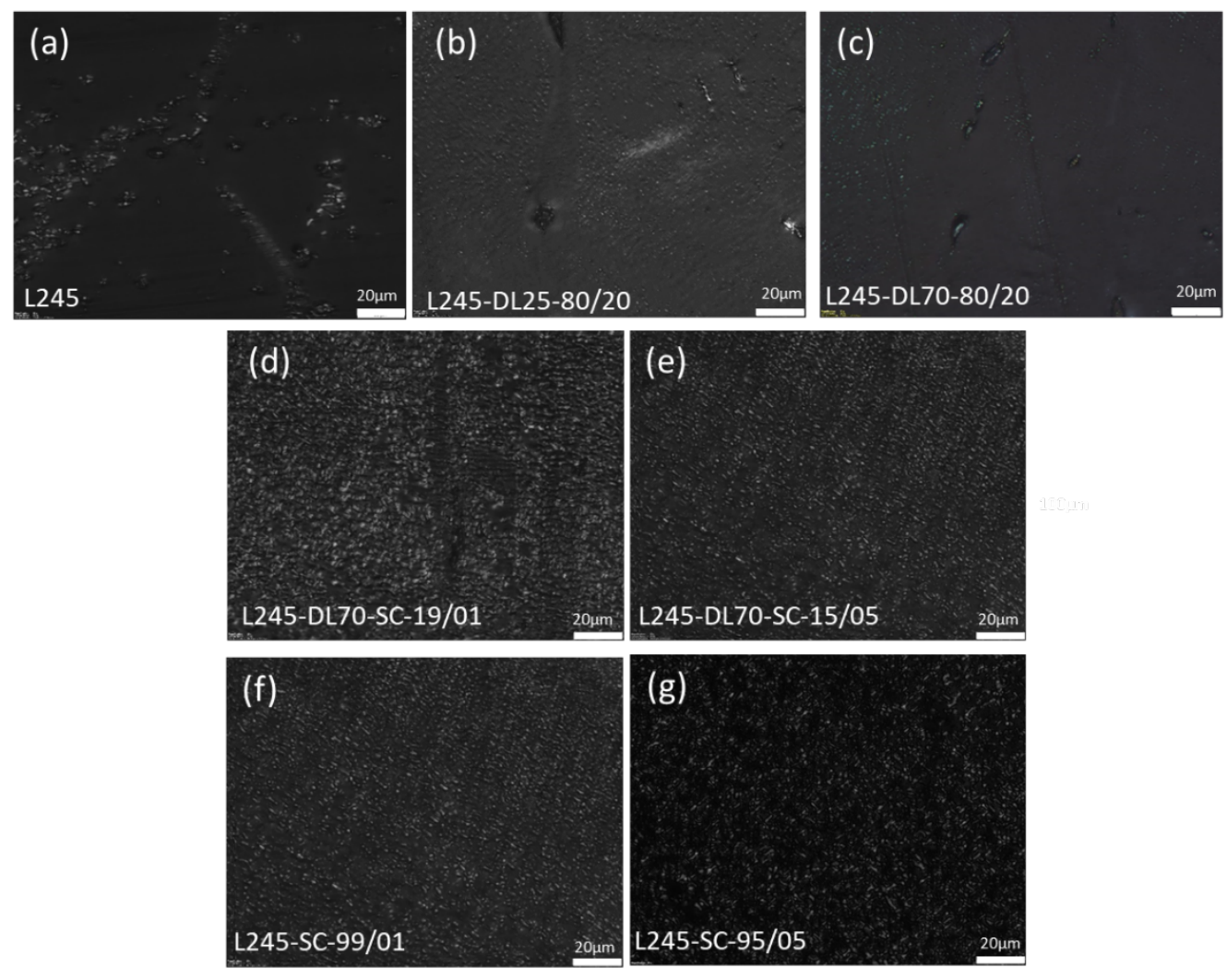

Figure B5: Polarized optical micrographs of printed samples for (a) L245, (b) L245-DL25-80/20, (c) L245DL70-80/20, (d) L245-DL70-SC-19/01, (e) L245-DL70-SC-15/05, (f) L245-SC-99/01 and (g) L245-SC-95/05 depicting the crystalline morphology.

Table B1: Glass transition $\left(T_{g}\right)$, crystallization temperature $\left(T_{c}\right)$ and crystallization enthalpy $\left(\Delta H_{m c}\right)$ for blends studied.

\begin{tabular}{|c|c|c|c|}
\hline Blend & $\mathbf{T}_{\mathbf{g}}\left({ }^{\mathbf{0}} \mathbf{C}\right)$ & $\mathbf{T}_{\mathbf{c}}\left({ }^{\mathbf{0}} \mathbf{C}\right)$ & $\Delta \mathbf{H}_{\mathbf{c m}}(\mathbf{J} / \mathbf{g})$ \\
\hline L245 & & & \\
\hline L245-DL25-80/20 & 64 & 97 & 17 \\
\hline L245-DL25-85/15 & 58 & 88 & 6.25 \\
\hline L245-DL25-90/10 & 59 & 93 & 7.64 \\
\hline L245-DL25-95/05 & 62 & 96 & 16.5 \\
\hline L245-DL70-80/20 & 56.5 & 86.5 & 16.7 \\
\hline L245-DL70-85/15 & 58 & 89 & 7 \\
\hline L245-DL70-90/10 & 60 & 92.5 & 16.2 \\
\hline L245-DL70-95/05 & 62 & 95.5 & 16.5 \\
\hline L245-DL70-SC-19/01 & 59 & 123 & 50 \\
\hline L245-DL70-SC-15/05 & 60 & 129 & 52.5 \\
\hline L245-SC-99/01 & 64 & 17 & 46 \\
\hline L245-SC-95/05 & 64 & 125 & 48 \\
\hline
\end{tabular}




\section{Appendix C}

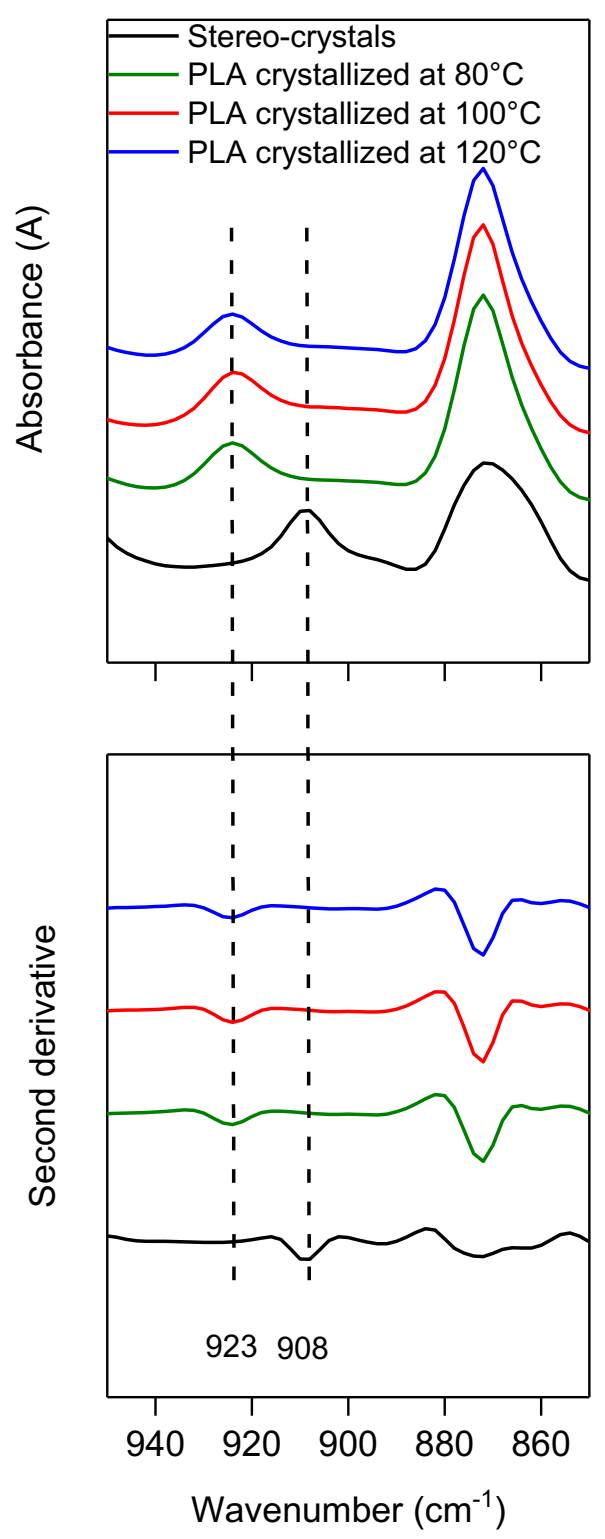

Figure C1: FTIR spectra and corresponding second derivative for PLLA148 and PDLA100 crystallized at $80^{\circ} \mathrm{C}, 100^{\circ} \mathrm{C}, 120^{\circ} \mathrm{C}$ and pure stereocrystals. Samples crystallized at $80^{\circ} \mathrm{C}, 100^{\circ} \mathrm{C}, 120^{\circ} \mathrm{C}$ represent homocrystals and were produced by keeping a fully amorphous sample at the respective annealing temperature for 1 hour. On the other hand, stereocrystals were produced by keeping a 50:50 blend of PLLA148 and PDLA100 at $200^{\circ} \mathrm{C}$ for 15 minutes, after which the sample was quenched. 


\section{Appendix D}

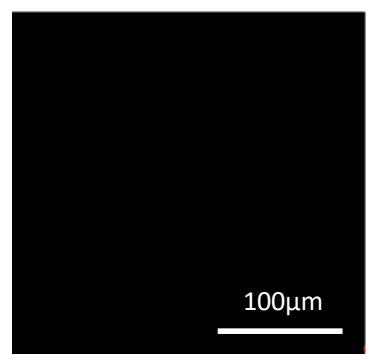

$0 \min$

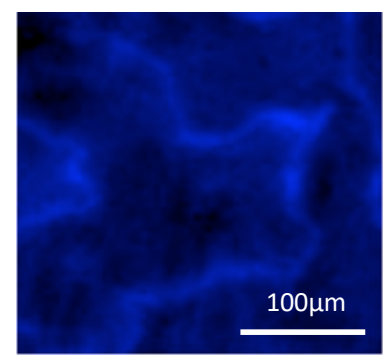

$10 \mathrm{~min}$

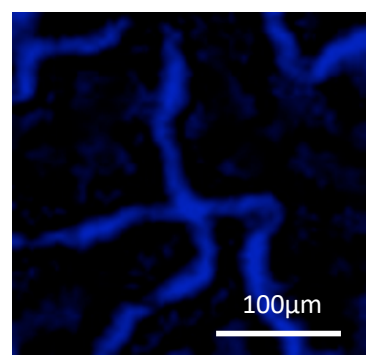

$20 \min$

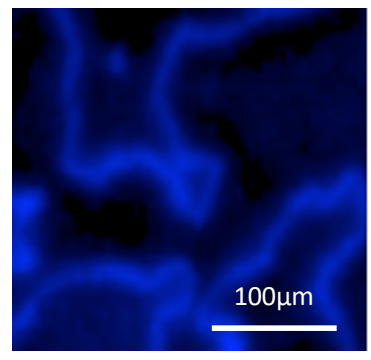

$60 \min$

Figure D1: FTIR absorbance images highlighting the structural composition of the interfaces produced for samples of L140-D90 crystallized at $200^{\circ} \mathrm{C}$ for 0, 10, 20 and 30 minutes respectively. The blue regions highlight the areas where interfacial stereocrystals are formed. The black regions highlight the regions dominated by homocrystals.

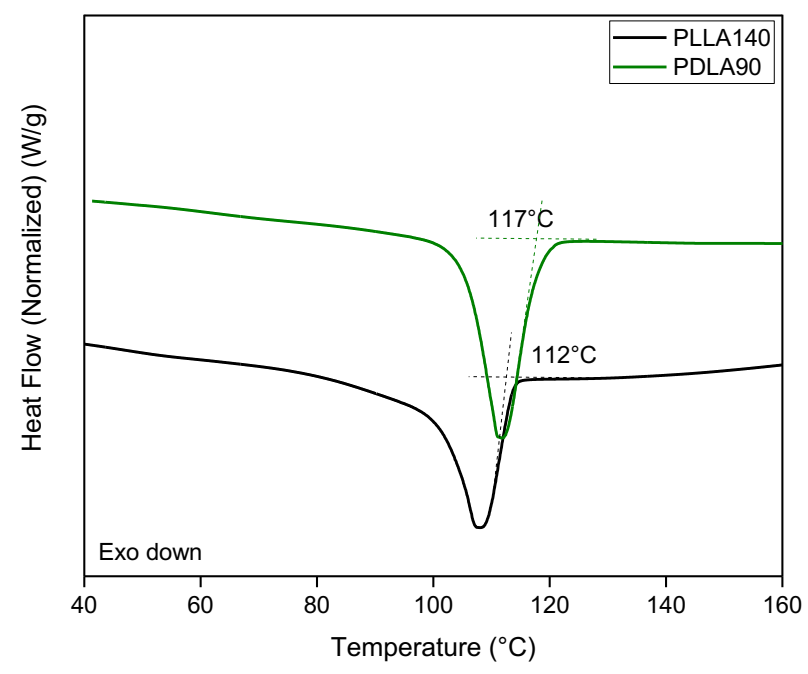

Figure D2: DSC thermograms of PLLA140 (black) and PDLA90 (green) on cooling from $200^{\circ} \mathrm{C}$ at a rate of $10^{\circ} \mathrm{C} / \mathrm{min}$, revealing the onset temperature of melt crystallization. 


\section{Acknowledgements}

My 4 years of doctoral research has been extremely fulfilling at a personal and professional level. I would like to express my gratitude to everybody who directly or indirectly contributed to this thesis. Firstly, I would like to thank Prof Sanjay Rastogi. Thanks Sanjay for allowing me to be a part of your group, your support and guidance. I wish you the best in your future endeavours. Secondly, a big thanks to my supervisors Dr. Jules Harings and Dr. Tosca van Hooy. Jules, you have been the driver of my research. This work would not have been possible without you. Thank you for being there always for my questions (even at odd hours), motivating me to always try something new and giving the freedom to conduct and shape my research. I thoroughly enjoyed our discussions and experiments. That is something I will definitely miss. Tosca, thanks for your guidance and support, I appreciate that you have always critically evaluated my results and provided a fresh perspective.

I would like to thank the members of my assessment committee, Prof. Ron Heeren, Prof Sybrand van der Zwaag, Dr. Katrien Bernerts and Prof Richard Graham for taking the time to evaluate my thesis. Sybrand, my journey in the field of polymers began in your group in TU Delft. You were inspirational in me choosing this field of study. I am glad you were my supervisor for my master thesis and agreed to be a part of my assessment committee as well.

In the past 4 years many people outside Maastricht University have helped me. I thank Gino van Strydonck and Marliene Bos from Zuyd Hogeschool for their support these four years. A major part of my lab work was conducted at CHILL and I thank everyone there for their help. Special thanks to William Warnier for helping me on numerous occasions with the Arburg printer. I would also like to thank Stan Looijmans from TU Eindhoven for the time you put in to help me with the indentation studies. I also thank Bas van Leeuwen from Corbion for actively supporting my research by providing materials whenever I needed and also being actively involved in technical aspects of this research. Your inputs have been very valuable.

I thank the first group of PhDs at AMIBM: Geert, Goose, Andrea, Manta, Ola, Marie, Marteen, Vahid, Christian, Monika and Anna at AMIBM for the nice time at Chemelot. I also thank all the colleagues at Centercourt for creating a friendly and stimulating working atmosphere. Thanks, Dario, Karel, Lawrence, Lucienne, Milo, Renato, Ramiro, Mohan, Mitch, Pouya, Feiji, Huaquin, Roel, Jurie. A special thanks to Nils for the technical help. Thanks for introducing me to most of the analysis techniques in the lab and more importantly, the continuous support during these 4 years. It was a good time both in the lab and outside! I thank all others at AMIBM, it was a pleasure to work with you and see the institute grow and develop. 
In these four years, I have also got the chance to work and supervise a number of students. They have contributed significantly to this research. Thanks Francesca, Linda, Gies, Indy, Sophie, Laurent and Demy for the good work you have done. This thesis would be incomplete without your efforts. It was a pleasure to work with you all. I would like to make a special mention of Francesca, who happens to be the only Master student on the list. Francesca, in a short time you managed to integrate and do a lot of lab work. I am sure you had a good time in Maastricht and were happy with the outcome of your work.

At the end of my thesis, I began a new chapter in my career, by joining Wavin Technology and Innovation. These past months have been busy, where I had to move to a new city, start a new job and wrap up my thesis. I appreciate the support of my colleagues at Wavin during this time and helping me settle in my new role.

In Maastricht, I have made some good friends. They will always remain dear to me. Tennis was a big part of my routine, and I thank the tennis buddies I made. Especially, I must mention Clara. I thoroughly enjoyed our countless tennis battles and the time we have spent outside the court. Namrata, I enjoyed the numerous cooking sessions we had and all the enjoyable times. Geert, Andrea and Goose, us landing up in one office was the best thing that happened during my $\mathrm{PhD}$. We became from colleagues to very close friends. You all have been like my family in the Netherlands. Andrea, you are one of the most entertaining personalities I've encountered. Life in the office and outside would certainly be less glamerouuuus (sorry can't resist) without you. Goose, thanks for the countless polymer related discussions and coffee breaks. Geert, it was amazing to share Casa Vagabond with you. Our apartment was the social hub in Maastricht and we have seen many good times there. The four of us have had many fun moments; the list is long. I fondly remember the times we have had together and for sure many more will come! Manta, what can I say about you? We started a bit slow, but we became very good friends. You are the nicest person I have met. Thanks for always being there when I needed, you are one of my most trusted friends. I wish you and Anand all the happiness. We will meet soon!

Lastly, I thank my family. I thank my grandparents for the love and support they have always given me. The biggest thanks are reserved for my parents. Mom and dad, this thesis is dedicated to you. Whatever I have achieved is because of you both. Thank you for always supporting my dreams, advising me and believing in me. 


\section{Curriculum Vitae}

Varun Srinivas was born in Bangalore (India) on 25 th May 1991. In 2013 Varun obtained his bachelor's degree in mechanical engineering from Manipal University, India. As part of bachelor thesis, he worked with Honeywell Technology Solutions, Bangalore. His work focused on simulating the rotor dynamic stability of turbochargers. During his bachelor's he also undertook several projects on manufacturing and testing of fiber-reinforced polymer composites. In the fall of 2013, Varun started his Master's at

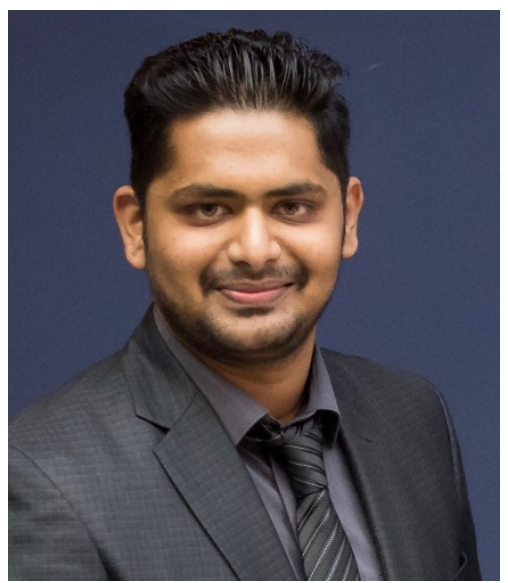
TU Delft (The Netherlands) in the field of Aerospace Engineering, in the group of Novel Aerospace Materials. He performed his Master internship and thesis at DSM Materials Research Center in Chemelot, Geleen (The Netherlands). His research focused on studying the creep and drawability of ultrahigh molecular weight polyethylene fibers on variations in molecular architecture. After completion of his Masters, he pursued his doctoral studies at Maastricht University, under the supervision of Dr. Prof Sanjay Rastogi, Dr. Jules Harings and Dr. Tosca van Hooy. The important results of his PhD research are summarized in this manuscript. During his $\mathrm{PhD}$ he also completed courses in polymer properties, polymer chemistry and polymer physics organized under the RPK program by Dutch national postgraduate research school (Polymer Technology Netherlands). In November 2019 he started a new phase in his professional career by joining Wavin Technology and Innovation in the role of Material Expert. 


\section{List of publications}

Publications that have contributed to this thesis:

Srinivas, V.; van Hooy-Corstjens, C. S. J.; Harings, J. A. W., Correlating Molecular and Crystallization Dynamics to Macroscopic Fusion and Thermodynamic Stability in Fused Deposition Modeling; a Model Study on Polylactides. Polymer. 2018,142, 348-355.

Srinivas, V.; van Hooy-Corstjens, C. S. J.; Rastogi, S.; Harings, J. A. W., Promotion of molecular diffusion and/or crystallization in fused deposition modeled poly(lactide) welds. Polymer. 2020,202, 348-355, 122637.

Srinivas, V.; van Hooy-Corstjens, C. S. J.; Vaughan, G. B. M.; van Leeuwen, B.; Rastogi, S.; Harings, J. A. W. Interfacial Stereocomplexation To Strengthen Fused Deposition Modeled Poly(Lactide) Welds. ACS Applied Polymer Materials. 2019, 1 (8), 2131-2139.

Other publications by author (not included in this thesis):

de Kort, G.W.; Saidi S.; Hermida-Merino D.; Leoné N.; Srinivas V.; Rastogi S.; Carolus H. R. M. Wilsens. Importance of Viscosity Control for Recyclable Reinforced Thermoplastic Composites. Macromolecules. 202053 (15), 6690-6702. 